TROPICAL SANDSTONE DEGRADATION WITH TIME:

CASE STUDY IN KOTA KINABALU

SABAH, MALAYSIA

Frederick Francis Tating 


\section{Examining committee:}

Prof.dr. F.D. van der Meer University of Twente / ITC

Prof.dr.ir. A. Veldkamp

Prof.dr. L.R. e Sousa

University of Twente / ITC

Prof.dr. S.R. Hencher

University of Porto

University of Leeds

Dr.ir. M. Huisman

Heerema Marine Contractors

ITC dissertation number 276

ITC, P.O. Box 217, 7500 AE Enschede, The Netherlands

ISBN 978-90-365-3951-7

DOI $10.3990 / 1.9789036539517$

Cover designed by Job Duim

Printed by ITC Printing Department

Copyright (c) 2015 by F.F. Tating

17C FACULTY OF GEO-INFORMATION SCIENCE AND EARTH OBSERVATION 


\title{
TROPICAL SANDSTONE DEGRADATION WITH TIME: CASE STUDY IN KOTA KINABALU \\ SABAH, MALAYSIA
}

\author{
DISSERTATION
}

to obtain

the degree of doctor at the University of Twente, on the authority of the rector magnificus, prof.dr. H. Brinksma, on account of the decision of the graduation committee, to be publicly defended on Wednesday 2 September 2015 at 12.45 hrs

by

Frederick Francis Tating

born on February 7, 1969

in Tambunan Sabah, Malaysia 
This thesis is approved by

Prof. dr. V.G. .Jetten, promoter

Dr. H.R.G.K. Hack, co-promoter 


\section{Acknowledgements}

Frankly, this thesis could not have been completed without the great support, help, and encouragement from many wonderful people; therefore, I would like to express my sincere thanks and appreciation for all of them.

First of all, I express my sincere gratitude to the Minerals and Geoscience Department of Malaysia for granting me the opportunity to pursue the PhD study at ITC, Enschede, The Netherlands. The support is very much appreciated of the Director General of the Minerals and Geoscience Department, Malaysia, Dato' Yunus Abdul Razak, and the Director of the Minerals and Geoscience Department, Malaysia, Kota Kinabalu, Sabah, Mr. Alexander Yan. The support of several other staff in the Minerals and Geoscience Department, Malaysia, Kota Kinabalu with whom I worked during the tenure of the research is also very much appreciated. I also express my appreciation to the Malaysian Government under the In-service Training Scheme, Human Capital Development Division of the Public Service Department of Malaysia, for financially supporting the study and living expenses in the Netherland.

My heartfelt gratitude goes to my promotor, Prof. Dr. Victor G. Jetten and copromotor/supervisor Dr. Robert Hack for their patience and untiring guidance which they bestowed on me from the earlier stages to the successful completion of the research work. Prof. Dr. Victor Jetten has always been supportive and persistently proposed ideas to enable the continuity of the research. Thank you Victor, for the zeal and enthusiasm, which inspired me. Dr. Robert Hack played an important role in shaping the course for the research. His vast experience and knowledge in the field of the research coupled with very valuable and constructive comments, suggestions, and encouragements during our discussions resulted in the main objective of the research in the present form. His untiringly commitment and supervision at every step of the research, critical comments, and reviewing the resulting articles prior to publication are very much appreciated. Thank you very much Robert, for your commitment and patience in editing the thesis.

Special thanks go to the lecturers of Earth System Department (ESA) at ITC for their dedication in providing me with valuable knowledge and skills on research techniques. Thank you Dr. David G. Rossiter, Dr. Cees van Westen, Dr. Mark van der Meijde, Dr. Tsehaie Woldai, Dr. Dhruba P. Shrestha, Drs. Robert Voskuil, Drs. Nanette Kingma, Mr. Wim Bakker, and Drs. Michiel Damen. Special thanks also go to Drs. Boudewijn de Smeth for his assistant in chemical analysis of rock samples.

I am also very grateful to all the people at ITC - past and present; and the management team, especially Paul van Dijk, Loes Colenbrander, Theresa van 
den Boogaard, Bettine Geerdink, Marie Chantal Metz, Marion Pierik, Job Duim, Benno Masselink and Saskia Groenendijk. The staff members of the ITC Hotel, ITC library, and help desk are acknowledged for their great support during my stay at ITC, Enschede. Special appreciation is given to Loes Colenbrander for her help in making this thesis ready for printing and all the necessary arrangements related to it. Ibu Dewi Pikaar is accredited for her hospitality during our stay in Enschede especially to my family members and for the delicious Indonesian food.

I extend a special appreciation to Rev. Fr. Ben Engelbertink who served several years as a missionary in Sarawak, Malaysia for his spiritual support during our stay in Enschede. Fr. Ben also arranged the visit to the Mill Hill retirement house in Oosterbeek, Netherlands to meet with Rev. Fr. Anthony Putman (now deceased), which is one of the missionary priest responsible for the education development in my hometown (Tambunan, Sabah) in the 70's. Kotoluadan Paado.

To my Malaysian friends in Enschede, Khamarul, Fauzi, Hakim and their family - thanks for the sweet moments we experienced and shared together in Enschede and hoping that our mutual friendship will continuously cherish in the future. My colleagues in the Department of Earth System Analysis of whom most of them have completed their research by now, were always a great source of technical advice. The discussions with them provided a lot of insight into my research. Just to name some, Nugroho, Sharif, Ajay, Byron, Pablo, Tolga, Shaffique, Xuanmei, Sekhar, Sharon, Anandita, Fekerte, Sumbal Saba, Sanaz, and Nasrullah, I wish all of you very good luck in the future.

Special appreciation and thanks goes to my sweet wife, Margritta Surang, and beloved son, Carl Taro, for their patience, encouragement, motivation, and support, which kept me going throughout the research. Special appreciation also goes to my younger brother, Gilbert, for accompanying me in the field, which is very much appreciated. I am not forgetting my family members and in-law family members for their constant support, encouragements, and spiritual support for the success of the study and for our safety.

Throughout the research period, I came through some difficult times. I lost both my parents; my father past away in the early stage of the research (November 2009), whereas my mother passed away just before I finished this thesis (October 2013). It is very unfortunate that we are not be able to share our joy with them. Therefore, I wish to dedicate this work to them.

Finally, I wish to express my profound gratitude and thanksgiving to the almighty God for his guidance, protection, and love over the years for my family and me. 


\section{Table of Contents}

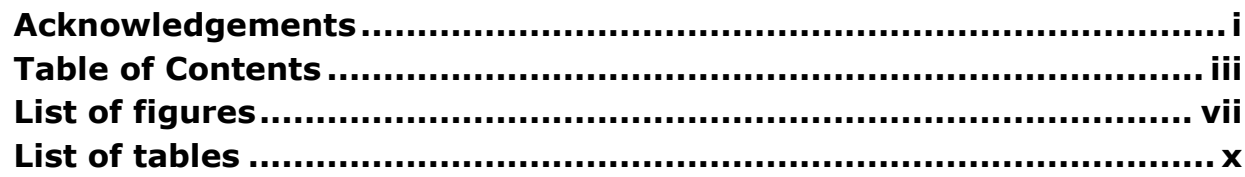

Chapter 1: General Introduction ............................................. 1

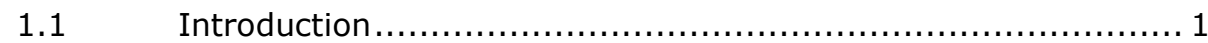

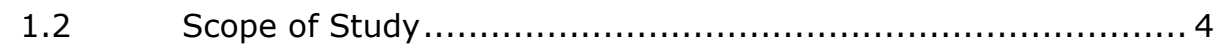

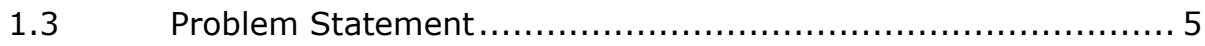

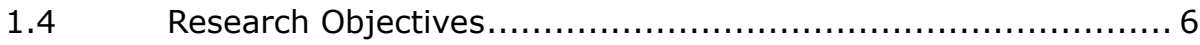

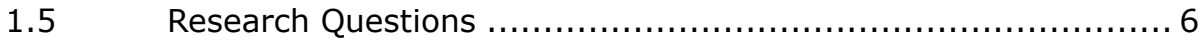

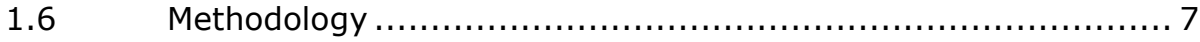

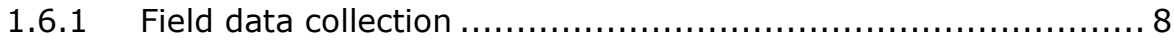

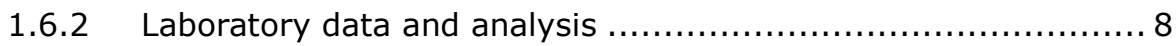

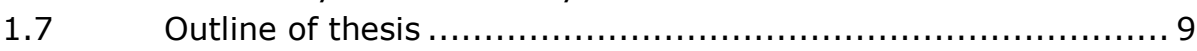

Chapter 2: Description of the study area................................... 11

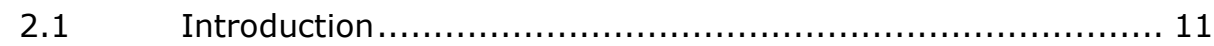

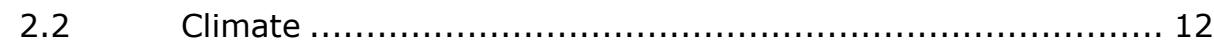

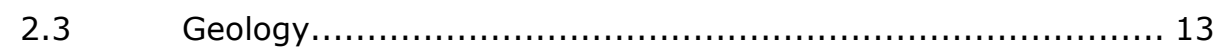

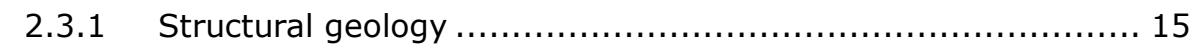

$2.4 \quad$ Weathering profiles over sedimentary bedrock $\ldots \ldots \ldots \ldots \ldots \ldots \ldots \ldots$

Chapter 3: Quantification of deterioration process in sandstone cut slope ............................................................................................. 17

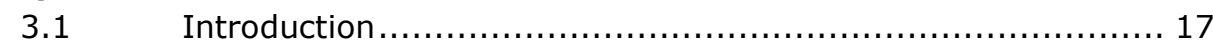

3.2 Material deterioration mechanism in slopes..................... 18

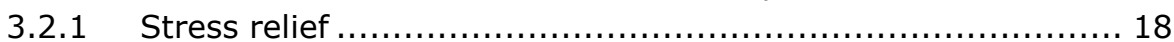

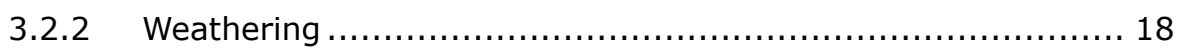

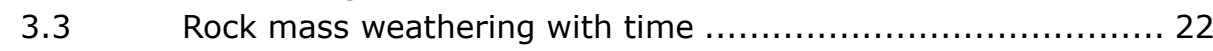

3.3.1 Weathering intensity and weathering intensity rate $\ldots \ldots \ldots \ldots \ldots 22$

3.3.2 Quantification of weathering in time............................. 23

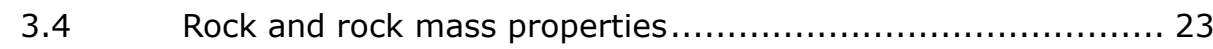

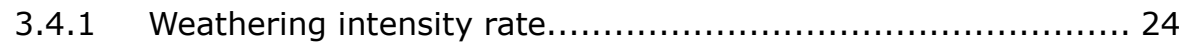

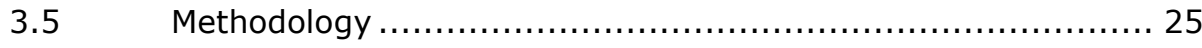

3.5.1 Rock mass cohesion and friction angle estimation .............. 27

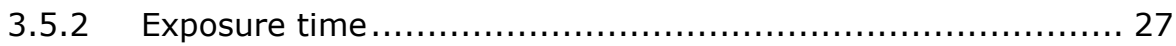

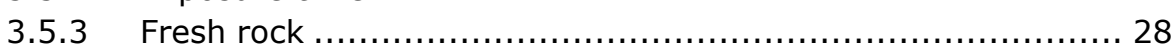

3.5.4 Weathering before and after excavation.......................... 28

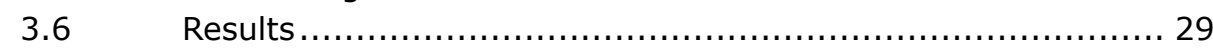

3.6.1 Correlation of intact rock strength determined by testing and "simple means" 
3.6.2 SST unit IRS and exposure time relation ......................... 32

3.6.3 Adjusted exposure time......................................... 33

3.6.4 Correlation between cohesion and friction angle of SST unit and exposure time ............................................. 35

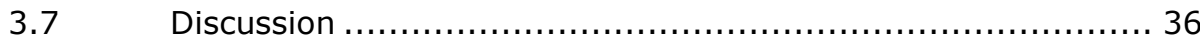

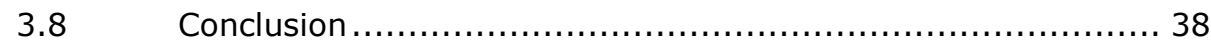

Chapter 4: Weathering effect on discontinuity properties................41

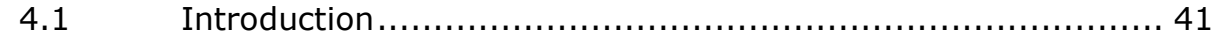

4.2 Rock mass discontinuities and weathering ....................... 42

4.2.1 Integral versus mechanical discontinuities ....................... 42

4.2 .2 Discontinuity Formation ........................................... 43

4.2.3 Discontinuity Sets and Properties.................................. 44

$4.3 \quad$ Methodology ......................................................... 46

4.3.1 Data Analysis ....................................................... 46

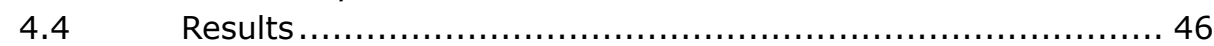

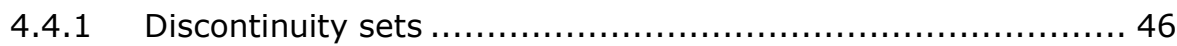

4.4 .2 Discontinuity spacing .............................................. 47

4.4.3 Discontinuity sequential development ............................ 48

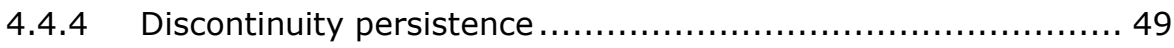

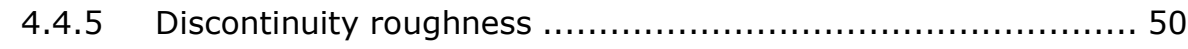

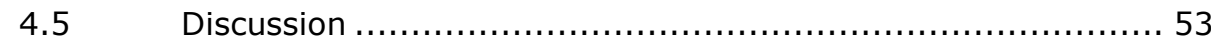

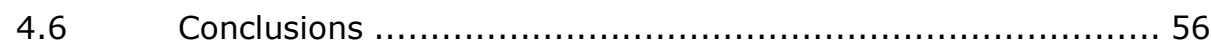

Chapter 5: Influence of weathering-induced iron precipitation on sandstone properties ................................................................... 59

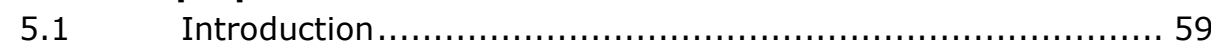

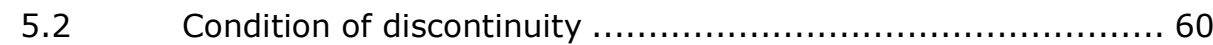

5.2.1 Barton's discontinuity inter-block shear strength .................60 60

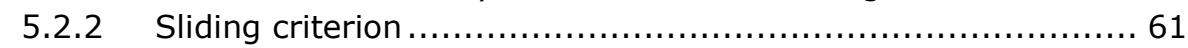

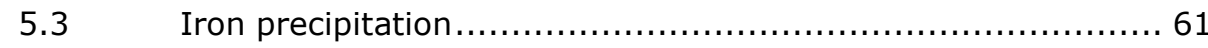

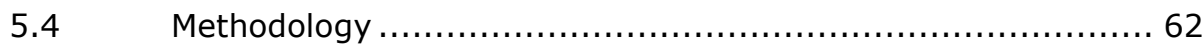

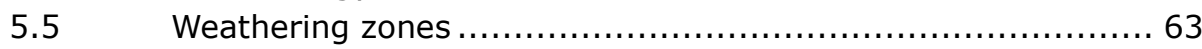

5.5.1 Weathering zone I ("surface oxidation") ................................ 63

5.5.2 Weathering zone II ("oxidation") ................................. 64

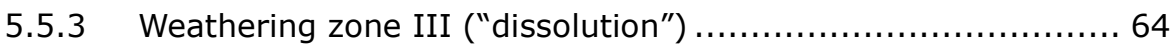

5.5.4 Weathering zone IV ("slightly weathered and fresh rock") ....... 65

5.6 Iron precipitation characteristics...................................... 65

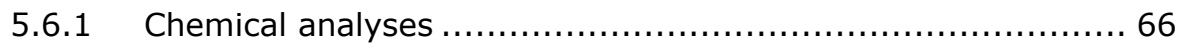

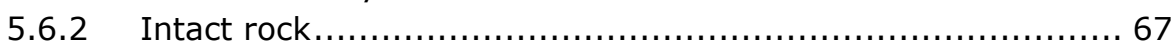

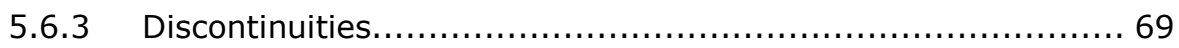

5.7 Iron precipitation effect on shear strength ..........................69 69

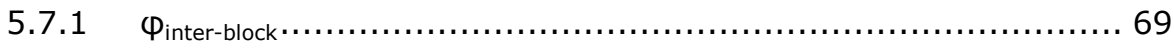

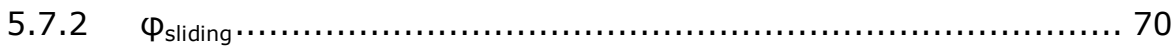




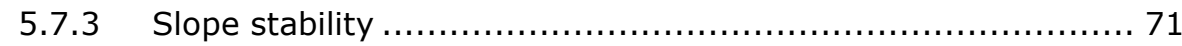

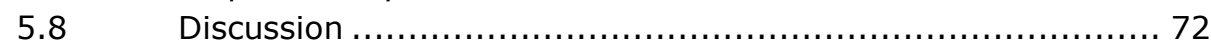

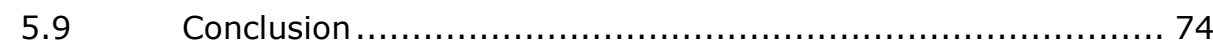

Chapter 6: Landslide susceptibility analysis...................................75

$6.1 \quad$ Introduction...................................................... 75

6.2 Previous landslide assessment study in Kota Kinabalu, Sabah . 77

$6.3 \quad$ Research methods ................................................ 78

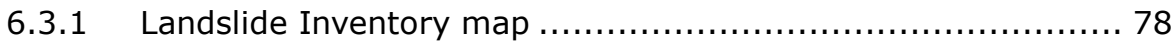

6.3.2 Bivariate Statistical Analysis........................................ 78

6.3.3 Validation of LSM and assessment of landslide causal parameters effects................................................... 80

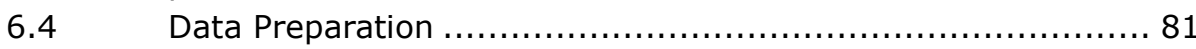

6.4.1 Landslide Inventory Map.......................................... 81

6.4.2 Selection of Landslide Preparatory Parameters ................... 82

6.4.3 Geological parameters................................................ 82

6.4.4 Topographical Parameters .......................................... 84

6.4.5 Anthropogenic Factors.............................................. 86

6.4.6 Hydrological Parameters .......................................... 88

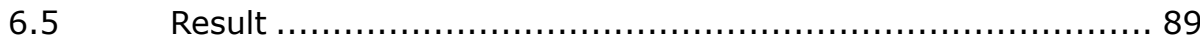

6.5.1 Landslide parameter/factor class weight .......................... 89

6.5.2 Assessment of landslide causal parameters effects and validation of LSM .................................................. 90

6.5.3 Landslide Susceptibility Map...................................... 91

6.5.4 Validation of Landslide susceptibility map ........................ 92

6.6 Discussion .......................................................... 93

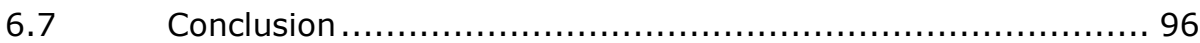

Chapter 7: Synthesis and Conclusions...............................................99

$7.1 \quad$ Synthesis ........................................................... 99

7.1.1 Objective 1: develop a quantitative factor for incorporation in the design of slopes to account for weathering................. 99

7.1.2 Objective 2: to evaluate the effect of weathering on the development of discontinuities in rock mass...............101

7.1.3 Objective 3: to assess the effect of iron solution and precipitation; and their influence on the stability of cut slopes.....

7.1.4 Objective 4: to assess the predictive power of several selected landslide causal parameter groups, which include the effect of deterioration processes (road excavation time) in the analysis of landslide susceptibility maps (LSM) for the northern part of Kota Kinabalu.... 


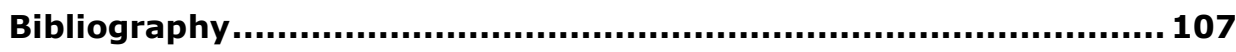

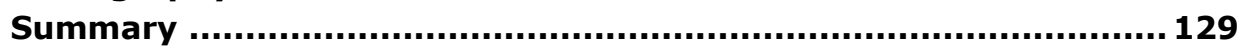

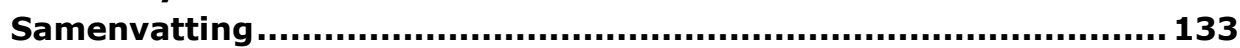

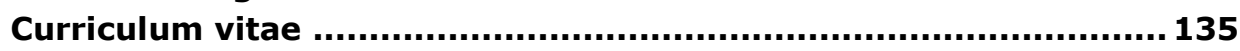

Publication related to this research ............................................ 136

ITC Dissertation List ............................................................... 137 


\section{List of figures}

Figure 1.1 Location of the research area (modified from Tating, 2003). 1

Figure 1.2 (a) Slope failure of a man-made slope causing collapse of part of buildings and structures on the slope and damage of the road surface (Zen Garden Resort, Kundasang Sabah); (b) Slope failure along a major road causing traffic disruption and hazard to the road users (KM 82.2 Tamparuli - Ranau Road, Sabah). 2

Figure 1.3 Deteriorating rock masses in a man-made slope along Ranau Tambunan road.

Figure 2.1 Topographical map of the research area (modified from Tating, 2003).

Figure 2.2 (a) Geological setting of the research area, (b) Detailed lithology of the study area (after Tating, 2003).

Figure 2.3 Exposure with typical geotechnical units in the Kota Kinabalu area.

Figure 2.4 Weathering profile of the thick sedimentary strata in the research area.

Figure 3.1 Weathering of intact rock. Note the formation of residual layer that will inhibit the weathering process towards the inner part of intact rock

Figure 3.2 Influence of weathering and stress relief on intact rock and rock mass properties (after Hack, 2011). 21

Figure 3.3 Weathering of rock mass. Weathering take place along the discontinuities and affected the discontinuity shear strength. 21

Figure 3.4 Definition of apparent and dynamic property change rates (Modified from Huisman, 2006).

Figure 3.5 Comparison between unconfined Compressive Strength (UCS) tests (from various reports, Table 3.3) and "simple means" tests

(Table 3.2) per rock mass weathering grade (Table 3.1). 32

Figure 3.6 IRS vs. exposure time of SST unit; a) logarithmic function; b) exponential function.

Figure 3.7 Observed IRS vs. estimated exposure time of SST unit; (a) logarithmic function; (b) exponential function. 34

Figure 3.8 Linear relation of IRS vs. logarithmic estimated exposure time. 35

Figure 3.9 Estimated cohesion vs. estimated exposure time of SST Unit. 35

Figure 3.10 Estimated friction angle vs. estimated exposure time of SST Unit.

Figure 3.11 Comparison with other similar studies on weathering over time; (a) Hachinohe et al. (2000) in sandstone at depth of $0.01 \mathrm{~m}$ below surface; (b) weathering intensity parameter (WE) over time (modified from Huisman, 2006). 
Figure 4.1 Equal area, lower hemisphere plot of discontinuity poles with Fischer distributions and mean discontinuity planes per set, all plotted on lower hemisphere in Dips (2012).

Figure 4.2 Discontinuity sets in the thickly to very thickly bedded SST unit in a cut slope.

Figure 4.3 a) Joint system in SST unit, some of the joints are cemented. SST unit bedding orientation is shown by the strike/dip symbol; b) Detailed joint system induced by weathering; c) Newly visible bedding plane in the SST unit (length of hammer $\approx 0.3 \mathrm{~m}$ ). $\quad 50$

Figure 4.4 Mean (a - d) and boxplot (e - h) of discontinuity spacing decreases with higher weathering grades. $M$ indicates median value in the boxplot.

Figure 4.5 a - d Plots of discontinuity poles per weathering grade; e - $\mathrm{h}$ schematic model showing the development of discontinuity sets in the steeply dipping SST unit.

Figure 4.6 Frequency of discontinuity spacing distribution per weathering grade for all discontinuity sets together.

Figure 4.7 Iron cemented joints in SST unit (length of hammer $\approx 0.3 \mathrm{~m}$ ). 54

Figure 4.8 Development of joint sets under a three-dimensional stress regime. The conjugate joint sets (set 2) and extensional joints (set 3) may be developed from the main principal stress ( $\sigma 1) .55$

Figure 5.1 Typical weathering profile in sandstone in the study area. The boundaries between the zones are gradual. The arrows indicate the direction of flow of solution products; weathering grade follows (ISO 14689-1: 2003).

Figure 5.2 Relict iron-precipitated discontinuities in sandstone; the arrows indicate examples of relict discontinuities extruding up to about 5 $\mathrm{cm}$ from the rock mass surface (length of hammer $\approx 0.3 \mathrm{~m}$ ). $\quad 64$

Figure 5.3 Iron precipitation types in discontinuities. 66

Figure 5.4 Development of discontinuities in oxidation zone (a-b) and dissolution zone (c-d). The iron content gradually decreases towards the dissolution zone or inwards into the intact rock. 67

Figure 5.5 Average chemical element content and density vs. rock mass weathering grade; $\mathrm{SiO}_{2}$ and $\mathrm{CaCO}_{3}$ content decrease, while $\mathrm{Al} 2 \mathrm{O} 3$ and $\mathrm{Fe}$ increase with higher grades of weathering. $\mathrm{SiO}_{2}$ content on left axis; contents of other elements on right axis. 69

Figure 5.6 Influence of different types of iron precipitation on the sliding angle (Equation 5.3) and inter-block friction angle (Equation 5.2) of discontinuities for moderately (grade 2) and highly (grade 3) weathered rock masses.

Figure 5.7 Iron precipitation in discontinuities reinforces the stability of a cut slope; susceptibility to slope degradation is low in rock mass with iron-precipitated discontinuities (IP) compared to rock mass without (NIP). 
Figure 6.1 Ideal block diagram of landslide. The GPS reading for the location of landslide is taken at the landslide crown area (after Varnes 1978).

Figure 6.2 Location of landslides.

Figure 6.3 Geological factor maps used for the landslide susceptibility assessment; a. lithology; b. distance to structural geology; c. structural geology density.

Figure 6.4 Topographical factor maps used for the landslide susceptibility assessment; a. elevation; b. aspect; c. slope angle.

Figure 6.5 Anthropogenic factor maps used for the landslide susceptibility assessment; a. distance to road; b. road excavation time; c. land use/land cover.

Figure 6.6 Hydrological factor maps used for the landslide susceptibility assessment; a. distance to river; b. drainage density. $\quad 88$

Figure 6.7 The comparison between "success rate curve" by using all parameter group and without a particular parameter group. The areas under the curve (AUC) for the different scenario are shown in the parenthesis.

Figure 6.8 Final susceptibility map obtained by using three landslide causal parameter groups, topographic, anthropogenic, and hydrological factors. The landslide inventory location is also shown in the map.

Figure 6.9 The "success rate curve" of the final landslide susceptibility map (green line). 


\section{List of tables}

Table 2.1 Description of rock units. The same units are used to define the geotechnical units (after Tating et al., 2013). 14

Table 2.2 Petrographic analysis of sandstone of the Crocker Formation (after Tating, 1997). 15

Table 3.1 Rock mass weathering grades. 26

Table 3.2 Field determination of intact rock strength (IRS) by "simple means" test. Modified from BS 5930:1981; BS 5930: 1999. 27

Table 3.3 Point Load Strength (PLS) and Unconfined Compressive Strength (UCS) for sandstone of the Crocker Formation. 30

Table 3.4 IRS values for thick to very thick-bedded sandstone (SST) unit. 31

Table 4.1 Discontinuity sets in SST unit. $\quad 47$

Table 4.2 Comparison of mean and median discontinuity spacing per set and weathering grade. "All" means that the values are for all sets together irrespective of to which set the plane belongs. $\quad 49$

Table 5.1 Result of the X-ray fluorescence (XRF) analysis. 68

Table 5.2 Major average chemical elements in intact rock per rock mass weathering grade. 68

Table 5.3 Iron content in the form of $\mathrm{Fe}_{2} \mathrm{O}$ and $\mathrm{Fe}_{2} \mathrm{O}_{3}$ per precipitation type in discontinuities (average in brackets).

69

Table 5.4 $\mathrm{Jr}$ and $\mathrm{Ja}$ values for iron-precipitated discontinuities with pinterblock. Jr and Ja values and descriptions following Barton et al. (1974).

Table 5.5 Summary of characteristics of iron precipitation in sandstone rock mass.

Table 6.1 Weight values obtained from the bivariate statistical method for all parameter classes.

Table 6.2 AUC values obtained from different analysis scenario of landslide susceptibility index (LSI). 


\section{Chapter 1: General Introduction}

\subsection{Introduction}

Many countries in the world undergo rapid urbanization. This requires more or less flat areas for residential and commercial structures and for infrastructure. In hilly and mountainous terrain, this leads to extensive levelling and slope excavation work. In many countries with limited resources, design of excavation work and slopes is not according to proper engineering standards. In particular, future stability of slopes is often not considered. Slopes may then be stable at time of excavation but become unstable within the so-called "engineering lifetime" of the slope. The engineering lifetime is the period a civil engineering structure, e.g. a slope, should be usable, which is mostly in the order of 50 to 100 years after construction. Instable slopes are a risk in urbanized areas, for users of infrastructure, and often cause large economic losses. In the area around Kota Kinabalu, Sabah, Malaysia (Figure 1.1), the flat areas around towns and cities suitable for housing and infrastructure are exhausted. Further development is necessarily into hilly terrain. Development in these areas requires extensive levelling of the terrain and excavation of slopes. Often the slopes are excavated without following proper engineering standards and measures to ensure future slope stability. Similar situations are quite common in many developing countries.
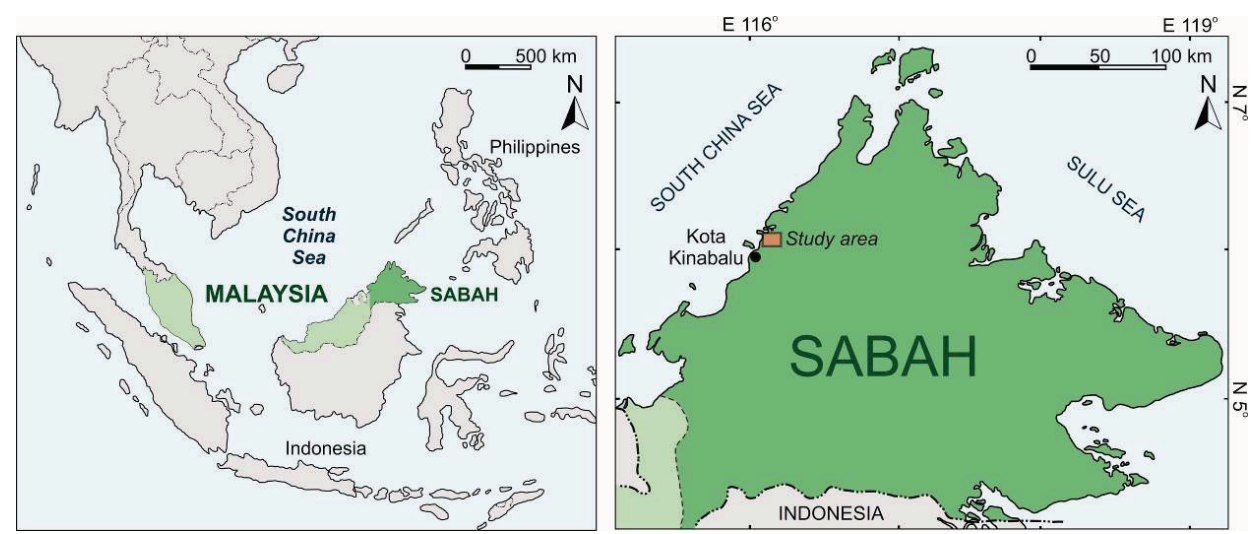

Figure 1.1 Location of the research area (modified from Tating, 2003).

Studies on slope failure incidents in Malaysia show that most slope failures occur in man-made slopes mainly along highways and in residential and urban areas (Figure 1.2) (Komoo et al., 2011; Qasim et al., 2013). Most of the incidents are the result of human factors such as insufficient or poor slope design, incompetency, construction material failure, and lack of maintenance (Qasim et al., 2013; Jamaludin \& Hussien, 2006; Gue and Tan, 
2006). Inadequacy and poor slope design are often related to the lack of understanding and appreciation of the present and future mechanical condition of the materials in which the slopes are made (Gue and Tan, 2006). The future mechanical condition is influenced by the deterioration process that progressively effects the durability of man-made slopes due to the effects of weathering and stress relief (Huisman, 2006; Tating et al., 2013).

(a)

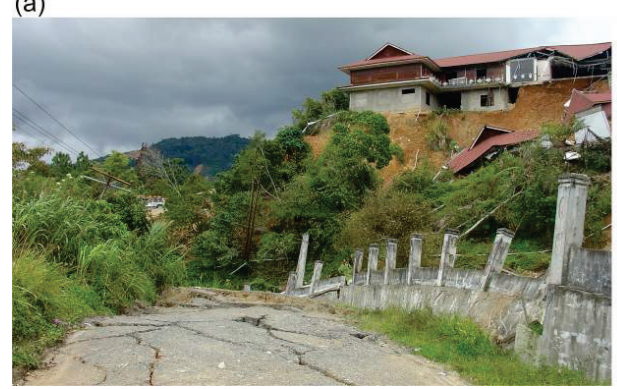

(b)

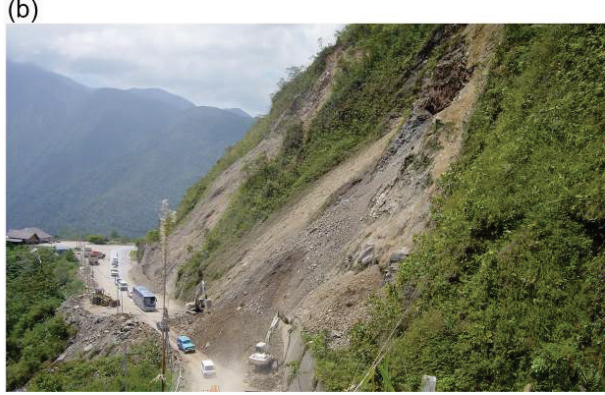

Figure 1.2 (a) Slope failure of a man-made slope causing collapse of part of buildings and structures on the slope and damage of the road surface (Zen Garden Resort, Kundasang Sabah); (b) Slope failure along a major road causing traffic disruption and hazard to the road users (KM 82.2 Tamparuli - Ranau Road, Sabah).

Weathering is defined as the alteration and breakdown of the groundmass ${ }^{1}$, i.e. soil or rock mass, under influence of Earth surface processes, such as ground- and surface water and temperature differences (Ollier, 1991) (Figure 1.3). Weathering is often a main agent in causing slope instabilities (Borelli et al., 2007; Hack, 1998, Miscevic and Vlastelica, 2014; Viles, 2013). Weathering causes a decrease of intact ground strength, an increase in the number of mechanical discontinuities ${ }^{2}$, formation of new discontinuities in intact material, and decrease of shear and tensile strength along discontinuities. It may also govern the development of different types of slope failure (Durgin, 1977; Calcaterra and Parise, 2005). Stress relief, which is the effect caused by the release of locked-in strain energy in groundmasses due to removal of overburden (Price, 1995), also results in opening and formation of new discontinuities and reduction of shear strength (Price, 1995; Hack, 1998; Hencher, 2013). The effects of weathering and stress relief on groundmasses can mostly not be differentiated, as both may

1 The terminology for ground-, soil, and rock masses is in accordance with the definitions used by De Mulder et al., 2012.

2 Mechanical discontinuities are planar features that act as a plane of weakness in a groundmass, such as bedding planes, joints, schistosity, cleavage, and faults. Mechanical discontinuities have a lower shear or tensile strength than the surrounding intact material. Groundmasses may contain integral discontinuities that are not yet a plane of mechanical weakness, such as bedding planes, but may change into a mechanical discontinuity under influence of, for example, stress changes or weathering (Hack, 1998, Price et al., 2009). 
happen at the same time, may influence or enhance each other, and result in identical effects. For example, the formation of new discontinuities due to stress relief may increase weathering by facilitating the percolation of groundwater in the groundmass. Both, weathering and stress relief effects are a function of time (Colman, 1981; Fookes et al., 1988; Price, 1995; Hencher, 2013). Development of new discontinuities and displacements along discontinuities due to stress relief may be also time dependent, because of continuous removal of overburden by erosion processes. Understanding the deterioration processes and their effect in time on the properties of both intact ground and groundmass is essential for a design of safe man-made slopes for the entire engineering lifetime of the slope.

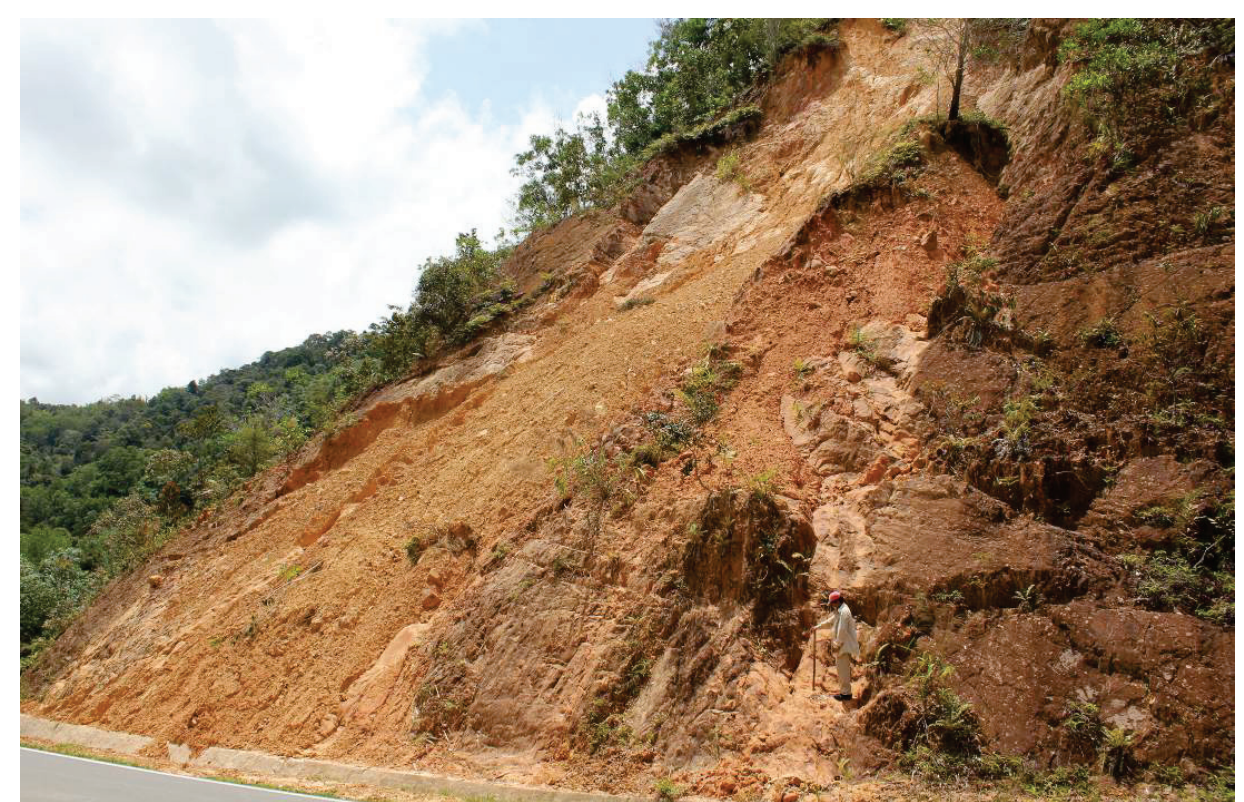

Figure 1.3 Deteriorating rock masses in a man-made slope along Ranau - Tambunan road.

In tropical humid regions, such as Kota Kinabalu, Sabah, Malaysia, manmade slopes may expose groundmasses that are already weathered. Hence, a slope cut in such a groundmass should be stable in the weathered groundmass as encountered in-situ and be stable in the groundmass with the added weathering in the future after exposure. In the practice of slope design in Malaysia, the required design engineering lifetime is 30 years or more, which is called the "serviceable lifetime". However, in Malaysia the design of slopes is based on using a suitable Factor of Safety (FoS) value, and on incorporating the existing weathering in a series of standards for slope design. The standards are based on the ratio of vertical height to horizontal length of a slope depending on the degree of weathering of the mass the slope is constructed in. For unweathered rock masses, i.e. weathering grade I 
following British Standard 5930:1981, the preliminary slope design parameter is $4: 1$ (vertical : horizontal) which gives a slope dip of about $76^{\circ}$. For weathering grade II rock masses, a ratio of $3: 1\left(\approx 72^{\circ}\right)$ is applied. If the slope shows signs of instability, it has to be redesigned to a lower slope dip and/or stabilization measures have to be applied (PWD, 2010). For weathering grades III to VI, the slope is designed based on a calculation of the Factor of Safety (FoS). A minimum FoS of 1.3 is generally required. Mitigation or stabilization measures can be applied where required.

The design criteria in Malaysia thus do recognize the different degrees of weathering as are at time of excavation as important for the design, however do not anticipate on degradation of the groundmass due to future weathering and stress relief. Therefore in this study, quantitative factors are developed that can be used in the design of slopes to account for future degradation of the groundmass. These factors are developed for thick-bedded sandstone layers. Although developed in Malaysia, the factors can likely also be used in other parts of the world with similar climate and slopes made in similar materials. Related studies have been done on the mechanism and development of new discontinuity patterns as function of progressive weathering, and the mechanism of iron precipitation and its influence on slope stability. A secondary related study has been done to the possibilities to use the time of exposure of man-made rock slopes without signs of failure as a parameter in the assessment of (natural) landslides ${ }^{3}$ in the area of Kota Kinabalu.

\subsection{Scope of Study}

Sedimentary rock strata may undergo rapid weathering in humid tropical climates when exposed. This causes a rapid degradation of the rock mass and reduction of geotechnical properties. The degradation may be significant within the engineering lifetime of a slope; hence, a slope may be stable at time of excavation but may become unstable in the future. Therefore, any design of civil engineering structures, such as slopes, that expose a rock mass have to be designed accounting for a reduction of geotechnical properties during the lifetime of the slope. At present, no quantified relations between degradation and time for sedimentary rock masses exist for use in humid tropical environments. If future degradation is taken into account at all in design, it is incorporated in an often arbitrarily higher factor of safety based on experience or literature, or it is guessed by the design or construction engineers. Design practice would improve if quantified factors

\footnotetext{
${ }^{3}$ Slope failure and landslide are used often as synonyms in the literature. In this study the term "slope failure" is reserved for failure of a man-made slope and "landslide" for failure of a natural slope.
} 
would be available incorporating the future degradation in the design calculations. Therefore, a study is done to relate the degradation of geotechnical properties to time for selected rock mass types in a humid tropical environment.

The study is done in the area around Kota Kinabalu, Sabah, Malaysia, and only focused on the thick to very thick-bedded sandstone (SST) units. This unit is the dominant lithology and closely related to landslide and slope failure incidents in this area (Tating, 2003). The area has a humid tropical climate with a uniform and high humidity throughout the year.

\subsection{Problem Statement}

Many authors, e.g. Hack (1998), Huisman (2006) and Viles (2013), have suggested further research to relate weathering and slope stability and possibilities for quantification of weathering influence on geotechnical properties. Existing weathering classifications are mainly qualitative, e.g. BS5930 (1981, 1999), ISO 14689-1 (2003), ASTM D5878-08 (2008). Some attempt to quantify weathering by using the ratio of unsound to sound constituents' of rock (Irfan and Dearman, 1978) and the ratio of weathered to unweathered minerals (Selby, 1993) have been proposed, however these cannot be applied directly to slope design. The factors developed by Hack (1998) and Hack et al., (2003) can be used for design but are not quantified in time. Huisman (2006) developed factors for deterioration in time in a Mediterranean climate. In the study described in this thesis, weathering of bedded sandstone is quantified in time and results in factors that can directly be used in slope design in a tropical humid climate.

The formation of new discontinuities due to weathering and stress relief (Ehlen, 2002; Fell et al., 2012; Hack and Price 1997; Hencher, 2013) is well known in most type of rock; however, the formation mechanism and resulting variation of newly formed discontinuities throughout a rock mass especially in sedimentary rocks such as sandstone in a tropical environment are often poorly understood. In this research, the interrelation between weathering and mechanisms responsible for forming discontinuities have been investigated, motivated by the necessity to assess the engineering implications of mechanical discontinuity sets that are not a mechanical discontinuity set in a rock mass with lower weathering degree but will become a mechanical discontinuity set as a consequence of further weathering.

During certain chemical weathering processes in rock masses, sequential interaction between percolating groundwater and rock will result in the development of oxidation and dissolved zones (Chigara and Oyama, 1999; Chigara, 1990). The development of weathering zones will influence the 
precipitation of iron within the rock mass and also along the discontinuities. Weathering-induced iron dissolution and precipitation are important processes that govern the geotechnical properties of discontinuities and intact rock in some rock masses. Iron dissolution and precipitation are well known, but the influence on mechanical properties of rock masses is rarely reported in the literature. Therefore, the characterization, mechanism, and effect of iron dissolution and precipitation as consequence of weathering on properties of sandstone especially in tropical area are presented in this research.

Rock masses in man-made slopes will begin to respond to the disturbance caused by the excavation and the new environment as soon as they are excavated. The disturbance often causes that the existing (natural) slope in which the slope cut is made, also is disturbed. This may cause deterioration of the mass and instability of the existing (natural) slope. Often this process is referred to as progressing "loss of structure" in the existing (natural) slope (Hack, 1998). Therefore, existing (natural) slopes with a man-made cut slope in it are often more susceptible to landslides outside the man-made cut area. The period between the excavation of the first man-made excavated slope and the time of investigation of landslides in the natural slope outside the man-made cut area may be used as an indicator for the forecasting of the stability of the (natural) slope. This time factor is used as one of the parameters in making of a Landslide Susceptibility Map (LSM) of the Northern Kota Kinabalu area.

\subsection{Research Objectives}

In order to be able to design slopes that are stable during the full engineering lifetime of the slope the objectives of the research are:

- to develop quantitative factors for incorporation in design of slopes that account for degradation of natural materials due to stress relief and weathering

- to determine the effect of weathering on the development of discontinuities in rock masses

- to assess the effect of iron solution and precipitation; and its influence on the stability of man-made slopes

- $\quad$ to assess the predictive power of several selected landslide causal parameter groups, which include the effect of deterioration process (road excavation time) in the analysis of landslide susceptibility map (LSM) for the northern part of Kota Kinabalu

\subsection{Research Questions}

In relation to the problem statement and the main objectives, the research is done to endeavour answers to the following questions concerning weathering and deterioration of sandstone rock masses in a humid tropical environment: 
1. How to quantify deterioration processes of rock masses that can be incorporated into slope design?

- How is the quantification to be established?

- What are the rock properties important in quantification of deterioration processes?

- How should the quantification be used in man-made slope design?

2. What is the effect of deterioration processes on the development of new discontinuities in rock masses?

- How do deterioration processes initiate formation of new discontinuities?

- How do the new discontinuities develop in relation to progressing weathering?

- $\quad$ Can the future pattern of newly formed discontinuities due to the deterioration processes be predicted?

3. What is the consequence of chemical weathering on the rock mass and the stability of man- made slopes?

- What is the effect of chemical weathering on the characteristics and properties of a rock mass?

- What are the conditions for iron precipitation in rock mass?

- What is the effect of iron precipitation on rock slope stability?

4. What are the appropriate landslide causal parameter groups or factors that can be used in the development of landslide susceptibility map (LSM) at regional scale in the study area?

- What is the relative importance of landslide causal parameter groups or factors in the development of LSM?

- What is the appropriate combination of landslide causal parameter groups or factors for optimum accuracy of the LSM?

- Can excavation duration time contribute to the reliability of landslide susceptibility assessment?

\subsection{Methodology}

The research deals with the assessment of sandstone deterioration (weathering and stress relief) in a humid tropical environment. Also are determined and evaluated deterioration - time relationships, development of discontinuities, assessment of iron precipitation in rock masses, and development of a landslide susceptibility map. Therefore, the thesis is organized into chapters that address these specific topics, especially related to problems, review of relevant literature and methodology. Except for the development of landslide susceptibility map, the field data for the other components are similar and collected based on extensive and detailed field observation. 


\subsubsection{Field data collection}

Most of the rock exposures are easily accessible and therefore data could be collected reliably and exactly at the intended locations.

Characterization of rock mass is done following the Slope Stability Probability Classification (SSPC) method proposed by Hack et al. (2003). In this approach, the rock mass was firstly delineated into several geotechnical units (define as a certain portion of rock mass that exhibit similar mechanical properties), based on bedding thickness, lithological type, bedding thickness ratio between different lithology and weathering grade.

Subsequently, the intact rock strength (IRS) and discontinuity sets are characterized for each geotechnical unit. IRS was estimated by "simple means" field test method, which used hand and geological hammer based on IRS estimation procedure described in BS5930 $(1981,1999)$ and Hack and Huisman (2002) (see Table 3.2). Characterization of discontinuities is done based on the rapid face mapping approach (Slob, 2010). In this approach, the main or representative discontinuity sets are identified visually in the exposure and their mean orientation (dip/dip-direction) is determined by measuring several discontinuities for each set.

The discontinuity sets are further characterized based on orientation, spacing, persistence, roughness, infill, and whether karst occurs along the discontinuity sets. The discontinuity surface roughness is characterized based on visual and tactile (feeling with finger tops) assessment. The large-scale roughness is estimated visually on a discontinuity surface area between $0.2 \mathrm{x}$ $0.2 \mathrm{~m}^{2}$ and $1 \times 1 \mathrm{~m}^{2}$ in five classes; wavy, slightly wavy, curved, slightly curved and straight (following example traces given in SSPC). The small-scale roughness is described on an area of about $0.2 \times 0.2 \mathrm{~m}^{2}$ in three classes; steeped, undulating and planar (also following the SSPC). The small-scale roughness is further described with tactile roughness and described as rough, smooth, or polished. Other parameters include the characteristics of infill material and the presence of karst structures. Further, the method of excavation (ME) and the degree of rock mass weathering (WE) are characterized. Weathering grade of rock mass is determined visually similar to methodology described in BS5930:1981.

\subsubsection{Laboratory data and analysis}

Intact rock samples from each weathering grade and zone, and samples of precipitated material in discontinuities have been collected to determine the major element concentrations and in particular the iron content. Samples are analysed with a portable X-ray fluorescence instrument (Niton XL3t) in a laboratory set-up with helium purging. The duration of the XRF 
measurements is about 85 seconds per sample with filter settings specifically to determine rock-forming elements. After the XRF analysis, the dry specific densities of the rock samples were determined in each weathering grade.

\subsection{Outline of thesis}

The thesis comprises of a collection of research papers submitted or published in peer review international journals and conferences (chapters 3 (Tating et al., 2013a; 2013b; 2013c); chapter 4 (Tating et al., 2015a); chapter 5 (Tating et al., 2015b)) with additionally an introduction, chapter on the description of the study area, and concluding chapter (chapters 1, 2 and 7). Chapter 6 describes the use of an exposure time parameter in the assessment of landslide susceptibility. An article on this topic is under preparation. The thesis consists thus of:

Chapter 1 (Introduction) introduces the research. It consists of the background of the research, research problem, and objectives. The research methodology especially for the data collection is also elaborated on in this chapter.

Chapter 2 (Study area) describes the study area. It provides the description of the topography, environment, geology, and geotechnical characteristics of the rock masses.

Chapter 3 (Quantification of deterioration processes influencing the stability of man-made slopes in sandstone) provides a review of material deterioration mechanisms, rock mass weathering, and stress relief with time and its influence of the stability of man-made slopes in sandstone.

Chapter 4 (Weathering effect on discontinuity properties) provides a review of weathering effects on the rock mass discontinuities that include the development of new discontinuities sets related to weathering degree of rock masses.

Chapter 5 (Influence of weathering-induced iron dissolution and precipitation on sandstone properties) describes the influence of iron dissolution and precipitation on the condition of discontinuities in thickly to very thickly-bedded sandstone. The chapter also provides the processes controlling iron dissolution and precipitation in rock mass discontinuities in a tropical environment.

Chapter 6 (Landslide susceptibility assessment using bivariate statistical method) provides the development of landslide 
susceptibility maps for northern Kota Kinabalu area based on the appropriate combination of landslide causal parameters groups or factors at regional scale. The relative importance of landslide causal parameters groups was also determined for the study area. Apart from the common landslide causal parameter maps (slope, aspect, geology, land use, distance from structure, distance from road and distance from river), slope excavation time is used in susceptibility analysis which is the rock slope excavation time parameter related to the result of rock mass deterioration with time in chapter 3.

Chapter 7 (Synthesis and Conclusions) concludes the thesis with a summary reflecting the research objectives and findings, and recommendations for future research are presented. 


\section{Chapter 2: Description of the study area}

This chapter describes the area, geographic setting, and geology, geotechnical and weathering characteristics of the sedimentary rock. It is based on the published articles: "Engineering aspects and time effects of rapid deterioration of sandstone in the tropical environment of Sabah Malaysia" (Tating et al., 2013a) and "Weathering effects on discontinuity properties in sandstone in a tropical environment: case study at Kota Kinabalu Sabah, Malaysia" (Tating et al., 2015a). Part of the text may be similar to the text in the articles.

\subsection{Introduction}

The research area is located to the North of Kota Kinabalu in Sabah, East Malaysia (see Figure 1.1). It is located approximately between longitude $\mathrm{E}$ $116^{\circ} 06^{\prime}$ to $\mathrm{E} 116^{\circ} 14^{\prime}$ and latitude $\mathrm{N} 006^{\circ} 02^{\prime}$ and $\mathrm{N} 006^{\circ} 07^{\prime}$ in between the South China Sea to the west and the Crocker Range to the east. The study area covers approximately $140 \mathrm{~km}^{2}$.

The Western part of the area consists of rather flat swampy areas, coastal plains, valleys, and some isolated hills (Figure 2.1). The coastal plains and valleys vary from 2 to 5 kilometres in width while the linear belt of hills is about a kilometre wide. The coastal terrains and valleys are underlain by Quaternary deposits that consist of unconsolidated to semi-consolidated sedimentary layers of sand, silt, clay, and peat. The Eastern part consists mainly of series of parallel linear ridges mostly oriented about NE - SW. The ridges range from 30 to more than $800 \mathrm{~m}$ in height toward the eastern part of the area. The highest peak of the ridge is about $850 \mathrm{~m}$ above Mean Sea Level (MSL).

The accessibility within the study area is quite good. A good road network (sealed and unsealed) connects most of the towns and villages in the study area. The western part of the area has undergone rapid urban development, where many hilly areas are cleared from bush and woodland, and most of the lowland and swampy areas are reclaimed and filled for new housing development and infrastructure. This development provided many wellexposed rock exposures for the study described.

The majority of the data for the study has been collected from man-made slopes with different dates of excavation in the Kota Kinabalu Industrial Park (KKIP) area, which is located within the study area. This is a hilly area of about 1500 acres, cleared from vegetation since 1995. In addition to some already existing roads in the area and vicinity, many new roads with roadcuts have been made in the area between 1995 and 2011. Data of unweathered 
rock masses has also been collected from three quarry sites in the vicinity of the KKIP area. The dates of excavation of the roadcuts are well documented providing a good time control for the data. The dimensions of the slopes range from less than one metre to more than 80 metres in height and three to over 50 metres in width. Data from 97 man-made slopes are used for the study. The data has been collected during two fieldwork campaigns; March through May 2010 and April through June 2012.

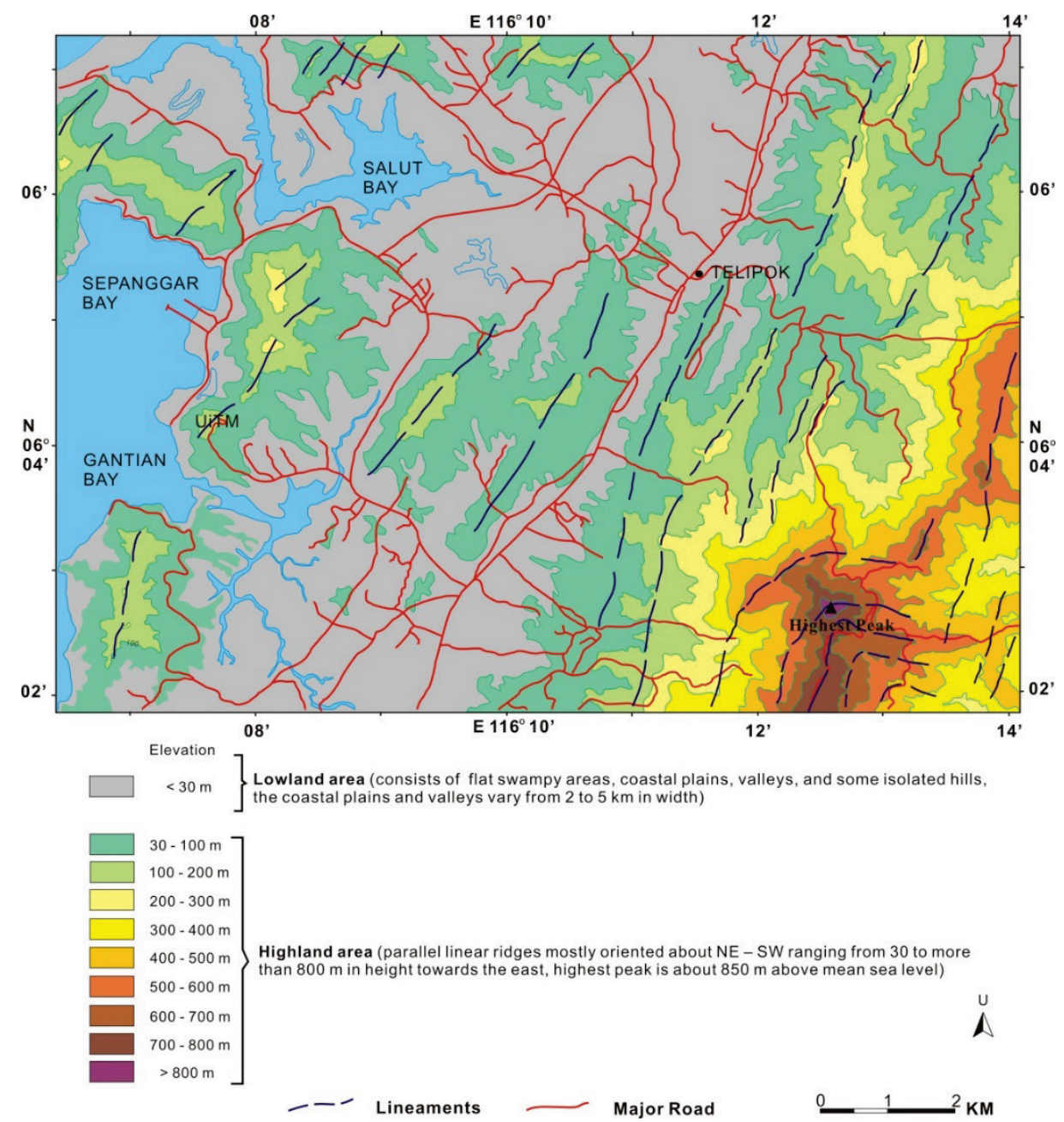

Figure 2.1 Topographical map of the research area (modified from Tating, 2003).

\subsection{Climate}

The area is in a typical tropical climate, which has a uniform temperature and high humidity throughout the year; classified as "equatorial-fully humid (Af)" following the Köppen-Geiger updated climate classification (Kottek et al., 
2006). The daily temperature ranges from $22^{\circ}$ to $33^{\circ} \mathrm{C}$ (average $27^{\circ} \mathrm{C}$ ) and the temperature variation throughout the year is small. The average humidity ranges from $79-85 \%$. The annual rainfall ranges from 1,920 to $3,190 \mathrm{~mm}$ (average $2,075 \mathrm{~mm}$ ). The temperature and annual rainfall are influenced by two prevailing monsoons. The north eastern monsoon during November to March results in cooler temperatures and less rainfall, while temperatures and rainfall are higher during the south western monsoon from May to September (MMD, 2013).

\subsection{Geology}

The geology in the area consists of a thick sequence of Eocene-Oligocene grey to bluish grey fine to medium grained sandstone and red and/or grey shale beds belonging to the "Crocker Formation" (Collenette, 1958) (Figure 2.2). The strata are trending towards the north - northeast or towards the northeast (NNE or NE) and are steeply dipping either to the east or west.
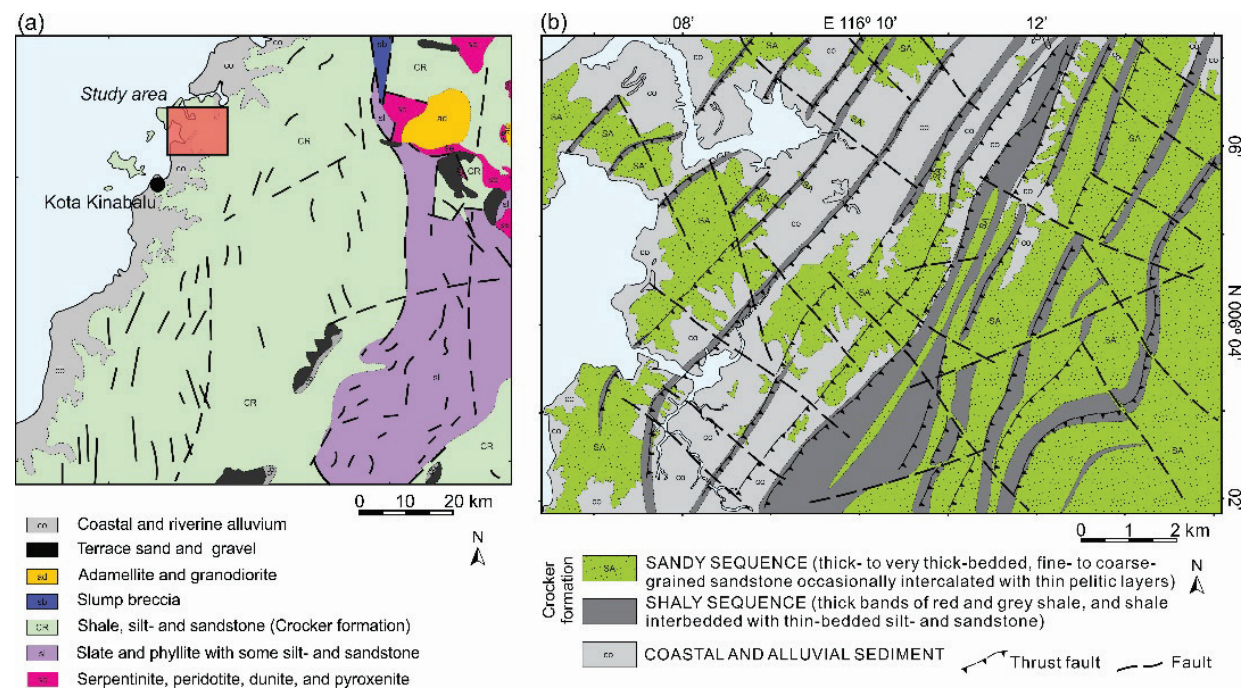

Figure 2.2 (a) Geological setting of the research area, (b) Detailed lithology of the study area (after Tating, 2003).

The rock sequence can be divided in three main lithological units based on the bedding thickness, sandstone to shale ratio, sedimentary structure characteristics, rock type and color (Tating, 2003). These parameters were also used to determine the geotechnical units for the rock mass classification (Table 2.1 and Figure 2.3). The lithological units are a thick to very thick bedded (sometimes massive) sandstone unit (SST Unit), a unit consisting of sandstone interbedded with shale (IB Unit) and grey shale or red shale unit (SH unit). 
Table 2.1 Description of rock units. The same units are used to define the geotechnical units (after Tating et al., 2013).

\begin{tabular}{|c|c|c|}
\hline Formation & Units & Description \\
\hline \multirow[t]{3}{*}{$\begin{array}{l}\text { Crocker } \\
\text { (Eocene to } \\
\text { Oligocene) }\end{array}$} & $\begin{array}{l}\text { Thick to very thick } \\
\text { bedded Sandstone } \\
\text { (SST) }\end{array}$ & $\begin{array}{l}\text { Bedding spacing from } 0.6 \text { to over } 2 \mathrm{~m} \text { with } \\
\text { some bedding spacing exceeding } 8 \mathrm{~m} \text {. Grey to } \\
\text { bluish grey in color. Fine- to coarse-grained } \\
\text { with locally sparse pebbles at the base. } \\
\text { Intercalated with thin pelitic layers with ratio } \\
\text { of } 9: 1 .\end{array}$ \\
\hline & $\begin{array}{l}\text { Interbedded } \\
\text { Sandstone, Siltstone } \\
\text { and Shale (IB) }\end{array}$ & $\begin{array}{l}\text { Interbedding between sandstone, siltstone } \\
\text { and shale beds. Bedding spacing from } 0.01 \text { to } \\
0.6 \mathrm{~m} \text {. Sandstone and pelitic layer ratio } \\
\text { varies. Divided into three subunits based on } \\
\text { ratio: Classical type (ratio } 1: 1) \text {, Shaly type } \\
\text { (ratio } 1: 3 \text { ) and Sandy type (ratio } 9: 1) \text {. }\end{array}$ \\
\hline & Red/Grey Shale $(\mathrm{SH})$ & $\begin{array}{l}\text { Red and Grey shale bands. Thickness from } \\
0.6 \text { to over } 25 \mathrm{~m} \text {. Red shale characterized by } \\
\text { distinctive red to brownish red color and } \\
\text { homogeneity. Occasionally interbedded with } \\
\text { siltstone. Grey shale consists of thinly } \\
\text { interbedded between grey shale, siltstone or } \\
\text { fine-grained sandstone }(1-20 \mathrm{~cm}) \text {. Shale and } \\
\text { sandstone ratio from } 3: 1 \text { to } 10: 1 \text {. }\end{array}$ \\
\hline
\end{tabular}

The stratigraphic succession of the thick sedimentary strata in Kota Kinabalu can be divided further into two main sequences based on the lithological dominance; i.e. "Sandy sequence" and "Shaly sequence" (Tongkul, 1987; Tating, 2003) (Figure 2.2b). The Sandy sequence consists predominantly of sandstone from the SST and IB units, whereas the Shaly sequence consist mainly of the $\mathrm{SH}$ units. The lithological units show a good lateral continuity and uniform thickness, and can be traced along strike for several kilometers.

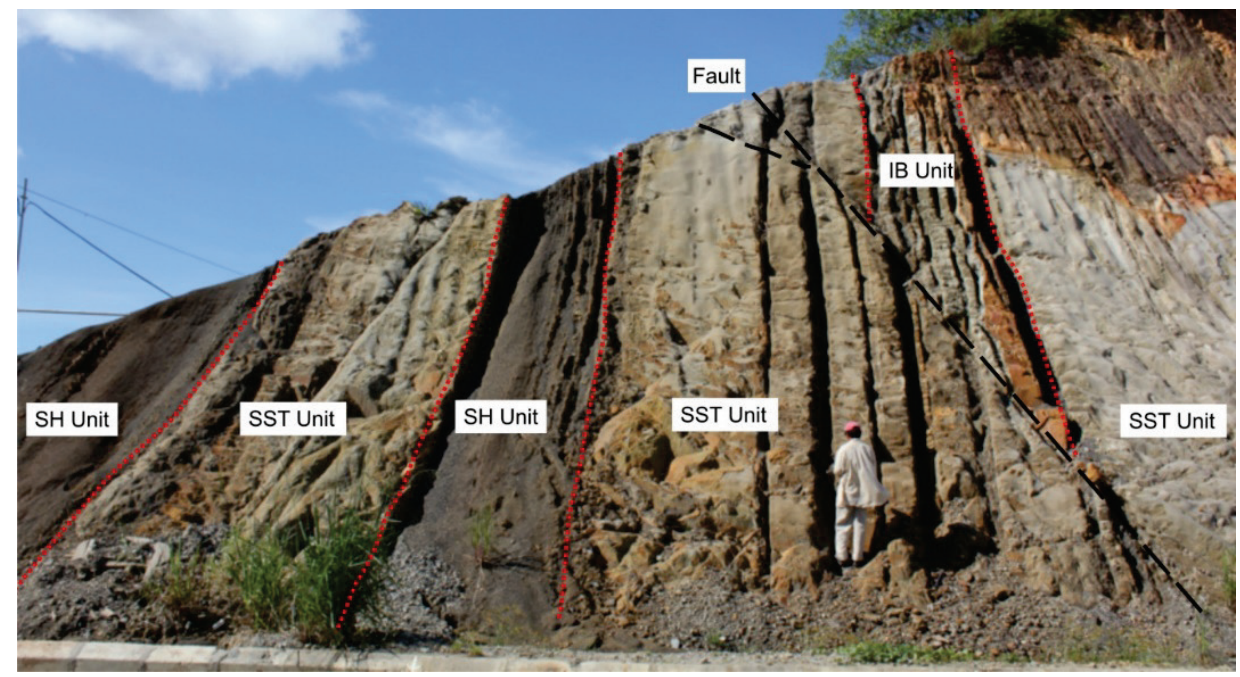

Figure 2.3 Exposure with typical geotechnical units in the Kota Kinabalu area. 
Thin section petrographic analyses of the sandstone show that the main constituents are quartz grains with subordinate amounts of lithics, feldspar, calcite, and opaque minerals embedded in a siliceous matrix with few voids (Table 2.2). The sandstone is medium-grained and poorly sorted. The quartz is present as discrete detrital grains and as polycrystalline aggregates. Most lithic fragments consist of chert with minor quantities of fragments consisting of shale and other siliceous igneous rocks. Pyrite $\left(\mathrm{FeS}_{2}\right)$ minerals are commonly associated with the sedimentary rock strata in the Kota Kinabalu area (Burgan \& Ali, 2009; Hutchison, 2005; Stephens, 1956; Van Hattum et al., 2003). It normally occurs as framboidal pyrites, but also often in the form of larger minerals visible to the naked eye.

Table 2.2 Petrographic analysis of sandstone of the Crocker Formation (after Tating, 1997).

\begin{tabular}{|c|c|c|c|c|c|c|c|}
\hline \multirow{2}{*}{$\begin{array}{l}\text { Sample } \\
\text { (\& Location) }\end{array}$} & \multicolumn{7}{|c|}{ Mineral Composition (\%) } \\
\hline & Quartz & Lithic & Feldspar & Calcite & Matrix & Opaque & Voids \\
\hline S1 (Telipok) & 60 & 15 & 4 & - & 20 & 1 & rare \\
\hline S2 (Menggatal) & 50 & 10 & 3 & 5 & 30 & 2 & rare \\
\hline S3 (Penampang) & 60 & 10 & 3 & 5 & 20 & 2 & rare \\
\hline
\end{tabular}

\subsubsection{Structural geology}

Generally, the structural features in the western part of Sabah and particularly the study area are dominated by a series of northeast (NE) southwest (SW) trending parallel linear lineaments. The lineaments are mainly representing major faults (thrust, normal and wrench faults) in thick sedimentary rock. The strata is subsequently imbricated into a series of fault blocks by tectonic activity during the Late Oligocene to Early - Middle Miocene and resulted in the present repetition of the lithological units at surface (Tongkul, 1990).

\subsection{Weathering profiles over sedimentary bedrock}

In general, weathering profiles over sedimentary strata in the area show a large lateral and vertical variability of weathered material and morphological features. This is due to changes in lithology, bedding thickness, and discontinuity orientation (Figure 2.4). The variability is increased by structural discontinuity planes, such as joint and fault planes. These discontinuity planes may govern the flow of subsurface water, which can lead to selective weathering and variability in the precipitation of minerals, in particular iron minerals. The thickness of the weathering profiles varies and ranges from 3 to more than 30 metres. The most obvious visual characteristics are the presence of two zones with distinctive colour. The upper part consists of brownish coloured weathered rock material (oxidation 
zone) whereas the lower part is mostly grey to greyish (dissolved zone) (further discuss in Chapter 5).

In the upper part, the most dominant feature is the precipitation of iron along the discontinuities due to oxidation. "Iron pans" or "duricrusts" may be present due to the accumulation of iron and aluminium oxide. Iron precipitation especially in the thick to very thick-bedded SST unit occurs as staining, coating, and cementing. The precipitation and mechanism responsible are described in detail in Chapter $\mathbf{5}$.

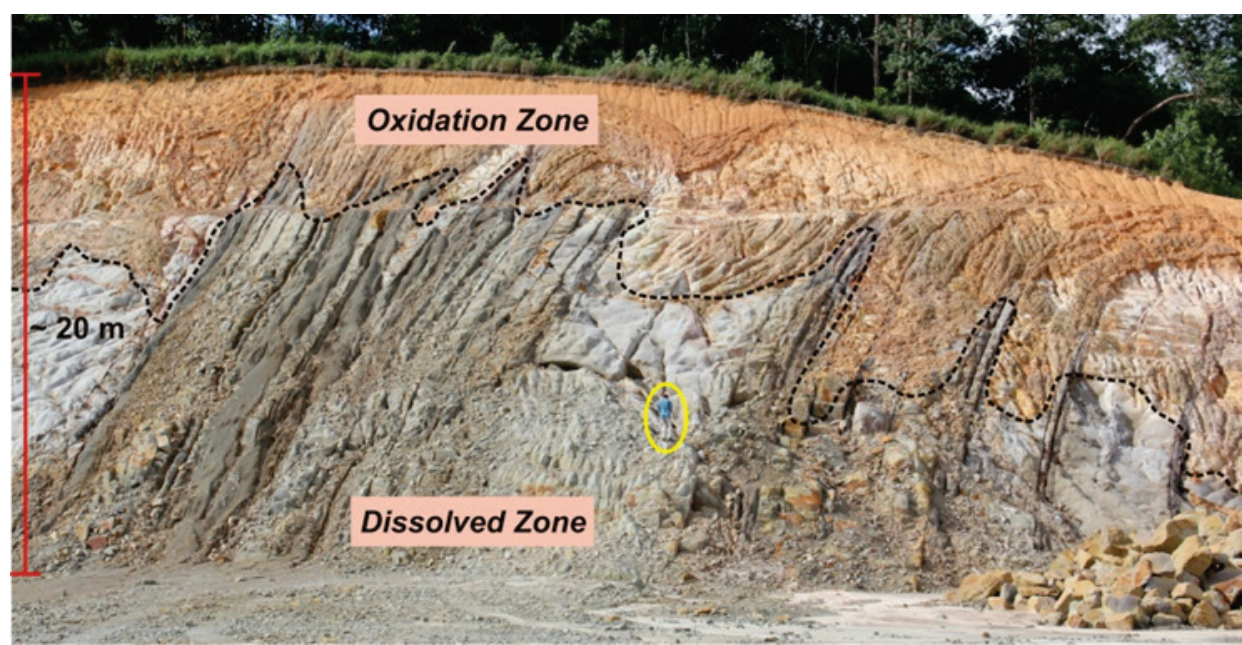

Figure 2.4 Weathering profile of the thick sedimentary strata in the research area. 


\section{Chapter 3: Quantification of deterioration process in sandstone cut slope}

This chapter described the development of deterioration (weathering and stress relief) quantitative and their incorporation in slope design. This is based on the articles "Engineering aspects and time effects of rapid deterioration of sandstone in the tropical environment of Sabah, Malaysia" (Tating et al., 2013a); "Weathering and deterioration as quantitative factors in slope design in humid tropical areas: case study Northern Kota Kinabalu, Sabah, Malaysia" (Tating et al., 2013b); and "Quantification of rock mass deterioration processes for cut slope design in humid tropical areas - case study Northern Kota Kinabalu, Sabah, Malaysia" (Tating et al., 2013c), therefore to some extend the text are the same.

\subsection{Introduction}

Rock mass or ground mass can be defined as a volume of ground that will be affected by an engineering works (Price et al., 2009). Rock mass consist of an assemblage of rock blocks (intact rock) dissected by various sets of discontinuities. The presence of discontinuities will reduces the strength and increases the deformability of the rock mass; and may also influence the weathering mechanism and pattern within the rock mass. Any engineering works on rock mass such as roadcut may result in further deterioration (stress relief and weathering) of rock mass material that will lead into slope instability.

Roadcut slopes in Sabah Malaysia are designed to be stable over a certain time span, normally more than 30 years, which is the "serviceable lifetime" (Chua, V.; personal comm. June 1, 2012). The "serviceable lifetime" of the slope is achieved by using a higher Factor of Safety (FoS) than required for a stable design at the time of construction. Although the design is supposed to result in a slope to be stable for more than 30 years, i.e. an engineering lifetime of 30 years, many slopes or parts of slopes fail within 30 years after construction. The reasons for failure sometime after construction but before the design "serviceable life" time ends are likely stress relief and weathering. Throughout the world, stress relief and weathering are often marginally quantified or simply neglected in slope design (Wong et al., 1998; Huisman et al., 2006; Gue and Tan, 2006).

A proper design of a slope for the entire engineering lifetime of the slope should include quantitative factors accounting for the degradation of the rock mass over its lifetime. However, quantified relations over engineering periods are seldom in the published literature and do not exist for the tropical climate and environment of Malaysia. Most of the published studies on rock 
weathering concern the influence of weathering on the geotechnical properties of the intact rock in engineering applications, and the development of weathering profiles and soil layers over long (geological) time spans (e.g. Raj, 1985; Mohamed et al., 2007; Mohamad et al., 2008, 2011; Ghiasi et al., 2009; Chigira et al., 2011). In this research quantitative factors are developed for incorporation in the design of slopes to account for stress relief and weathering in humid tropical areas.

\subsection{Material deterioration mechanism in slopes}

Stress relief and weathering are the dominant deterioration processes that affect the durability of a man-made rock slope after excavation. These cause the physical, geotechnical and chemical properties of the slope material to change in response to the new environment and are considered a major cause of future geotechnical engineering problems (Hack and Price, 1997).

\subsubsection{Stress relief}

The excavation of a slope causes a change in stress regime in the rock mass forming the slope; generally, stresses will become less due to the removal of material, however, stress increases and stress concentrations may occur as well. Stress reduction allows opening of existing discontinuities, and stress increase and changes in stress concentrations may lead to new discontinuities as may be stress relief fracturing in shaly type of rocks (Price et al., 2009). Moreover, the change in stress field may cause new discontinuities because of reduction of confining pressure (Anon., 1995; Hudson and Harrison, 1997; Hencher and Knipe, 2007). The new and more opened existing discontinuities allow for a higher permeability and hence an increase in water permeating into and flowing through the rock mass. Weathering will then increase and be faster as water is one of the main agents involved in weathering (Bland and Rolls, 1998; Huisman, 2006).

\subsubsection{Weathering}

Weathering is the chemical and physical break down of minerals due to physical, chemical, and biological processes. The weathering affects intact rock but also the fabric and nature of discontinuities in the rock mass (Gupta and Rao, 2001; Nicholson, 2004; Gurocak and Kilic, 2005; De Mulder et al., 2012). Weathering depends on local conditions such as local climate, surface and groundwater conditions, chemicals dissolved in groundwater, and land and fertilizer use (Saunders and Fookes, 1970; Fookes et al., 1988; Semhi et al., 2000). The rates with which the processes take place are also very dependent on local conditions such as temperature and presence of water (White and Blum, 1995; Bland and Rolls, 1998). 


\subsubsection{Weathering at surface versus subsurface}

Weathering processes in the subsurface are different from those in an exposure at surface. In the first situation weathering is mostly governed by chemical weathering whereas physical weathering is largely absent, while in exposures where weathering takes place under the direct influence of the atmosphere, it is a combination of chemical and physical weathering. In an exposure, biological agents may be of influence too (Hinsinger et al., 2001), and stress relief may be more pronounced at surface than in the subsurface. Many parameters are of influence on weathering rates in the subsurface such as the stress regime, the depth of the rock mass below surface, the hydrological regime (Day et al., 1980; Katongo, 2005), and chemicals dissolved in groundwater. These parameters may change over time. Backcalculating weathering rates in the subsurface are mostly done from mass balances of chemicals dissolved in groundwater (e.g. Moreira-Nordemann, 1980; White et al., 1998; Olvmo, 2010), although many studies include necessarily also weathering at surface, as it is mostly impossible to differentiate between chemicals derived from surface and subsurface weathering.

\subsubsection{Intact rock weathering}

Many studies have been done to the weathering of intact rock (Figure 3.1). Most concern ornamental stone, which mostly implies that the rock is freely exposed to weather influences. For example, many studies have been done on tomb- grave- or head-stones (among many studies: Dragovich, 1987; McNeill, 1999; Inkpen and Jackson, 2000; Williams and Robinson, 2000; Wells et al., 2008), natural rock blocks mounted on the outside of buildings (Warke et al., 2006; Ruiz-Agudo et al., 2007), or natural rock in and on monuments, such as Cleopatra's Needle in New York (Winkler, 1980; Storemyr, 2012), Medieval castles and churches in Spain (Sancho et al., 2003; Sebastián et al., 2008), and ancient temples and monuments in Asia (Lee et al., 2005; Siedel et al., 2010). The weathering effect on construction materials especially rock aggregates has been discussed in detail by Fookes et al. (1988). Rates for weathering vary widely depending on rock material and climatic environment. A decrease of weathering rate is observed by many authors when the weathered material ("residue") stays on the surface, and then protects the underlying material. If the weathered material is removed i.e. falls off by gravity or is flushed away by water, weathering starts again with a weathering rate comparable to the weathering rate of the original material. In the Mediterranean area, the rates of weathering for building stone depend strongly on the way the building stone is used, i.e. in how far the building stone is isolated from coastal i.e. marine, influence (Al-Agha, 2006). Measured weathering rates for similar types of intact rock can be a 
factor 3.5 times faster in a humid tropical area compared to a cool humid temperate environment (Day et al., 1980).

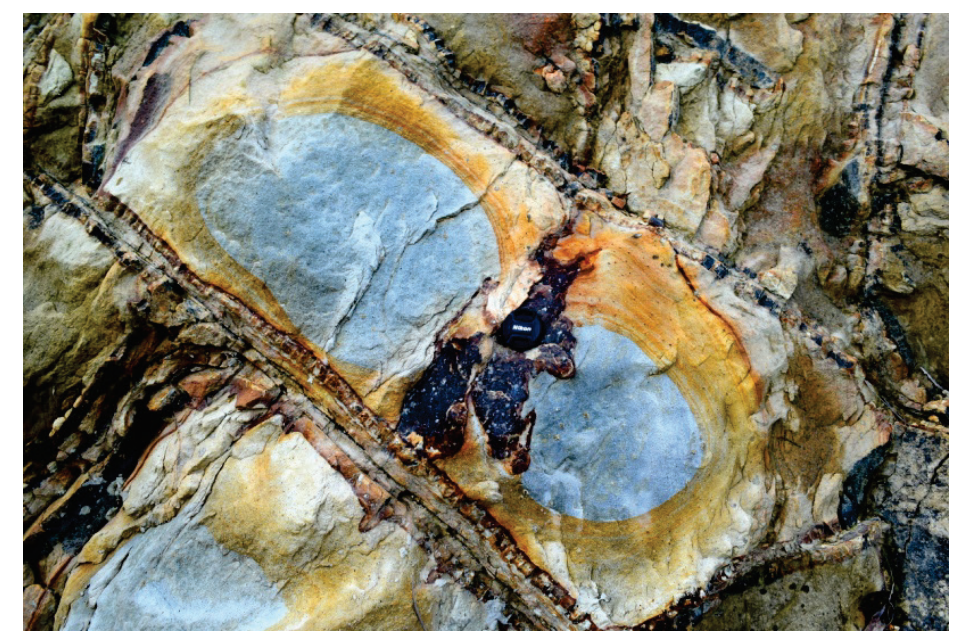

Figure 3.1 Weathering of intact rock. Note the formation of residual layer that will inhibit the weathering process towards the inner part of intact rock

\subsubsection{Rock mass weathering}

Natural rock masses contain intact rock but this rock is neither exposed to the atmosphere at all or only one site of a block if it is on the outside of an exposure. The influence of the atmosphere has to be transferred through other blocks or through discontinuities by water or air. This temporizes and moderates the effects of the outside environment on the inside of the rock mass. Deterioration in rock mass may effects the intact rock strength, spacing of discontinuities and the shear strength along discontinuities (Figure 3.2 and Figure 3.3). This is further discussed in chapter 4 and chapter 5 .

Rock mass weathering in tropical environments has been described for most intact rock and rock masses that occur in Malaysia, i.e. granite (Komoo, 1985; Raj, 1985; Ghiasi et al., 2009; Chigira et al., 2011; Mohamad et al., 2011), sandstone/shale (Mohamed et al., 2007; Mohamad et al., 2008), limestone (Pauzi et al., 2011) and schist (Zauyah and Stoops, 1990). Most of the studies are related to the weathering effect on the physical and geotechnical properties of rock (such as durability, modulus of elasticity, compressive strength), mineralogical changes, and characterization of the weathering profile. Generally, they conclude that weathering has a significant effect on the rock geotechnical and physical properties, and results in deep weathering profiles. Relations between weathering and time are not mentioned in these studies. Mohamed et al. (2007) compare physical deterioration differences between sandstone and shale, and Rodeano et al. 
(2006) and Ismail et al. (2009) describe engineering properties of fresh and weathered sandstone in the Kota Kinabalu area.

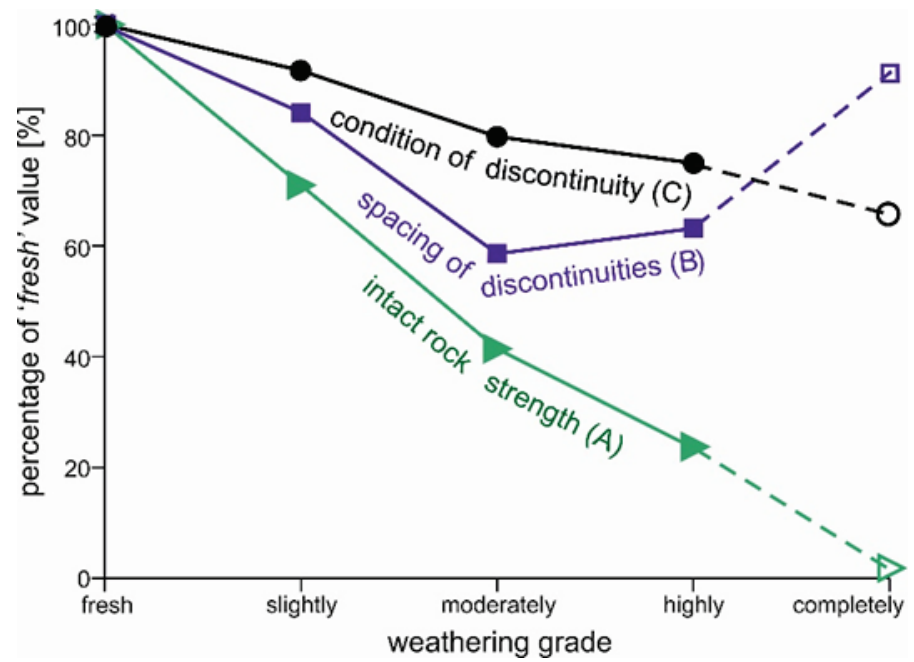

Notes: Data of various sources and rock masses; averaged after normalization with values for fresh equal $100 \%$. Standard deviation around 15 to $23 \%$ (points) for slightly through highly weathered; data for completely weathered are few and average not reliable. Fresh, slightly, etc. refer to rock mass weathering following ISO 14689-1;2003. "Spacing of discontinuities" based on rock block size and form following Taylor (1980) in Hack et al. (2003) or on discontinuity spacing 'Condition of discontinuity' (determining the shear strength) following sliding criterion (Hack et al., 2003) or friction and cohesion properties for discontinuities. Data: A: 1, 5, 6, 7 \& 10; B: 2, 3, 4 \& 5; C: 5,8 \& 9. (1) Begonha \& Sequeira Braga (2002); (2) Ehlen (1999); (3) Ehlen (2002); (4) GCO (1990); (5) Hack \& Price (1997); (6) Marques et al., (2010); (7) Pickles (2005) (8); Reissmüller (1997) (9); Snee (2008); (10) Tugrul (2004).

Figure 3.2 Influence of weathering and stress relief on intact rock and rock mass properties (after Hack, 2011).

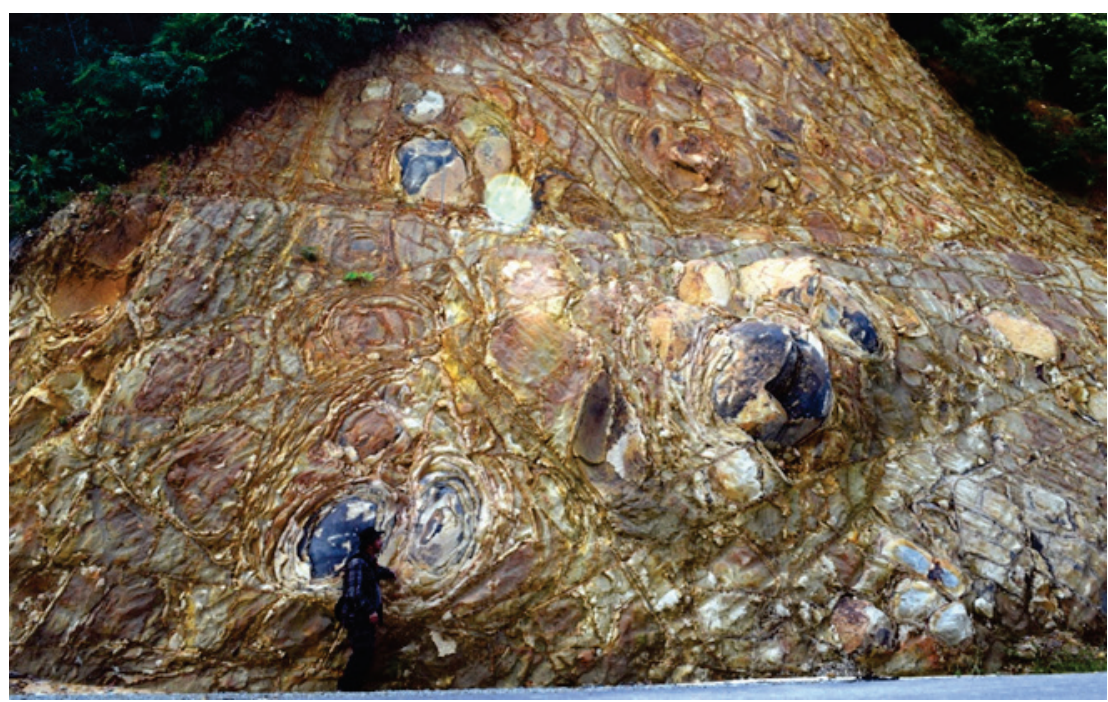

Figure 3.3 Weathering of rock mass. Weathering take place along the discontinuities and affected the discontinuity shear strength. 


\subsection{Rock mass weathering with time}

Rock mass weathering affects the durability of a rock slope after excavation (Hack and Price, 1997; Yokota and Iwamatsu, 2000; Hack et al., 2003; Borrelli et al., 2007; Hencher and Knipe, 2007). Weathering in man-made slopes is controlled by internal, external and geotechnical parameters (Bland and Rolls, 1998; Huisman, 2006). Internal parameters are referring to the properties of rock materials, whereas external parameters are the environmental conditions, fluid chemistry, hydrodynamics of the site and biogenic activities. Slope design parameters (e.g. slope aspect, angle and height), method of excavation and drainage measures also influence the degradation of geotechnical properties.

\subsubsection{Weathering intensity and weathering intensity rate}

The relationship between rock mass response to the process of weathering and exposure time is expressed in two different terms; i.e. "weathering intensity" and "weathering intensity rate". Weathering intensity refers to the degree of decomposition or amount of alteration from the original state shown by rock mass material at a certain point in time, whereas the weathering intensity rate refers to the amount of change in this weathering intensity per unit time (Bland and Rolls, 1998; Huisman, 2006). The weathering intensity and weathering intensity rate of a rock mass respond to the particular combination of conditions controlling weathering at the site of the exposure. This may be referred to as the susceptibility to future weathering of the rock mass (Hack, 1998). Moreover, the weathering intensity rate is dependent on whether the material already weathered ("residue") is removed or forms a cover protecting the underlying material (Colman, 1981), an effect that is similar to protecting intact rock by weathered material (see Section 3.2.2.2).

As mentioned before, the rock mass weathering in a man-made slope is controlled by various parameters. These parameters are also governing the intensity and rate of weathering. Both internal and external weathering controlling conditions are functions of time and may have considerably changed from conditions in the past during the history of the site. Geotechnical conditions may possibly change in the course of time by modification of the slope profile (Huisman, 2006). Intensity rate of weathering may also be affected by the degree of imbalance between weathering and erosion or other surface activities. Erosion and shallow slope failure processes remove the weathering product from the rock surface, which will expose a new fresh or partially weathered surface. Thus, once again increases the weathering intensity rate. This process will result in frequent failures in a man-made slope and tend to repeat over time (Yokota and Iwamatsu, 2000). 
Weathering intensity rates may be expected to decrease with time, as the state of rock mass becomes more and more in equilibrium with its surroundings (Huisman, 2006). Bland and Rolls (1998) have discussed several approaches for determining weathering intensity rates, whereas several publications (e.g. Hachinohe et al., 2000; Huisman et al., 2006; Nishiyama and Matsukura, 2009) report various methods to determine the rates of weathering in different rock types and climatic environments.

\subsubsection{Quantification of weathering in time}

Several methods are used to quantify weathering or weathering intensity rates. These methods may be divided into "uncontrolled" and "controlled" (Colman, 1981) based on the availability of time control and quantification of weathering variables. The "controlled" methods are further divided into three main groups (Fookes et al., 1988); "experimental", "man-made structures", and "geological materials group" based on the method used and the type of time control. Selection of the appropriate study method depends on the objective of and relevance for the object studied. For instance, for a manmade slope, the time control is within engineering times and it is controlled research as being a man-made structure.

\subsection{Rock and rock mass properties}

Various rock mass properties that are modified by weathering are used to determine the weathering intensity rates (e.g. Hachinohe et al., 2000; Yokota and Iwamatsu, 2000; Nishiyama and Matsukura, 2009). The common method is by determining the change brought by the processes of weathering to these properties. It is done by comparing the initial property value before and after weathering has occurred.

In a rock slope, the initial property value may be the value at the time of excavation whereas the weathered property value is the observed value at a certain time after the excavation (Irfan and Dearman, 1978; Selby, 1993; Hack and Price, 1997; Huisman, 2006). Irfan and Dearman (1978) used the ratio of unsound to sound constituents' of rock, which they defined as the Micropetrogaphic Index (Ip). Selby (1993) suggested using the ratio of unweathered to weathered minerals, defined as the Weathering Index (WI). Hack (1998) used the ratio of weathered to unweathered rock mass parameter (the weathering quantitative reduction value, WE) for quantifying the weathering intensity in the Slope Stability Classification System (SSPC). These SSPC weathering values (WE) are used by Huisman (2006) in his research on rock mass decay in geo-engineering. 


\subsubsection{Weathering intensity rate}

The weathering intensity rate can be determined if the exposure time for the rock mass is known. The time relation is corresponding to the expression proposed by Ruxton (1968) and Selby (1993):

$C_{t}=C_{t} e^{-k t}$

$C_{t}$ is the property value at time $t, C_{o}$ is the initial property value (original value in fresh state), $k$ is a constant, and $e$ is the base of the natural logarithm. An alternative empirical relationship to describe change of the property value as a function of time is suggested by Colman (1981):

$\frac{c_{t}}{c_{o}}=a+b \log (1+t)$

In which $a$ and $b$ are constants, $C_{t}$ refers to the parameter value at time $t$ and $C_{o}$ is the initial parameter value (original value in fresh state). The equation is also used by Huisman (2006) in describing the function of weathering intensity with exposure time $t$, by replacing the ratio $\frac{C_{t}}{c_{o}}$ with the weathering quantitative reduction value $\left(W E_{t}\right)$, constant $a$ with the $W E$ value at the time of excavation $\left(W E_{\text {init }}\right)$, and constant $b$ with the apparent weathering intensity rate $\left(R_{W E}^{a p p}\right)$ :

$W E_{t}=W E_{\text {init }}+R_{W E}^{a p p} \log (1+t)$

The same equation is used to describe the change of rock properties as function of time. The ratio $\frac{c_{t}}{c_{o}}$ corresponds to the property change at a certain time after the rock mass is excavated with respect to the initial value of the property. Property refers to the geotechnical properties such as intact rock strength (IRS), and internal angle of friction and cohesion of a rock mass. These properties are preferred as these are directly related to slope stability. The constant $a$ in Equation $\mathbf{3 . 2}$ is replaced by the initial property value $\left(I R S_{\text {init }}\right), b$ is replaced by the apparent rate of the change in $\operatorname{IRS}\left(R_{I R S}^{a p p}\right)$, and $\frac{C_{t}}{C_{o}}$ is replaced by $I R S_{t}$. The IRS value at exposure time $t$ becomes:

$I R S_{t}=I R S_{\text {init }}+R_{I R S}^{a p p} \log (1+t)$

The rate of change of the property value in this equation refers to the "apparent rate" which is quantified by the change in property value from the initial state divided by the total exposure time. The property value assessment made during a successive time series divided by the elapsed time between the assessments is the "dynamic rate" of the property value change at a particular time and location (Figure 3.4). 


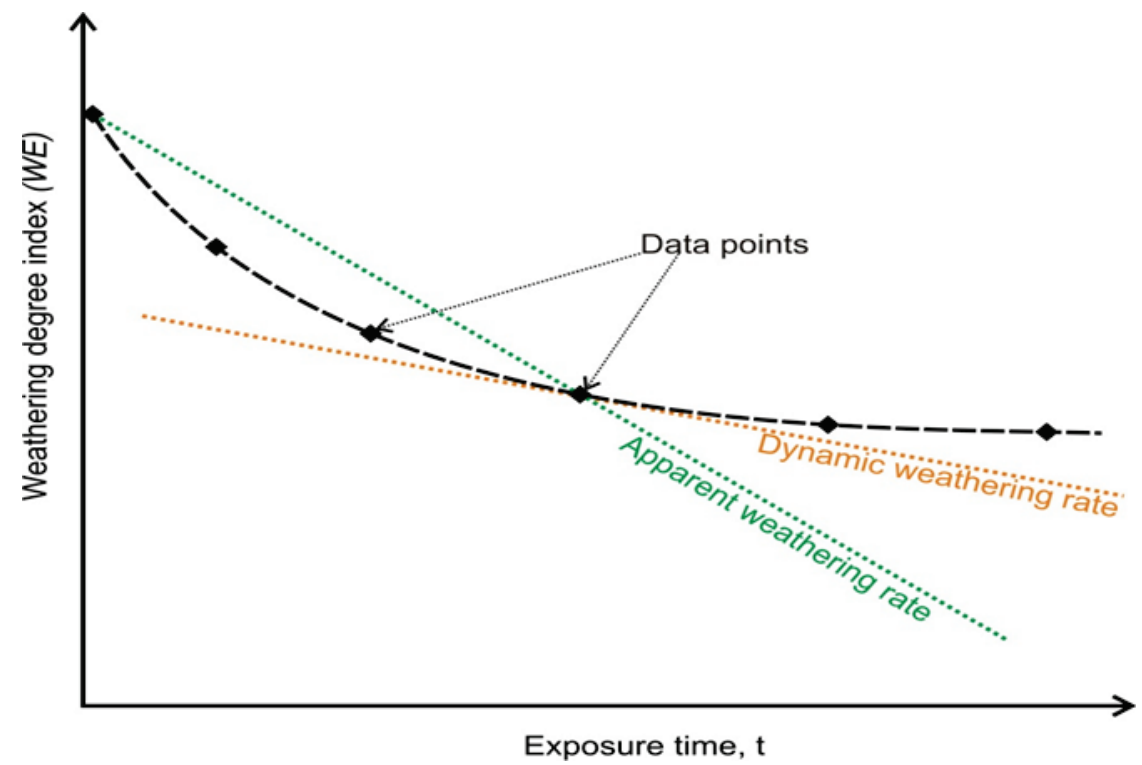

Figure 3.3 Definition of apparent and dynamic property change rates (Modified from Huisman, 2006).

Non-linearity of the weathering intensity rate has been attributed to the formation of a metastable protective residual layer (Colman, 1981; Ollier, 1984; Fookes et al., 1988) on an outer layer of fresh rock. The residual layer will impede the direct contact between the fresh rock material and the external weathering agents, such as rain, wind, humidity, and temperature. As the relationship between weathering and exposure time is expressed as property change at a certain time after the rock is excavated with respect to the initial value of the property, the delay in direct contact will decrease the weathering intensity rate. The thickness of this weathered material also increases with time (Hachinohe et al., 2000) and will further decrease the rate. If the residue layer is removed by any surface activities (e.g. slope failure or washed off by rain), a new cycle of weathering will start again.

\subsection{Methodology}

The main weathering agencies from the atmosphere affect the rock mass from the outside in a newly excavated exposure. The rocks on the outside of the exposure are first affected and later the rock mass deeper below surface will be affected (see Section 3.2 .2 .3 ). In this study, the rock mass in an exposure is divided in geotechnical units (see Section 1.6.1). Only the unit directly adjacent to the exposure face is used in this study. This guarantees that the exposure time is from the first onset of weathering after excavation until the moment of the field campaign. 
The grade of rock mass weathering is determined visually following Table 3.1 which is largely similar to the methodology described in BS 5930 (1981) and ISO 14689-1 (2003). IRS is estimated following Table 3.2, which is based on the IRS estimation procedure in BS 5930 (1981) and BS 5930 (1999). This procedure is largely comparable to the procedure in ISO 146891 (2003), but with different boundaries and slightly different descriptions. The methodology allows for estimating the intact rock strength by hand and geological hammer in the field. The methodology is also known as 'simple means' field test and has proven to be very effective in estimating representative values for the IRS (Hack and Huisman, 2002). At least five assessments of the IRS have been made for each unit. In addition and as verification, samples have been tested with the " $L$ " type Schmidt Hammer $(\mathrm{SH})$.

Table 3.1 Rock mass weathering grades

\begin{tabular}{|c|c|c|c|c|}
\hline Grade $^{a, b}$ & Grade $^{c}$ & Term $^{\text {b }}$ & Description $^{b}$ & $W E^{a}$ \\
\hline $\mathrm{I}$ & 0 & Fresh & $\begin{array}{l}\text { No visible sign of rock material } \\
\text { weathering; perhaps slight } \\
\text { discoloration on major } \\
\text { discontinuity surfaces }\end{array}$ & 1.00 \\
\hline II & 1 & Slightly weathered & $\begin{array}{l}\text { Discoloration indicates } \\
\text { weathering of rock material and } \\
\text { discontinuity surfaces. }\end{array}$ & 0.95 \\
\hline III & 2 & $\begin{array}{l}\text { Moderately } \\
\text { weathered }\end{array}$ & $\begin{array}{l}\text { Less than half of the rock } \\
\text { material is decomposed or } \\
\text { disintegrated. }\end{array}$ & 0.90 \\
\hline IV & 3 & Highly weathered & $\begin{array}{l}\text { More than half of the rock is } \\
\text { present either as a discontinuous } \\
\text { framework or as core stones. }\end{array}$ & 0.62 \\
\hline V & 4 & $\begin{array}{l}\text { Completely } \\
\text { weathered }\end{array}$ & $\begin{array}{l}\text { All rock material is decomposed } \\
\text { and/or disintegrated to soil. The } \\
\text { original mass structure is still } \\
\text { largely intact. }\end{array}$ & 0.35 \\
\hline VI & 5 & Residual soil & $\begin{array}{l}\text { All rock material is converted to } \\
\text { soil. The mass structure and } \\
\text { material fabric are destroyed. } \\
\text { There is a large change in } \\
\text { volume, but the soil has not } \\
\text { been significantly transported }\end{array}$ & - \\
\hline
\end{tabular}

Notes: a) From Hack et al. (2003); b) Based on BS 5930 (1981); and c) ISO

14689-1 (2003) 
Table 3.2 Field determination of intact rock strength (IRS) by "simple means" test. Modified from BS 5930:1981; BS 5930: 1999

\begin{tabular}{|c|c|c|}
\hline $\begin{array}{l}\text { IRS } \\
(\mathrm{MPa})\end{array}$ & Field Identification & Term \\
\hline$<1.25$ & $\begin{array}{l}\text { Gravel size lumps can be crushed between finger } \\
\text { and thumb }\end{array}$ & Very weak \\
\hline 1.25 to 5 & $\begin{array}{l}\text { Gravel size lumps can be broken half by heavy } \\
\text { hand pressure or thin slabs break easily in hand }\end{array}$ & Weak \\
\hline 5 to 12.5 & $\begin{array}{l}\text { Only thin slabs, corners or edges can be broken off } \\
\text { with heavy hand pressure }\end{array}$ & Moderately weak \\
\hline $\begin{array}{l}12.5 \text { to } \\
50\end{array}$ & $\begin{array}{l}\text { When held in hand, lumps can be broken by light } \\
\text { hammer blow }\end{array}$ & Moderately strong \\
\hline $\begin{array}{l}50 \text { to } \\
100\end{array}$ & $\begin{array}{l}\text { When resting on a solid surface, lumps can be } \\
\text { broken by heavy hammer blows }\end{array}$ & Strong \\
\hline $\begin{array}{l}100 \text { to } \\
200\end{array}$ & Lumps only chipped by heavy hammer blows & Very strong \\
\hline$>200$ & $\begin{array}{l}\text { Rocks ring on hammer blows. Spark fly. Only } \\
\text { broken by sledgehammer. }\end{array}$ & Extremely strong \\
\hline
\end{tabular}

\subsubsection{Rock mass cohesion and friction angle estimation}

Other rock geotechnical parameters such as cohesion and friction angle values are estimated by optimizing the Mohr-Coulomb failure criterion with intact rock strength (IRS), discontinuity spacing (SPA) and condition of discontinuity (CD) in the SSPC system (Hack et al., 2003). The equations are given by:

$$
\begin{aligned}
& \text { Friction }_{\text {mass }}=0.2417 I R S+52.12 S P A+5.779 C D \\
& \text { Cohesion }_{\text {mass }}=94.271 I R S+28629 S P A+3593 C D
\end{aligned}
$$

Frcition $_{\text {mass }}$ is the angle of internal friction angle of rock mass (in degrees) and Cohesion $_{\text {mass }}$ is the rock mass cohesion (in Pascal).

\subsubsection{Exposure time}

The time the exposure exists is required for establishing the relationship between weathering and time. The date of excavation is obtained from the agencies or operators responsible for the excavation, e.g. the Public Works Department of Malaysia (PWD) for most roadcuts and quarry operators for rock quarries. For privately excavated slopes the excavation dates has been obtained by enquiring the local residents residing in the vicinity of the excavations. Some exposures in the area have been eroded, re-excavated, or otherwise the rock mass forming the slope face at present is not anymore the same as exposed directly after excavation. Indications that something has happened with the rock mass in an exposure after first excavation are, for example, considerable quantities of material fallen from the exposure, (recent) fresh marks from excavation equipment, marks from excavation equipment differently from the equipment used for the first excavation, signs 
of erosion in the exposure, and erosion above or below the exposure more recent than could be expected from the geomorphological setting before first excavation. The exposure time for the rock mass in such exposures is then not anymore representing the time from the onset of weathering after first excavation and therefore these exposures were disregarded for this research.

\subsubsection{Fresh rock}

In tropical areas, it is often difficult to find rock masses not weathered (i.e. fresh) because rock masses weather very rapidly and the weathering profile is very thick. The latter causes that the fresh rock is often not exposed in excavations because excavations are not deep enough horizontally nor vertically to expose the fresh rock mass. However, in the study area, the extensive new housing and infrastructure development requires many new excavations in and through the hills. The excavation of fresh rock mass by conventional excavation machines is difficult and blasting is often prohibited. The hill is then excavated until excavation becomes too cumbersome, which is often at a point where the bulk of the rock mass becomes un-weathered.

This fresh rock mass can be examined. In addition, fresh rock mass (i.e. fresh at the time of the fieldwork campaigns for this research) is exposed in quarries made for building and construction material within or in the vicinity of the study area. Validating that a rock mass encountered in an exposure is fresh is done visually, similar to the characterization of the weathered rock masses. Signs of weathering are evaluated visually and then the grade of rock mass weathering is established following BS 5930 (1981) and ISO 14689-1 (2003).

\subsubsection{Weathering before and after excavation}

The degree of rock mass weathering in the exposure directly after excavation differs. Some exposures show weathered rock mass that is thus already weathered before excavation, others show fresh rock mass. The exposure times for rock masses that are already weathered at the time of excavation do not refer to the onset of weathering of the non-excavated rock mass. The latter is impossible to obtain because the date of the onset of weathering is not known. Only for exposures that show the rock mass in fresh state at the time of excavation, the exposure time is the same as the time the rock mass is exposed to the processes of weathering in the exposure. 


\subsection{Results}

\subsubsection{Correlation of intact rock strength determined by testing and "simple means"}

The sedimentary strata in the study area are divided into three lithological units: i) the thick to very thick-bedded sandstone (SST), ii) a thinly interbedded unit with sandstone, siltstone, and shale (IB) layers and iii) thick bands of red or grey shale (SH) (see Table 2.1, Chapter 2).

In previous studies, representative rock samples from each rock mass weathering grade have been collected and tested in the laboratory to determine the intact rock strength (IRS) by Unconfined Compressive Strength (UCS) and Point Load Strength (PLS) tests (Table 3.3). The IRS of fresh sandstone of the SST unit based on UCS tests ranges from 88 to 130 $\mathrm{MPa}$, and the average IRS estimated by "simple means" is $105 \mathrm{MPa}$. The correlation between the UCS tests and "simple means" test values per rock mass weathering grade (Table 3.2) is shown in Figure 3.5.

In this article, only the thick to very thick-bedded sandstone unit (SST) is studied. The other two units are continuously eroding, exposing new fresh rock, which makes it difficult to determine the effect of weathering on the rock properties with time. The summary of the SST unit is shown in Table 3.4. 
Table 3.3 Point Load Strength (PLS) and Unconfined Compressive Strength (UCS) for sandstone of the Crocker Formation.

\begin{tabular}{|c|c|c|c|c|}
\hline \multirow[t]{2}{*}{ Sample } & \multirow{2}{*}{$\begin{array}{c}\text { Rock mass } \\
\text { weathering } \\
\text { grade }\end{array}$} & \multicolumn{2}{|r|}{$\begin{array}{c}I R S \\
(\mathrm{MPa})\end{array}$} & \multirow{2}{*}{$\begin{array}{l}\text { Density } \\
(\mathrm{kg} / \mathrm{m3})\end{array}$} \\
\hline & & PLS & UCS & \\
\hline & III & - & 15.5 & 2066 \\
\hline $\mathrm{BH} 8(\mathrm{C3})^{\mathrm{a}}$ & III & 1 & - & - \\
\hline $\mathrm{BH} 4(\mathrm{C5})^{\mathrm{a}}$ & IV & 0.04 & - & - \\
\hline $\mathrm{BH} 1(\mathrm{C} 3)^{\mathrm{a}}$ & IV & 0.52 & - & - \\
\hline \multicolumn{5}{|l|}{ BH4(C1) } \\
\hline BH4(C13) & II & - & 63.7 & 2791 \\
\hline $\mathrm{BH} 4(\mathrm{C16})^{\mathrm{b}}$ & II & - & 50.9 & 2736 \\
\hline BH5(C9) ${ }^{b}$ & II & - & 61.1 & 2787 \\
\hline BH5(C11) & II & - & 38.2 & 2694 \\
\hline BH5(C13) & II & - & 45.8 & 2601 \\
\hline $\mathrm{BH} 4(\mathrm{C7})^{\mathrm{b}}$ & III & - & 22.9 & 2542 \\
\hline $\mathrm{BH} 5(\mathrm{C7})^{\mathrm{b}}$ & III & - & 28 & 2610 \\
\hline BH4 $(\mathrm{C} 4)^{\mathrm{b}}$ & IV & - & 7.6 & 2467 \\
\hline BH5(C5) & IV & - & 7.6 & 2380 \\
\hline 12 Samples $^{c}$ & III - V & $0.44^{1}$ & $10.5^{1}$ & na \\
\hline 179 Samples $^{d}$ & I & - & $123^{1}-130^{2}$ & - \\
\hline G3(C1) & I & - & 88.1 & 2607 \\
\hline G3(C2) & I & - & 99.3 & 2458 \\
\hline G3 $(\text { C3 })^{e}$ & I & - & 88.1 & 2479 \\
\hline G2 $(C 2)^{e}$ & III & - & 12.1 & 2420 \\
\hline $\mathbf{G 2}\left(\mathrm{C}_{3}\right)^{\mathrm{e}}$ & III & - & 21 & 2445 \\
\hline$K K 43^{f}$ & I & - & 100 & - \\
\hline$K K 65^{f}$ & I & - & 105 & 2649 \\
\hline$K K 70^{f}$ & II & - & 90 & - \\
\hline$K K 78^{f}$ & II & - & 70 & 2495 \\
\hline$K K 5^{f}$ & II & - & 40 & - \\
\hline$K K 52^{f}$ & II & - & 70 & - \\
\hline$K K 15^{f}$ & III & - & 20 & - \\
\hline$K K 8^{f}$ & III & - & 30 & 2350 \\
\hline$K K 11^{f}$ & III & - & 40 & - \\
\hline
\end{tabular}


Table 3.4 IRS values for thick to very thick-bedded sandstone (SST) unit.

\begin{tabular}{|c|c|c|c|c|c|}
\hline \multirow{2}{*}{$\begin{array}{l}\text { Slope } \\
\text { no. }\end{array}$} & \multirow{2}{*}{$\begin{array}{c}\text { Rock mass } \\
\text { weathering }_{\text {grade }^{\mathrm{a}}}\end{array}$} & \multirow{2}{*}{$\begin{array}{l}\text { Observed IRS } \\
\quad(\mathrm{MPa})\end{array}$} & \multirow{2}{*}{$\begin{array}{c}\text { Exposure } \\
\text { time } \\
\text { (year) }\end{array}$} & \multicolumn{2}{|c|}{$\begin{array}{c}\text { Adjusted exposure time } \\
\text { (year) }\end{array}$} \\
\hline & & & & Logarithmic $^{d}$ & Exponential $^{\mathrm{e}}$ \\
\hline KK2 & IV & 10 & 7 & 622 & 70 \\
\hline KK4 & IV & 4 & 13 & 935 & 97 \\
\hline KK5 & II & 40 & 26 & 26 & 26 \\
\hline KK6 & IV & 4 & 26 & 935 & 97 \\
\hline KK7 & III & 25 & 15 & 225 & 43 \\
\hline KK8 & III & 30 & 35 & 35 & 35 \\
\hline KK11 & III & 40 & 15 & 82 & 29 \\
\hline KK14 & IV & 5 & 15 & 873 & 91 \\
\hline KK15 & III & 20 & 15 & 316 & 50 \\
\hline KK16 & III & 30 & 15 & 161 & 38 \\
\hline KK17 & IV & 14 & 15 & 475 & 60 \\
\hline KK19 & IV & 1.5 & 13 & 1107 & 126 \\
\hline KK23 & IV & 10 & 0.5 & 622 & 70 \\
\hline KK25 & IV & 6 & 15 & 816 & 85 \\
\hline KK27 & IV & 3 & 15 & 1000 & 106 \\
\hline KK29 & III & 14 & 7 & 475 & 60 \\
\hline KK30 & IV & 6 & 7 & 816 & 85 \\
\hline KK31 & IV & 14 & 15 & 475 & 60 \\
\hline KK36 & III & 30 & 17 & 161 & 38 \\
\hline KK38 & III & 13 & 17 & 508 & 62 \\
\hline KK39 & II & 75 & 7 & 7 & 7 \\
\hline KK40 & IV & 13 & 7 & 508 & 62 \\
\hline KK43 & I & 100 & 1 & 1 & 2 \\
\hline KK45 & II & 45 & 15 & 58 & 26 \\
\hline KK48 & III & 18 & 15 & 362 & 53 \\
\hline KK50 & III & 16 & 15 & 415 & 56 \\
\hline KK51 & III & 16 & 15 & 415 & 56 \\
\hline KK52 & II & 70 & 15 & 15 & 15 \\
\hline KK53 & II & 65 & 15 & 15 & 15 \\
\hline KK54 & II & 70 & 15 & 15 & 15 \\
\hline KK57 & III & 40 & 15 & 82 & 29 \\
\hline KK59 & IV & 10 & 0.1 & 622 & 70 \\
\hline KK60 & V & 2 & 0.1 & 1070 & 118 \\
\hline KK61 & IV & 14 & 2 & 475 & 60 \\
\hline KK64 & V & 4 & 2 & 935 & 97 \\
\hline KK65 & I & 105 & 1 & 1 & 1 \\
\hline KK70 & II & 90 & 5 & 5 & 5 \\
\hline KK71 & IV & 12 & 15 & 544 & 65 \\
\hline KK75 & IV & 7 & 15 & 763 & 81 \\
\hline KK77 & IV & 14 & 15 & 475 & 60 \\
\hline KK78 & II & 70 & 17 & 17 & 17 \\
\hline KK79 & IV & 10 & 1 & 622 & 70 \\
\hline KK82 & III & 16 & 1 & 415 & 56 \\
\hline KK83 & I & 105 & 1 & 1 & 1 \\
\hline KK85 & IV & 6 & 18 & 816 & 85 \\
\hline KK86 & II & 20 & 15 & 316 & 50 \\
\hline KK87 & IV & 12 & 15 & 544 & 65 \\
\hline KK88 & III & 25 & 1.5 & 225 & 43 \\
\hline KK91 & IV & 6 & 1.5 & 816 & 85 \\
\hline KK92 & III & 16 & 1.5 & 415 & 56 \\
\hline КК93 & IV & 12 & 1.5 & 544 & 65 \\
\hline KK94 & III & 12 & 1.5 & 544 & 65 \\
\hline
\end{tabular}

aBased on BS 5930:1981 (1981), b IRS estimated following Table 5, c Time from dated of excavation, d Calculated from logarithmic function (Equation 5), e Calculated from exponential function (Equation 6) 


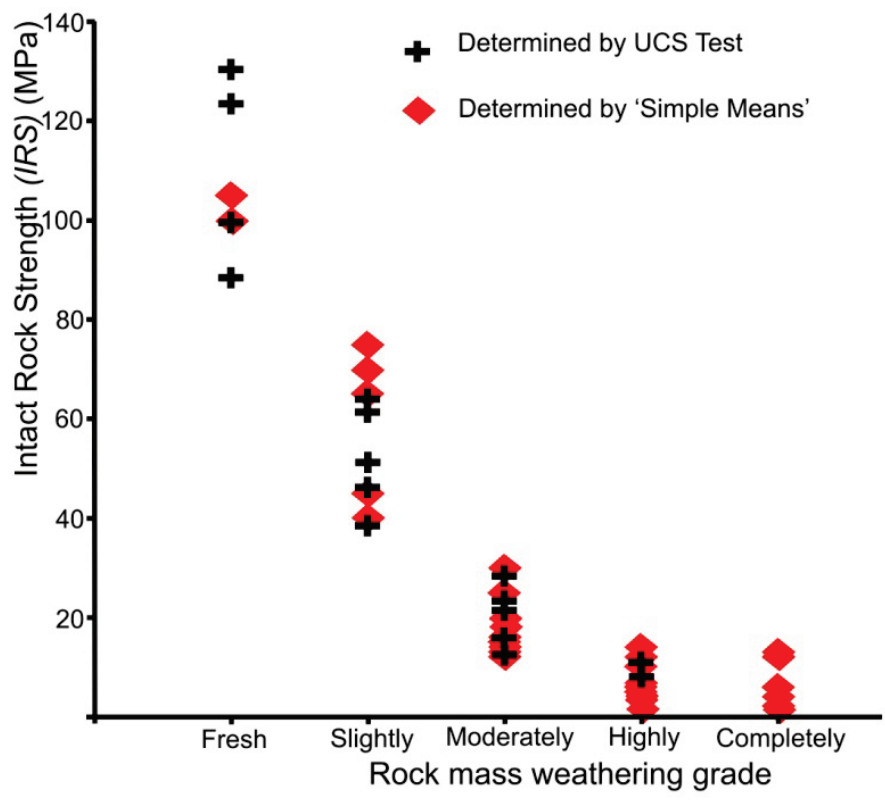

Figure 3.4 Comparison between unconfined Compressive Strength (UCS) tests (from various reports, Table 3.3) and "simple means" tests (Table 3.2) per rock mass weathering grade (Table $\mathbf{3 . 1}$ ).

\subsubsection{SST unit IRS and exposure time relation}

The relationship between the IRS and exposure time in the SST unit is shown in Figure 3.6. It shows that the IRS of the SST unit decreases non-linearly with time. This weathering effect can be expressed in both logarithmic (Figure 3.6a) and exponential functions (Figure 3.6b). The best fit by least squares is a logarithmic function:

$I R S_{t}=105+34 \log (1+t)$

$I R S_{t}$ (in $\mathrm{MPa}$ ) is the intact rock strength of sandstone at the time $(t), t$ is the time since exposure in years. The constant 105 is the initial value of the IRS in MPa of the fresh SST unit at the time of excavation (i.e. at exposure time 0 year), and 34 (in $\mathrm{MPa} / \log$ [year]) is the apparent reduction rate for this type of rock mass in the environment of the study area. This initial IRS value for the SST unit determined by this function is within the range of IRS of the fresh SST unit as determined in various studies before (i.e. in the range from 88 to $130 \mathrm{MPa}$, Table 3.3). In addition, an exponential function is fitted by least squares:

$I R S_{t}=108.7 e^{-0.034 t}$

$I R S_{t}$ (in $\mathrm{MPa}$ ) is the intact rock strength of sandstone at the time $(t), t$ is the time since exposure in years. The IRS of the SST unit in a fresh rock mass 
estimated with the exponential function is $108.7 \mathrm{MPa}$, which is within the range of the IRS for the fresh SST unit (Table 3.3). The first order constant rate for apparent IRS reduction rate of the SST unit is about $3.4 \times 10^{-2}$ $\ln /[$ year] .
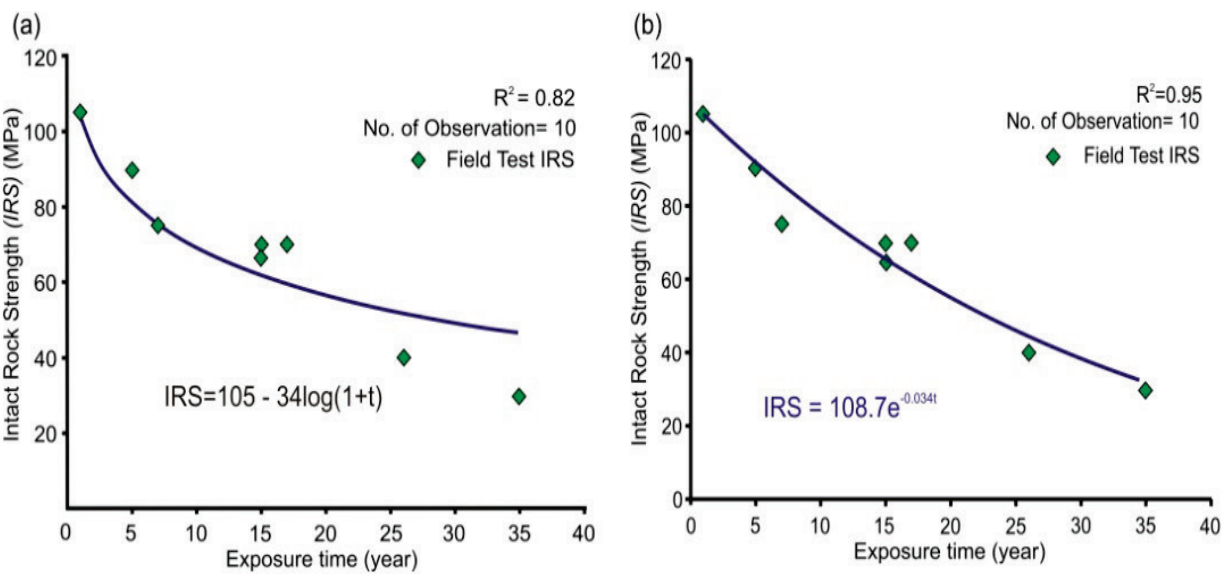

Figure 3.5 IRS vs. exposure time of SST unit; a) logarithmic function; b) exponential function.

\subsubsection{Adjusted exposure time}

The time from the onset of weathering of rock masses that were already weathered when excavated is the "adjusted exposure time" and determined by Equations (3.7) and (3.8). Obviously, back-calculating subsurface weathering with a relation obtained from weathering at surface is highly tentative and likely not completely correct. Weathering processes in the subsurface are different from those in open exposure (see Section 3.2.2.1). Subsurface weathering rates for longer time spans are generally unknown, and therefore, the adjusted exposure time back calculation is included because it gives an idea of how long the weathering would have taken if it had occurred in an exposure. This adjusted exposure time could probably and speculatively be regarded as a minimum weathering time.

The "adjusted exposure time" calculated with Equations (3.7) and (3.8) functions show a marked difference between the time dependency of IRS on weathering (Table $\mathbf{3 . 4}$ and Figure 3.7). Both functions show a high correlation $\left(R^{2}=0.82\right.$ for logarithmic and $R^{2}=0.95$ for exponential, Figure 3.6) hence this does not differentiate on validity and both may be applied in predicting the IRS deterioration of the SST unit (Figure 3.7). However, a logarithmic function is normally used to describe changes of rock properties over time (Colman, 1981; Bland and Rolls, 1998; Huisman, 2006) and therefore also used here. 
Equation (3.4) can be converted to define the apparent rate for the reduction of rock properties:

$R_{I R S}^{a p p}=\frac{I R S_{\text {initial }}-I R S_{\text {observed }}}{\log (1+t)}$

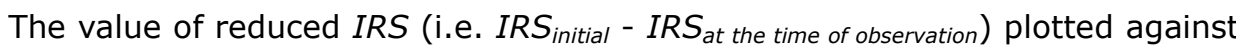
the logarithm of the exposure time shows a linear relation (Figure 3.7). This relation can be used to predict the reduction of IRS for the SST unit with time in the Kota Kinabalu area. The linear relation is given by:

$I R S_{\text {reduction }}=34 t+0.36$

The reduced IRS is equal to the IRS value (MPa) difference between fresh and weathered SST at certain time, whereas $t$ is log of the exposure time.

(a)

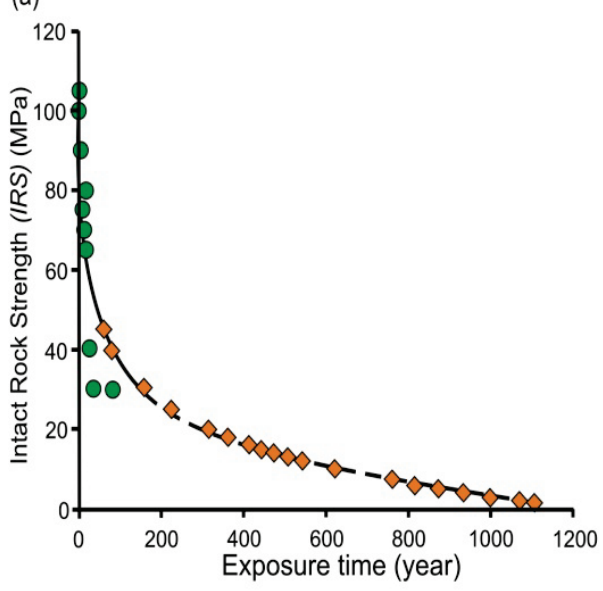

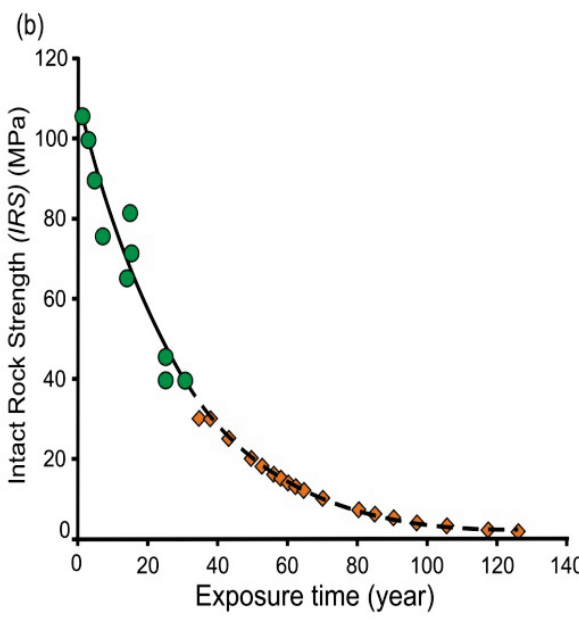

Observed IRS with estimated exposure time

Figure 3.6 Observed IRS vs. estimated exposure time of SST unit; (a) logarithmic function; (b) exponential function. 


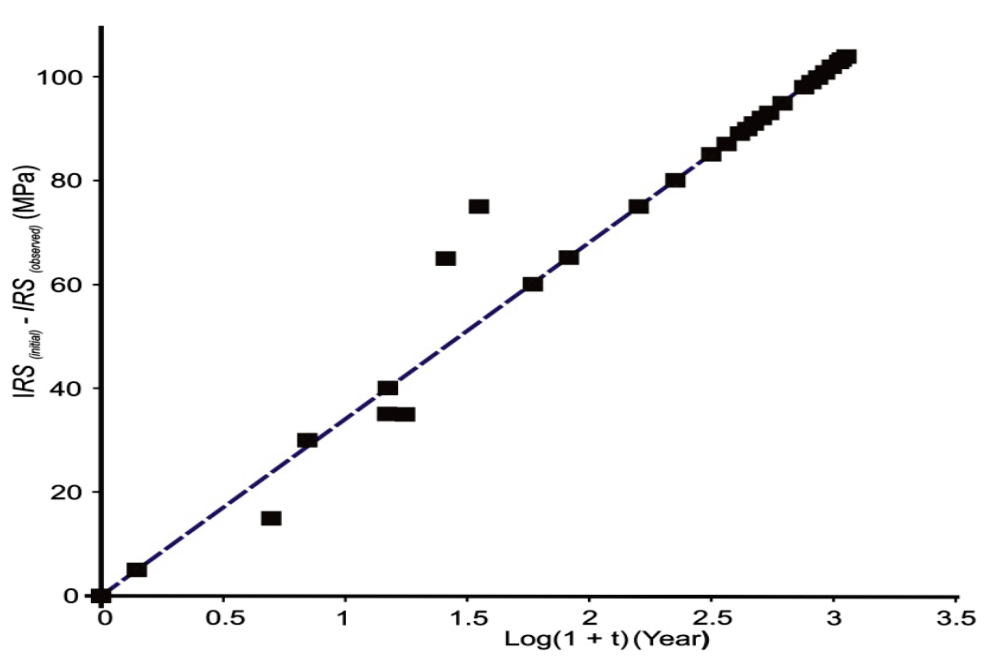

Figure 3.7 Linear relation of IRS vs. logarithmic estimated exposure time.

\subsubsection{Correlation between cohesion and friction angle of SST unit and exposure time}

Similar analyses are carried out for the cohesion and friction angle of the SST unit, which is estimated by using SSPC method (see Section 3.5.1). This is shown in Figure 3.9 and 3.10, respectively.

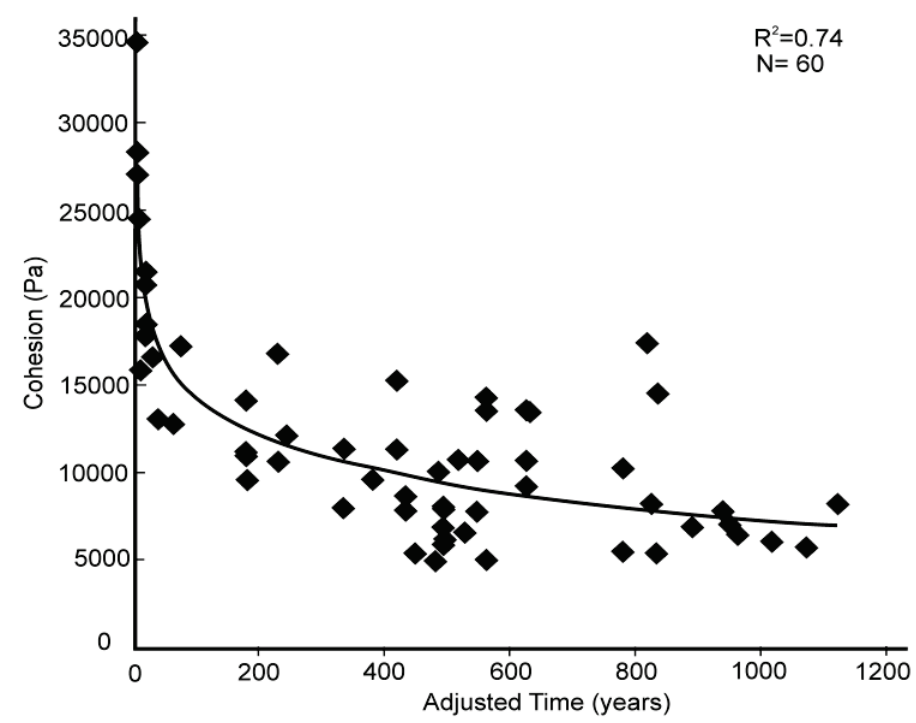

Figure 3.8 Estimated cohesion vs. estimated exposure time of SST Unit.

Based on the graphs (Figure 3.9), the SST unit cohesion reduction in time is expressed as:

Cohesion $_{t}=27004-6850.3 \log (1+t)$ 
Cohesion $_{t}(\mathrm{~Pa})$ is the intact rock cohesion of SST at the time $t$, and $t$ is the time since exposure in years. 27004 is the initial value of the cohesion in Pascal of the fresh SST unit at the time of excavation, and 6850.3 is the apparent reduction rate in $\mathrm{Pa} / \mathrm{log}$ [year]. For the SST unit friction angle (Figure 3.10), the relationship is expressed as:

Friction $_{t}=56.1-15 \log (1+t)$

Friction $_{t}$ (Deg) is the intact rock friction angle of SST unit at the time $t$, and $t$ is the time since exposure in years. 56.1 is the initial value of the cohesion in degree of the fresh SST unit at the time of excavation, and 153 is the apparent reduction rate in Degree/log [year].

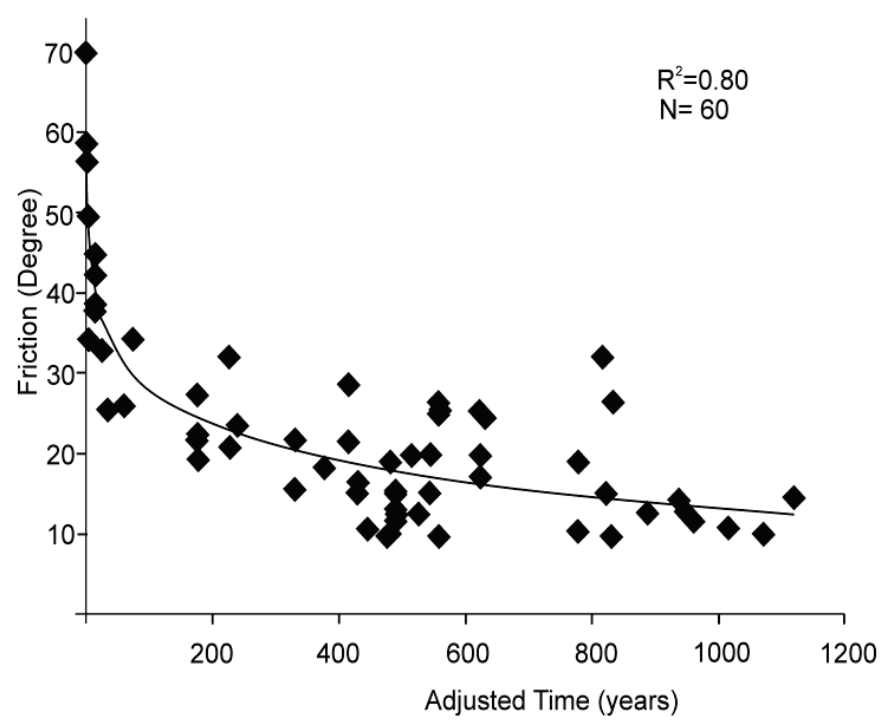

Figure 3.9 Estimated friction angle vs. estimated exposure time of SST Unit.

\subsection{Discussion}

The quantitative factors for the design of slopes in the thick to very-thick bedded sandstone (SST) unit to account for stress relief and weathering after excavation have been established for the Kota Kinabalu area. The relationship is expressed as a logarithmic function (Equation 3.7). The change of the IRS value with time due to weathering is non-linear as expected based on the literature. Figure $\mathbf{3 . 6}$ and Figure 3.7, show that the IRS change rate reduces after a certain period (noticeably after 50 years of exposure) and eventually becomes constant when the rock mass is transformed into a soil mass. Similar pattern are shown by SST unit cohesion and friction angle results (Figure $\mathbf{3 . 9}$ and Figure 3.10). The decrease of IRS change rate is explained by the formation of a protective residue, which prevents the 
weathering agent from direct contact with the fresh rock. The decrease of the IRS due to weathering applies to the outside of the slope where the rock mass is in contact with the atmosphere. For rock masses deeper under the surface, the IRS is likely also to decrease but at a smaller rate as the rock mass is protected from weathering by the mass above.

In order to correlate and compare with other studies the residual strength ratio (as percentage) is used as defined by Hachinohe et al. (2000):

$\%$ Residual Strength Ratio $=\frac{I R S_{t}}{I R S_{\text {init }}} * 100$

$I R S_{t}$ (in $\mathrm{MPa}$ ) is the measured intact rock strength at the time of observation, whereas $I R S_{\text {init }}$ (in $\mathrm{MPa}$ ) is the average intact rock strength of the fresh sandstone. Figure 3.11a shows the comparison between the results of this study and those obtained by Hachinohe et al. (2000). Both studies result in quite similar trends of residual strength ratio change over time. Both show a higher change rate of the residual strength ratio directly after exposure of the rock mass that gradually decreases with time. The change rates are different which is likely due to differences in lithological physical properties of the rock mass and in the climatic environment in which the studies were performed. Hachinohe et al. (2000) used a needle penetrometer to establish the relationship between strength ratio and time of two different sedimentary (sandstone and mudstone) rocks in a humid temperate climate in Japan.

Correlation and comparison with the study by Huisman (2006) (Figure 3.11b) is more difficult due to differences in rock mass parameters and methods used, and lithology and climatic environment differences. Huisman (2006) used the weathering quantitative reduction values (WE) to predict the weathering rate of different sedimentary rock masses in a Mediterranean climate in Spain. However, Huisman (2006) also show the non-linearity of property change in time due to weathering.

Both logarithmic and exponential functions show rapid changes of the IRS at the onset of stress relief and weathering after excavation. This rapid reduction corresponds to the response of the rock mass to the removal of overburden stress and the effect of weathering agents on the newly exposed fresh rock mass. Overburden removal causes changes of vertical and horizontal stress, isostatic adjustment, and temperature changes (Bland and Rolls, 1998). This will lead to outward displacement and contribute to the formation of micro-cracks. Development of sheeting joints in steep slopes may also be related to these processes (Hencher and Knipe, 2007; Hencher et al., 2011), which subsequently increases the rock mass porosity and permeability. The porosity and permeability changes will influence the conductivity, circulation and flow path of weathering agents such as water 
(Bland and Rolls, 1998), and thus, increase both the potential for chemical and physical weathering of the rock slope.

(a)

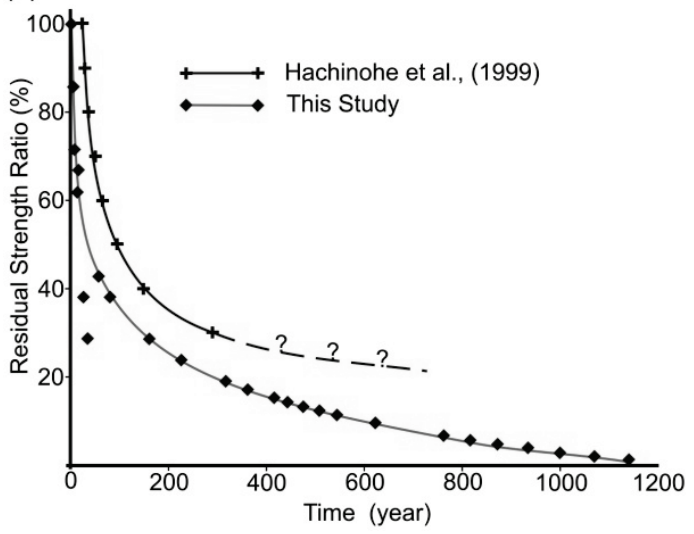

(b)

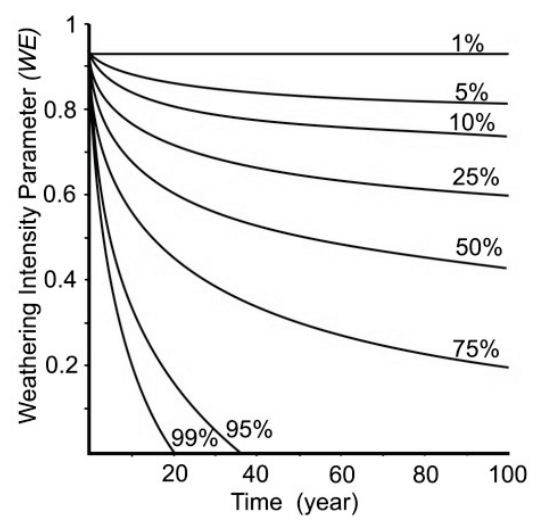

Figure 3.10 Comparison with other similar studies on weathering over time; (a) Hachinohe et al. (2000) in sandstone at depth of $0.01 \mathrm{~m}$ below surface; (b) weathering intensity parameter (WE) over time (modified from Huisman, 2006).

The reliability of the prediction of weathering with time depends on many site and location specific factors as discussed before. Thus, the prediction may only be applicable in an area where similar factors apply. All the weathering processes (i.e. chemical, physical, and biological processes) and stress relief are assumed to affect the IRS, cohesion and friction angle of SST unit simultaneously. Also it is assumed that the surface erosion of the rock mass (i.e. thick to very-thick bedded sandstone) is slower than the weathering processes.

Many of the intact rock strength (IRS) values were determined by "simple means" field tests (Hack and Huisman, 2002). It can be argued that the IRS resulting from these tests is subject to bias and depends on the person doing the tests. The authors do not think this to be an issue as the high number of tests carried out by various persons, and the results of control tests by Schmidt Hammer and previously done laboratory testing show consistency (Figure 3.2).

\subsection{Conclusion}

In this research, the relationship of the reduction of intact rock strength (IRS), cohesion and friction angle for thick to very-thick bedded sandstone with exposure time in the rock mass has been established for the SST unit of the Crocker formation in Sabah, Malaysia. The relation allows prediction of intact rock strength, cohesion and friction angle reduction within the "serviceable lifetime" of the slopes made in the SST unit, and can be used for 
better designing man-made slopes in the area. The weathering-time relation is likely also applicable for man-made slopes in other humid tropical areas, where similar weathering processes are the main reason for degradation of the rock mass after excavation. It should be noted that the weathering processes of the rock mass in the face of an exposure are different from those deeper below the surface. The rates for weathering of the deeper rock masses are likely lower than those presented in this article as the rock mass is more protected from the atmospheric weathering agents. The methodology used in this research to establish a weathering-time relation is likely applicable to areas in other climates as well. 


\section{Chapter 4: Weathering effect on discontinuity properties}

This chapter described the discontinuity development mechanism in thick to very thick bedded sandstone due to weathering and their implication in the engineering works on rock mass. This is based on the article "Weathering effects on discontinuity properties in sandstone in a tropical environment: Case Study at Kota Kinabalu, Sabah Malaysia" (Tating et al., 2015b).

\subsection{Introduction}

Rock mass discontinuities are one of the most important parameters to be assessed for engineering construction in and on rock masses. The discontinuities determine the overall structure (block characteristics) of the rock mass and also control its mechanical behaviour. Weathering processes affect the number of discontinuities in a rock mass as well as their geometric relationships and geotechnical characteristics. In some climatic conditions, rock masses respond very rapidly to weathering actions, thus causing rock masses to deteriorate before the end of the lifetime (say 50 or 100 years) of an engineering structure on or in the rock mass. In some finer-grained rock masses, i.e., fine-grained, sand-sized or finer, significant deterioration due to the rapid formation of mechanical discontinuities may happen within weeks or months after excavation. Understanding the effect of weathering on discontinuities in rock masses improves the options for better predicting the future geomechanical characteristics of the rock mass. Hence, the design of structures in or on rock masses can be adjusted such that the structure will remain stable over the full design lifetime or that suitable remedial measures can be applied to overcome adverse performance.

The main characteristics of a discontinuity are the orientation, spacing, persistence, aperture, surface roughness of discontinuity walls (in some literature also referred to as "irregularities" or "asperities"), and the

presence and type of infill material. Engineering standards for characterization are given in standards such as BS 5930 (1999), ASTM D5878-08 (2008), and ISO 14689-1 (2003), and publications by the International Society for Rock Mechanics (ISRM) (Ulusay and Hudson, 2007). The term "discontinuity" refers to a wide range of mechanical breaks, separation planes or planes of weakness in a rock mass without any specific reference to their natural or artificial origin (Price, 1990). It includes joints, bedding planes, cracks, blasting cracks and fractures, faults and other planar surfaces that have a reduced strength, stiffness, strain softening, or higher permeability relative to the surrounding rock mass (Schultz and Fossen, 2008). In past literature the term "joint" is often used interchangeably with discontinuity; however, in this article the term discontinuity is used 
throughout to define a plane, separation, or break in a rock mass that may or may not have visible displacement.

Natural discontinuities can be the result of periodic sedimentation (i.e., bedding planes), tensile stresses (caused by cooling, desiccation, tectonicrelated tension, or relaxation), shear stresses (caused by uplifting, faulting, folding), and metamorphism processes (such as slaty cleavage, foliation, and schistosity). Some discontinuities, especially shear discontinuities, are normally fully developed and visible in exposures and borehole cores, which allows characterization and measurement of parameter properties to be incorporated in engineering design. However, a vast number of discontinuities, especially those resulting from periodic sedimentation and from metamorphism processes, may be not fully developed and only visible as a flaw in a rock mass. These types of discontinuities will be only become fully developed after the rock mass is affected by weathering and changes in stress environment due to, for example, excavation and stress relief (Hencher and Knipe, 2007). These types of discontinuities are referred to as "integral or incipient discontinuities", which have yet to exhibit a visible break or plane of weakness (Hack, 1998; Hencher, 2013; Price et al., 2009). Integral discontinuities, their possible change into mechanical discontinuities, and the influence of the origin of the discontinuities on the change from integral into mechanical, are often not properly appreciated in site surveys (Hencher and Knipe, 2007).

In Malaysia, most of the studies on rock mass weathering action concern the influence of weathering on the geotechnical properties of the intact rock in engineering applications, and the development of weathering profiles and soil layers over long (geological) time spans (Komoo, 1985; Mohamed et al., 2007; Tating et al., 2013). At present, there is no known study on the effects of weathering or time-related weathering processes on discontinuity properties of thickly bedded sedimentary rock sequences of Kota Kinabalu, Sabah. The purpose of this research is to determine the effect of weathering on the development of discontinuities in thickly to very thickly bedded sandstone in a humid tropical environment. The results of this study are likely to have major implications on design and management of cut slopes, as the behaviour of the rock mass in the future, i.e., during the lifetime of the engineering structure, can be better predicted.

\subsection{Rock mass discontinuities and weathering}

\subsubsection{Integral versus mechanical discontinuities}

Discontinuities can be classified into two basic types based on their appearance and visibility within a rock mass: (1) mechanical and (2) integral 
or incipient discontinuities (Hack, 1998; Hencher, 2013; Slob, 2010). Mechanical discontinuities are flaws or planes of weakness in a rock mass that have low or no tensile strength and lower shear strength compared to the surrounding intact rock material (Ulusay and Hudson, 2007). Most mechanical discontinuities develop from integral discontinuities as a response to changes in stress environment or due to weathering processes (Price et al., 2009). As such, mechanical discontinuities are visible in the rock mass especially if they are open with or without infill. Integral discontinuities have not been subjected to opening and their mechanical properties may differ only slightly from the surrounding intact rock. They are inherent inhomogeneities within intact rock, such as bands of different mineralogical or chemical content, variability in mineral orientation (Slob, 2010), or some partial break or series of breaks forming a weakness pattern (Hencher, 2013). There are circumstances in which mechanical discontinuities become integral discontinuities, for example by the mineralization or cementation of the discontinuity.

\subsubsection{Discontinuity Formation}

Rock mass discontinuities are formed as a consequence of rock mass genesis, the effect of varying stress conditions in and at the earth's surface due either to tectonism or the removal of overburden by erosion or excavation. They are also due to external forces imposed by tectonic processes or overburden weight or from internal forces, such as cooling of igneous rocks (Rawnsley et al., 1990). Therefore, the formation of discontinuities is not a random process (Aydan and Kawamoto, 1990). It is governed by set geological conditions, i.e., sedimentation sequence (McConaughy and Engelder, 2001), stress, fluid pressure, temperature, and fluid-rock interactions during the formation and the geological history of the rock formation up to the recent conditions in which the rock mass is exposed to weathering action at or near the ground surface (Hencher and Knipe, 2007). This is the reason that discontinuities exhibit characteristics such as regular geometric patterns and properties. By origin, Aydan and Kawamoto (1990) classify discontinuities into four main groups, to which Anon (1995) add a fifth:

1. tensional discontinuities,

2. shear discontinuities,

3. discontinuities due to periodic sedimentation,

4. discontinuities due to metamorphism, and

5. discontinuities that develop as a consequence of chemical decomposition and/or physical disintegration due to weathering processes (section Effect of weathering processes on geomechanical properties of rock masses). 


\subsubsection{Discontinuity Sets and Properties}

Discontinuities are normally grouped in sets (or families), where this is a series of discontinuities that have the same genesis, broadly the same geometry, and much the same geotechnical characteristics. Shear zones and faults may also, and often do, occur in a set, but on the scale of engineering works, they usually occur as a single structure. The general methodology for quantitative description of discontinuities is presented by the International Society for Rock Mechanics (Ulusay and Hudson, 2007). Discontinuity properties important in relation to weathering in the context of this paper are geometry (orientation, spacing, and persistence) and shear strength (roughness and infill), where both are discussed in more detail below.

\subsubsection{Discontinuity geometry and pattern}

Orientation, spacing, persistence, and number of discontinuity sets are the main properties determining the geometry of discontinuities in a rock mass (Hack, 1998; Price et al., 2009; Ulusay and Hudson, 2007; Wyllie and Mah, 2004). These determine the size and shape of discontinuity bounded rock blocks and the possibilities for relative movement along discontinuities. The orientation of discontinuities relative to the direction of imposed stresses is important in engineering. Its importance increases with the presence of other conditions conducive to failure such as low shear strength and suitable discontinuity sets. Discontinuity spacing is defined as the perpendicular distance between two adjacent discontinuities that belong to the same set and it is usually reported as the mean or characteristic spacing of a discontinuity set. Discontinuity persistence is the extent of a discontinuity along the strike and dip directions of a discontinuity. Three types of discontinuity persistence are distinguished: (1) Persistent discontinuities that are continuous across the exposed rock mass or geotechnical unit; (2) Abutting discontinuities that terminate against other discontinuities; and (3) Non-persistent discontinuities that terminate within intact rock. Movement along a non-persistent discontinuity can only take place after the intact rock (i.e., a "rock bridge") is broken. Studies regarding the determination of discontinuity geometry by different methods are presented by Hack (1998), Lemy and Hadjigeorgiou (2003), Priest (2004).

\subsubsection{Discontinuity shear strength}

Discontinuity roughness (or unevenness or size and number of "asperities") refers to the degree of irregularity of the discontinuity surface. It greatly influences the shear strength of discontinuities. Roughness can be classified into various classes depending on scale. A commonly used classification is for large-scale (on surfaces of the order of metre size), small-scale (on surfaces of the order of centimetre size), and material roughness (invisible) (Hack et al., 2003). Roughness affects the shear strength of the discontinuity and the 
large-scale roughness (also named waviness or shape of the discontinuity) may affect the initial direction of shear displacement (Ulusay and Hudson 2007). The role of roughness in controlling the shear strength of discontinuities has been the subject of extensive research (such as Bandis, 1990; Barton, 1973; Hack and Price, 1995; Kulatilake et al., 1999; Patton, 1966; Ulusay and Hudson, 2007; Yang et al., 2001), which has resulted in the development of various shear strength criteria for rock discontinuity roughness, such as SSPC system (Hack et al., 2003) that has been used in this research.

Discontinuity infill refers to the material that separates the adjacent rock walls of discontinuities (Ulusay and Hudson, 2007). Infill material influences the shear resistance of a discontinuity by: (1) reducing the micro-roughness (reducing the effect of surface textural interlocking), (2) changing the basic frictional properties of the shear surface based on the relative values of particle friction of the infill and the discontinuity wall material, and (3) reducing the effective roughness by changing the morphology of the shear surface (Papaliangas et al., 1993). The shear strength of discontinuities with infill is completely or largely determined by the thickness of the infill and by the material properties of both infill and material of the discontinuity wall as well as its roughness (i.e., the height and shape of the asperities).

Several studies have been done to determine the influence of infill on discontinuity shear strength, for example, Amin et al. (2008); Indraratna et al. (2010); Papaliangas et al. (1993); Pereira (1990); Phienwej et al. (1990); Zare et al. (2008). Generally, these studies have shown that shear strength of filled discontinuities is dependent mainly on the thickness of infill in relation to the amplitude of the roughness, the height and shape of asperities, and on the geotechnical properties of the infill material; i.e., the thicker the infill, the lower the shear strength, and presence of materials with low shear strength such as clay or talc, that result in a low shear strength. On the other hand, an infill comprising quartz grains would probably possess relatively high shear strength.

\subsubsection{Effect of weathering processes on geomechanical properties of rock masses}

Generally, weathering of rock masses causes reduction of intact rock strength, the breakdown into smaller fragments of larger blocks of intact material, and the forming of infill material in discontinuities. The breakdown into smaller fragments of larger blocks of intact material is usually due to integral discontinuities becoming mechanical (Ehlen, 2002; Fell et al., 2012; Hack and Price, 1997; Hencher, 2013). Fresh intact rock upon exposure to weathering agencies will undergo two main processes of alteration: discoloration and decomposition by chemical weathering and disintegration 
by physical weathering. Often, biological processes are considered an additional agency, but this is disputable because the influences are also chemical and/or physical. Decomposition processes may produce weathered material with a composition entirely different from the original rock and also it may also result in pronounced colour changes to rock and rock surface. On the other hand, disintegration may result in the breakdown of rock material into progressively smaller fragments without any noticeable change in mineral constituents (Selby, 1993). The effects of weathering action on the geomechanical properties of various rock types have been studied by many researchers, such as Ehlen (2002); Gupta and Rao (2001); Hack and Huisman (2002); Hack and Price (1997); Jeong et al. (2005); Undul and Tugrul (2011).

\subsection{Methodology}

This study is primarily based on field data, the quality of which is dependent on how systematically the conditions were observed, collected, and arranged. Discontinuity data has been collected and organized following the systematic approach of the slope stability probability classification (SSPC) methodology (Hack et al., 2003) that encompasses field assessment of slope stability and quantified rock mass properties (see Section $\mathbf{1 . 6}$ for methodology details).

\subsubsection{Data Analysis}

All discontinuities (joints and bedding planes) were plotted as poles on an equal-area stereogram using Dips (2012) software, contoured on density, and analysed using Fisher distributions. The weathering grade was then assigned to each discontinuity set and each discontinuity in a set to facilitate analyses of the relationships between weathering and variation of discontinuity spacing.

\subsection{Results}

\subsubsection{Discontinuity sets}

The discontinuity sets are split into subsets (Table 4.1) depending on the direction of dip of the different limbs of folds. In Figure $\mathbf{4 . 1}$, the concentration of poles for bedding planes and discontinuity Set 2 are close together and it could be argued that these form one concentration cloud; however, according to Tating (2003) it is more appropriate to regard these as two sets and, therefore, this division is maintained.

Three main discontinuity sets have been identified in the SST unit (Figure 4.2 and Figure 4.3), as listed in Table 4.1, where Set 1 is the bedding planes of the SST unit, Set 2 is conjugate joint sets, which are oriented more 
or less parallel (NE-SW) to the dip directions of the bedding planes (Set 1 ), but with a less steep dip, (bedding planes $74^{\circ}-80^{\circ}$, discontinuities: $20^{\circ}-$ $\left.37^{\circ}\right)$. Set 3 consists of steeply dipping joints $75^{\circ}-77^{\circ}$, either NE or SW that strike more or less perpendicular (NE-SE) to the bedding planes.

Table 4.1 Discontinuity sets in SST unit.

\begin{tabular}{|c|c|c|c|}
\hline \multicolumn{2}{|c|}{ Discontinuity } & \multirow{2}{*}{ Dip/Dip-direction $\left(^{\circ}\right)$} & \multirow{2}{*}{ Description } \\
\hline Set & Subset & & \\
\hline \multirow{2}{*}{1} & $1-\mathrm{I}$ & $74 / 311$ & \multirow{2}{*}{$\begin{array}{l}\text { Bedding planes. Steeply dipping either } \\
\text { towards the SE or NW }\end{array}$} \\
\hline & $1-\mathrm{II}$ & $80 / 133$ & \\
\hline \multirow[t]{2}{*}{2} & 2-I & $20 / 310$ & \multirow{2}{*}{$\begin{array}{l}\text { Conjugate joint sets. Joint planes; strike } \\
\text { more or less parallel to bedding planes. } \\
\text { Gently dipping towards the SE or NW }\end{array}$} \\
\hline & 2-II & $37 / 134$ & \\
\hline \multirow{2}{*}{3} & 3-I & $77 / 048$ & \multirow{2}{*}{$\begin{array}{l}\text { Joint planes; strike more or less } \\
\text { perpendicular to bedding planes. Steeply } \\
\text { dipping towards the SW or NE }\end{array}$} \\
\hline & 3-II & $75 / 227$ & \\
\hline
\end{tabular}

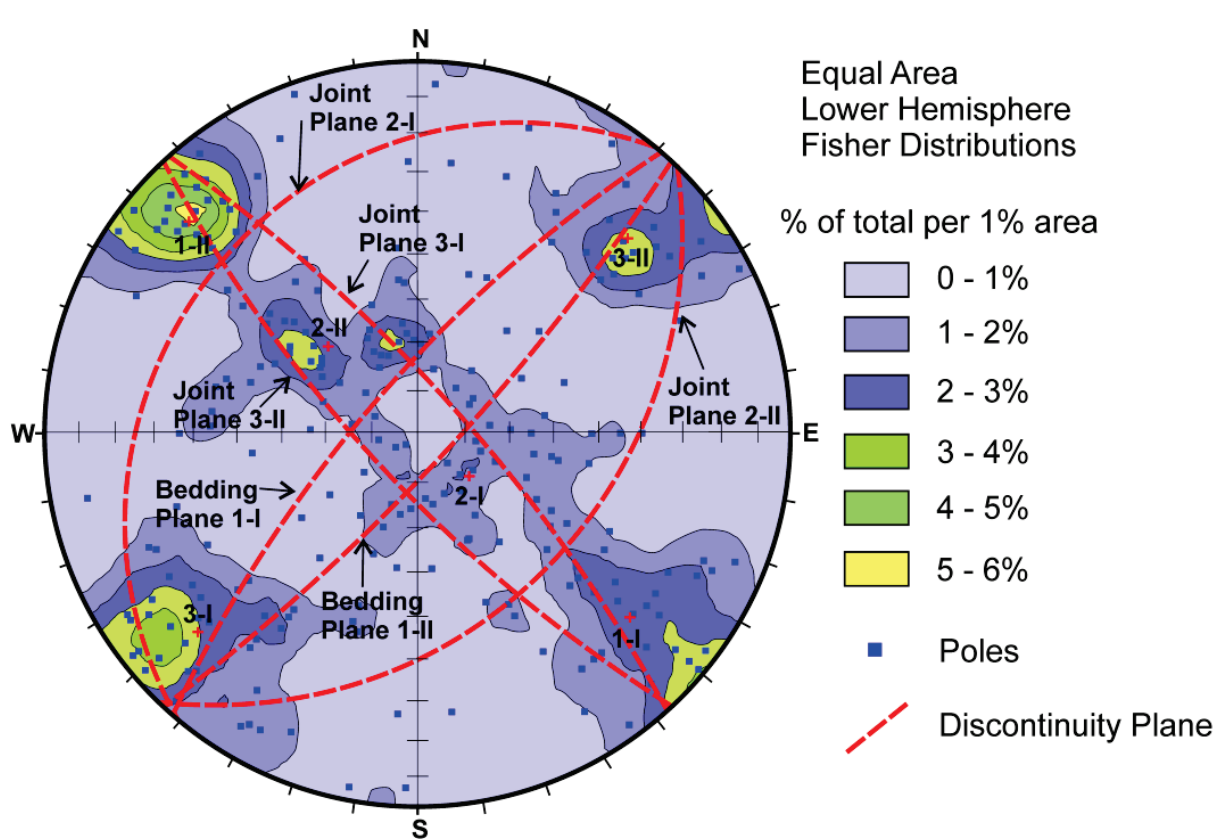

Figure 4.1 Equal area, lower hemisphere plot of discontinuity poles with Fischer distributions and mean discontinuity planes per set, all plotted on lower hemisphere in Dips (2012).

\subsubsection{Discontinuity spacing}

The influence of weathering action on the formation of new discontinuities is determined by comparing the mean and median spacing of each discontinuity 
set per weathering grade. The results of the analyses are shown in Table $\mathbf{4 . 2}$ and Figure 4.3 from which it is clear that the mean and median spacing for all discontinuity sets decrease with increasing weathering grade. Figure 4.4 shows the formation of new mechanical discontinuities in the SST unit induced by weathering. The decrease of discontinuity spacing is due to the formation of new mechanical discontinuities that become visible (Figure 4.4c).

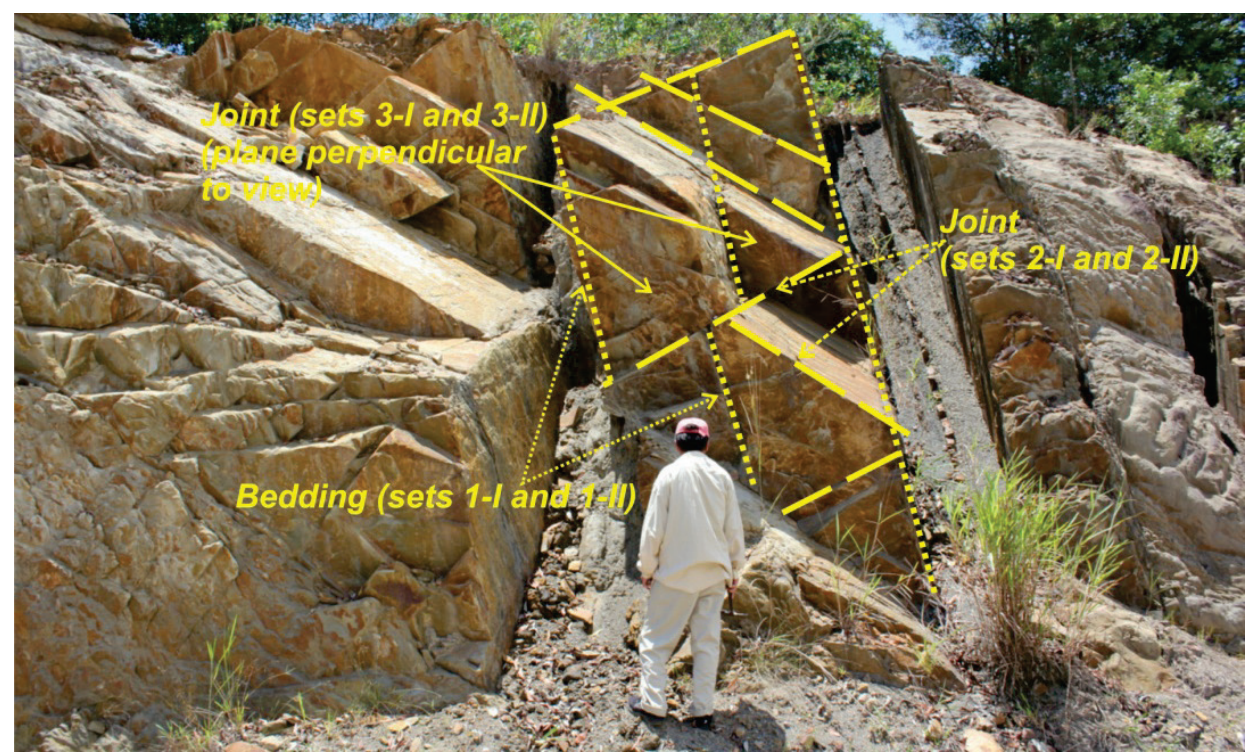

Figure 4.2 Discontinuity sets in the thickly to very thickly bedded SST unit in a cut slope.

\subsubsection{Discontinuity sequential development}

The sequential development of discontinuities with advancing weathering grade is shown in Figure 4.5, where Figure 4.5a-d show this in a stereogram, whereas Figure $\mathbf{4 . 5 e - h}$ show the same information as a schematic model. The analyses demonstrate that the discontinuity formation pattern is directly related to the weathering grade. In the fresh state, the noticeable discontinuities are the bedding planes (discontinuity Set 1) as clearly marked by the concentrations poles in the northeastern and southwestern parts of the stereogram (Figure 4.5a), and the very few discontinuities in different orientations probably result from the residual in situ stresses. In slightly weathered rock (Figure 4.5b), the intensity of planes of discontinuity Set 1 (bedding) increases while the concentrations of discontinuity Set 2 (conjugate joint sets) also start to become clearly marked. Few new planes belonging to a new discontinuity set oriented perpendicular to the bedding planes (discontinuity Set 3; also conjugate joint sets) are formed and become vaguely noticeable in Figure 4.5b. In 
moderately weathered rock, the discontinuity intensity for all discontinuity sets significantly increases (Figure 4.5c), and these increase further in highly weathered masses (Figure 4.5d). The frequency distribution of the discontinuity spacing per weathering grade for all discontinuity sets together is shown in Figure 4.6, which demonstrates that, especially for discontinuity spacing $<0.5 \mathrm{~m}$, the frequencies increase with higher weathering grades.

Table 4.2 Comparison of mean and median discontinuity spacing per set and weathering grade. "All" means that the values are for all sets together irrespective of to which set the plane belongs.

\begin{tabular}{|c|c|c|c|c|c|}
\hline \multirow{2}{*}{$\begin{array}{l}\text { Discontinuity } \\
\text { set }\end{array}$} & \multirow{2}{*}{$\begin{array}{l}\text { Weathering } \\
\text { grade }^{a}\end{array}$} & \multirow{2}{*}{$\begin{array}{l}\text { Number } \\
\text { of planes }\end{array}$} & \multicolumn{3}{|c|}{ Spacing (m) } \\
\hline & & & Mean & $\begin{array}{l}\text { Standard } \\
\text { deviation }\end{array}$ & Median \\
\hline \multirow[t]{5}{*}{1 (Bedding) } & Fresh & 2 & 1.20 & 1.13 & 1.2 \\
\hline & Slightly & 2 & 0.70 & 0.42 & 0.7 \\
\hline & Moderately & 16 & 0.51 & 0.39 & 0.4 \\
\hline & Highly & 22 & 0.28 & 0.33 & 0.16 \\
\hline & Completely & 2 & 0.025 & 0.01 & 0.025 \\
\hline \multirow[t]{5}{*}{2 (Joint) } & Fresh & 4 & 1.03 & 0.55 & 1.05 \\
\hline & Slightly & 13 & 0.50 & 0.29 & 0.45 \\
\hline & Moderately & 27 & 0.36 & 0.30 & 0.3 \\
\hline & Highly & 31 & 0.21 & 0.13 & 0.15 \\
\hline & Completely & 2 & 0.035 & 0.21 & 0.035 \\
\hline \multirow[t]{5}{*}{3 (Joint) } & Fresh & 4 & 0.71 & 0.33 & 0.73 \\
\hline & Slightly & 3 & 0.20 & 0.10 & 0.2 \\
\hline & Moderately & 16 & 0.15 & 0.11 & 0.11 \\
\hline & Highly & 17 & 0.10 & 0.07 & 0.07 \\
\hline & Completely & 3 & 0.02 & 0.01 & 0.02 \\
\hline \multirow[t]{5}{*}{ All } & Fresh & 10 & 0.94 & 0.57 & 0.8 \\
\hline & Slightly & 18 & 0.48 & 0.30 & 0.4 \\
\hline & Moderately & 59 & 0.34 & 0.32 & 0.3 \\
\hline & Highly & 71 & 0.26 & 0.21 & 0.15 \\
\hline & Completely & 7 & 0.03 & 0.12 & 0.02 \\
\hline
\end{tabular}

\subsubsection{Discontinuity persistence}

The persistence of the bedding planes in the SST unit is controlled by the thicknesses of the original strata. The bedding planes that form a mechanical discontinuity are normally persistent. However, the transformation from integral into mechanical discontinuities may not be uniform in time over the whole extent of the discontinuity surfaces. Therefore, a bedding plane may have become in places a mechanical discontinuity, whereas it still is an integral discontinuity at other locations along the same plane. Over the whole unit, this effect is less important as the transformation of integral into mechanical discontinuities is often en echelon, hence the number of bedding planes being mechanical discontinuities at any one place in the same unit with the same weathering grade is about the same. Discontinuity Sets 2 and 3 normally abut against Set 1 (bedding planes) because the bedding planes become mechanical before a joint has been transformed from integral into mechanical, and Set 1 serves to relieve tensile stresses (Figure 4.2). 


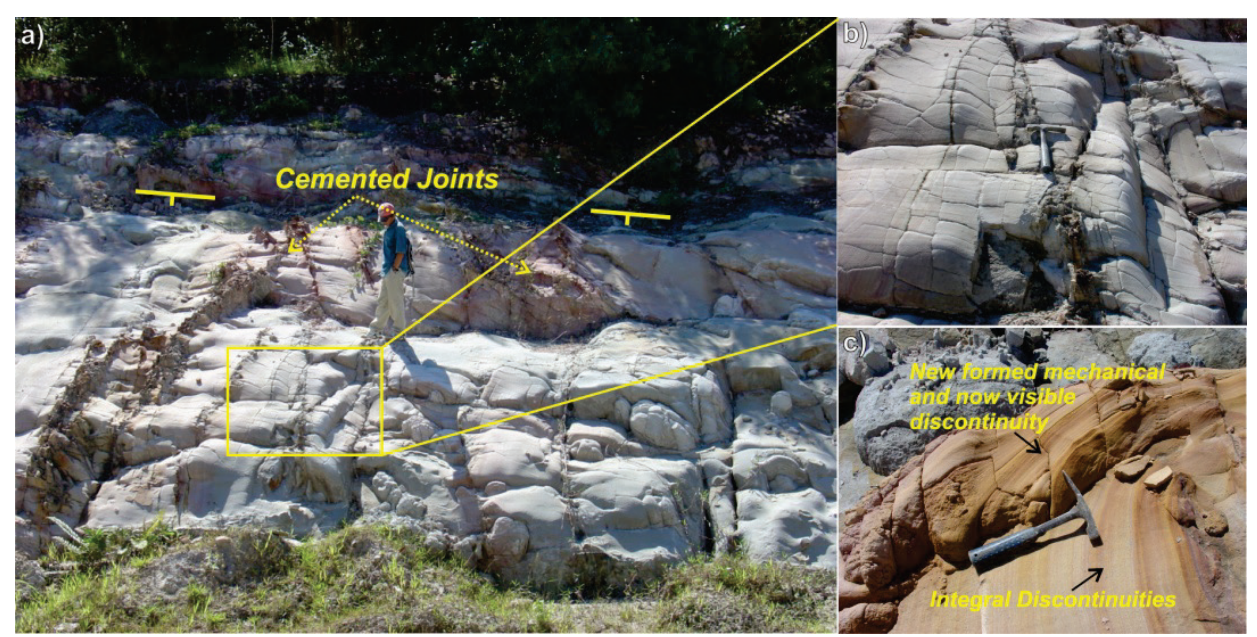

Figure 4.3 a) Joint system in SST unit, some of the joints are cemented. SST unit bedding orientation is shown by the strike/dip symbol; b) Detailed joint system induced by weathering; $c$ ) Newly visible bedding plane in the SST unit (length of hammer $\approx 0.3$ $\mathrm{m})$.

\subsubsection{Discontinuity roughness}

Most bedding or joint discontinuities have a large-scale roughness of "straight" independent of weathering grade. The small-scale roughness is mostly "smooth undulating" in grade fresh masses and becomes "rough planar" in more weathered masses. This is contrary to what is expected as mostly, small-scale roughness becomes smoother due to the deterioration of asperities (Geertsema, 2003). However, roughness may be increasing with increasing degree of weathering in tropical areas, if chemical weathering is dominant and precipitation of iron hydroxide and other iron compounds along the discontinuities occurs. In the study area, such coatings are abundant and are a notable feature of the discontinuities in the SST unit. Precipitation of these coatings on the surfaces of the discontinuities results in very rough surfaces and sometimes in completely cemented discontinuities. These ironcoated or cemented discontinuities are found more in moderately to highly weathered sandstone than in sandstone with a lower degree of weathering (Figure 4.7). The coating or cement is very hard and between 0.1 and 0.5 $\mathrm{mm}$ in thickness. Discontinuities that are neither coated nor cemented with iron compounds are mostly filled with fine-grained clayey material that probably results from weathering. These discontinuities have a smoother small-scale roughness with a higher degree of weathering. 

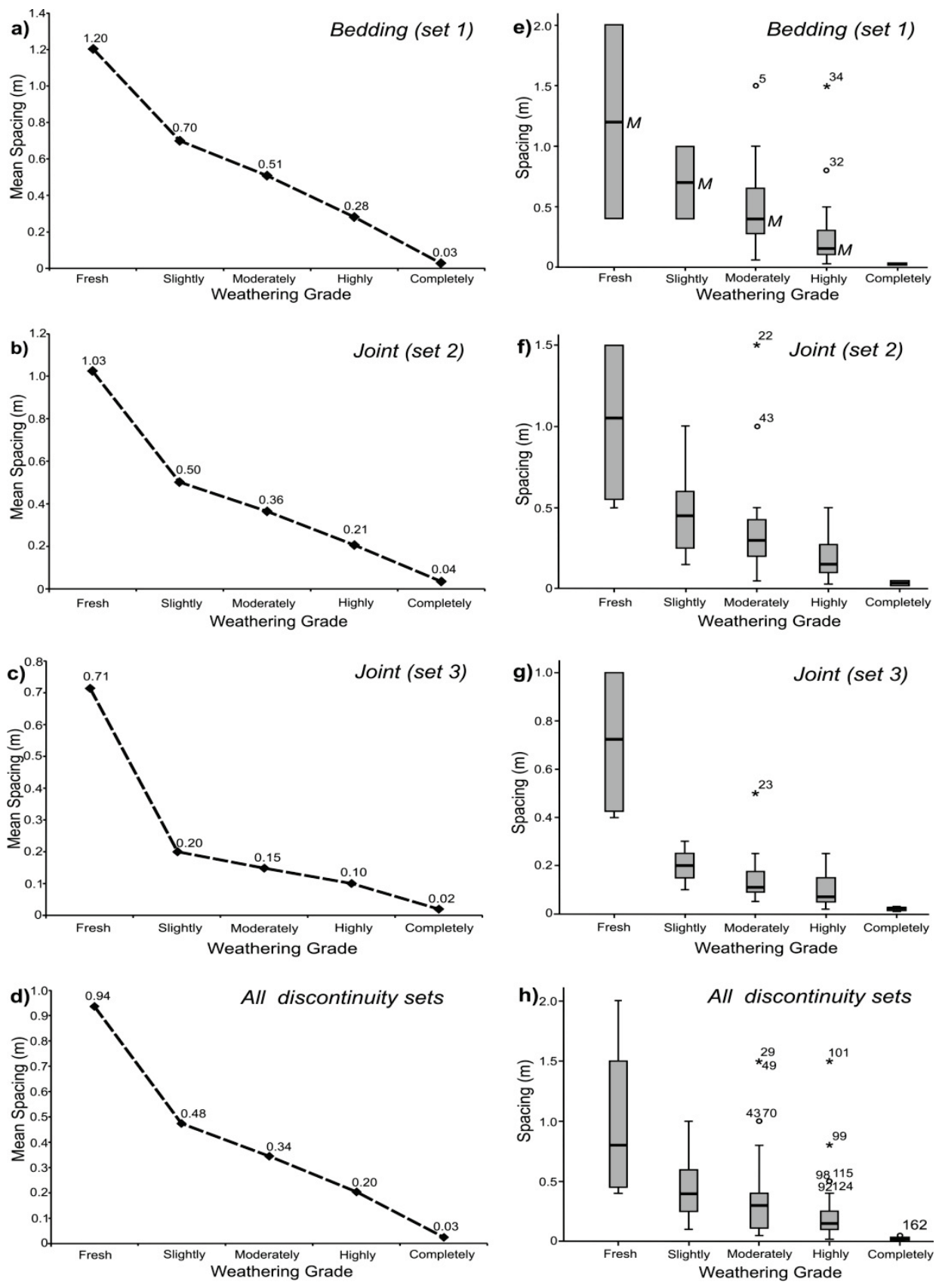

Figure 4.4 Mean $(a-d)$ and boxplot $(e-h)$ of discontinuity spacing decreases with higher weathering grades. $M$ indicates median value in the boxplot. 

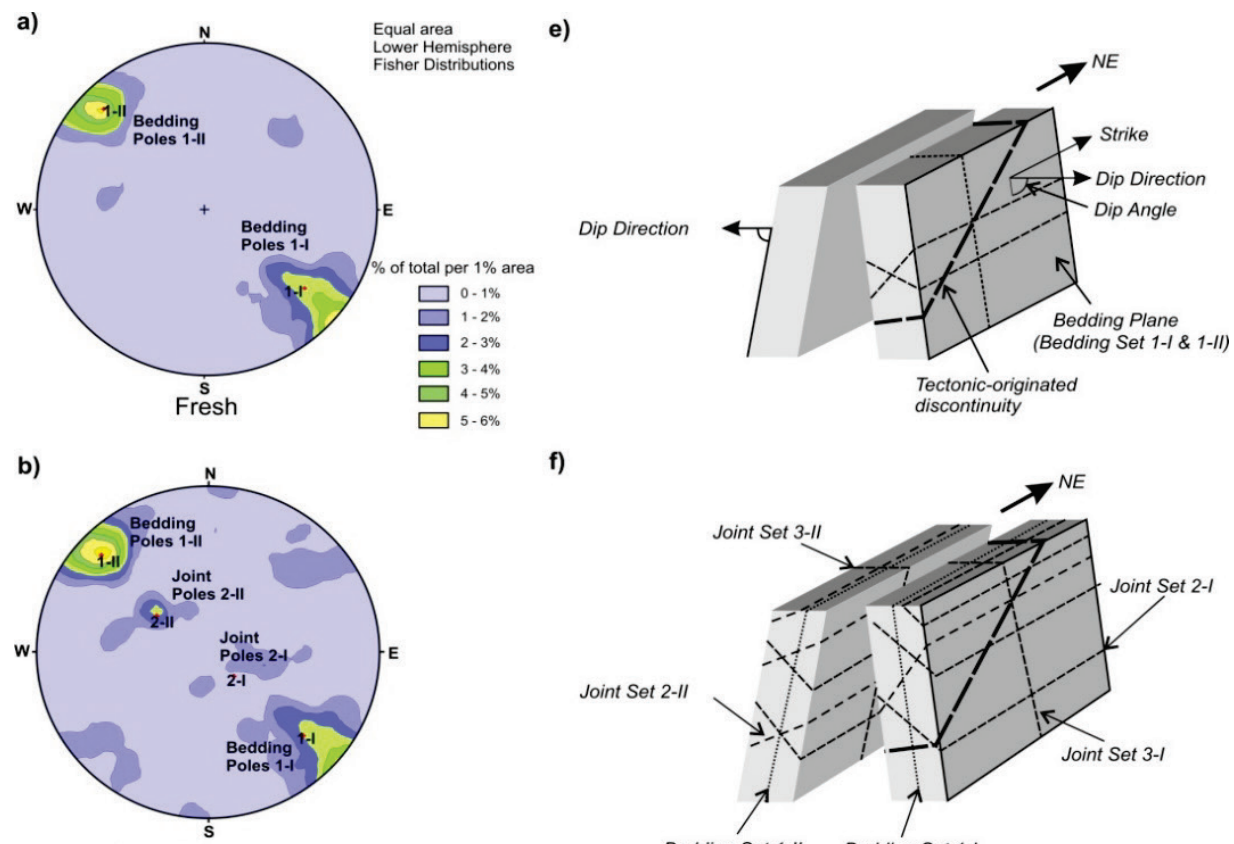

f)
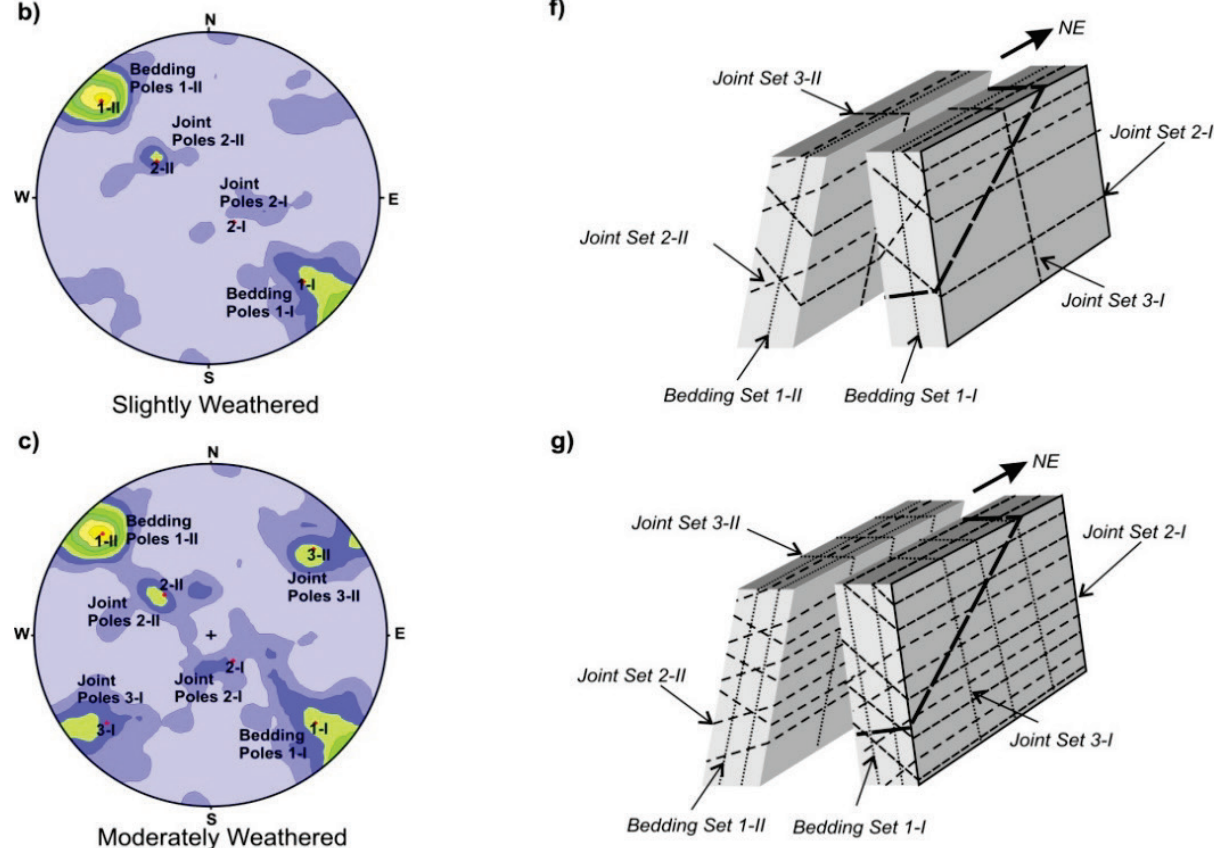

d)

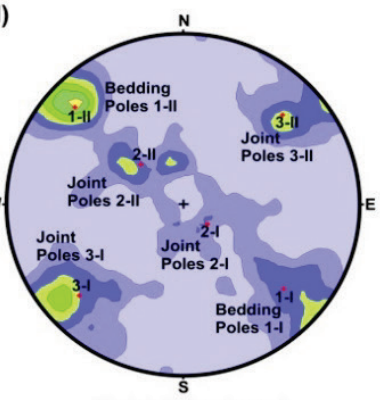

h)

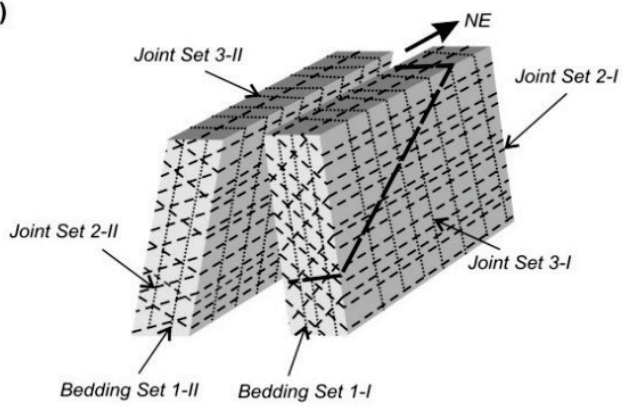

Figure 4.5 a - d Plots of discontinuity poles per weathering grade; e - $\mathrm{h}$ schematic model showing the development of discontinuity sets in the steeply dipping SST unit. 


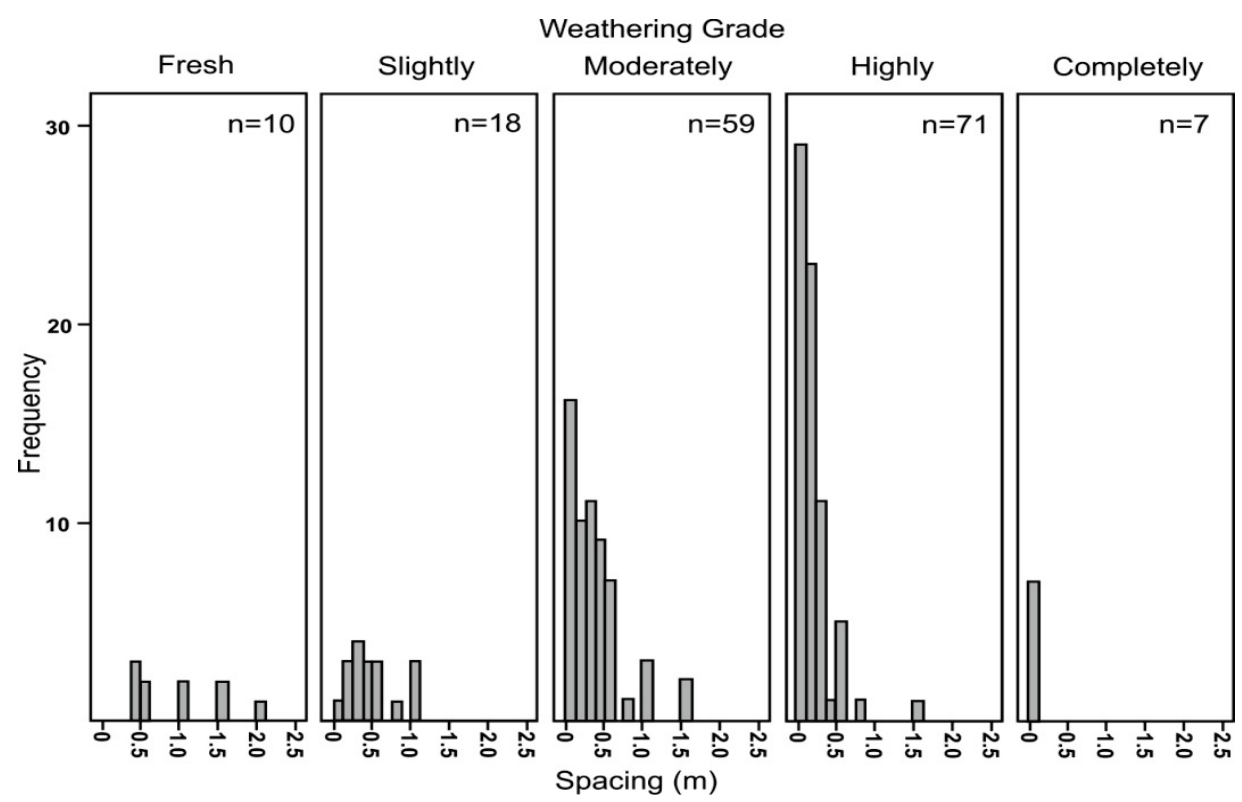

Figure 4.6 Frequency of discontinuity spacing distribution per weathering grade for all discontinuity sets together.

\subsection{Discussion}

There are two important effects of weathering processes related to discontinuities in the thickly to very thickly bedded SST unit: (1) decreasing discontinuity spacing with increasing degree of weathering and (2) sequential development of three dominant discontinuity sets. The spacing decreases due to the change from integral into mechanical discontinuities. This is in agreement with the literature (Ehlen, 2002; Hack and Price, 1997).

In the thickly to very thickly bedded sandstone unit, integral discontinuities may be in the form of laminations (either parallel or cross) or other sedimentary internal bedding features such as the "Bouma Sequence" (Bouma, 1962), trace fossils, nodules, concretions, and shale laminae. These features have been observed in the fresh intact rock of the SST unit and are likely initiation points for discontinuity formation in bedded clastic rocks (McConaughy and Engelder, 2001; Pollard and Aydin, 1988). In addition, integral discontinuities may also be formed due to the presence of invisible internal structures (Hencher and Knipe, 2007). For example, weathering processes may cause alteration or dissolution of specific minerals that form the integral discontinuities, which subsequently develop into new mechanical discontinuities. Moreover, new discontinuities may also develop due to different elastic response of adjacent minerals grain to changes in geological environment, such as loading and unloading during wetting and drying of the rock mass (Hencher and Knipe, 2007; Huisman et al., 2004). Such processes 
also cause volume changes that may generate internal stresses in the rock mass. All these processes are liable to result in reducing the spacing of discontinuities as the degree of weathering increases as Figure 4.3 shows.
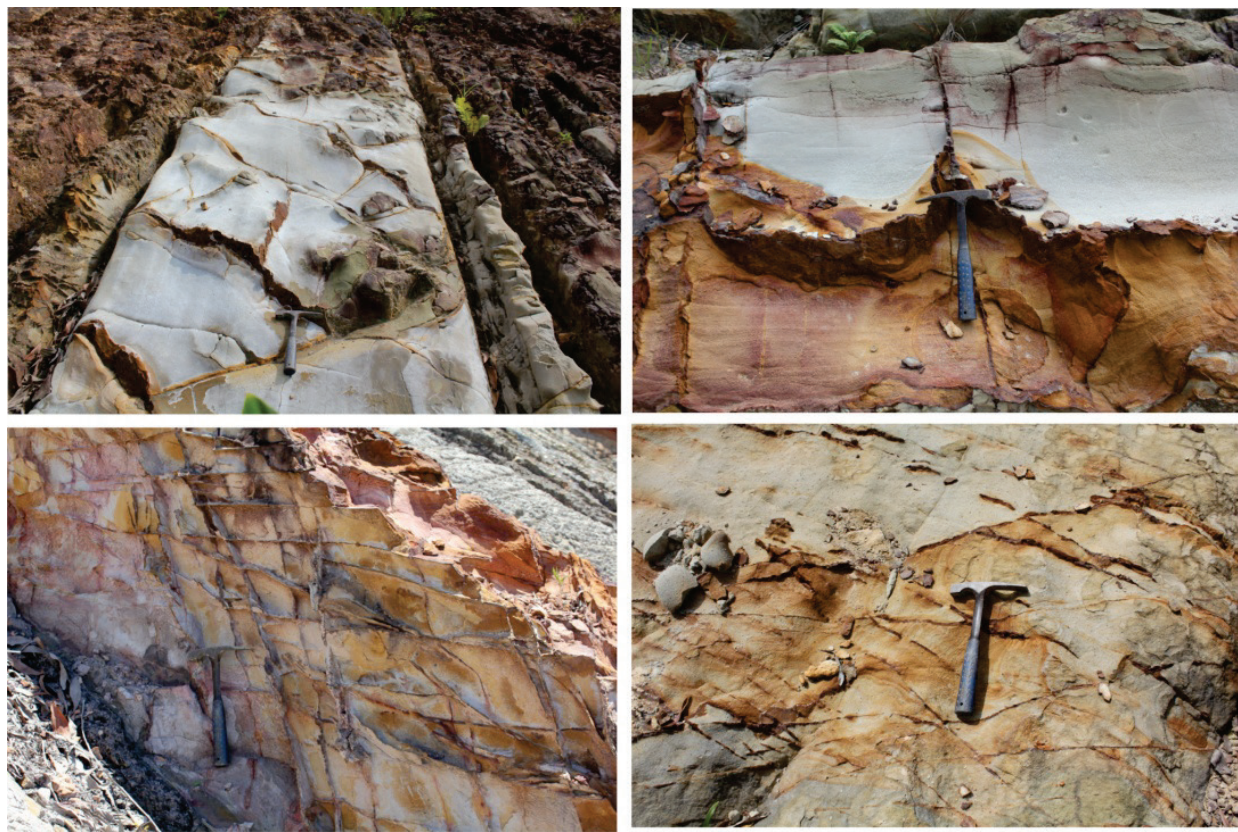

Figure 4.7 Iron cemented joints in SST unit (length of hammer $\approx 0.3 \mathrm{~m}$ ).

Changes in stress distribution in magnitude and orientation may also contribute to the formation of new discontinuities. When a rock mass is excavated, the stress due to the overburden reduces or becomes nil, and residual; in situ horizontal stresses are reduced. This stress reduction and change in orientation of the stress axes may result in the formation of new discontinuity sets (Figure 4.8).

In the study area, the in situ major principal stress $(\sigma 1)$ is probably horizontal and oriented at about NW-SE (Tongkul, 1990). The new (i.e., due to weathering processes) discontinuities do not develop with equal intensity in each set. When fresh, the discontinuities belonging to the bedding plane (Set 1) are dominant with some conjugate discontinuities due to the regional in situ stress. As the rock mass becomes more weathered, new discontinuities are developed particularly in Sets 2 and 3 with relatively less additional discontinuities in Set 1 . Relationships between historical stress environments and the development of patterns and intensities of discontinuities have also been recognized by Fernandes-da-Silva et al. (2010) and used for drainage pattern and landscape recognition. 


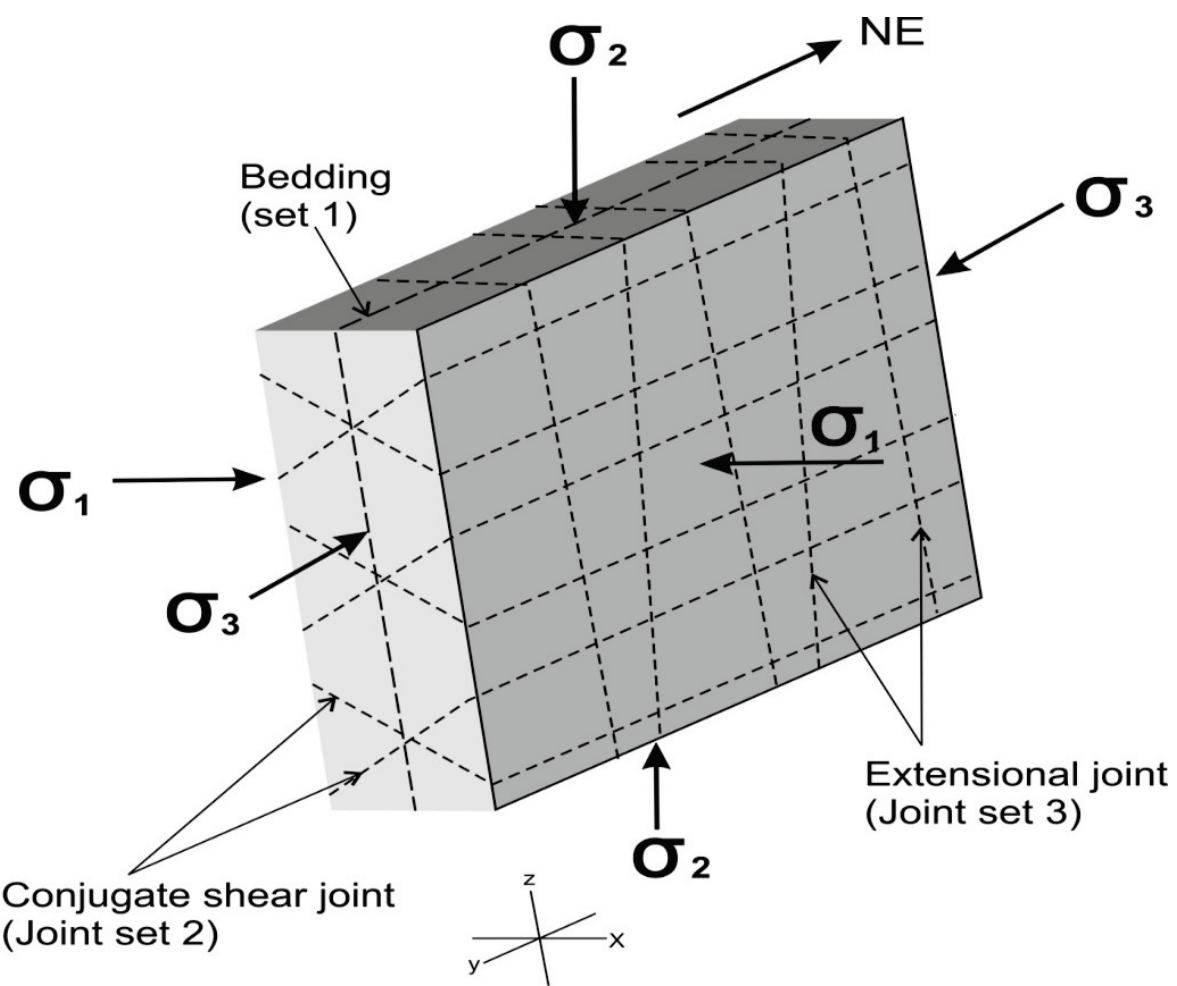

Figure 4.8 Development of joint sets under a three-dimensional stress regime. The conjugate joint sets (set 2 ) and extensional joints (set 3 ) may be developed from the main principal stress $(\sigma 1)$.

Discontinuity roughness and infill also have a significant influence on the mechanical characteristics of a rock mass. In the current study, it was found that weathering did not have much effect on the large-scale roughness; however, it did have an influence on the small-scale roughness. Weathering of the discontinuity surfaces reduces the strength and number of asperities resulting in smoother surfaces that possess lower shear strength. Secondly, the material weathered from the discontinuity walls will become infill material. In many locations, the discontinuities in the moderately and highly weathered SST units are coated or cemented with iron oxides. Infill material is normally a reason for lower shear strength; however, strongly compacted or cemented infill may cause an increase in shear strength. The possibility of infill material having been washed into discontinuities near to the top or surface of an excavation cannot be fully excluded; however, this is unlikely to be the source of the infill material in the majority of the discontinuities assessed for this study. The openness of the discontinuities is rather small and evidence of material washing over the excavation surface was not observed at the locations where the data were collected. The influence of vegetation is likely to be small or absent. Root growth into discontinuities 
from vegetation at the top of excavations prior to excavation is limited to the upper few metres of the rock mass and almost never at locations where the data for this research were collected. Excavation surfaces were mostly free of newly grown vegetation and if present, it consisted only of small plants up to maximum 10-30 cm high and occasionally fast growing trees such as acacia (see Figure 4.2, Figure 4.3, and Figure 4.7).

The effects of weathering action on discontinuities as described are determined in a particular type of sandstone and for weathering in a tropical humid climate. The decrease in discontinuity spacing is also observed in other types of rock masses and climates. However, the sequential discontinuity formation and the orientation of newly developed discontinuities observed in this study may not be applicable to other types of rocks or in places where the stress history is different. Integral discontinuities present within a rock mass are the result of the internal structure of the rock mass and the stress conditions during its geological history. Furthermore, rock weathering is controlled by many factors (Bland and Rolls, 1998) many of which are site specific, and hence, even in the same climate, the effect on a rock mass may be different. Although this study is location-dependent it shows that if the geological history (e.g., sedimentation process and tectonic history) of a rock mass is known, in combination with a detailed study of the rock mass, it is likely possible to forecast the reduction of spacing with ongoing weathering of already existing mechanical discontinuity sets (as is also shown by Hack et al., 2003; Huisman et al., 2006) and the likely initiation of new sets. Moreover, it is unusual for one weathering grade to be present in an exposure, so in most cases it will be possible to find exposures, perhaps outside the actual construction site, where the same rock mass in the same geological environment is exposed at a higher weathering grade. Such exposures can be used also to study the possible development of new mechanical discontinuity sets.

\subsection{Conclusions}

Mechanical discontinuity sets are formed with increasing weathering grade in thickly to very thickly bedded sandstones from integral discontinuities that are invisible in fresh sandstone. Furthermore, discontinuity spacing decreases with higher grades of weathering as expected, and it is probable that new mechanical discontinuities are formed from integral (or incipient) discontinuities as weathering action progresses and in response to changes in the stress environment brought about by excavation and engineering work. In the case study, three main sets of discontinuities are developed: the bedding discontinuities, discontinuities that have about the same orientation as the bedding planes and a set of conjugate discontinuities with a strike parallel to the bedding but with a lower dip angle, and discontinuities with a 
strike perpendicular to the bedding planes. The sequential development of these discontinuity sets is related to the weathering grade. In a fresh rock mass, the discontinuities are often non-persistent and terminate within the SST strata. In higher weathering grade rock masses, the discontinuities become more persistent and often abut against bedding planes. Discontinuity persistence parallel to the bedding is longer in higher weathering grades and discontinuities intersect discontinuities of other sets. It must be noted that the effect of weathering is observed in thickly bedded sandstone units in a tropical humid environment. As the effects of weathering are dependent on rock mass type, geological history, and local climate, the same effects may not always occur in other types of rock masses or other areas; however, it is likely that in similar rock masses similar effects will occur.

There are important implications for engineering of newly developed mechanical discontinuity sets that were not visible at the design stage of construction because adjustments to the designs are needed that anticipate the formation of a less intact rock mass over the engineering lifetime. Generally speaking, such considerations are usually ignored; however, this research shows that the effects of weathering processes on rock mass properties can be predicted in terms of an increase in the number of discontinuity sets as well as an increase of the number of discontinuities in each set. It also demonstrates that it is possible to predict the future pattern and spacing of discontinuities from studies of the integral or incipient discontinuities present, although this possibility needs to be further tested in different geological and climatic situations. 


\section{Chapter 5: Influence of weathering-induced iron precipitation on sandstone properties}

This chapter described the influence of weathering in the development of weathering zones and the iron precipitation along the discontinuities in thick bedded to very thick-bedded sandstone. The influence of iron precipitation on the condition of discontinuities is also discussed. This chapter is based on the articles "Influence of weathering-induced iron precipitation on properties of sandstone in a tropical environment" (Tating et al., 2015b; submitted for publication).

\subsection{Introduction}

Bedding planes, cleavage schistosity, joints, and fracture planes, or more general, "discontinuities" have a major influence on the hydro-mechanical properties of rock masses. For example, roughness of the discontinuity walls and infill material in the discontinuity determine the shear strength of a discontinuity, but also influence the permeability and deformability of the rock mass. The properties determining the hydro-mechanical behaviour of a discontinuity are normally denoted the "condition of discontinuity" which is an aggregate term for all properties related to the hydro-mechanical behaviour of a discontinuity. The importance of the condition of discontinuities in rock masses is extensively discussed in the literature (e.g. Barton, 1973; Barton \& Stephansson, 1990; BS 5930:1999; Goodman, 1989; Hack, 1998; Hencher \& Knipe, 2007; Hoek \& Bray, 1981; ISO 14689-1:2003 ; Laubscher, 1990; Lee \& Hencher, 2009; Palmstrøm, 1975; Patton, 1966; Price et al., 2009; Tating et al., 2014; Ulusay \& Hudson, 2007; Vincente Silvestre et al., 2002).

The condition of a discontinuity is affected by weathering processes especially chemical weathering. The weathering starts from the discontinuity walls due to water with dissolved chemicals percolating through discontinuities. The weathering normally weakens the intact rock material forming the walls of the discontinuity and the asperities on the discontinuity walls. Moreover, the minerals of the discontinuity walls and asperities may be transformed into other minerals, which then may become, for example, clay infill in the discontinuity. Weathering may also cause the change of integral or incipient discontinuities into mechanical discontinuities (Hack, 1998; Hencher, 2013; Price et al., 2009; Slob 2010; Tating et al., 2014). Chemical weathering may have also a reversed effect on the condition of a discontinuity if particular minerals are precipitated by percolating groundwater. Especially in a tropical environment with an undulating morphology, iron may be dissolved or precipitated in specific zones or layers with fairly clearly defined boundaries. These zones or layers are not necessarily equivalent to the weathering grades 
as defined by standards for rock mass weathering (e.g. BS 5930:1999; ISO 14689-1:2003).

The result of this study emphasises that forecasting the susceptibility to weathering of rock masses should take into account climate and morphology of the environment. Just assuming that weathering reduces, for example, the shear strength of discontinuities and intact rock strength, as is often done, may be incorrect.

\subsection{Condition of discontinuity}

The condition of a discontinuity is governed by five main parameters: (1) discontinuity wall roughness, (2) matching of discontinuity walls, (3) strength of the discontinuity walls and asperities on the walls, including alteration by, for example, weathering, (4) infill material in the discontinuity, and (5) discontinuity persistence, which is the length and continuity of a discontinuity. The discontinuity wall roughness can be further divided into large-scale roughness (often denoted "waviness"), small-scale roughness ("unevenness"), and the roughness of the discontinuity wall on microscopic scale ("material roughness"). The shear strength of a discontinuity with infill is governed by the thickness of the infill, the material properties of both infill and the discontinuity walls, the roughness of the discontinuity and shape and height of asperities on the discontinuity walls, and the stress condition on the discontinuity. Several studies have been done to determine the influence of infill and roughness on discontinuity shear strength (Amin et al., 2008; Archambault et al., 1990; Bandis, 1990; Barton, 2013; Barton, 1973; Barton \& Choubey, 1977; Ghazvinian et al., 2010; Hack, 1998; Hack et al., 2003; Hencher \& Richards, 1989; Indraratna et al., 2010; Indraratna et al., 2011; Karami \& Stead, 2008; Kulatilake et al., 1999; Kulatilake et al., 1995; Papaliangas et al., 1993; Papaliangas et al., 1990; Patton, 1966; Pereira, 1990; Phien-wej et al., 1990; Seidel \& Haberfield, 1995; Ulusay \& Hudson, 2007; Yang et al., 2001; Zare et al., 2008). Various strength criteria for discontinuities were determined of which Barton's inter-block shear strength (Barton et al., 1974) and the sliding angle following the sliding criterion (Hack \& Price, 1995) are used in this study to show the influence of dissolution and precipitation of iron on shear strength.

\subsubsection{Barton's discontinuity inter-block shear strength}

The Q - system of rock mass classification (Barton, 2013; Barton et al., 1974 ) is an assessment of rock quality based on six parameters; Rock Quality Designations $(R Q D)$, number of discontinuity sets $\left(J_{n}\right)$, discontinuity roughness $\left(J_{r}\right)$, degree of alteration of discontinuity walls and type of fill in discontinuities $\left(J_{a}\right)$, stress of water in discontinuities $\left(J_{w}\right)$, and stress condition $(S R F)$. The Q-value is defined as: 
$Q=\left(\frac{R Q D}{J_{n}}\right) x\left(\frac{J_{r}}{J_{a}}\right) x\left(\frac{J_{W}}{S R F}\right)$

The first quotient $\left(R Q D / J_{n}\right)$ represents the overall structure of the rock mass and block size in the rock mass. The second quotient $\left(J_{r} / J_{a}\right)$ represents the shear strength of the discontinuity between two blocks ("inter-block shear strength"). The third quotient $\left(J_{w} / S R F\right)$ is related to the effective stress between the rock blocks. The descriptions and ratings for the various parameters are provided in extensive tables by Barton et al. (1974). The inter-block friction angle of the shear strength of a discontinuity between two blocks equals:

$\varphi_{\text {inter-block }}=\tan ^{-1}\left(\frac{J_{r}}{J_{a}}\right)$

\subsubsection{Sliding criterion}

The "sliding criterion" (Equation 5.3) was developed in the context of a classification system for rock slope failure (Hack \& Price 1995; Hack et al., 2003). The factors in the sliding criterion are partially based partially on earlier work by Laubscher (1990). The condition of a discontinuity is related to the maximum slope angle of a shear plane $\left(\varphi_{\text {sliding }}\right)$ in a rock mass, that sustains a stable rock block. The principle is similar to the "tilt-test" but on a larger scale (Price et al., 2009).

$\varphi_{\text {sliding }}$ (in degrees) $=(R l \times R s \times \operatorname{Im} \times K a) / 0.0113$

The factors $R /$ and $R s$ are related to the large- and small-scale roughness and material friction of the discontinuity, Im is a factor for the infill material, and $K a$ for the presence of karst along the discontinuity. The $R s$ and $R I$ are estimated based on visual comparison of standard curves with the trace of the discontinuity and on tactile roughness (i.e. "feeling by fingers"). The curves and tables for determining the values of the factors in Equation 5.3 are provided in Hack (1998); Hack et al. (2003).

\subsection{Iron precipitation}

The effect of iron precipitation in soil masses is well known from the formation of "hardened layers", "iron pans", and "iron duricrusts" in lateritic soils (Anon, 1990; Beauvais \& Colin, 1993; Chesworth, 2008). In rock masses, iron is often present in rock-forming minerals, in staining on mineral surfaces, and as an infill material in various forms in rock discontinuities. The mechanism of iron precipitation in discontinuities in a rock mass is controlled by water and gases dissolved in water, i.e. carbon dioxide and oxygen. Other processes in rock masses near surface due to weathering are hydration, solution, oxidation, and hydrolysis. Carbon dioxide $\left(\mathrm{CO}_{2}\right)$ dissolved in water decreases the $\mathrm{pH}$ of the water, which significantly influences its capacity to 
dissolve minerals, such as calcite. Oxygen $\left(\mathrm{O}_{2}\right)$ dissolved in water acts as oxidizing agent.

In higher elevated areas, the sequential interaction between groundwater movement and rock masses of sedimentary origin results in development of four basic weathering zones: "zone I; surface oxidation", "zone II; oxidation", "zone III; dissolved", and "zone IV; slightly weathered and fresh rock" (see below) (Chigira, 1990; Chigira \& Oyama, 2000; and Chigira \& Sone, 1991). In the oxidation zone, rock-forming minerals such as silicates and pyrite $\left(\mathrm{FeS}_{2}\right)$ are oxidized. Oxidation of pyrites produces sulphuric acid $\left(\mathrm{H}_{2} \mathrm{SO}_{4}\right)$ and releases iron as free ions that can precipitate as hydroxide $\mathrm{Fe}(\mathrm{OH})_{3}$ (Equation 5.4) (e.g. McKibben \& Barnes, 1986; Nicholson et al., 1988; Rimstidt \& Vaughan, 2003; Sasaki et al., 1998).

$4 \mathrm{FeS}_{2}+14 \mathrm{O}_{2}+4 \mathrm{H}_{2} \mathrm{O} \rightarrow 4 \mathrm{Fe}^{2+}+8 \mathrm{SO}_{4}^{2-}$

Dissolved $\mathrm{Fe}^{2+}$ oxidizes with dissolved oxygen in water to form $\mathrm{Fe}^{3+}$, which is very poorly soluble and precipitates as $\mathrm{Fe}(\mathrm{OH})_{3}$ or in the form of other hydroxides along the discontinuities in the mass or on the surface of rock forming mineral grains. If no sufficient oxygen is present, the sulphuric acid can further migrate through the rock mass downwards, dissolving rock forming minerals and cementing materials. This process will result in the development of the "dissolved" zone which is characterized by low rock density and higher porosity compared to the fresh rock. The increased acidity also enhances the formation of secondary clay minerals by weathering of feldspars, mica's, and other silicates, which may become the infill material in the discontinuities. The simple oxidizing mechanism described here may be more complex in reality for which is referred to the literature (Bland \& Rolls 1998).

\subsection{Methodology}

This study is part of research on weathering of cut slopes in a tropical environment and the data collected during fieldwork are organized via a systematic approach following the SSPC methodology (Hack et al., 2003). Details on the methodology applied in this research are given in Section 1.6. Special attention is given to the presence of iron in discontinuities, its characteristics, appearance, fabric, and position with respect to the weathering profile and grade. The characterisation of weathering of intact material and rock mass following ISO 14689-1:2003 (see Table 3.1) may not be optimum (Hencher, 2008; Price et al., 2009), but it is widely available and used, and therefore applied in this research. Apart from the description in the field, chemical analyses were done on samples of intact rock, discontinuity coatings, and infill material (see Section 5.6.1). 


\subsection{Weathering zones}

The thickness of the weathering profile in the research area varies and ranges from 3 to more than $30 \mathrm{~m}$ (Figure 5.1). Visually, the most obvious feature that distinguishes the zones is the colour of weathered material. The rock material in the upper part of the weathering profile is coloured brownish to yellowish (weathering zone I and part of zone II) while the lower part is mostly grey to greyish (zone III).

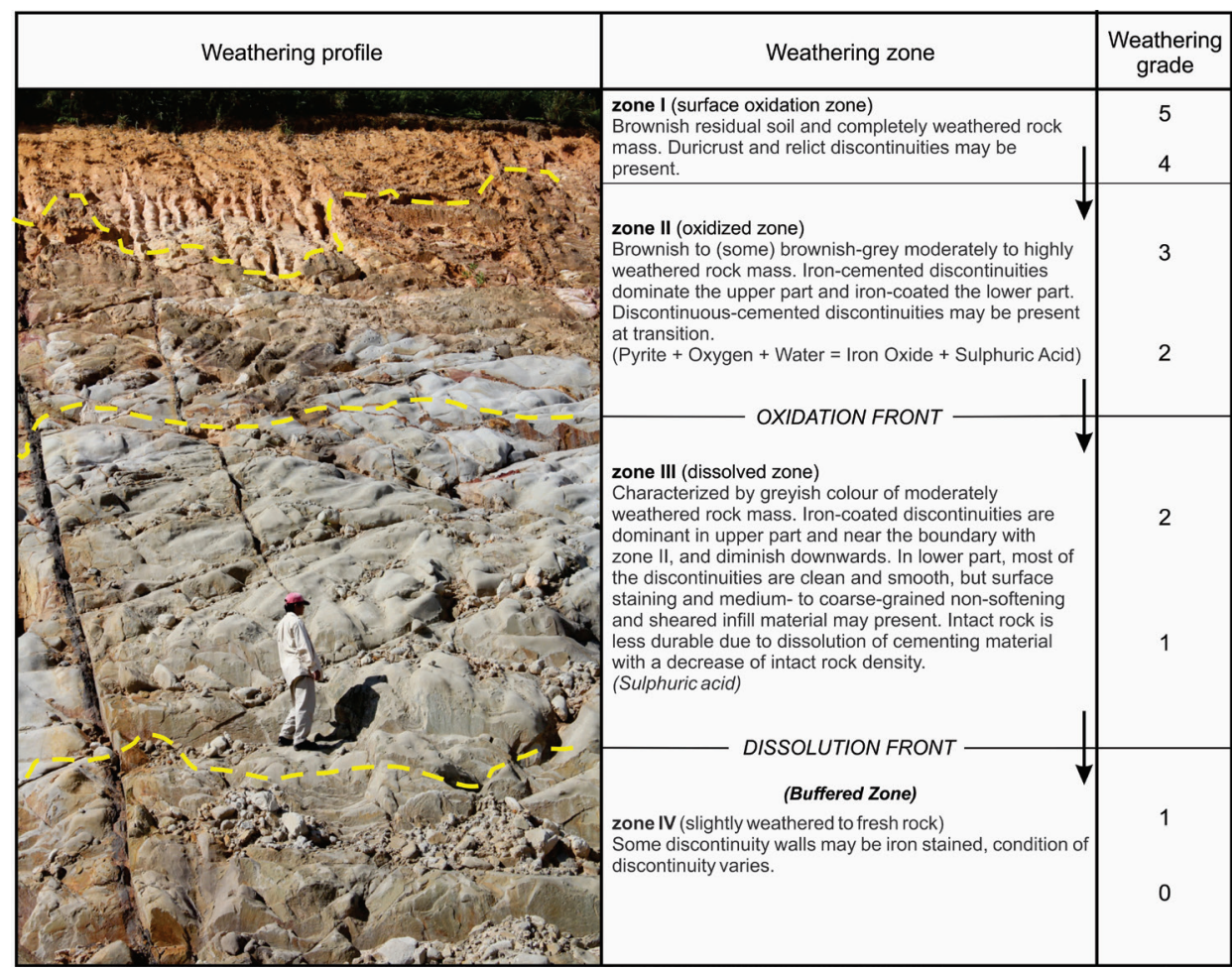

Figure 5.1 Typical weathering profile in sandstone in the study area. The boundaries between the zones are gradual. The arrows indicate the direction of flow of solution products; weathering grade follows (ISO 14689-1: 2003).

\subsubsection{Weathering zone I ("surface oxidation")}

Weathering zone I ("surface oxidation") is the upper part of the weathering profile and consists mainly of yellowish to reddish-brown residual soil (rock mass weathering grade 5) and completely weathered rock mass (grade 4), i.e. this zone consists of soil-type material. Relict iron coated or cemented discontinuities are common. Dark soil rich in organic material may be present too. The thickness of the zone ranges from 0.2 to $5 \mathrm{~m}$. The dominant chemical weathering process within this zone is oxidation by carbon dioxide originating from the atmosphere and biological activity (Chigira \& Sone, 
1991). Iron pans or duricrusts are also often present within this zone due to cementation by iron and alumina hydroxides.

\subsubsection{Weathering zone II ("oxidation")}

Weathering zone II ("oxidation") is characterized by brownish to yellowish intact rock and is composed of highly (grade 3) to moderately (grade 2) weathered rock mass. The thickness of the zone ranges from 0.5 to $6 \mathrm{~m}$. This zone is distinguished from weathering zone I by the soil-rock ratio, i.e. weathering zone II consists mainly of rock-type material while zone I mainly of soil-type. The dominant chemical weathering process in this zone is oxidation. Oxidation of intact rock minerals especially pyrites, produces iron oxide/hydroxide $\left(\mathrm{Fe}_{2} \mathrm{O}_{3} / \mathrm{Fe}(\mathrm{OH})_{3}\right)$ that precipitates along the discontinuities as well as between grain boundaries (Figure 5.2). These iron minerals are more resistant to later weathering and erosion than the intact rock, and causes the discontinuities to become extruding from the surface of the rock mass with progressing weathering and erosion.

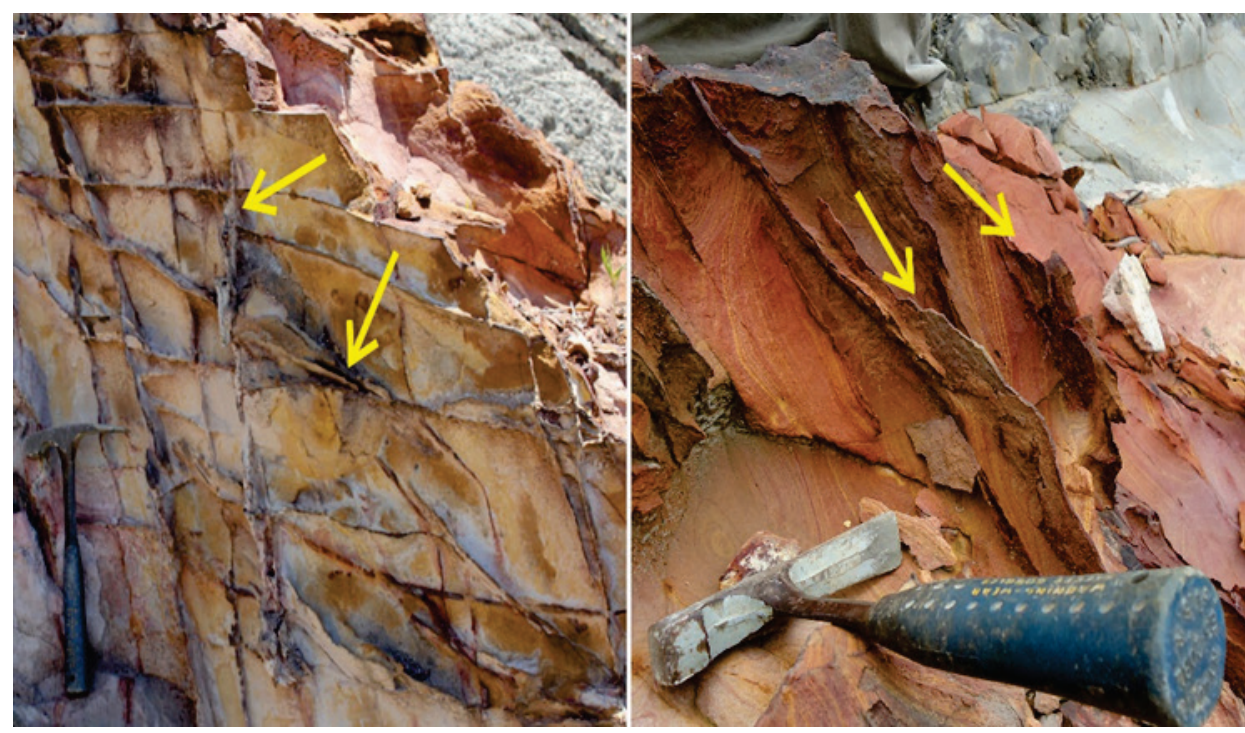

Figure 5.2 Relict iron-precipitated discontinuities in sandstone; the arrows indicate examples of relict discontinuities extruding up to about $5 \mathrm{~cm}$ from the rock mass surface (length of hammer $\approx 0.3 \mathrm{~m}$ ).

\subsubsection{Weathering zone III (“dissolution”)}

Weathering zone III ("dissolution") is characterized by greyish intact rock. This zone is composed of moderately (grade 2) to slightly (grade 1) weathered rock mass. The thickness of the zone varies from 2 to more than 7 $\mathrm{m}$. This zone is formed by the dissolution and leaching of rock forming minerals through the acidic solution percolating through from the oxidation 
zone above (i.e. weathering zone II). Iron precipitation along the discontinuities is rare and mostly only surface staining is present. Most of the discontinuities are smooth, and medium- to coarse-grained non-softening infill is often present. The boundary between zones II and III is the "oxidation front" however; the boundary is not marked by a sharp contrast but rather by a gradual transition, marked by a gradual change of colour from brownish to greyish. Weathering zone III may also show changes in the character of iron precipitation; from iron-cemented discontinuities in the top to iron-coated discontinuities towards zone IV. The thickness of the dissolution zone depends on the timespan dissolution occurred, drainage conditions, and the amount of buffer minerals such as calcite (Chigira, 1990).

\subsubsection{Weathering zone IV ("slightly weathered and fresh rock")}

In weathering zone IV ("slightly weathered and fresh rock"), the rock mass is fresh (grade 0 ) or only slightly affected by weathering (grade 1 ). The rock material has still its original colour (which is bluish grey for the SST unit); however, iron staining of discontinuity walls is often present. This zone is distinguished from the dissolution zone above by gradual changes in rock strength and density. The transition is denoted the "dissolution front" above which rock-forming minerals are leached and dissolved by the acidic solution derived from the oxidation zone. The dissolution front moves downward with time.

\subsection{Iron precipitation characteristics}

Iron precipitation in the discontinuities is divided into three types; stained, coated, and cemented (Figure 5.3). Iron staining is often observed on discontinuity surface of fresh to slightly weathered rock. It will caused discoloration on the rock surface without exert much changes on the shear strength of the discontinuity. Iron coating is referring to the formation of thin layer $(0.5-2 \mathrm{~mm})$ of iron oxide/hydroxide either on one side or both side of the opposing joints surface along discontinuity surface. These features are often observed in discontinuity within the oxidized zone. Some iron oxide "bridges" may be developed. Cemented joint is referring to the welded or healed of discontinuity. Iron precipitation is completely filling and cementing the discontinuity on both opposing discontinuity surfaces. The cemented joint thickness is varied and ranges from 0.5 to more than $5 \mathrm{~mm}$. These features are often observed within the oxidation zone in moderately to highly weathered rock. 


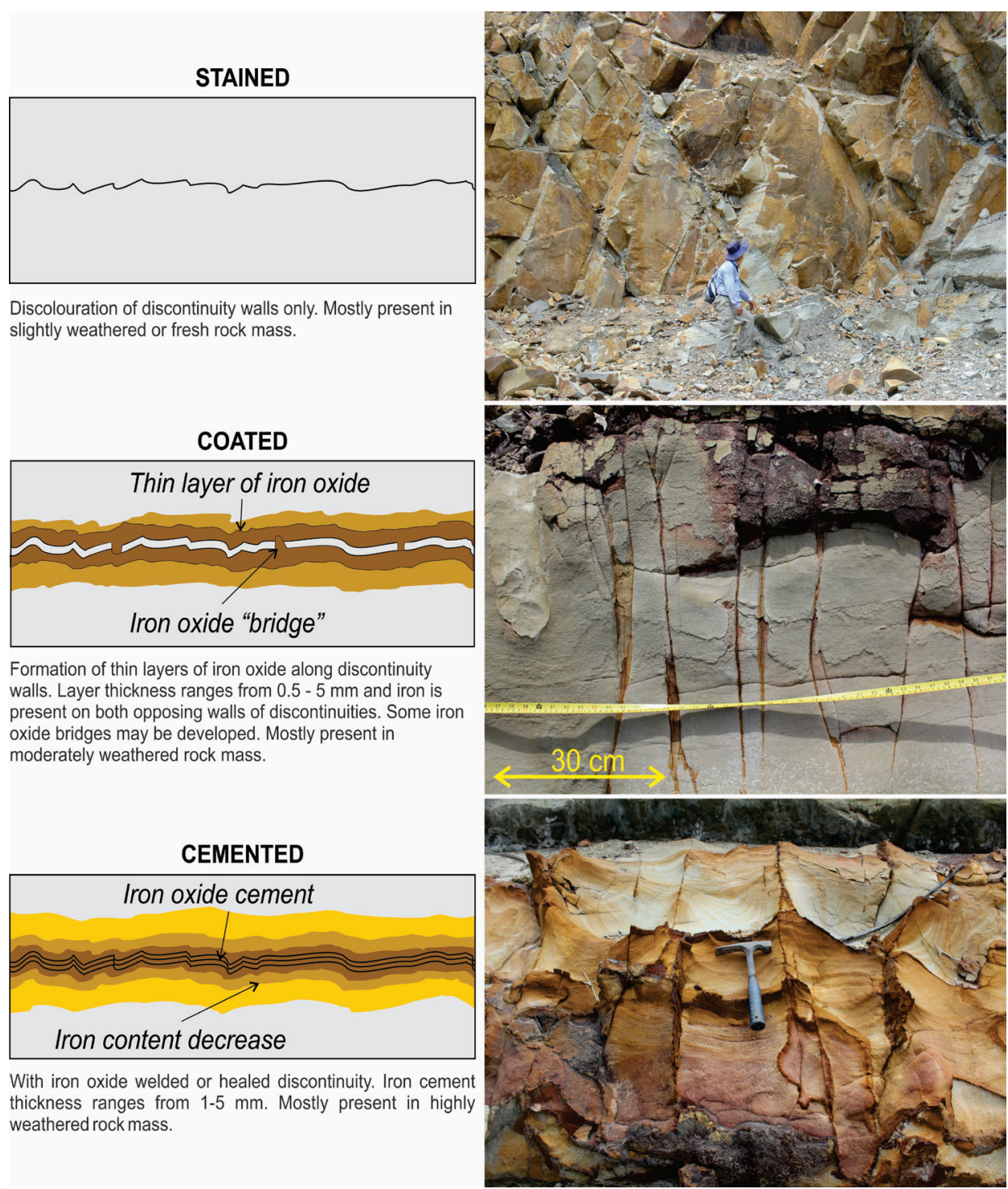

Figure 5.3 Iron precipitation types in discontinuities.

\subsubsection{Chemical analyses}

Intact rock samples from each weathering grade and zone, and samples of precipitated material in discontinuities were collected to determine the major element concentrations and in particular the iron content. Samples were analysed with a portable X-ray fluorescence (XRF) instrument (Niton XL3t, 2013) in a laboratory set-up with helium purging. The duration of the XRF measurements is about 85 seconds per sample with filter settings specifically 
to determine rock-forming elements. The dry rock densities were determined after the XRF analyses.

\subsubsection{Intact rock}

Iron precipitation occurs in between the rock forming material grains in intact rock (re-) cementing the grains, thus strengthening the intact rock. However, the degree of cementation gradually decreases from the origin of the cementation source (Figure 5.4). A high degree of cementation is related to a high concentration of iron on short distance, which normally occurs along discontinuities. The colour indicates the concentration of iron from brownish (high concentration) to greyish (low concentration). An oxidation front consisting of parallel lines of lighter and darker colour indicates an oscillating concentration of iron, which are likely formed by the same processes as socalled "Liesegang Rings" (Liesegang, 1896). Liesegang Rings are due to an interaction of reaction, precipitation, and diffusion, and are still subject of research (Scheel, 2009). The effect of increased strength and higher erosion resistivity due to iron precipitation is shown in Figure 5.4a-b in which the brownish areas are less susceptible to deterioration compared to the greyish areas (Figure $\mathbf{5 . 4 c - d ) . ~}$
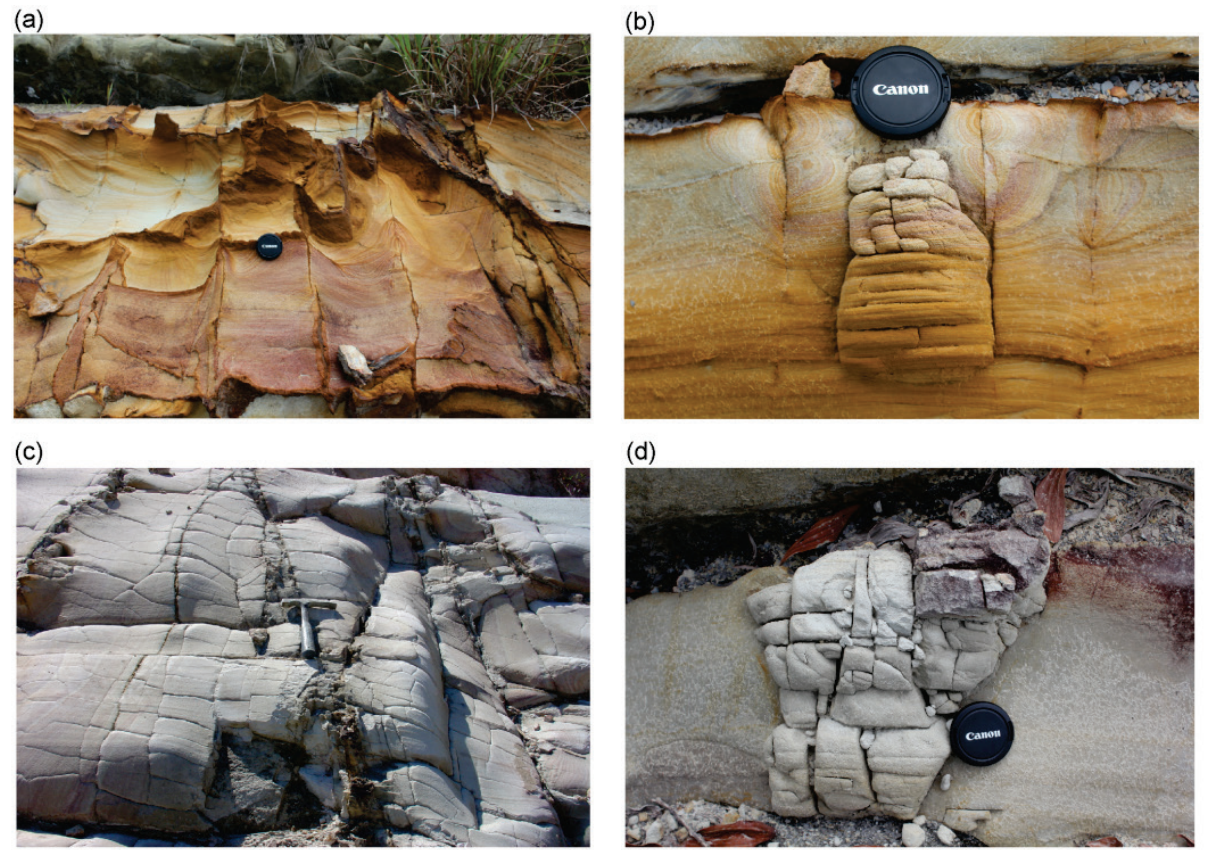

Figure 5.4 Development of discontinuities in oxidation zone (a-b) and dissolution zone (c-d). The iron content gradually decreases towards the dissolution zone or inwards into the intact rock. 
Table 5.1 shows the result of XRF analyses for some major elements in sandstone. The main element is silica $(S i)$ as expected. If all sulphur content is assumed to be in the form pyrite $\left(\mathrm{FeS}_{2}\right)$, pyrite forms 0.04 to $2.04 \%$ of the constituents heterogeneously distributed throughout the rock mass.

Table 5.1 Result of the X-ray fluorescence (XRF) analysis.

\begin{tabular}{|c|c|c|c|c|c|c|c|c|c|c|}
\hline \multirow[t]{2}{*}{ Sample } & \multirow{2}{*}{$\begin{array}{l}\text { Rock mass } \\
\text { weathering } \\
\text { grade* }\end{array}$} & \multirow{2}{*}{$\begin{array}{l}\text { Weathering } \\
\text { zone** }\end{array}$} & \multicolumn{8}{|c|}{ Mineral content (\%) } \\
\hline & & & $\mathrm{Si}+$ & $\mathrm{Ca}$ & $\mathrm{Fe}$ & $\mathrm{Al}$ & $\mathrm{K}$ & $\mathrm{Mg}$ & $\mathrm{S}$ & $\mathrm{Ti}$ \\
\hline KK65 & 0 & IV & 40.39 & 1.26 & 1.19 & 2.52 & 0.75 & 0.18 & 0.02 & 0.12 \\
\hline KK83 & 0 & IV & 39.59 & 1.26 & 1.39 & 3.46 & 1.73 & 0.42 & 0.08 & 0.13 \\
\hline KK56 & 0 & IV & 44.11 & 0.02 & 0.96 & 2.44 & 0.8 & 0.24 & n.d. $\neq$ & 0.16 \\
\hline KK78 & 1 & III & 40.86 & 0.03 & 1.43 & 2.93 & 0.91 & 0.31 & 0.03 & 0.13 \\
\hline KK86 & 1 & III & 37.91 & 0.04 & 1.48 & 3.63 & 1.15 & 0.47 & 0.04 & 0.18 \\
\hline KK81a & 2 & II & 33.61 & 0.19 & 1.62 & 3.58 & 1.46 & 0.50 & 1.09 & 0.15 \\
\hline KK39 & 2 & III & 36.37 & 0.03 & 0.99 & 3.09 & 1.08 & 0.29 & 0.08 & 0.13 \\
\hline KK88 & 2 & III & 37.32 & 0.06 & 1.08 & 3.54 & 1.45 & 0.33 & 0.22 & 0.13 \\
\hline KK81b & 2 & II & 34.73 & 0.08 & 2.53 & 3.92 & 1.39 & 0.40 & 0.15 & 0.15 \\
\hline KK15 & 3 & II & 36.31 & 0.04 & 1.75 & 4.48 & 1.17 & 0.52 & 0.08 & 0.15 \\
\hline R1 & 3 & I & 31.96 & 0.1 & 2.86 & 4.87 & 1.50 & 0.46 & 0.25 & 0.16 \\
\hline R46 & 3 & I & 35.89 & n.d & 0.80 & 3.68 & 1.19 & 0.20 & $<0.01$ & 0.16 \\
\hline
\end{tabular}

Calculated as $\mathrm{SiO} 2$ (quartz); total quartz content ranges between 68 and $94 \%$. $\neq$.d. = not detected.

Table 5.2 and Figure $\mathbf{5 . 5}$ show the chemical composition and density of the intact rock per rock mass weathering grade. In general, it shows that the amounts of $\mathrm{SiO}_{2}$ and $\mathrm{CaCO}_{3}$ are decreasing with higher weathering grades. This may be attributed to the dissolution processes by acidic solution in the dissolved zone.

Table 5.2 Major average chemical elements in intact rock per rock mass weathering grade.

\begin{tabular}{|c|c|c|c|c|c|c|c|c|c|}
\hline \multirow{2}{*}{$\begin{array}{l}\text { Weathering } \\
\text { grade }^{*}\end{array}$} & \multirow{2}{*}{$\begin{array}{l}\text { Density } \\
\left(\mathrm{kg} / \mathrm{m}^{3}\right)\end{array}$} & \multicolumn{8}{|c|}{ Major element content (\%) } \\
\hline & & $\mathrm{SiO}_{2}$ & $\mathrm{CaCO}_{3}$ & $\mathrm{Al}_{2} \mathrm{O}_{3}$ & $\mathrm{~K}_{2} \mathrm{O}$ & $\mathrm{MgO}$ & $\mathrm{Fe}_{2} \mathrm{O}_{3}$ & $\mathrm{FeO}$ & $\mathrm{TiO}_{2}$ \\
\hline 0 & 2,605 & 88.48 & 2.12 & 5.30 & 1.15 & 0.47 & 1.69 & 1.52 & 0.23 \\
\hline 1 & 2,484 & 87.61 & 0.09 & 5.67 & 1.00 & 0.57 & 1.85 & 1.66 & 0.26 \\
\hline 2 & 2,441 & 75.95 & 0.23 & 6.67 & 1.41 & 0.62 & 2.23 & 2.00 & 0.24 \\
\hline 3 & 2,383 & 74.26 & 0.11 & 8.21 & 1.35 & 0.65 & 2.58 & 2.32 & 0.26 \\
\hline
\end{tabular}

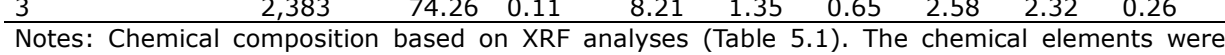
converted into elements oxide and carbonate by multiplying with specific element oxide or carbonate conversion factors. "Average" denotes the average chemical content from different samples with the same weathering grade. Three samples were used to determine the average chemical contents for each weathering grade, except for weathering grade 3 for which only 2 samples were used. * Rock mass weathering grade follow Table 3.1.** Weathering zones based on Figure 5.1. 


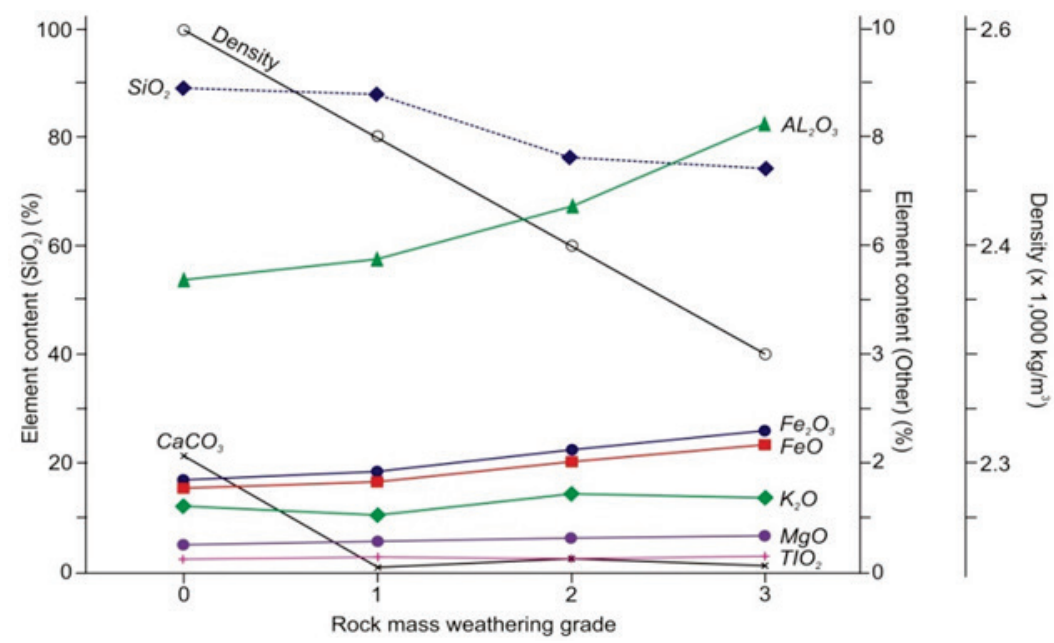

Figure 5.5 Average chemical element content and density vs. rock mass weathering grade; $\mathrm{SiO}_{2}$ and $\mathrm{CaCO}_{3}$ content decrease, while $\mathrm{Al} 2 \mathrm{O} 3$ and $\mathrm{Fe}$ increase with higher grades of weathering. $\mathrm{SiO}_{2}$ content on left axis; contents of other elements on right axis.

\subsubsection{Discontinuities}

Table 5.3 shows the iron concentration in discontinuities for both iron oxide $\mathrm{FeO}$ and iron hydroxide $\left(\mathrm{Fe}_{2} \mathrm{O}_{3}\right)$. As expected the iron content increases from stained to cemented.

Table 5.3 Iron content in the form of $\mathrm{Fe}_{2} \mathrm{O}$ and $\mathrm{Fe}_{2} \mathrm{O}_{3}$ per precipitation type in discontinuities (average in brackets).

\begin{tabular}{llll}
\hline $\begin{array}{l}\text { Iron precipitation } \\
\text { type }\end{array}$ & $\mathrm{FeO}(\%)$ & $\mathrm{Fe}_{2} \mathrm{O}_{3}(\%)$ & Total Fe (\%) \\
\hline Stained & $1.4-3.8(2.4)$ & $1.5-4.2(2.7)$ & $1.1-2.9(1.9)$ \\
Coated & $3.9-12.5(7.1)$ & $4.3-13.9(7.9)$ & $3.3-9.7(5.1)$ \\
Cemented & $20.2-30.6(25.6)$ & $22.4-34(28.5)$ & $15.7-23.8(19.9)$ \\
\hline
\end{tabular}

\subsection{Iron precipitation effect on shear strength}

The effect of iron precipitation in discontinuities on the shear strength is shown for different shear strength relations for discontinuities. For all discontinuities is assumed that the condition of the discontinuity visible at the exposure is similar to the condition deeper inside the rock mass, which is very likely because the exposures were excavated quite recent before the research.

\subsection{1 $\varphi_{\text {inter-block }}$}

The effect of iron precipitation is incorporated by assigning appropriate values for $J_{r}$ and $J_{a}$ based on the descriptions given by Barton et al. (1974) (Table 5.4). A $J_{r}$ with a value of 3 can also include the presence of iron-bridges 
along discontinuities. The $\varphi_{\text {inter-block }}$ shows that the friction angle of ironcemented discontinuities is higher compared to iron-coated and stained discontinuities (Figure 5.6).

Table 5.4 Jr and Ja values for iron-precipitated discontinuities with pinter-block. Jr and Ja values and descriptions following Barton et al. (1974).

\begin{tabular}{|c|c|c|c|c|c|}
\hline \multirow{2}{*}{$\begin{array}{l}\text { Iron } \\
\text { precipitation } \\
\text { type }\end{array}$} & \multicolumn{2}{|l|}{$J r$} & \multicolumn{2}{|l|}{ Ja } & \multirow{2}{*}{$\begin{array}{l}\varphi_{\text {inter-block }} \\
\text { (degrees) }\end{array}$} \\
\hline & Value & Description & Value & Description & \\
\hline \multirow[t]{2}{*}{ Cemented } & 4 & $\begin{array}{l}\text { Discontinuous } \\
\text { joint }\end{array}$ & 0.75 & $\begin{array}{l}\text { Tightly healed, hard, non- } \\
\text { softening, impermeable filling }\end{array}$ & 79.4 \\
\hline & 3 & $\begin{array}{l}\text { Rough or } \\
\text { irregular, } \\
\text { undulating }\end{array}$ & 3 & $\begin{array}{l}\text { Slightly altered joint walls, non- } \\
\text { softening mineral coatings } \\
\text { Silty-or sandy clay coating, } \\
\text { small clay-fraction (non- } \\
\text { softening) }\end{array}$ & 56.3 \\
\hline \multirow{7}{*}{ Coated } & \multirow[b]{2}{*}{1.5} & \multirow{2}{*}{$\begin{array}{l}\text { Rough or } \\
\text { irregular, planar }\end{array}$} & 2 & $\begin{array}{l}\text { Slightly altered joint walls, non- } \\
\text { softening mineral coatings }\end{array}$ & 36.9 \\
\hline & & & 3 & $\begin{array}{l}\text { Silty-or sandy clay coating, } \\
\text { small clay-fraction (non- } \\
\text { softening) }\end{array}$ & 26.6 \\
\hline & \multirow[b]{2}{*}{3} & \multirow{2}{*}{$\begin{array}{l}\text { Rough or } \\
\text { irregular, } \\
\text { undulating }\end{array}$} & 1 & $\begin{array}{l}\text { Unaltered joint walls, surface } \\
\text { staining only }\end{array}$ & 71.6 \\
\hline & & & 3 & $\begin{array}{l}\text { Silty-or sandy clay coating, } \\
\text { small clay-fraction (non- } \\
\text { softening) }\end{array}$ & 45 \\
\hline & \multirow[b]{2}{*}{2} & \multirow{2}{*}{$\begin{array}{l}\text { Smooth } \\
\text { undulating }\end{array}$} & 1 & $\begin{array}{l}\text { Unaltered joint walls, surface } \\
\text { staining only }\end{array}$ & 63.4 \\
\hline & & & 3 & $\begin{array}{l}\text { Silty-or sandy clay coating, } \\
\text { small clay-fraction (non- } \\
\text { softening) }\end{array}$ & 33.7 \\
\hline & \multirow[b]{2}{*}{1.5} & \multirow{2}{*}{$\begin{array}{l}\text { Rough or } \\
\text { irregular, planar }\end{array}$} & 1 & $\begin{array}{l}\text { Unaltered joint walls, surface } \\
\text { staining only }\end{array}$ & 56.3 \\
\hline \multirow[t]{5}{*}{ Stained } & & & 3 & $\begin{array}{l}\text { Silty-or sandy clay coating, } \\
\text { small clay-fraction (non- } \\
\text { softening) }\end{array}$ & 21.8 \\
\hline & \multirow[b]{2}{*}{1} & \multirow[b]{2}{*}{ Smooth planar } & 1 & $\begin{array}{l}\text { Unaltered joint walls, surface } \\
\text { staining only }\end{array}$ & 45.0 \\
\hline & & & 3 & $\begin{array}{l}\text { Silty-or sandy clay coating, } \\
\text { small clay-fraction (non- } \\
\text { softening) }\end{array}$ & 18.4 \\
\hline & \multirow[b]{2}{*}{0.5} & \multirow{2}{*}{$\begin{array}{l}\text { Slickensided } \\
\text { planar }\end{array}$} & 1 & $\begin{array}{l}\text { Unaltered joint walls, surface } \\
\text { staining only }\end{array}$ & 26.6 \\
\hline & & & 3 & $\begin{array}{l}\text { Silty-or sandy clay coating, } \\
\text { small clay-fraction (non- } \\
\text { softening) }\end{array}$ & 9.5 \\
\hline
\end{tabular}

\subsection{2 $\varphi_{\text {sliding }}$}

The $\varphi_{\text {sliding }}$ (see section 5.2.2) does not incorporate a specific value for ironprecipitated discontinuities. However, the presence of iron can be simulated by adjusting the values for the small-scale roughness (Rs) and infill material (Im). The values for Rs and Im recommended for cemented discontinuities are 0.95 (rough stepped) and 1.07 (cemented infill). For coated discontinuity surfaces, an Rs value of 0.95 (rough stepped) is to be used, while the Im value is dependent on the actual infill, which varies per discontinuity, and the value is thus based on field observations. The contribution of iron bridges in 
coated discontinuities is difficult to access as these may vary all over the discontinuity plane also inside in the rock mass which is invisible. Therefore, the sliding angle is calculated without incorporating the contribution of iron bridges. The resulting sliding angle should therefore be regarded as a minimum value. The value for the condition of a discontinuity for stained discontinuities is based on field observations. The results show that the presence of iron in coated and cemented discontinuities significantly increases the sliding angle of the discontinuities (Figure 5.6).

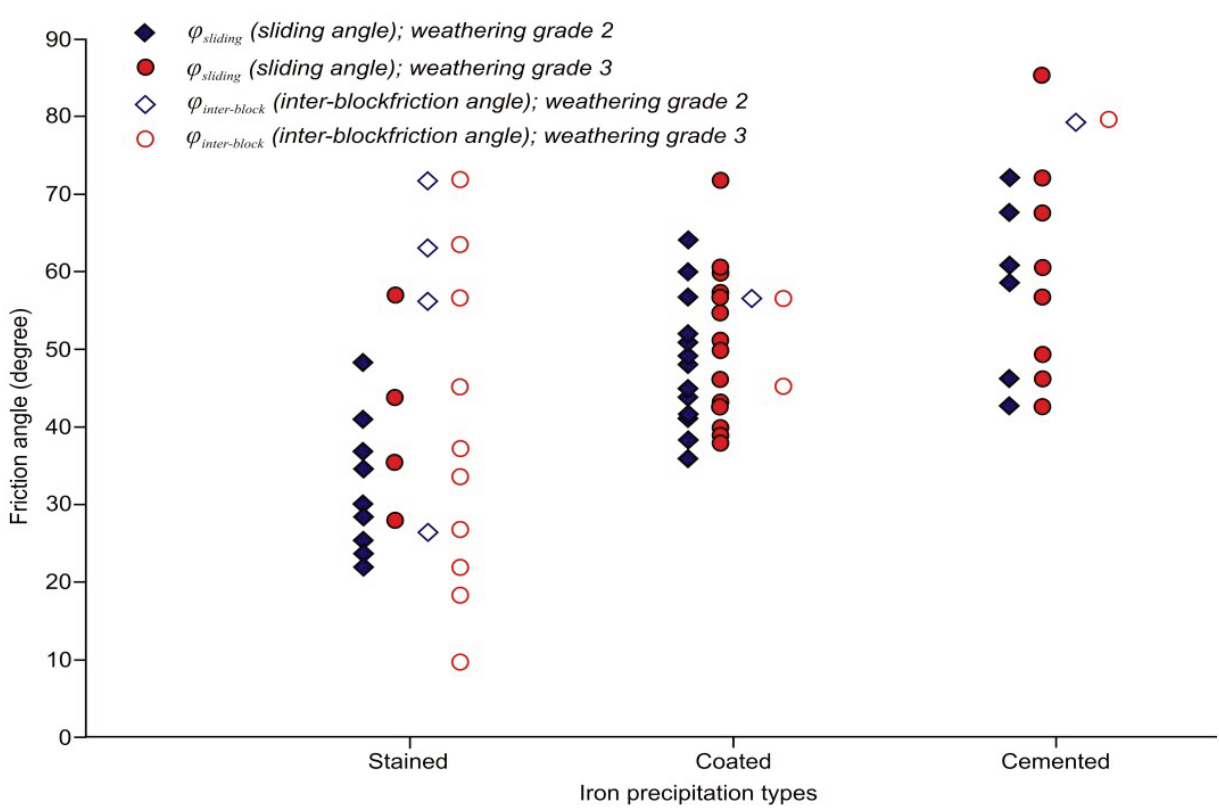

Figure 5.6 Influence of different types of iron precipitation on the sliding angle (Equation 5.3) and inter-block friction angle (Equation 5.2) of discontinuities for moderately (grade 2 ) and highly (grade 3 ) weathered rock masses.

\subsubsection{Slope stability}

Visual observation and calculation of the stability of slopes show that rock exposures with iron-precipitated discontinuities are less susceptible to slope instability compared to similar rock exposures without (Figure 5.7). Even if the weathering grade increases, the relict iron-precipitated discontinuities give a reinforcing effect of the rock mass forming the slope. The susceptibility to weathering reduces as well because erosion of the rock mass surface is reduced due to the presence of the extruding discontinuities. A reduction of erosion allows a thicker layer of weathered material to act as protection of the underlying material against weathering (Huisman et al., 2006). 


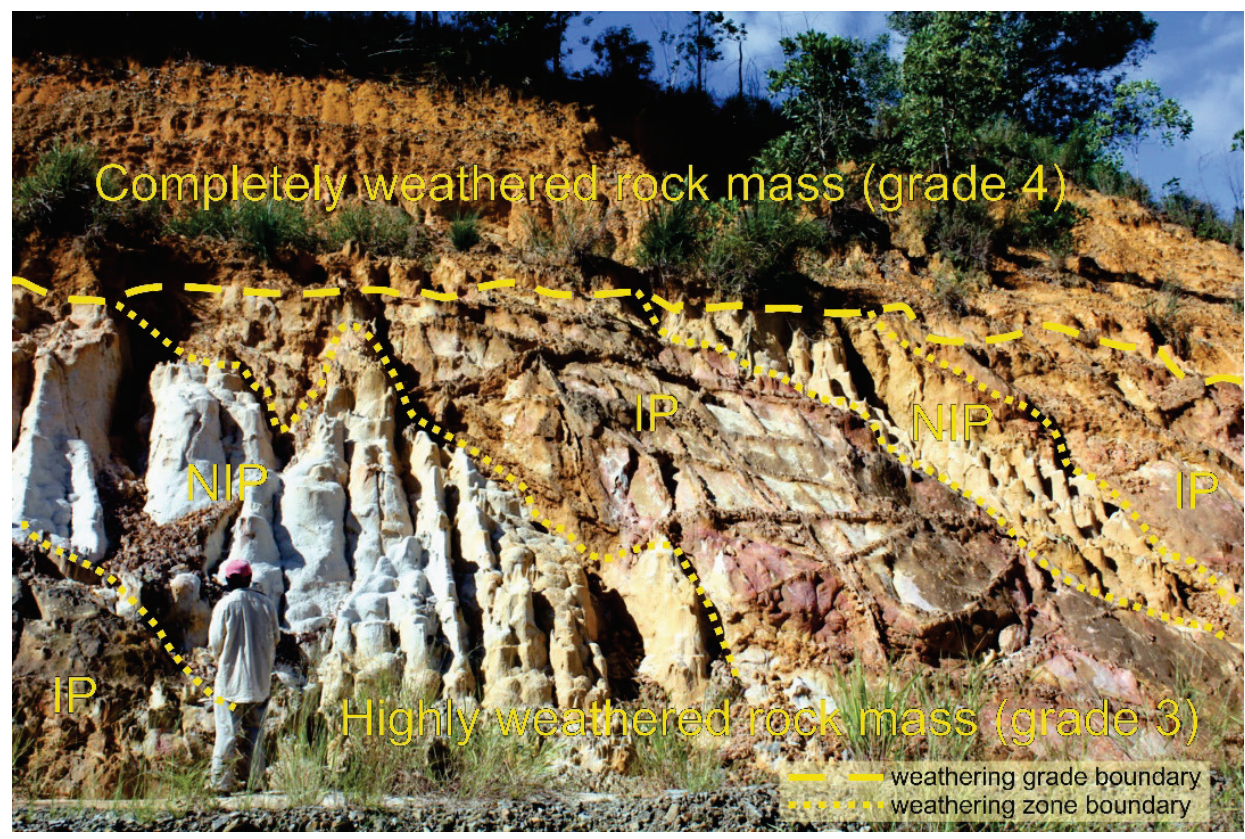

Figure 5.7 Iron precipitation in discontinuities reinforces the stability of a cut slope; susceptibility to slope degradation is low in rock mass with iron-precipitated discontinuities (IP) compared to rock mass without (NIP).

\subsection{Discussion}

Weathering zones and its association with particular types of iron precipitation have a significant influence on the properties of discontinuities and rock masses (Table 5.5). Iron precipitation increases the shear strength of discontinuities by stronger and harder discontinuity walls, more rough walls, and by forming bridges or continuous cemented infill as shown by the inter-block and sliding friction in Figure 5.6. This is consistent with the analysis by Hencher \& McNicholl (1995) that shows that iron precipitation on discontinuity walls is rarely associated with a low friction angle. Iron precipitation also leads to a partial or complete recovery of the mechanical properties of previously weathered intact rock, discontinuities, and rock mass, as reported by Cuccuru et al. (2012) and Fell et al. (2012).

Iron precipitation may also have mechanical effects depending on differences in intact rock texture. In the oxidation zone coarse-grained intact rock tends to be strengthened due to grain cementation whereas fine-grained rock becomes more susceptible to further deterioration (Chigira \& Oyama, 2000). In the dissolution zone, cement between grains such as calcite and siliceous minerals, is dissolved resulting in more friable intact rock (Figure 5.7). 
The characteristics of weathering zones in Kota Kinabalu, Malaysia found in the present research are similar to those reported for sedimentary rock in Japan (Chigira, 1990; Chigira \& Oyama, 2000). Both are developed in high elevation or hilly areas in which the migration of water, oxygen fluxes, and acidic solution are downwards through the rock mass to a lower elevation. This results in a sequence of distinctively separated zones of oxidation and dissolution following the direction of fluid flow. In flatter areas the fluid flows are more mixed reducing the effect of differentiated zones (Chigira \& Oyama, 2000). The sequence may not always be due vertical as local differences in morphology and permeability of the rock mass, e.g. faults or fracture zones, may give preferred flow directions that are not vertically down. See for example Figure 5.7, where the different zones are inclined to the right.

Table 5.5 Summary of characteristics of iron precipitation in sandstone rock mass.

\begin{tabular}{|c|c|c|c|c|c|c|}
\hline \multirow{2}{*}{$\begin{array}{l}\text { Type of iron } \\
\text { precipitation }\end{array}$} & \multirow{2}{*}{$\begin{array}{l}\text { Weathering } \\
\text { zone }\end{array}$} & \multirow{2}{*}{$\begin{array}{l}\text { Rock mass } \\
\text { weathering*+ }\end{array}$} & \multirow[t]{2}{*}{ Description } & \multicolumn{2}{|c|}{ Iron content (\%) } & \multirow[t]{2}{*}{ Shear strength } \\
\hline & & & & Range & Average & \\
\hline \multicolumn{7}{|c|}{ Discontinuities } \\
\hline Stained & $\begin{array}{l}\text { Slightly to } \\
\text { fresh rock }\end{array}$ & $\begin{array}{l}\text { Fresh-slightly } \\
\text { (grades } 0 \text { \& } \\
\text { 1) }\end{array}$ & $\begin{array}{l}\text { Staining only } \\
\text { (discoloured } \\
\text { surfaces) }\end{array}$ & $1.1-2.9$ & 1.9 & $\begin{array}{l}\text { Controlled by } \\
\text { the roughness } \\
\text { of the } \\
\text { discontinuity } \\
\text { walls and infill } \\
\text { (if present) }\end{array}$ \\
\hline Coated & Oxidation & $\begin{array}{l}\text { Moderately- } \\
\text { high } \\
\text { (grades } 2 \& \\
\text { 3) }\end{array}$ & $\begin{array}{l}\text { Thin layers } \\
(0.5-2 \mathrm{~mm}) \text { of } \\
\text { iron oxide along } \\
\text { the } \\
\text { discontinuity } \\
\text { walls; may be } \\
\text { present on both } \\
\text { opposite } \\
\text { discontinuity } \\
\text { walls; locally } \\
\text { iron oxide } \\
\text { bridges }\end{array}$ & $3.3-9.7$ & 5.1 & $\begin{array}{l}\text { Increased due } \\
\text { to hardened and } \\
\text { rougher } \\
\text { discontinuity } \\
\text { walls and } \\
\text { bridges }\end{array}$ \\
\hline Cemented & Oxidation & $\begin{array}{l}\text { Moderately- } \\
\text { highly } \\
\text { (grades } 2 \& \\
\text { 3) }\end{array}$ & $\begin{array}{l}\text { Healed } \\
\text { discontinuities } \\
\text { or welded } \\
\text { opposing } \\
\text { discontinuity } \\
\text { walls }(0.5-> \\
5 \mathrm{~mm})\end{array}$ & $\begin{array}{l}18.8- \\
23.8\end{array}$ & 21.3 & $\begin{array}{l}\text { Equivalent to or } \\
\text { higher than the } \\
\text { surrounding } \\
\text { intact rock }\end{array}$ \\
\hline Intact rock & \multicolumn{6}{|c|}{$\begin{array}{l}\text { Intact rock blocks discoloured to brownish or rusty in appearance; iron precipitation } \\
\text { on and between rock forming material grains; iron concentration is high along the } \\
\text { discontinuity surfaces and gradually decreases inwards }\end{array}$} \\
\hline
\end{tabular}

The research was done on cut slopes that had been excavated for days to a couple of years before the investigation. The authors do not expect that the rock mass characteristics are seriously altered by weathering during the time of exposure. However, erosion certainly affected the surfaces as is shown by the extruding relict discontinuities (see Figure 5.2). 


\subsection{Conclusion}

The weathering profile of sedimentary sandstone rock masses in the area of Kota Kinabalu Sabah, Malaysia shows oxidation and dissolution of iron that governs the formation of weathering zones. Oxidation processes will cause iron precipitation in discontinuities as well as within intact rock, while in other zones iron is removed by dissolution. The presence or absence of secondary iron minerals has a marked influence on the properties of the rock mass and discontinuities.

Three types of iron precipitation in discontinuities have been identified in the weathering profile, i.e. stained, coated, and cemented. Iron coating and cementing are often observed in moderately to highly weathered rock whereas staining is often observed in slightly weathered and fresh rock masses. Susceptibility to weathering of sandstone with iron-precipitated discontinuities is reduced. In stability analyses the presences of weathering zones should probably be added to the description of the "condition of discontinuity" in rock mass descriptions and classification systems because, for example the susceptibility to weathering depends on the weathering zone. 


\section{Chapter 6: Landslide susceptibility analysis}

This chapter describes the assessment and selection of suitable landslide causal parameter groups in the analysis of a landslide susceptibility map (LSM) for the northern part of the Kota Kinabalu area. The assessment is based on the value of area under the curve (AUC) of "success rate curve". The LSM was developed based on the information value statistical method utilising a suitable combination of landslide causal parameter groups. This chapter is partly based on the article "Landslide susceptibility assessment using information value statistical method: a case study at northern Kota Kinabalu, Sabah Malaysia" (Tating et al., 2015c).

\subsection{Introduction}

Landslides are one of the most frequent types of natural hazard in the hilly and mountainous areas of Malaysia. They may lead to not only loss of life but also to large economic losses due to property damage and indirect effects such as disruption of the transportation network. The total economic losses due to landslides reported for a period of three decades from 1973 to 2007, is estimated to be about 3 billion Malaysian Ringgit (about 1billion US\$) (PWD, 2009). Landslide incidents and fatalities due to landslides have also increased during the same period. The increased numbers of landslides are mostly related to expansion of development into hilly areas because of increasing population, urbanization, deforestation, and development of infrastructure.

Disaster and economic losses due to landslide hazard can be minimized and avoided in landslide prone areas if information about the frequency, magnitude and the characteristics of landslides within a particular area are identified in advance. However, in some countries including Malaysia, identification and delineation of landslide prone areas has not been done, partly due to government policy that is favouring a post disaster management rather than pre-disaster management (PWD, 2009). Landslide disaster management is often misunderstood as merely providing relief to victims, aiding recovery following an event and rebuilding damaged infrastructure; as a result only limited resources and funds have been allocated for landslides hazard identification and risk management strategies in areas prone to landslides. Awareness regarding the economic and social impact of landslides begun after the collapse of Block 1 of the Highland Towers on 11 September 1993 which claimed 48 lives (Jamaludin and Hussien, 2006; Qasim et al., 2013). Since then, interest in landslide investigation and management increased, as shown by the establishment of the slope engineering branch under the Public Works Department (PWD) in 2004. In addition, geological terrain mapping is done by the Minerals and 
Geoscience Department (DMG) of Malaysia throughout the country to make thematic maps that can be used as guidance for advice to local councils regarding constructions on hilly terrain.

Identification of landslide prone areas through susceptibility assessment is essential in order to understand landslide phenomena and its relationship with various causative factors. Landslides occur as a combination of several factors which can be distinguished into preparatory factors (such as slope angle, geology, and land use) and triggering factors (such as rainfall and earthquakes). These factors can be utilized to develop a landslide susceptibility map, which delineates and ranks the slope stability in an area from low susceptibility to high susceptibility (Chacon et al., 2006). Susceptibility refers to the spatial future likelihood or probability for landslides to occur (Hervas and Bobrowsky, 2009).

There are many susceptibility mapping methods published in the literature which generally can be grouped into two broad methods; qualitative and quantitative methods. Qualitative methods rely on expert opinion and may be subjective. Qualitative methods are commonly organized by directly weighting (indexing) the influence of, for example, geological and geomorphological features on the landslide susceptibility level (Soeters and van Westen, 1996). Quantitative methods are based on a numerical expression of the relationship between causal factors and landslide occurrence. The latter can be divided into two main methods: deterministic and statistical (Aleotti and Chowdhury, 1999; Ayalew and Yamagishi, 2005).

These methods mainly use environmental factors (with slope angle as the main driver), land use, structural geology, material properties, and antecedent rainfall as more or less correlated factors. Some factors may be included depending on the area and scale of the analysis. Human-induced factors such as shifting cultivation are seldom included, except for distance to roads. Furthermore, the effect of deterioration processes in time (i.e. weathering) on the occurrence of landslides especially along roads and related to cut slopes are mostly not used in susceptibility analyses.

The main objective of the research is to assess the predictive power of several selected landslide causal parameter groups in the analysis of landslide susceptibility map (LSM) for the northern part of Kota Kinabalu. In this research, man-induced parameters are separated to investigate their influence with respect to the other groups, on a regional scale. This can be done by exclusion of each landslide causal parameter groups in turn during the determination of landslide susceptibility index (LSI) using Equation 6.1. Furthermore, it may help in further understanding the role of the landslide causal parameter groups in improving the LSM. In addition to the main 
objective, the research is also carried out to investigate if such a susceptibility map helps in developing a disaster preparedness policy for the northern part of Kota Kinabalu. The LSM is based on a statistical quantitative method; the Information Value method (Yin and Yan, 1988; Jade and Sarkar, 1993; Zezere, 2002; Yalcin, 2008). This method is also known as landslide index method (van Westen, 1997).

\subsection{Previous landslide assessment study in Kota Kinabalu, Sabah}

Only few landslide assessments in Kota Kinabalu, Sabah in East Malaysia have been done. Among the earliest studies is the study by Tongkul (1989) in which "weak zones" in the vicinity of Kota Kinabalu were characterized, delineated and their implications on future construction discussed. Tongkul (1989) classified weak zones as zones of disrupted/deformed strata (e.g. fault zones), which predominantly consist of various sizes of sedimentary blocks embedded in fine grain sedimentary materials such as shale and mudstone. These zones are the results of the tectonic activities in the past and their presence may relate to landslide incidents in natural slopes and slope failures in road cut slopes. However, landslides and slope failures are normally caused by set causal factors that can be analysed using several methods to delineate susceptibility to landslides.

About a decade later, Tating (2003) conducted a landslide inventory investigation in the northern part of Kota Kinabalu and related landslide occurrences with the lithology. The main controlling factors for landslides were determined based on a direct observation (heuristic) method coupled with extensive fieldwork. The result of the study shows that most of the landslides occur in areas underlain by interbedded sandstone and shale, and some are associated with weathered thick-bedded sandstone. Shallow translational landslides are the dominant type of landslides, especially in the sandstone and shale interbeds, whereas rotational landslides were mostly observed in thick-bedded sandstone. Landslide causative factors are mostly due to the disturbance of natural slopes by excavations or cut slopes, vegetation clearance for agricultural and residential purposes, and by weathering of the exposed rock.

Recent landslide assessments in the Kota Kinabalu area are carried out by Alvyn (2011) and Rodeano et al. (2011a, 2011b, 2012a \& 2012b) by using various methodologies. Alvyn (2011) carried out landslide assessment based on Probabilistic Frequency Ratio (PFR) model on a regional scale of $1: 50,000$ and concluded that the main causal factor for landslides are geomorphological factors (slope angle, slope aspect, soil types, followed by 
human factors (such as landuse and road construction) and geological factors (lithology, structural geology).

Rodeano et al. (2011a, 2011b, 2012a \& 2012b) compared various statistical methodologies for landslides assessment at a regional scale of $1: 50,000$. The results of the various landslide susceptibility maps show considerable differences. Seemingly different landslide assessment methods may produces different susceptibility maps, which makes the correctness of the used methodologies and resulting maps questionable. The differences may be attributed to an inappropriate selection of the assessment methodology, erroneous use of causal factors, and varying level of detail of landslide inventory data used for the regional scale assessment. Nevertheless, landslide susceptibility model validation based on the area under curve value $(A \cup C)$, which is about 0.75 , shows that the landslides susceptibility models are statistically acceptable. It should be noted that the assessment is based on regional scale surveys.

\subsection{Research methods}

\subsubsection{Landslide Inventory map}

In general, a landslide inventory map is a landslide database that records the location, the date of occurrence (if known), and the landslide type, parameters, and properties (Malamud et al., 2004, Guzetti et al., 2012). A landslide inventory map is a "prerequisite dataset" for landslide susceptibility, and hazard and risk assessment (Guzetti et al., 2012). It can be prepared using different techniques depending on the purpose of the inventory, the extent of study area, the scale of the base maps, the scale, resolution and characteristics of available imagery, and the skills and experience of the investigators (Guzetti et al., 2012; van Westen et al., 2006).

\subsubsection{Bivariate Statistical Analysis}

The fundamental concept of bivariate statistical analysis is to determine the relationship between spatial landslide distribution and landslide controlling factors (Guzetti et al., 1999). Only the location of landslide detachment area (landslide crown) is used in the analysis (Magliudo et al., 2008; Thiery at al., 2007) (Figure 6.1). This is based on the assumption that factors controlling the occurrence of landslides in a region in the past are the same as those that will cause landslides in the future. The analysis is done basically by comparing landslide inventory maps with a series of landslide factor parameter maps, which results in a weighted class value (Equation 6.1).

The weighted class value is determined on the density of landslides in each parameter map determined by using an Information Value method (Yin and 
Yan, 1988; Jade and Sarkar, 1993; Zezere, 2003; Lin and Tung, 2003; Yalcin, 2008). This method is also known as landslide index method (Wi), in which the weighted value for a parameter class is defined as the natural logarithm of the landslide density in the class, divided by the landslide density in the entire map (van Westen, 1997). The Wi method is based on the following formula:

$W i=\ln \frac{\text { Densclass }}{\text { Densmap }}=\ln \frac{N p i x(S i) / N p i x(N i)}{\sum N p i x(S i) / \sum N p i x(N i)}$

where Wi denotes the weight given to a certain parameter class; Densclass denotes the landslide density within the parameter class; Densmap denotes the landslide density within the entire map; Npix $(\mathrm{Si})$ denotes the number of pixels which contain landslides, in a certain parameter class and Npix ( Ni) denotes the total number of pixels in a certain parameter class. The natural logarithm is used to determine the influence of a certain parameter class in landslide development within the entire map. Negative values of $W i$ indicate that the presence of that particular parameter class has less effect to the landslide development, whereas positive values indicates a relevant relationship between the presence of such parameter class with landslide development (Yin and Yan, 1988; Zezere, 2002).

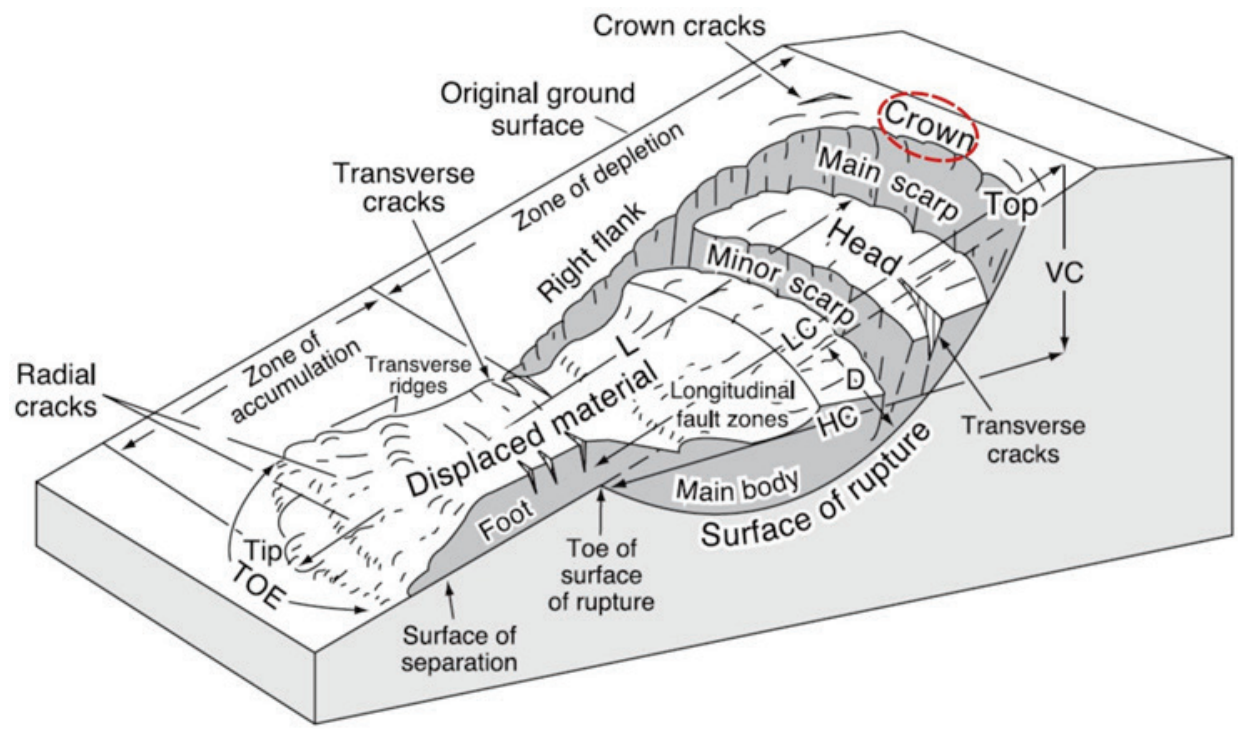

Figure 6.1 Ideal block diagram of landslide. The GPS reading for the location of landslide is taken at the landslide crown area (after Varnes 1978).

The landslide density per parameter class in each thematic map is calculated from the map crossing result between landslide inventory map and each of the parameter maps in GIS environment such as ILWIS. The Wi value of each parameter class is calculated by using the Equation 6.1. Finally, the Wi 
value of each parameter class is added together according to Equation $\mathbf{6 . 2}$ to produce a landslide susceptibility index (LSI) map or simply landslide susceptibility map.

$L S I=W i S l+W i A s+W i E l+W i L i+W i L u+W i D r+W i P r+W i D s+W i E x$

where WiSl,WiAs,WiEl, WiLi,WiLu,WiDr,WiPr, WiDs and WiEx are weighted values for slope, aspect, elevation, lithology, land use, distance to road, proximity to river, distance to structural geology and road excavation time, respectively. The landslide susceptibility index $(L S I)$ is then reclassified into five classes (very low, low, moderate, high and very high) of landslide susceptibility zones map. The "predictive power" of the map is assessed by analysing their success rate (Chung and Fabbri, 1999). In this research, the prediction rate could not be produced because multi temporal landslide data were not available. Nevertheless, the success rate result itself could indicate the quality of the map; i.e. how good the resulting weight scores describing the landslide pattern in this area (van Westen et al., 2003).

\subsubsection{Validation of LSM and assessment of landslide causal parameters effects}

Landslide susceptibility maps without any validation are worthless and may not have any scientific significance (Chung and Fabbri, 2003). Validation is essential in order to ascertain that the model can be applicable for practical purposes. Several methods to validate the quality of susceptibility map are available in the literature (such as Chung and Fabbri, 2003; Guzetti et al., 2006; Frattini et al., 2010).

In this study the validation is based on the "success rate curve" (van Westen et al., 2003), which explains how well the model and controlling factors predict landslides. Success rate is calculated by ordering the landslide susceptibility index value of all pixels in the map in descending order, and then categorize it into 100 classes with $1 \%$ cumulative intervals. The cumulative percentage of observed landslides is plotted against the cumulative percentage area of the susceptibility map. Apart from the success rate, the spatial efficiency or global statistic accuracy of the susceptibility map can also be determined qualitatively by the area under the success rate curve value (AUC) (Pourghasemi et al., 2012; von Routte et al., 2011). AUC value lies between 0 and 1 and higher $A U C$ value indicates higher prediction capability maps (Tay et al., 2014).

The relative importance of landslide causal parameter groups in the development of a landslide susceptibility map can be determined by changes in the AUC value, if one particular parameter group is excluded in the calculation of the landslide susceptibility index (Equation 6.2). If the 
exclusion of a selected parameter group results in a relatively lower AUC value, it indicates that the group is a relatively important causative factor (Tay et al., 2014; Lee and Talib, 2005). On the other hand, if the AUC value increases, that particular group may not have any significant effect on the occurrence of landslides. Therefore, this particular group should be excluded in the production of the final LSM.

\subsection{Data Preparation}

\subsubsection{Landslide Inventory Map}

A landslide inventory map for the northern Kota Kinabalu area is made based on; (1) landslides interpreted on three series of aerial photographs flown during the period of 1969 to 1972 at scale 1:30,000 and on Landsat TM satellite images with 30 metre resolution; (2) landslides reported in investigations done by the Minerals and Geoscience Department of Malaysia (e.g. Tating, 2003; Alvin, 2011; Rodeano et al., 2011a), and (3) landslides mapped during the fieldwork campaign in this research. Landslide inventory data are plotted on a base map with a scale of $1: 25,000$ (Figure 6.2). Due to the differences in database inventory format, only the landslide location and types are used in this research.

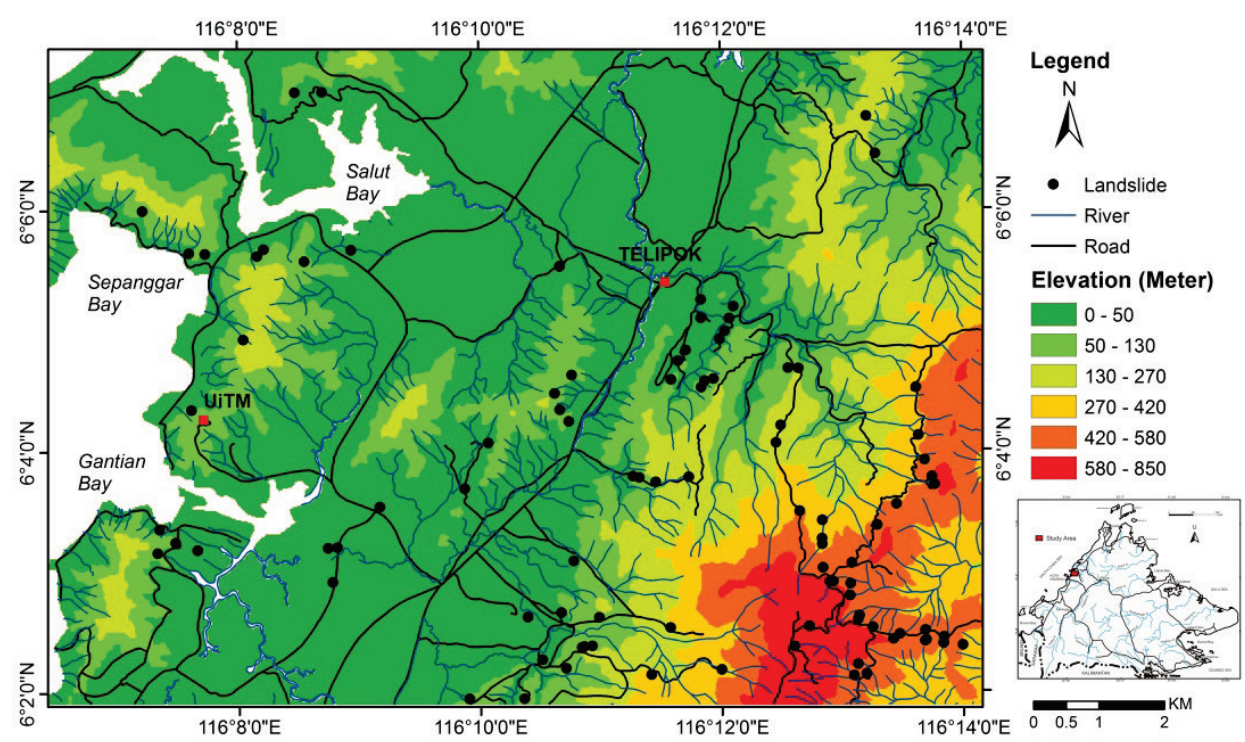

Figure 6.2 Location of landslides.

A total of 105 landslides are recorded in the study area. Most of the landslides are of a shallow translational type and some are rotational. Most of the landslides occurred at the southeastern and middle part of the study area. Most of the landslides cover an area between 150 and 1,500 $\mathrm{m}^{2}$ with 
the largest being about $36,000 \mathrm{~m}^{2}$. During the data collection fieldwork, landslides have been characterised according to the WP/WLI (1990) definition. If the date of landslide occurrence was known, the information is recorded in the landslide inventory. However, the dates of occurrence of most of the landslide incidents were unknown except for some catastrophic landslides that involved the loss of life.

\subsubsection{Selection of Landslide Preparatory Parameters}

Statistical methods in determining landslide susceptibility are based on two assumptions; (1) future landslides are likely to occur in the same area where a landslide has occurred before; (2) areas with similar geo-environmental conditions will likely have similar landslides (Guzzetti et al., 1999; Fell et al., 2008). If correct these assumptions imply that the probability of the spatial location of landslides in the future is similar to the historical landslide distribution and the preparatory parameters for landslide initiation are similar. Generally, the selection of preparatory factors depends on the nature of the study area, landslide type, failure mechanism, the scale of analysis and a priori knowledge of the main causes of landslides (Guzzetti et al., 1999; Glade and Crozier, 2005; Jaiswal et al., 2010). The selected parameters should also be operational, measurable, well representing the whole study area and vary spatially (Yalcin, 2008).

In this research there are eleven preparatory parameters used for the susceptibility analysis based on field observation, landslide characteristics, and information from previous studies and reports. These are the geological parameters (lithology, structural geology, and structural geology density) topographical parameters (slope angle, slope aspect, and elevation), anthropogenic factors (land cover/land use, distance to road, and road construction duration), and hydrological parameters (distance to river and drainage density).

\subsubsection{Geological parameters}

Geological parameters such as lithology and tectonic structure are important parameters in landslide susceptibility mapping. This is because lithological and structural variations may often lead to different susceptibility to geomorphological processes, resistance against weathering and variation in geotechnical properties (Das et al., 2010; Lee and Taib, 2005; Dahal et al., 2008; Dai et al., 2001, Ayalew and Yamagishi, 2005). The geology of the area consists of thick sequences of fine- to medium-grained sandstone and red and/or gray shale beds belonging to the "Crocker Formation" (Chapter 2). The sedimentary sequence is divided into two main lithological units i.e. Sandy Sequence and Shaly Sequence based on the lithological dominance (Tating et al., 2013) (Figure 6.3). 
(a)

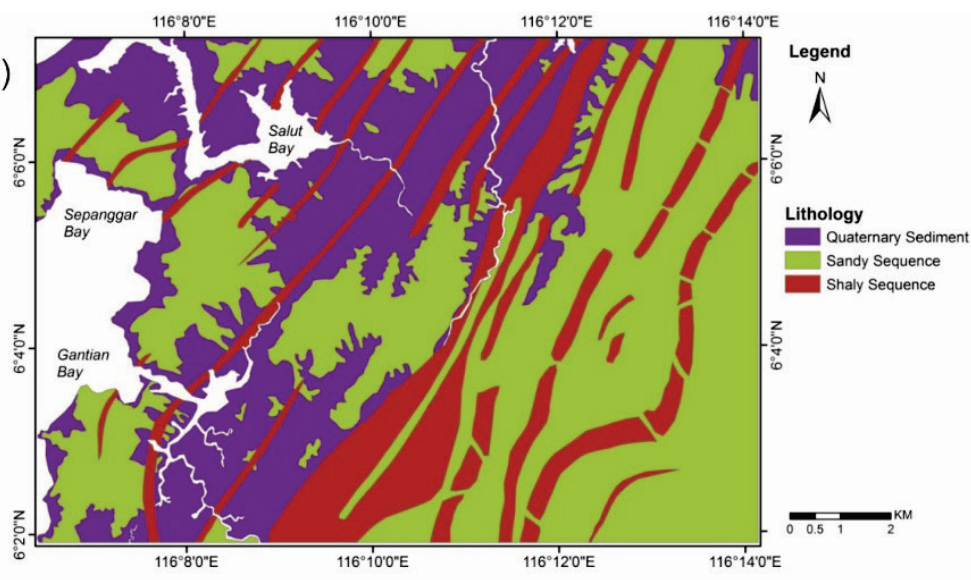

(b)

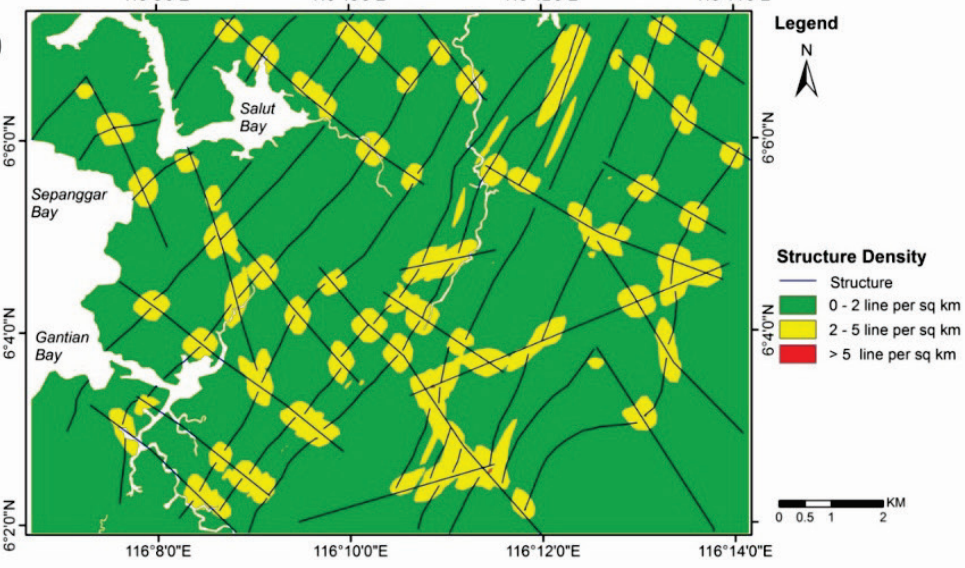

(c)

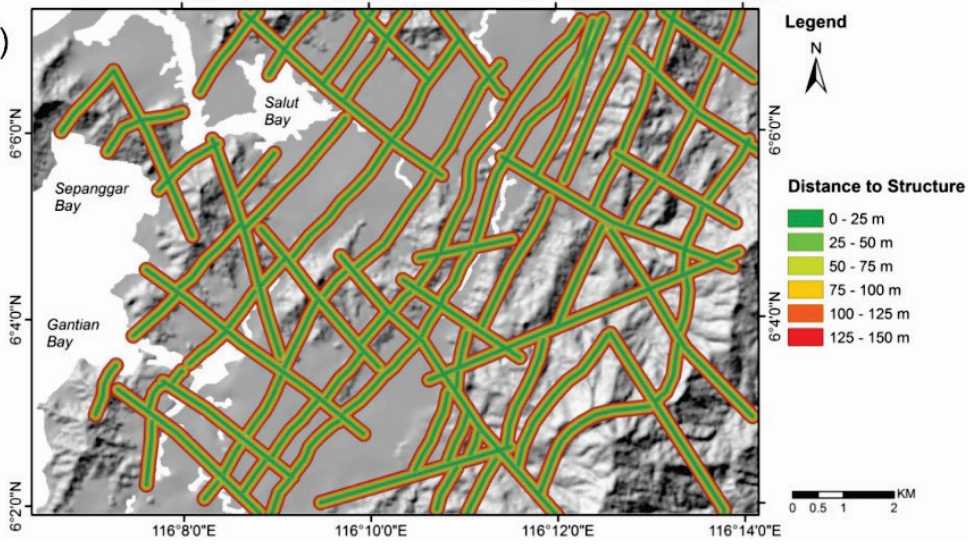

Figure 6.3 Geological factor maps used for the landslide susceptibility assessment; a. lithology; b. distance to structural geology; c. structural geology density.

The Sandy sequence consists predominantly of sandstone, which is more competent and more resistant to geomorphological processes compared to 
the Shaly sequence. Recent unconsolidated sediment (Quaternary Sediment in Figure 6.3a) occupies most of the coastal plain and valleys between the hills. It consists of coastal and fluvial deposits.

The items in the parameter tectonic structures are mainly faults either thrust or strike-slip faults. Thrust faults are normally associated with highly fractured zones. The rock mass consists of rock fragments with varying size embedded in a shaly and sandy matrix. This zone may contribute to slope instability and foundation problems. Weathering in fractured and sheared rock zones may be very deep and the rock mass strength is lower (due to high number of discontinuities) than in surrounding areas. The influence of geological structures is related to the proximity to the structural features and to the density of the structures. About 50 lineaments have been mapped from aerial photographs and satellite images by visual interpretation and verified in the field. These lineaments were buffered with $25,50,75,100$, 125 and $150 \mathrm{~m}$ distance from the lineaments mid-line (Figure 6.3b) to determine the effect of proximity to the structural geology features. The structural density, which is defined as the number of line elements of fixed length in a fixed area (Suzen and Doyuran, 2004) is classified into 3 classes (Figure 6.3c).

\subsubsection{Topographical Parameters}

Topographical parameters consist of elevation of hill, slope aspect, and slope angle. Slope aspect and slope angle are frequently used parameters in the landslide susceptibility analysis. According to Dai and Lee (2002), elevation may influence the susceptibility to landslides due to the differences in the characteristics of slope material at a certain elevation of hill. However, this is may be site specific and may not be true in the study area. In the study area, the magnitude and degree of disturbance due to construction of infrastructure especially the road network, on the side of a slope at a certain elevation of a hill is more applicable. Therefore, for the northern Kota Kinabalu area, elevation is included in the anthropogenic factors during the LSM analysis. Slope cuts are more extensive on the side of a slope compared to slope cuts at the hill ridge or slope toe. The elevation range in the study area is from 0 to 850 metres a.m.s.l. and was reclassified into 17 classes with an interval of 50 metres (Figure 6.4a).

Slope aspect, which is the direction of the maximum slope dip of the terrain surface, also influences the occurrence of landslides. Aspect-related parameters such as exposure to sunlight, drying winds, and rainfall during the southwestern monsoon may influence the moisture content and vegetation, thus may affect the moisture content, soil strength, erosion potential, and susceptibility to landslides. The slope aspect was also derived 
from the Digital Terrain Model (DTM) generated from topographic maps using Arc GIS 10 and reclassified into 9 classes with the interval of $45^{\circ}$ (Figure 6.4b).

(a)

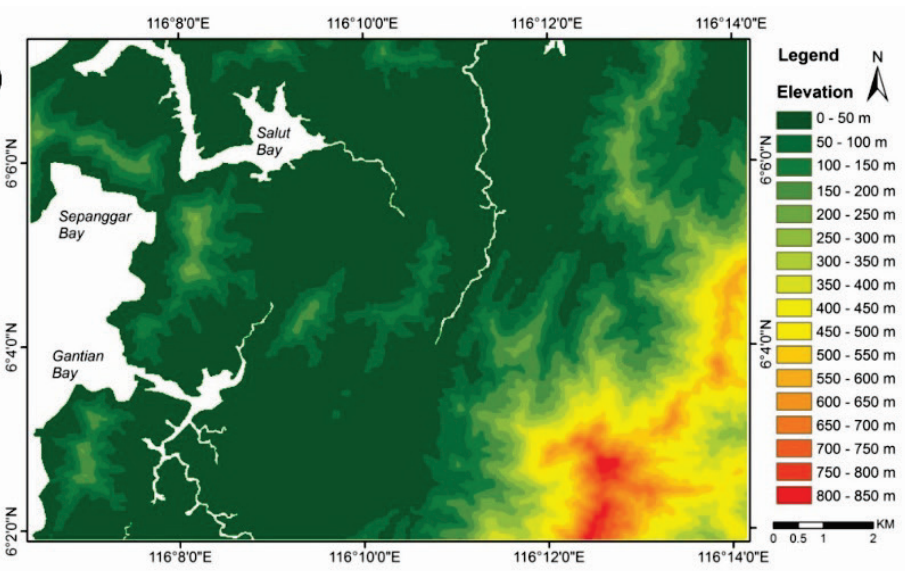

(b)

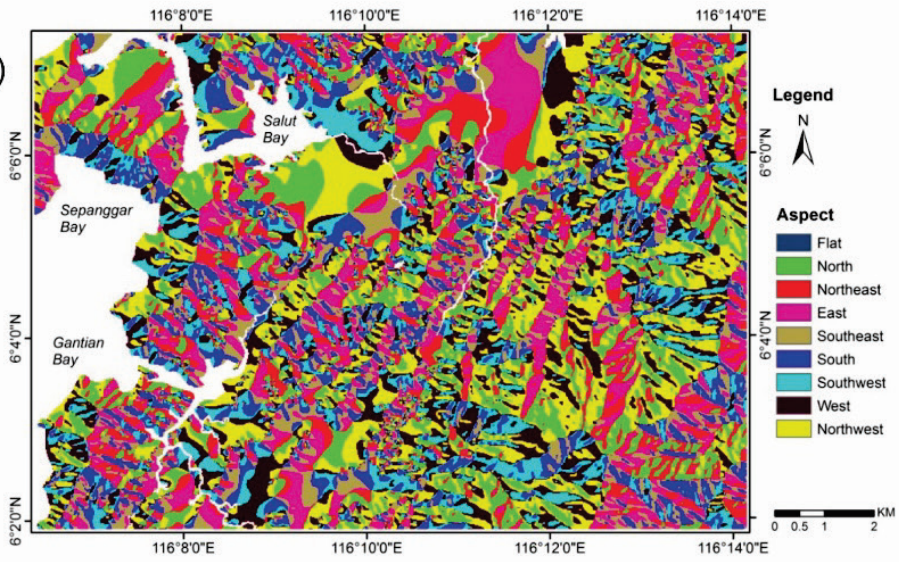

(c)

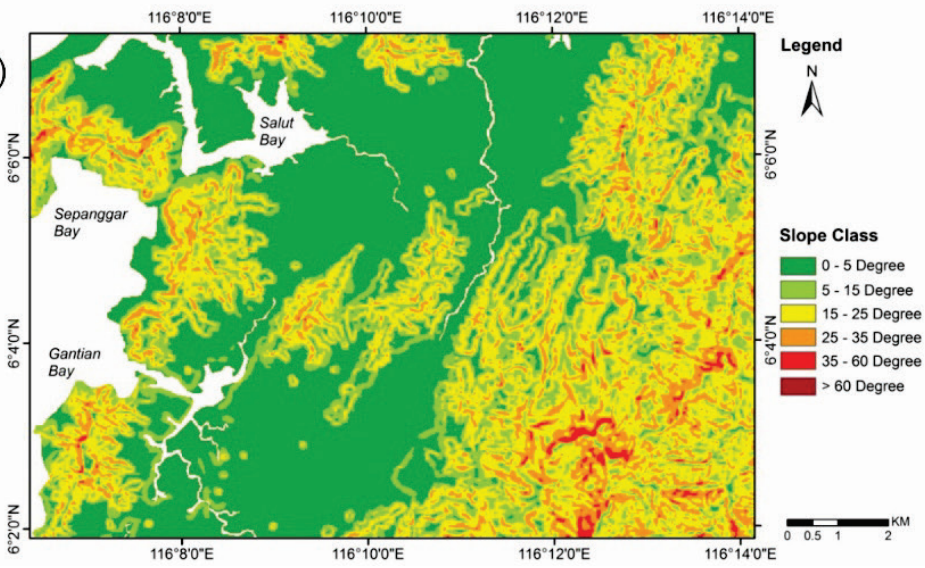

Figure 6.4 Topographical factor maps used for the landslide susceptibility assessment; a. elevation; b. aspect; c. slope angle. 
The slope angle has a very large influence on the susceptibility to landslides. The influence of slope angle is related to the shear strength of the material of the slope. Generally, landslide frequency will increase with higher slope angle until maximum frequency is achieved, subsequently followed by a decrease of frequency (Dai and Lee, 2002). Slope angle was derived from a digitized topographic map with 20 metres contour spacing that was interpolated in a $20 \mathrm{~m}$ regular DTM using Arc GIS 10. The slope map was reclassified into 6 classes (Figure 6.4c).

\subsubsection{Anthropogenic Factors}

Anthropogenic factors are related to the effect on the environment resulting from human activities. It includes infrastructure construction and land use/land cover. Construction of roads has significantly increased the frequency of slope failure along the road corridor. Slope excavation will disturb the strength - stress equilibrium of slope, leading to instability and it may result in development of tension cracks that facilitate the infiltration of water into the slope material. It may also increase the rate of slope material and mass deterioration. Excavation may also expose the barren slope material and mass to weathering agents thus further enhancing the rate of deterioration. Man-made fills without vegetation are also prone to erosion and weathering increasing their susceptibility to failure. However, landslides may only be confined to a certain distance from the road line. In order to investigate the effective range of road construction activities to landslide susceptibility, it was buffered with 25, 50, 75, 100, 125 and 150 m distance from the road line (Figure 6.5a).

Another important causal factor for landslides is the road excavation time or cut slope exposure time, which is related to the deterioration of rock mass with time. Even though man-made cut slopes have been designed to be stable for a certain period of time, the stability will decrease in the course of time. Some cut slopes may fail soon after the construction, due to stress relief and weathering (Tating et al., 2013; Huisman et al., 2006). Roads in the study area have been constructed from about 1 to 35 years ago. Therefore, road excavation time was classified into two classes, i.e. road construction less than 30 years and more than 30 years (Figure $\mathbf{6 . 5 b}$ ).

Land use changes especially conversion of virgin forest into plantations, shifting cultivation, and infrastructure construction activities may result in an area more susceptible to landslides. These activities may also result in barren slopes exposed to erosion that may eventually lead to landslides. Regeneration of secondary forest may have reduced root strength compared to the original vegetation, thus increasing the landslide potential (Razak, 2014; Sidle and Ochiai, 2006; Sidle et al., 2006). Land use/ land cover factor 
is prepared from digital topographic map of 1: 25000 and satellite imagery coupled with an extensive field survey. Land use factor is classified into 7 classes (Figure 6.4c).

(a)

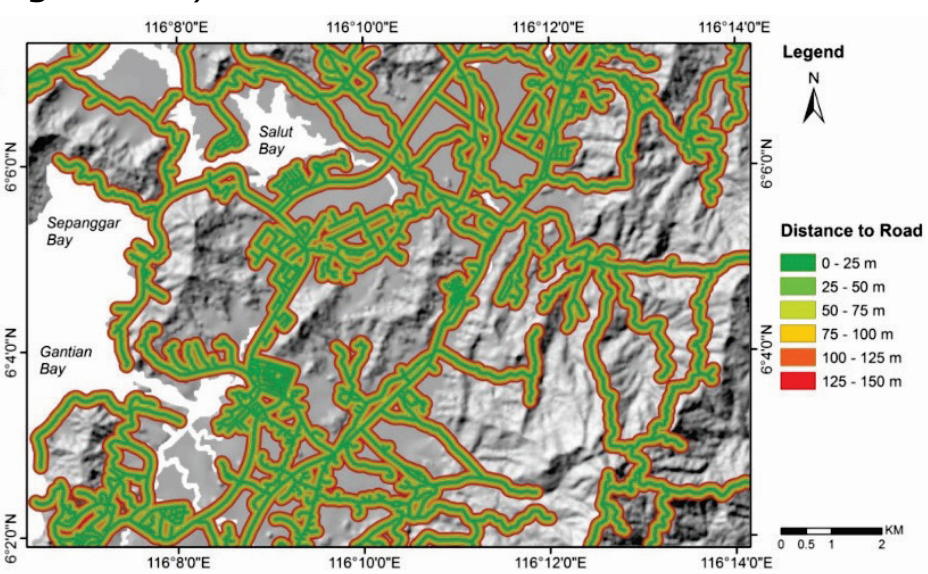

(b)

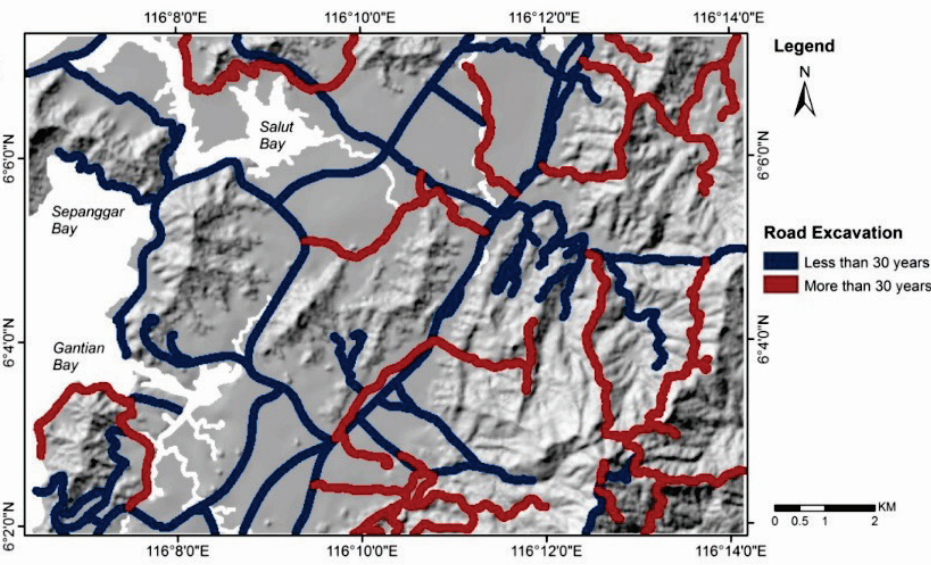

(c)

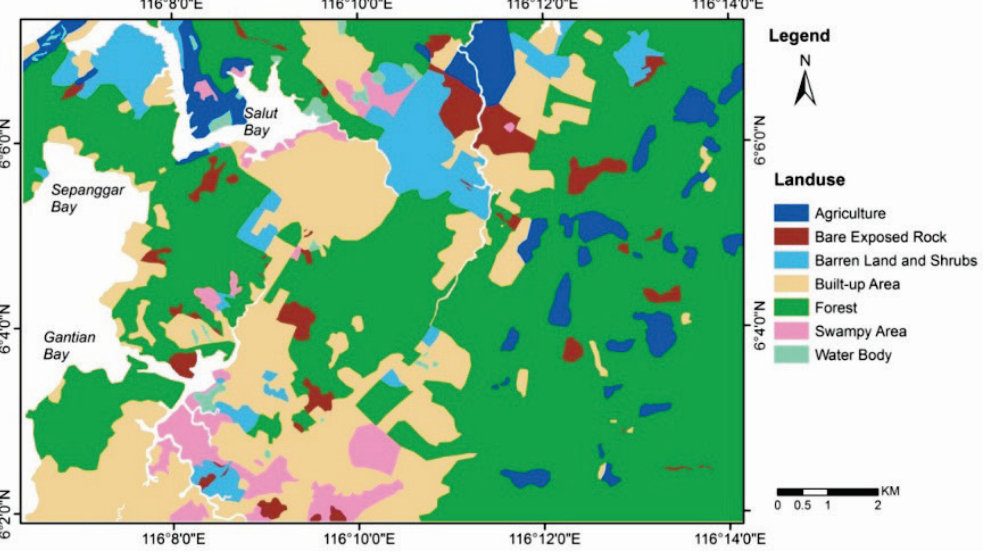

Figure 6.5 Anthropogenic factor maps used for the landslide susceptibility assessment; a. distance to road; b. road excavation time; c. landuse/landcover. 


\subsubsection{Hydrological Parameters}

Hydrological factors refer to the proximity of slope areas to river systems and drainage density. Drainage density is the ratio of total drainage length per square kilometre area and calculated using line density tools in Arc GIS 10. The proximity to the drainage system may adversely affect slope stability by eroding the slope toe or/and saturating the toe material, whereas the density contributes to the regional hydrogeological properties of an area such as groundwater content. Six different buffer zones were delineated along drainage system to determine the effect of proximity to the drainage on occurrence of landslide. The buffer zones are 25, 50, 75, 100, 125 and 150m from the drainage centre line, whereas the density is classified into 4 classes (Figure 6.6).

(a)

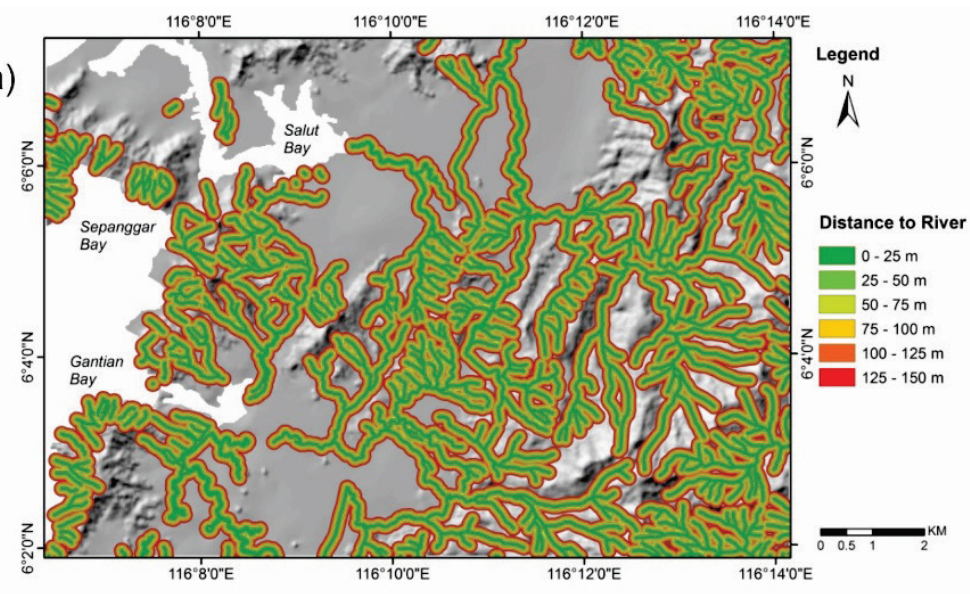

(b)

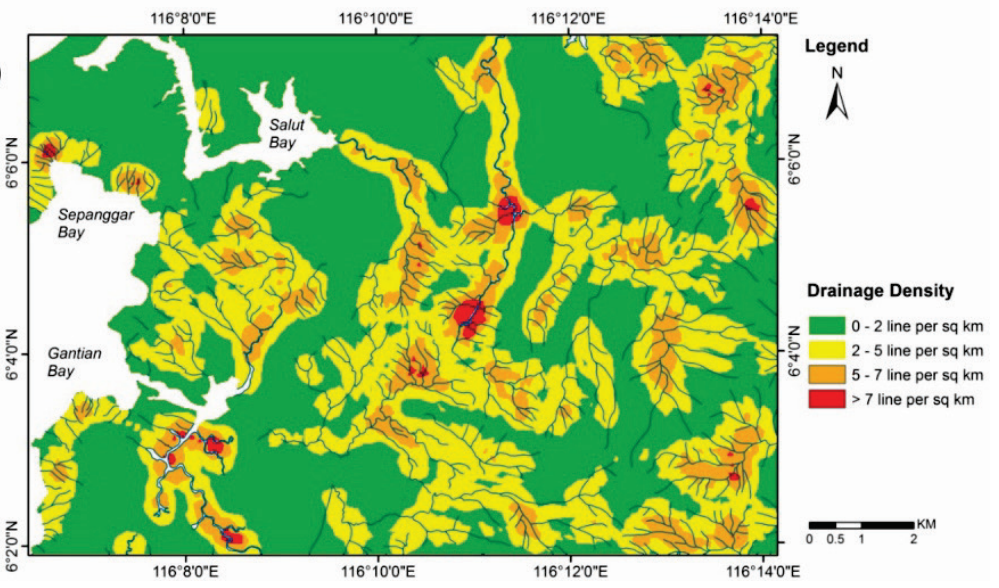

Figure 6.6 Hydrological factor maps used for the landslide susceptibility assessment; a. distance to river; b. drainage density. 


\subsection{Result}

\subsubsection{Landslide parameter/factor class weight}

The relationship between landslide distribution and landslide controlling factors is shown in Table 6.1. The relationship is reflected by the weight value of parameter class. Negative weight value of any parameters class shows that their presence may not contribute to the occurrence of landslides. Positive weight value indicates that the particular parameter class characteristics or occurrence may enhance the probability of landslides. A weight value around zero $( \pm 0.1)$ contributes to the neither presence nor absence of landslides.

Table 6.1 Weight values obtained from the bivariate statistical method for all parameter classes.

\begin{tabular}{|c|c|c|c|c|c|c|c|}
\hline \multirow[t]{2}{*}{ Group } & \multirow[t]{2}{*}{ Factor } & \multirow[t]{2}{*}{ Class } & \multicolumn{2}{|c|}{ Landslide Area } & \multicolumn{2}{|c|}{$\begin{array}{l}\text { Total Area in the } \\
\text { Class }\end{array}$} & \multirow[t]{2}{*}{ Weight } \\
\hline & & & Pixel & $\%$ & Pixel & $\%$ & \\
\hline \multirow[t]{12}{*}{ Geological } & Lithology & Quaternary Sediment & 75 & 17.56 & 96978 & 30.32 & 0.0000 \\
\hline & & Shaly Sequence & 79 & 18.50 & 62637 & 19.58 & -0.4855 \\
\hline & & Sandy Sequence & 273 & 63.93 & 160272 & 50.10 & 0.2683 \\
\hline & Distance to & $0-25 m$ & 19 & 9.41 & 22357 & 9.41 & -0.6931 \\
\hline & Structure & $25-50 m$ & 27 & 13.37 & 22680 & 13.37 & -0.2877 \\
\hline & & $50-75 m$ & 42 & 20.79 & 22143 & 20.79 & 0.1719 \\
\hline & & $75-100 m$ & 35 & 17.33 & 21174 & 17.33 & 0.0606 \\
\hline & & $100-125 m$ & 39 & 19.31 & 20274 & 19.31 & 0.1719 \\
\hline & & $125-150 m$ & 40 & 19.80 & 19397 & 19.80 & 0.2719 \\
\hline & Structure & $0-2.5 \# / \mathrm{km}^{2}$ & 379 & 88.76 & 292482 & 85.07 & 0.0800 \\
\hline & Density & $2.5-5 \# / \mathrm{km}^{2}$ & 48 & 11.24 & 51308 & 14.92 & -0.2877 \\
\hline & & $5-7.5 \# / \mathrm{km}^{2}$ & 0 & 0 & 21 & 0.01 & -2.4849 \\
\hline \multirow[t]{14}{*}{ Topogra-phical } & Aspect & North & 127 & 29.74 & 42865 & 13.34 & 1.6363 \\
\hline & & Northeast & 31 & 7.26 & 34734 & 10.81 & -0.3677 \\
\hline & & East & 36 & 8.43 & 43146 & 13.43 & -0.4855 \\
\hline & & Southeast & 25 & 5.85 & 34099 & 10.61 & -0.6190 \\
\hline & & South & 24 & 5.62 & 34349 & 10.69 & -0.6190 \\
\hline & & Southwest & 43 & 10.07 & 34865 & 10.85 & -0.0800 \\
\hline & & West & 19 & 4.45 & 46231 & 14.39 & -1.1787 \\
\hline & & Northwest & 122 & 28.57 & 50997 & 15.87 & 0.6131 \\
\hline & Slope & 0 - 5 Degree & 47 & 11.01 & 137499 & 42.78 & -1.4663 \\
\hline & & $5-15$ Degree & 112 & 26.23 & 60976 & 18.97 & 0.3254 \\
\hline & & $15-25$ Degree & 168 & 39.34 & 86405 & 26.88 & 0.3795 \\
\hline & & 25 - 35 Degree & 95 & 22.25 & 34103 & 10.61 & 0.7673 \\
\hline & & 35 - 60 Degree & 5 & 1.17 & 2461 & 0.77 & 0.4308 \\
\hline & & > 60 Degree & 0 & 0.00 & 0 & 0.00 & -2.5649 \\
\hline \multirow[t]{17}{*}{ Anthropogenic } & Elevation & $0-50 m$ & 196 & 45.90 & 178454 & 55.51 & -0.1671 \\
\hline & & $50-100 m$ & 93 & 21.78 & 41300 & 12.85 & 0.5705 \\
\hline & & $100-150 m$ & 14 & 3.28 & 25141 & 7.82 & -0.7732 \\
\hline & & $150-200 m$ & 2 & 0.47 & 17596 & 5.47 & -2.5649 \\
\hline & & $200-250 m$ & 18 & 4.22 & 9892 & 3.08 & 0.3254 \\
\hline & & $250-300 m$ & 8 & 1.87 & 5792 & 1.80 & 0.0741 \\
\hline & & $300-350 m$ & 2 & 0.47 & 6318 & 1.97 & -1.4663 \\
\hline & & $350-400 m$ & 15 & 3.51 & 6714 & 2.09 & 0.5261 \\
\hline & & $400-450 m$ & 13 & 3.04 & 7003 & 2.18 & 0.3795 \\
\hline & & $450-500 m$ & 22 & 5.15 & 6746 & 2.10 & 0.9316 \\
\hline & & $500-550 m$ & 23 & 5.39 & 6112 & 1.90 & 1.0726 \\
\hline & & $550-600 m$ & 14 & 3.28 & 3791 & 1.18 & 1.0460 \\
\hline & & $600-650 m$ & 3 & 0.70 & 2399 & 0.75 & 0.0000 \\
\hline & & $650-700 m$ & 0 & 0.00 & 1797 & 0.56 & -2.5649 \\
\hline & & $700-750 m$ & 4 & 0.94 & 1276 & 0.40 & 0.8690 \\
\hline & & $750-800 m$ & 0 & 0.00 & 825 & 0.26 & -2.5649 \\
\hline & & $800-850 m$ & 0 & 0.00 & 309 & 0.10 & -2.5649 \\
\hline
\end{tabular}




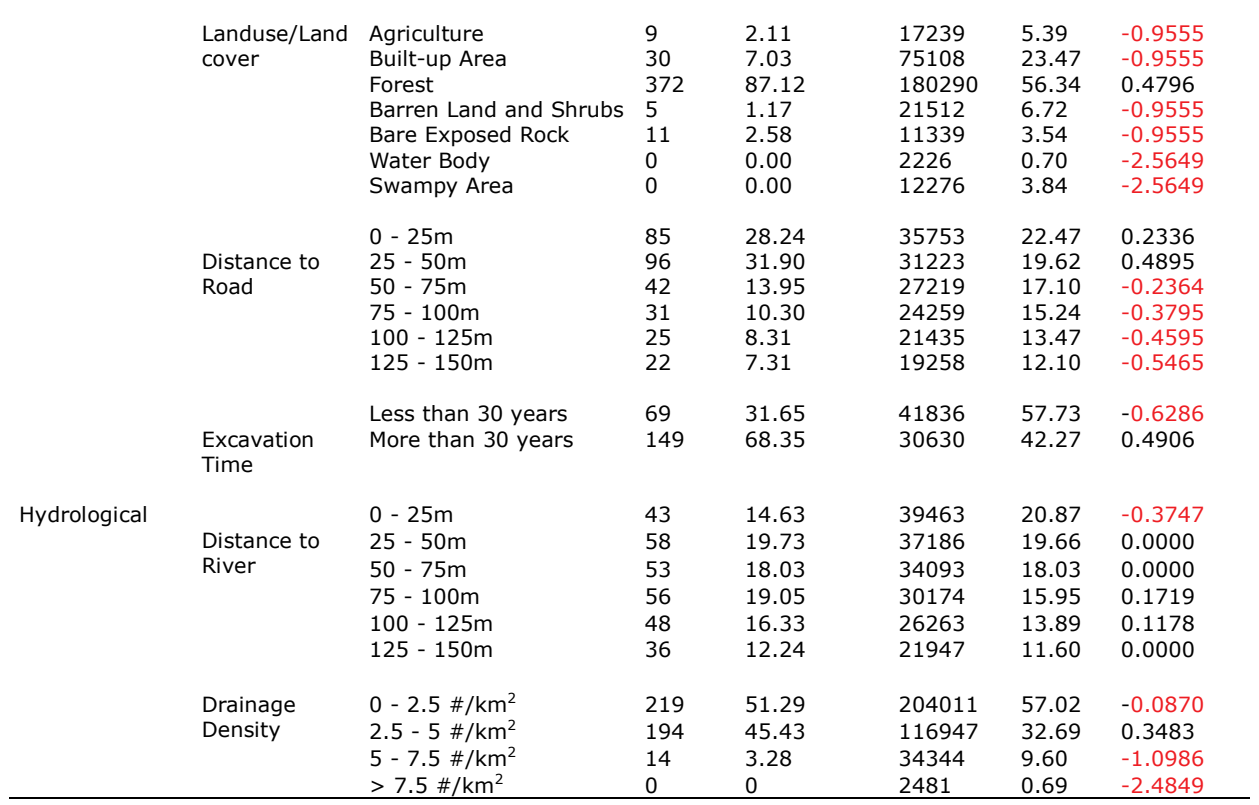

\subsubsection{Assessment of landslide causal parameters effects and validation of $L S M$}

The relative contribution of each group can be determined by the AUC value of "success rate curve" if one group was excluded in the LSI analysis (see Section 6.3.3). The percentage of the AUC indicates the relative accuracy of the landslide susceptibility map (LSM). Based on Table 6.2 and Figure 6.7, it shows that anthropogenic and topographical groups have a significant effect on the relative accuracy of the landslide susceptibility map (LSM). By excluding either anthropogenic or topographical groups, the AUC will reduce to about $5 \%$; i.e. from $80.22 \%$ to about $76 \%$. Whereas, by excluding the hydrological group, it only decreases less than $1 \%$ of the AUC to about $79.5 \%$. However, the exclusion of geological group increases the AUC value about $1 \%(80.2 \%$ to $81.1 \%)$, which indicates no significant impact on the accuracy of the LSM. 
Table 6.2 AUC values obtained from different analysis scenario of landslide susceptibility index (LSI).

\begin{tabular}{|c|c|c|c|c|}
\hline Scenario & $\begin{array}{l}\text { Parameter groups } \\
\text { excluded }\end{array}$ & $\begin{array}{l}\text { Area under } \\
\text { curve (AUC) }\end{array}$ & $\begin{array}{l}\text { *AUC value } \\
\text { differences } \\
(\%)\end{array}$ & $\begin{array}{l}\text { Landslide } \\
\text { susceptibility map } \\
\text { accuracy }(\%)\end{array}$ \\
\hline 1 & None & 0.8022 & - & 80.22 \\
\hline 2 & $\begin{array}{l}\text { Anthropogenic } \\
\text { Parameters }\end{array}$ & 0.7617 & 4.05 & 76.17 \\
\hline 3 & $\begin{array}{l}\text { Topographical } \\
\text { Parameters }\end{array}$ & 0.7630 & 3.92 & 76.30 \\
\hline 4 & Hydrological Parameters & 0.7947 & 0.75 & 79.47 \\
\hline 5 & Geological Parameters & 0.8108 & -0.86 & 81.08 \\
\hline 6 & $\begin{array}{l}\text { Hydrological and } \\
\text { Geological Parameters }\end{array}$ & 0.8038 & -0.16 & 80.38 \\
\hline
\end{tabular}

Note: *AUC differences = Scenario 1 (all parameter included)- Successive Scenarios (specified parameter group excluded)

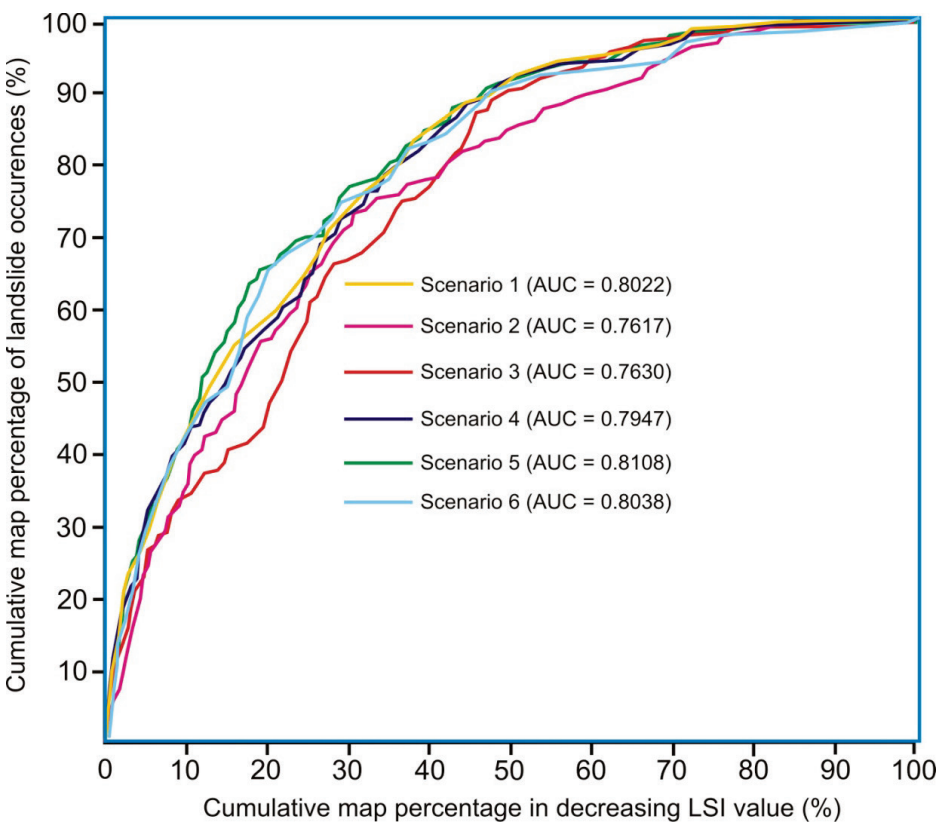

Figure 6.7 The comparison between "success rate curve" by using all parameter group and without a particular parameter group. The areas under the curve (AUC) for the different scenario are shown in the parenthesis.

\subsubsection{Landslide Susceptibility Map}

The final LSM is produced by using the LSI with the highest AUC value (i.e. without the geological parameters). The calculated landslide statistical index (LSI) value ranges from about -8.408 to 4.662 . The index value is reclassified into five susceptibility classes (very low, low, moderate, high, and very high) by using natural break classification. The final susceptibility map is shown in Figure 6.8. The percentages of area classified as very low, low, moderately, high, and very high susceptibility are 16.94, 26.91, 20.74, 22.54 , and $12.88 \%$, respectively. 


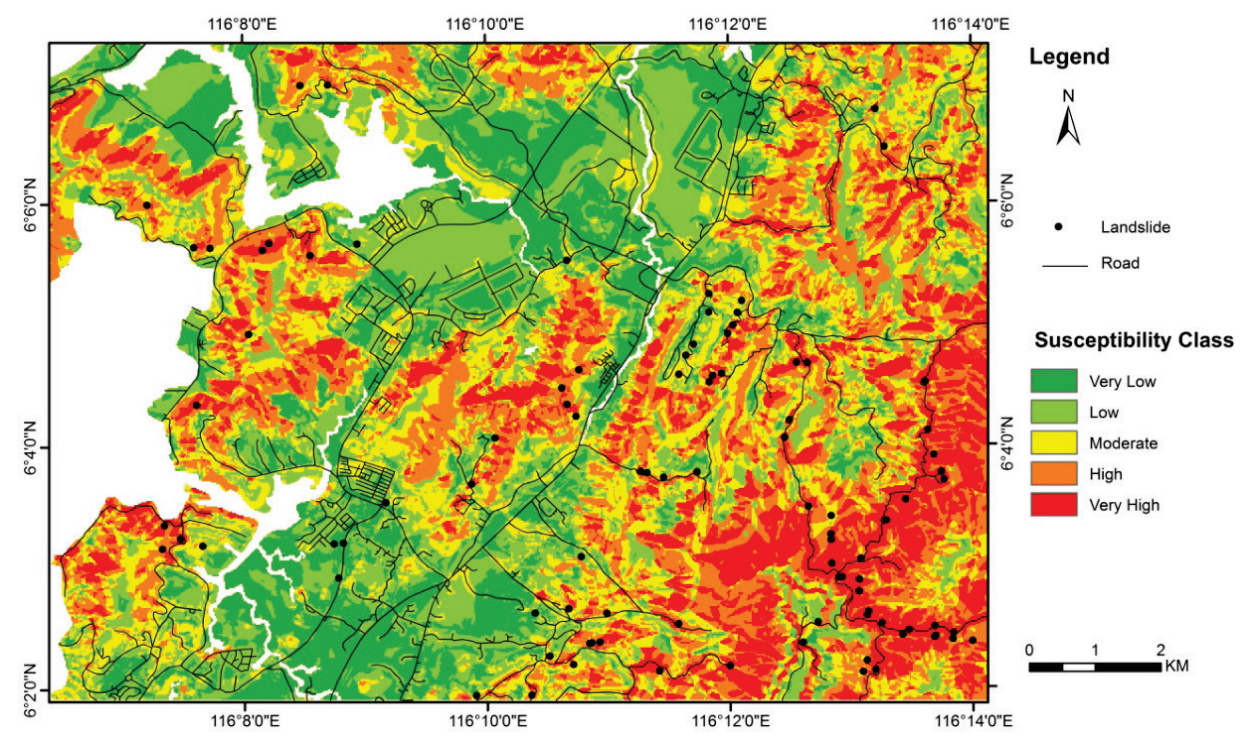

Figure 6.8 Final susceptibility map obtained by using three landslide causal parameter groups, topographic, anthropogenic, and hydrological factors. The landslide inventory location is also shown in the map.

\subsubsection{Validation of Landslide susceptibility map}

The "success rate curve" for the susceptibility map is shown in Figure 6.9. Based on the success rate graph, it shows that the first $30 \%$ of the classes with the highest value in the susceptibility map can predict about $77 \%$ of all landslides in the area. The AUC value for the final LSM is 0.8108 , which can be considered as excellent in discriminating area with landslide occurrence (Hosmer and Lameshow, 2000) and indicates overall success rate is about $81.08 \%$. 


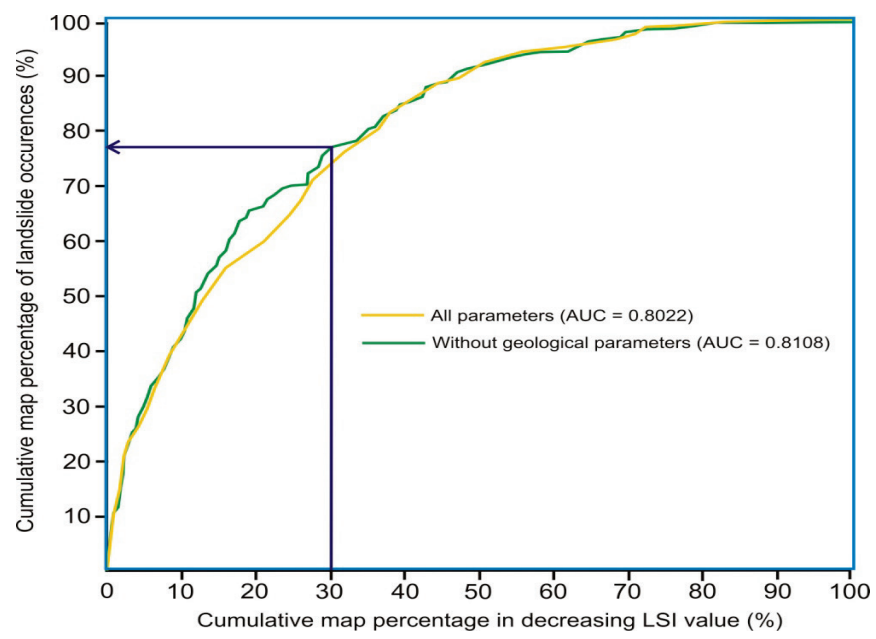

Figure 6.9 The "success rate curve" of the final landslide susceptibility map (green line).

\subsection{Discussion}

Even though geological parameters are considered among the important parameters in landslide susceptibility assessment, the inclusion of these parameters in the analysis reduce the accuracy of the LSM of the study area. This may indicate that geological parameters have a very small effect on landslide occurrence in the study area. The reasons may be due to the low variability in rock formation and simplification of the lithological units. The entire study area is underlain by only one rock formation i.e. Crocker Formation which comprises of three main geological units (see chapter 2). However, the lithological units could not be mapped at the current map scales, thus the geological units have been reclassified into two main groups i.e. Sandy and Shaly sequences.

The weight analysis result shows that the sandy sequence has a positive weight value suggesting that it is prone to landslides. On the other hand, the Shaly sequence which occupies most of the low-lying area and between the sandstones beds shows a negative weight value suggesting that it is less prone to landslides. Even though, it seems that the sandy sequence is more susceptible to landslides, in fact due to the intercalation of these units, any disturbance on either one of the units may result in landslides. The weight value of quaternary sediment class suggests that it contributes to neither the presence nor absence of landslides. Most of the quaternary sediment occupies the river valleys and coastal area.

A similar weight result is shown by the proximity to geological structural features. Negative weight values are observed for the distance from 0 to 50 
metres which indicate the absence of landslides nearer to the structural features. This is also contrary to what could be expected as the disturbance by tectonic activities mostly contributes to the occurrence of landslides. Structure density parameter classes also show a similar result; a higher density of geological structures does not influence the occurrences of landslides. Landslides may not occur at areas adjacent to geological structures unless it is excavated and exposed to the deteriorating elements. The occurrences of landslide low-density area may be attributed to other factors such as land use activities.

The significant landslide causal groups are the topographical parameters. Based on the analysis result (Table 6.1), the north and northwest direction slope aspect are more susceptible to landslide as indicated by the positive weight values. The high occurrence of landslides on these slopes is related to the rainfall intensity distribution patterns brought by the southwestern Monsoon. Landslide occurrences are also associated with the slope class between 25 - 35 degrees. Slopes gradient less than 5 degrees and more than 60 degrees are less susceptible to landslides.

In general, anthropogenic parameters are related to the effect on the environment resulting from human activities such as construction of infrastructure and land use activities. Based on the land use/land cover factor, landslides mostly occurred within the forested areas. However, this does not mean, that land use/land cover are the main causal factors for landslides. As the forested area coverage is the largest, the probability of landslides occurrence is also high. Factors such as the presence of most north and northwest slope aspects in combination with relevant slope gradients within the forested area may be more important. Furthermore, human activities such as shifting cultivation, road construction, and deforestation for private residential areas are mainly carried out at these areas.

Most slope failures occurred within the range from 0 to 50 metres from the road routes. The landslides incidents are related to the disturbance and exposure to deterioration due to the construction of roads. The analysis results also show that landslides mostly occurred at roads constructed more than 30 years old. This is likely due to the deterioration or weathering of the cut slopes. The geotechnical parameters (intact rock strength, and rock mass cohesion and friction) of slope material and mass reduce with time and thus reducing the factor safety. Generally, roads constructed less than 30 years are less prone to landslides.

Some literature claims that the rock mass deterioration (i.e. weathering) factor is less applicable and difficult to apply in regional scale analyses (van Westen et al., 2008; Corominas et al., 2013). Usually, weathering effects are 
reported to be useful only in large and detailed scale assessment by using appropriate methods such as deterministic landslide analysis (Soeters and van Westen, 1996) and geotechnical methods such as Slope Stability Probability Classification (SSPC) (Das et al., 2010). However, in this study the effect of this factor is quite significant in controlling the occurrence of slope failures along the roads (positive weight value, see Table 6.1). This may indicate that the factor can be used at the scale as used in this study, if the excavation/construction time of roads is available. It should be realized that localized effects from the presence of discontinuities or mitigation measures influence the susceptibility classes of LSM.

In relation to the elevation, most landslides occurred at the elevation classes between 350 to 600 and 700 to 750 metres. In the study area, most of the landslide incidents are not related to the properties of material at a particular elevation as was suggested by Dai and Lee (2002), but are mainly due to the concentration of anthropogenic activities at this elevation. Some villages and private residential structures are constructed at this elevation because of the beautiful scenery overlooking the coastal area and cooler weather conditions. Several private roads are also constructed to link these villages to the main road, enhancing the slope disturbance. The slope gradient at these elevations may also be of influence. At higher elevations, most of the study area consists of virgin forest and forest reserves in which any human activities are prohibited.

The influence of the hydrological parameters group is based on distance/proximity from the parameter features and density of the parameter. Density functions attribute to the regional $\left(\mathrm{km}^{2}\right)$ influence of particular parameters, whereas the proximity functions evaluate the local situation $\left(\mathrm{m}^{2}\right)$ (Suzen and Doyuran, 2004). This group has little impact on the accuracy of LSM. The analysis shows that the proximity to the main river or stream does not show a very significant relationship. Negative weight value for the distance between 0 to 25 metres indicates that the proximity to main river/stream does not influence the landslide occurrences. This is contrary to what commonly is found in this type of analyses in which the effect of river/stream has an influence on the occurrence of landslides, i.e. due to slope undercutting and saturation effects. That this effect is not found in this study is likely because most of the main rivers are located in the lowland and flat areas where the riverbanks on both sides are covered with mangrove trees. In the hilly area, most of the riverbanks are also covered with trees that may prevent bank erosion. Another reason may be that the effect of trees thriving along the riverbanks may not be well captured in this scale.

The drainage density factor map analysis shows that higher drainage density does not contribute to landslide incidents. Higher drainage density indicates 
lower water infiltration and faster removal of surface water from the area. It will reduce the amount of infiltration, thus sustain slope material geotechnical properties (Mezughi et al., 2011; Yalcin, 2008). Contrasting to the lowdensity area, water infiltration may be high which may increase the shear stress and decrease the shear strength of slope material as well as facilitate the weathering process. This effect may cause landslide incidents; however, in tropical areas, the presence of water may encourage vegetation thus enhancing slope stability due to root action. Landslides may only happen if the area becomes deforested.

The quality of the susceptibility map depends on the quality and completeness of input parameters used in the analysis. One of the most important parameters is landslide inventory data. Landslide inventories can be prepared using various methods (Wieczoreck, 1984; Malamud et al., 2004, Guzetti et al., 2012). In the present study, landslide inventory is prepared mainly through aerial photographs and satellite images interpretation, extensive fieldwork, and from historical records. However, details on landslides in the Kota Kinabalu area are very limited. Only some large volume big scale landslides that resulted in large infrastructure damage or fatalities have been investigated and reported in detail. Furthermore, the landslide terminology and parameters recorded are sometimes inconsistent. However, in landslide susceptibility assessment, location and type of landslide are sufficient for the analysis. Therefore, only the landslides with known location and type were used in the analysis.

Other problems of recognizing and recording landslides in a tropical environment are related to the rapid disappearance of landslide diagnostics features due to rapid growth of vegetation after the failure. For older landslides, it may be impossible to identify their signature in aerial photos or satellite images due to the dense forest canopy and unfavourable weather conditions (cloudy and rainy) during acquisition of optical remote sensing data (Razak et al., 2013). Therefore, most of the landslides recorded are along the roads and slope cut areas, which are easily accessible and mostly have a low-density vegetation. This may lead to spatial bias of the landslide incidents. In order to overcome these problems, non-optical satellite image such as LIDAR should be utilized in the future. LIDAR data have been successfully utilized for landslide inventory and identification under dense tropical forest in Malaysia (Razak et al., 2013).

\subsection{Conclusion}

The analyses revealed that different combinations of factors have a different contribution to the landslide occurrences and subsequently to the accuracy of the Landslide Susceptibility Map (LSM). The most important parameters are 
the anthropogenic and topographical groups, whereas the hydrological group is only slightly affecting the accuracy of LSM. Surprisingly, the least important group is the geological parameters group. This may be related to low variability and generalization of the lithological units. The result is determined by the bivariate statistical method, it is recommended that further study use different methods such as the multivariate statistical method to determine the role of each factor.

Even though the selection of conditioning factors is based on the consideration of relevance and field observation, landslide characteristics and information from previous studies and reports, and the availability of data for the study area, some factors may not be suitable at the current scale (regional scale) of the study. For example, for the hydrological factor the effect of trees thriving along the riverbanks could not be incorporated in the analysis. Furthermore, it is also revealed that the role of the elevation factor is not necessarily governed by the properties of the materials at a certain elevation but rather by the land use activities at that particular elevation.

Generally, the LSM at the scale as made in this study can be used to determine the spatial location of landslide prone areas in the study area. It may help the local authorities to anticipate and evaluate the possible landslide related hazard prior to the implementation of any development projects especially at the early phases of regional development planning. The outcome from the research can also be used to enhance the regulations related to the development on hillsides in the National Guideline on Hillside and Highland Development (DTCP, 2009), which at present are based basically on the topographical and geological factors only. This study shows that human activities have great a influence and should be taken into consideration. As the study has been carried out on a regional scale, some of the influence from the causal factors could not be significantly revealed. Therefore, further study by using other susceptibility assessment method (such as analytical hierarchy process) at a proper scale is recommended. Other causal factors such as the rainfall information, soil types, and thickness should also be included in the analysis. 


\section{Chapter 7: Synthesis and Conclusions}

\subsection{Synthesis}

The aim of this research is to establish the relationship between deterioration due to weathering and stress relief, and exposure time for a particular rock type in tropical environments. Furthermore, the effect of weathering due to physical weathering (disintegration of rock mass) and chemical weathering (rock mass decomposition; precipitation of iron) on rock cut slopes have been evaluated. When dealing with cut slopes, the effect of stress release due to the removal of overburden on the durability of sandstone cannot be separated from the effects caused by weathering; therefore in this research weathering includes both weathering and stress relief.

The research is carried out in northern Kota Kinabalu, Sabah, Malaysia a tropical region on the island of Borneo. The geology in the area consists of rock formations of sedimentary origin and comprises of alternating shale and sandstone beds of various thicknesses (discussed in detail in Chapter 2). However, the research only focuses on the thick to very thick bedded sandstone unit (SST unit). Characterization of the thick to very thick bedded sandstone units is based on an extensive and detailed field observation coupled with simple physical testing of intact rock strength (IRS) in the field. First, the sandstone units are delineated into several geotechnical units based on bedding thickness, lithological type, bedding thickness ratio between different lithologies, and weathering grade. The weathering grade of the rock mass is determined visually following the methodology described in BS 5930 (1981) and ISO 14689-1:2003. Data characterization per geotechnical unit is done in quantified values based on Slope Stability Probability Classification (SSPC) methodology (Hack et al., 2003). Intact rock strength is estimated by using "simple means" methodology, which uses hand and geological hammer to estimate IRS following BS 5930 (1981) and BS 5930 (1999). The intact rock strength values obtained from this method are verified with intact rock strength values from laboratory intact rock strength tests published in the literature.

The findings of the research based on the four main research objectives that were formulated at the beginning of this research are summarized as follows.

\subsubsection{Objective 1: develop a quantitative factor for incorporation in the design of slopes to account for weathering}

The focus of the first objective is to determine the appropriate parameters of thick to very thick bedded sandstone unit (SST unit) that can be used to 
establish the deterioration processes - time relationship, and subsequently the development of quantitative factors for incorporation in the slope design (Chapter 3). The first part of the chapter gives an overview of slope material deterioration in slopes, discussion on the slope rock mass weathering with time, and rock/rock mass properties used for the development of quantitative factors. Data are collected from various cut slopes with different dates of excavation.

The development is based on the changes of intact rock strength value per unit time due to weathering process. In the literature, this is defined as "weathering intensity rate" and decreases non-linearly with time due to formation of a residual layer of weathered material protecting the underlying rock mass from further weathering. The intact rock strength value changes with exposure time following a logarithmic function as suggested by Colman (1981) and Huisman (2006). The relationship can be used to predict the future reduction of intact rock strength of the SST unit from the initial value (i.e. fresh rock) over the engineering lifetime of man-made slopes in a particular environment. Similarly, the prediction of future geotechnical parameters (i.e. cohesion and friction angle) can be done by utilizing an empirical equation for rock mass stability such as in the Slope Stability Probability Classification (SSPC) method. Thus, better man-made slope design for the full engineering lifetime can be done by anticipating the reduction of geotechnical parameters at the end of the engineering lifetime.

The specific findings of the research related to objective 1 are:

I The parameter used to establish the deterioration processes - time relationship is the intact rock strength (IRS). Their determination can be done using "simple means" which is easy, cheap, and reasonably reliable.

II The relationship between intact rock strength value of the SST unit with exposure time decreases non linearly with time and is best represented by a logarithmic function:

$I R S_{t}=105+34 \log (1+t)$

III IRS (in $\mathrm{MPa}$ ) is the intact rock strength of sandstone at the time $(t)$, $t$ is the time since exposure in years. The constant 105 is the initial value of the IRS in MPa of the fresh SST unit at the time of excavation (i.e. at exposure time 0 year), and 34 is the apparent reduction rate in $\mathrm{MPa} /(\log$ [year]) for the SST unit.

IV Linear relation of IRS reduction (i.e. IRS initial $I R S_{\text {at the time of observation }}$ ) and logarithmic of the exposure time can be used to predict the reduction of IRS for the SST unit with time:

$I R S_{\text {reduction }}=34 t+0.36$ 
The reduced IRS is equal to the IRS value ( $\mathrm{MPa}$ ) difference between fresh and weathered SST at time $t$, whereas $t$ is the logarithm of the exposure time.

$\mathrm{V} \quad$ The relationship between cohesion and friction angle for the SST unit, which is estimated by using the SSPC method, with exposure time also decreases non-linearly with time and is expressed by logarithmic functions:

Cohesion $_{t}=27004-6850.3 \log (1+t)$

Cohesion $_{t}$ (in $\mathrm{Pa}$ ) is the intact rock cohesion of SST at the time $t$, and $t$ is the time since exposure in years. 27004 is the initial value of the cohesion in Pa of the fresh SST unit at the time of excavation, and 6850.3 is the apparent reduction rate in $\mathrm{Pa} / \mathrm{log}$ [year].

Friction $_{t}=56.1-15 \log (1+t)$

Friction $_{t}$ (in degrees) is the intact rock friction angle of the SST unit at the time $t$, and $t$ is the time since exposure in years. 56.1 is the initial value of the cohesion in degree of the fresh SST unit at the time of excavation, and 153 is the apparent reduction rate in degree/log [year].

The relation allows prediction of intact rock strength reduction within the "engineering lifetime" of the slopes made in the SST unit and is likely also applicable for cut slopes in similar rock masses in an humid tropical environment, where similar weathering processes and stress relief are the reasons for degradation of the rock mass after excavation. The methodology used in this research to establish a weathering-time relation is likely applicable to areas in other climates as well.

\subsubsection{Objective 2: to evaluate the effect of weathering on the development of discontinuities in rock mass}

In this objective, the development of new discontinuities in rock masses of the SST unit due to deterioration is investigated. The results are presented in Chapter 4. An overview on the relationships between rock mass physical and geo-mechanical properties with weathering is included in chapter 4.

There are two main types of discontinuities that can be discerned in rock masses; i.e. mechanical and integral or incipient discontinuities. The former are fully developed planes of weakness in rock masses visible by the naked eye whereas the latter is still intact and normally invisible and does not form a notable mechanical weakness in the rock mass. Integral discontinuities are inherent inhomogeneities within intact rock and may develop into mechanical discontinuities as a response to changes in stress environment or weathering 
processes. In the SST unit, the development of new discontinuities is reflected by the decrease of discontinuity spacing with increasing degree of weathering. The new discontinuities consist of three dominant discontinuity sets and develop in sequential stages with advancing weathering grade. The development is analysed by using DIPs software. Persistence of discontinuities may develop not uniform over time. At higher weathering grades, newly developed discontinuity sets normally start to abut against bedding planes. Effect of weathering on discontinuity roughness is only found for the small-scale roughness, i.e. the weathering reduces the strength and number of asperities resulting in smoother surfaces with lower shear strength. An increase of discontinuity strength and roughness are anticipated if precipitation of iron oxide occurs along and on the discontinuity planes as is the case for the SST unit in higher grades of weathering (especially moderately to highly) at many locations.

The specific findings of the research related to objective 2 are:

I Three dominant sets of discontinuities developed in the SST unit due to the weathering; i.e. Discontinuity set 1 : parallel to the main bedding planes, which is oriented about NE-SW and steeply dipping either towards the SE or NW; Discontinuity set 2: conjugate joint sets oriented parallel to the bedding planes and gently dipping either towards the SE or NW; and Discontinuity set 3: joint planes striking more or less perpendicular to the bedding planes and steeply dipping towards the SW or NE.

II The spacing for all discontinuity sets decreases with increase in weathering grade due to the change of discontinuities from integral/incipient into mechanical discontinuities.

III The development of new discontinuities is sequential based on the weathering grade. In fresh SST units, only the bedding planes (discontinuity set 1 ) with some very few discontinuities from residual insitu stress are observed. Discontinuity set 2 and set 3 start to develop in slightly weathered rock. The intensity of all discontinuity sets significantly increases in higher weathering grades.

IV In the SST units, the initiation point for the formation of new discontinuities especially for discontinuity set 1 are the internal sedimentary bedding features such as parallel/cross laminations, "Bouma sequence", trace fossils, nodules, concretions, and shale laminae.

$\mathrm{V} \quad$ Changes in magnitude and orientation of the stress distribution due to rock mass excavation also contributes to the formation of new discontinuities especially for discontinuity set 2 and set 3 .

VI Weathering has little influence on the large-scale roughness of discontinuities, but has a significant effect on small-scale roughness. 
Weathering may decrease the shear strength of discontinuities due to the weathering of the discontinuity surfaces. On the other hand, it may increase the shear strength due to precipitation of strongly compacted or cemented weathering products such as iron oxide as infill materials. This is further elaborated in chapter $\mathbf{5}$.

Knowing the development due to weathering of new mechanical discontinuity sets that were not present and visible at the design stage has an important engineering implication. Slope design can be adjusted to anticipate the development of new discontinuities and new discontinuity sets over the engineering lifetime. This research shows that the effect of weathering on rock mass properties can be predicted in terms of an increase in the number of discontinuity sets as well as an increase of the number of discontinuities in each set, and the future pattern and spacing of discontinuities from studies of the integral or incipient discontinuities present.

\subsubsection{Objective 3: to assess the effect of iron solution and precipitation; and their influence on the stability of cut slopes}

In tropical areas, the effect of chemical weathering processes on rock masses is often more prevalent than the effects of physical weathering. The effect is not just confined to decomposition of rock forming minerals, but also facilitating the development of weathering zones in rock masses due to oxidation and solution processes. In this research, the effect of iron solution and oxidation processes and their influence on the general stability of cut slopes are investigated and assessed and the outcome of the research is presented in chapter $\mathbf{5}$. The source of the iron solution is from the oxidation of pyrite $\left(\mathrm{FeS}_{2}\right)$ minerals that are commonly associated with the sedimentary rocks in Kota Kinabalu area (Burgan and Ali, 2009; Hutchinson, 2005; Stephens, 1956; Van Haatum et al., 2003).

The specific findings of the research related to objective 3 are:

I Four zones have been identified in rock masses at an elevated area; i.e. "surface oxidation zone", "oxidized zone", "dissolved zone", and "slightly weathered to fresh rock zone". Iron precipitation is mostly observed in the oxidation zone either along the discontinuities or within the intact rock. Visually, the zones with oxidation are brownish to brownish-grey coloured. In the dissolved zone, the dominant process is dissolution of cementing and rock forming materials that reduces durability of the intact rock material. This zone is characterized by a greyish colour. In the slightly weathered to fresh rock zone, the effect of chemical processes is limited to staining of 


\begin{abstract}
discontinuity walls. The original colour of the intact rock is still maintained.

II Iron precipitation along discontinuity surfaces in rock masses is classified into three main types; i.e. staining, coating, and cementing. Iron concentration in each precipitation type increases from staining via coating to cementing.

III Weathering zones and its association with particular types of iron precipitation have a significant influence on the properties of discontinuities and rock masses. Iron precipitation increases the shear strength of discontinuities by stronger and harder discontinuity walls, more rough walls, and by forming bridges or continuous cemented infill. This is shown by the inter-block and sliding friction values and validated by visual observation of slope stability in the field.
\end{abstract}

The present study shows that oxidation processes will cause iron precipitation in discontinuities as well as within intact rock, while in other zones iron is removed by dissolution. The presence or absence of secondary iron minerals has a marked influence on the properties of the rock mass and discontinuities. Susceptibility to weathering of sandstone with ironprecipitated discontinuities is reduced. Therefore, in stability analyses the presences of weathering zones should probably be added to the description of the "condition of discontinuity" in rock mass descriptions and classification systems.

\title{
7.1.4 Objective 4: to assess the predictive power of several selected landslide causal parameter groups, which include the effect of deterioration processes (road excavation time) in the analysis of landslide susceptibility maps (LSM) for the northern part of Kota Kinabalu
}

The focus of objective 4 is to determine the relative importance of landslide causal parameter groups in the development of LSM. The landslide causal parameters have been group into four main group i.e. geological, topographical, anthropogenic, and hydrological groups and their relative contribution was assessed based on the area under curve (AUC) value if particular group was excluded in the analysis. Subsequently the final LSM was produced by using an appropriate combination of landslide causal parameter groups that resulted the highest AUC value.

The specific finding of the research related to objective 4 are:

I The most significant landslide causal parameter groups are the anthropogenic (elevation, land use/land cover, distance to road and 
excavation time) and topographical (slope aspect and slope angle) groups, whereas the least important are the geological (lithology, distance to structure, structure density) and hydrological (distance to river, drainage density) groups.

II The elevation factor is mainly related to the human activities rather than the properties of material at a particular elevation class. It influence may also be enhanced by other factor classes such as the slope angle.

III The role of a certain landslide causal parameter class may not be significant due to the scale (regional) of investigation.

IV The effect of rock mass deterioration factor (excavation time) is quite significant in predicting landslides. Most landslides occurred along roads constructed more than 30 years old, thus related to deterioration processes. However, landslide/slope failure, especially on cut slopes may also be governed by the discontinuities condition of rock mass and existing mitigation measures at certain parts along the roads. Thus, these areas are still subject to site investigation to verify their stability.

The LSM of the northern Kota Kinabalu area is developed based on the combination of anthropogenic, topographical, and hydrological groups with an AUC value of 0.8108 . The LSM consists of $43.85 \%$ area classified as very low to low susceptibility to landslide, $20.74 \%$ moderately susceptible and $35.42 \%$ is highly susceptible to landslide. The LSM can be used as a basic tool to anticipate and evaluate possible landslide related hazards at the early phase of regional development planning.

\subsection{Future research directions}

Generally, weathering is controlled by two main factors i.e. climatic factor and the type of parent materials (rock type). In the present study, the quantification of weathering (which also includes stress relief) is limited to the thick to very thick-bedded sandstone unit (SST unit) in a humid tropical environment only. The methodology used in this research is also likely to be applicable to areas with other climates. Therefore, it is suggested that for the future studies other types of rock in different climatic environments should be used. Further studies should also be done to other properties such as the discontinuity spacing reduction, as reduction of discontinuity spacing is controlled by weathering. The determination of intact rock strength follows the "simple means" field tests, which may be quite subjective to nonexperienced researchers. Therefore it is recommended that less experienced engineers use other testing methods such as the "Equotip hardness tester" (Verwaal and Mulder, 1993) or other relevant tools appropriate for weak weathered rock. 
This research also shows that it is possible to forecast the number of discontinuity sets and the future pattern and spacing of discontinuities in SST units from studies of the integral or incipient discontinuities present. This is likely very important for engineering works. However, this possibility needs further testing in other areas with different geological and climatic environments. It should also be noted that the assessment of the geological history of a particular area, which may influence the magnitude and orientation of stresses in rock masses, might be very useful in forecasting future discontinuity patterns and properties.

Further research is needed to determine the properties of weathering zones, which may include details on mineralogical analysis and other properties. The influence of iron precipitation on the shear strength of a discontinuity is determined based on empirical equations (Barton's discontinuity inter-block friction angle (Equation 5.2) and SSPC sliding criterion (Equation 5.3)) and it is recommended that future studies include laboratory testing for verification.

The utilization of LIDAR data for preparation of a landslide inventory map and other factor maps (slope gradient, aspect etc.) is recommended for future development of landslide susceptibility maps. LIDAR data have been used to detect landslide diagnostic features under dense tropical forest. Landslide susceptibility maps should also be prepared using a multivariate statistical method in order to determine the contribution of each landslide causative factor in the future.

It is acknowledged that different types of landslides are caused by a certain set of causal factors (van Westen et al., 2003; Zezere, 2002). Therefore, it is suggested that a better identification and selection of causal factor data input, taking into consideration landslide typology should be done in the future. In addition, a thorough knowledge and understanding of mechanical processes involved in landslides and slope failure and geological evolution of the area is essential for the personal who are going to prepare the LSM in order to correlate landslides/slope failures occurrence and onsite conditions of causal factors. 


\section{Bibliography}

Al-Agha, M.R., 2006. Weathering of building stones and its relationship to the sustainable management of the aggregate resources in Gaza Strip, Palestine. Building and Environment 41 (5), 676-686.

Alleotti, P., Chowdary, R., 1999. Landslide hazard assessment: summary review and new perspectives. Bulletin of Engineering Geology and the Environment 58 (1), $21-44$.

Alvyn, C.M. 2011. Landslide Hazard Mapping in Kota Kinabalu Area using GIS and Remote Sensing. MSc Thesis. University Malaysia Sabah. 115 pp. (Unpublished).

Amin, M.F.M., Yau, O.H., Huei, C.S., Abdullah, R.A., 2008. Characteristics of filled joint under shear loading. Bulletin of the Geological Society of Malaysia, 54, 47-51. doi: 10.7186/bgsm2008008.

Anon., 1990. Tropical Residual Soils Geological Society Engineering Group Working Party Report. Quarterly Journal of Engineering Geology and Hydrogeology 23 (1), 4 - 101. doi: 10.1144/ gsl.qjeg. 1990. 023. 001.01.

Anon., 1995. The description and classification of weathered rocks for engineering purposes. Geological Society Engineering Group Working Party Report. Quarterly Journal of Engineering Geology 28, 207 242. doi:10.1144/gsl.qjegh.1995.028.p3.02.

Applied Geotechnic Sabah, 2004. Geotechnical/soil and foundation investigation for the proposed phase 16 and 17 development at Kepayan Ridge, Kota Kinabalu, Sabah Malaysia. Unpublished Geotechnical Report.

Archambault, G., Fortin, M., Gill, D.E., Aurbertin, M., Ladanyi, B., 1990. Experimental investigations for an algorithm simulating the effect of variable normal stiffness on discontinuities shear strength. In: Barton $\mathrm{N}$ and Stephansson $\mathrm{O}$ (Eds), Proceeding of the International Symposium on Rock Joints, Loen, Norway, 4-6 June 1990. Balkema, Rotterdam, pp 141-148.

ASTM D5878-08., 2008. Standard Guides for Using Rock-Mass Classification Systems for Engineering Purposes. ASTM International, West Conshohocken, PA, USA. doi:10.1520/D5878-08.

Ayalew, L., Yamagishi, H., 2005. The application of GIS-based logistic regression for landslide susceptibility mapping in the Kakuda-Yahiko Mountains, Central Japan. Geomorphology 65, 15 - 31.

Aydan, O., Kawamoto, T., 1990. Discontinuities and their effect on rock mass. In: Barton $\mathrm{N}$ and Stephansson $\mathrm{O}$ (Eds), Proceeding of the International Symposium on Rock Joints, Loen, Norway, 4-6 June 1990. Balkema, Rotterdam, pp 149-156.

Bandis, S.C., 1990. Mechanical properties of rock joints. In: Barton $\mathrm{N}$ and Stephansson $\mathrm{O}$ (Eds) Proceeding of the International Symposium on 
Rock Joints, Loen, Norway, 4-6 June 1990. Balkema, Rotterdam, pp 125-140.

Barton, N., 1973. Review of a new shear-strength criterion for rock joints. Engineering Geology 7 (4), 287-332. doi:10.1016/00137952(73)90013-6.

Barton, N., 2013. Shear strength criteria for rock, rock joints, rockfill and rock masses: Problems and some solutions. Journal of Rock Mechanics and Geotechnical Engineering, 5 (4), 249-261. doi: 10.1016/j.jrmge.2013.05.008

Barton, N.R., Lien, R., Lunde, J., 1974. Engineering classification of rock masses for the design of tunnel support. Rock mechanics, 6 (4), 189 236. doi: $10.1007 /$ BF01239496

Barton, N., Choubey, V., 1977. The shear strength of rock joints in theory and practice. Rock Mechanics 10 (1-2), 1-54. doi:10.1007/BF01261801.

Barton N, Stephansson, O., (Eds), 1990. Rock joints; International symposium on rock joints. Loen, Norway, 4-6 June 1990. Balkema, Rotterdam, p 814.

Beauvais, A., Colin, F., 1993. Formation and transformation processes of iron duricrust systems in tropical humid environment. Chemical Geology, 106 (1-2), 77-101. doi: 10.1016/0009-2541(93)90167-H

Begonha, A., Sequeira Braga, M.A., 2002. Weathering of the Oporto granite: geotechnical and physical properties. Catena. 49 (1-2). DOI: 10.1016/S0341-8162(02)00016-4. ISSN: 0341-8162. pp. 57-76.

Berner, R.A., 1984. Sedimentary pyrite formation: An update. Geochimica et Cosmochimica Acta, 48 (4), 605-615. doi: 10.1016/00167037(84)90089-9

Bland, W., Rolls, D., 1998. Weathering: An Introduction to the Scientific Principles. Arnold Publishers, London, $288 \mathrm{pp}$.

Borrelli, L., Greco, R., Gullà, G., 2007. Weathering grade of rock masses as a predisposing factor to slope instabilities: Reconnaissance and control procedures. Geomorphology 87(3), 158-175.

Bouma, A.H., 1962. Sedimentology of some flysch deposits; a graphic approach to facies interpretation. Elsevier Pub. Co, Amsterdam

BS 5930:1981., 1981. Code of practice for site investigations. British Standards Institution, London

BS 5930:1999., 1999. Code of practice for site investigations. British Standards Institution, London

Burgan, A.M., Ali, C. ., 2009. Characterization of the Black Shales of the Temburong Formation in West Sabah, East Malaysia. European Journal of Scientific Research, 30 (1), 79-98.

Calcaterra, D., Parise, M., 2005. Landslide types and their relationships with weathering in a Calabrian basin, southern Italy. Bull Eng Geol Environ 64, $193-207$. 
Chacon, J., Irigaray, C., Fernandez, T., El Homdouni, R., 2006. Engineering geology maps: landslides and geographical information systems. Bulletin of Engineering Geology and the Environment 65, $341-411$.

Che, V.B., Kervyn, M., Suh, C.E., Fontijn, K., Ernst, G.G.J., del Marmol, M.A., Trefois, P., Jacobs, P., 2012. Landslide susceptibility assessment in Limbe (SW Cameroon): A field calibrated seed cell and information value method. Catena 72, 83 - 98.

Chesworth, W. E., 2008. Encyclopedia of Soil Science. Encyclopedia of Earth Sciences Series, 2 Edition. Springer, Dordrecht, The Netherlands.

Chigara, M., 1990. A mechanism of chemical weathering of mudstones in a mountainous area. Engineering Geology 29, 119 - 138. doi: 10.1016/0013-7952(90)90002-i.

Chigira, M., Sone, K., 1991. Chemical weathering mechanisms and their effects on engineering properties of soft sandstone and conglomerate cemented by zeolite in a mountainous area. Engineering Geology, 30 (2), 195-219. doi: 10.1016/0013-7952(91)90043-k.

Chigira, M. \& Oyama, T. 2000. Mechanism and effect of chemical weathering of sedimentary rocks. Engineering Geology, 55 (1-2), 3-14. doi: 10.1016/s0013-7952(99)00102-7.

Chigira, M., Mohamad, Z., Sian, L.C., Komoo, I., 2011. Landslides in weathered granitic rocks in Japan and Malaysia. Bulletin Geological Society of Malaysia 57, 1-6.

Chung, C.F., Fabbri, A.G., 1999. Probabilistic Prediction Models for Landslide Hazard Mapping. Photogrammetric Engineering and Remote Sensing 65 (12), $1389-1399$.

Chung, C.F., Fabbri, A.G., 2003. Validation of Spatial Prediction Models for Landslide Hazard Mapping. Natural Hazards 30, 451 - 472.

Collenette, P., 1958. The geology and mineral resources of the Jesselton Kinabalu area, North Borneo. British Borneo Geological Survey Department, Memoir 6, 194 pp.

Colman, S.M., 1981. Rock-weathering rates as functions of time. Quaternary Research 15 (3), 250-264.

Cruden, D.M., 1977. Describing the size of discontinuities. International Journal of Rock Mechanics and Mining Science \& Geomechanics 14 (3), 133-137. doi:10.1016/0148-9062(77)90004-3.

Cruden, D.M., Varnes, D.J., 1996. Landslide types and processes. In: Turner, K.T., Schuster, R. L., (Eds). Landslides: investigation and mitigation. Transportation Research Board, National research Council, Special Report no. 247, Washington D.C., 36 - 75.

Cuccuru, S., Casini, L., Oggiano, G., Cherchi, G., 2012. Can weathering improve the toughness of a fractured rock? A case study using the San Giacomo granite. Bulletin of Engineering Geology and the Environment, 71 (3), 557-567. doi: 10.1007/s10064-012-0416-9. 
Dahal, R.K., Hasegawa, S., Nonomura, A., Yamanka, M., Masuda, T., Nishino, K., 2008. GIS-based weights of evidence modeling of rainfall-induced landslides in small catchments for landslides susceptibility mapping. Environmental Geology Environ Geology 54, 311 - 324.

Dai, F.C., Lee, C.F., 2001. Terrain-based mapping of landslide susceptibility using a geographical information system: a case study. Canadian Geotechnical Journal 38, 911 - 923.

Dai, F.C., Lee, C.F., 2002. Landslide characteristics and slope instability modelling using GIS, Lantau Island, Hong Kong. Geomorphology 42, $213-228$.

Das, I., Sahoo, S., van Westen, C., Stein, A., Hack, R., 2010. Landslide susceptibility assessment using logistic regression and its comparison with a rock mass classification system, along a road section in the northern Himalayas (India). Geomorphology 114, 627 - 637.

Day, M.J., Leigh, C., Young, A., 1980. Weathering of rock discs in temperate and tropical soils. Zeitschrift fur Gormorphologie Supplementband 35, 11-15.

Department of Town and Country Planning (DTCP), Malaysia., 2009. Guideline on hillside and highland development plan. Ministry of Housing and Local Government, Malaysia, 28p. (in Malay)

De Mulder, E.J.F., Hack, H.R.G.K., Van Ree, C.C.D.F., 2012. Sustainable Development and Management of the Shallow Subsurface. The Geological Society, London.

Dips., 2012. Dips software. Plotting, analysis and presentation of structural data using spherical projection techniques. 5.0 Edition. Rocscience, Toronto, Ontario, Canada.

Dragovich, D., 1987. Measuring stone weathering in cities: Surface reduction on marble monuments. Environmental Geology 9(3), 139-141.

Durgin, P.B., Landslides and the weathering of granitic rocks. Review in Engineering Geology Vol. 111, 127 - 131.

Ehlen, J., 1999. Fracture characteristics in weathered granites. Geomorphology $31 \quad(1-4), \quad 29-45 . \quad$ doi:10.1016/S0169$555 \times(99) 00071-9$.

Ehlen, J., 2002. Some effects of weathering on joints in granitic rocks. CATENA 49 (1-2), 91-109. doi:10.1016/S0341-8162(02)00019-X.

Einstein, H.H., Veneziano, D., Baecher, G.B., O'Reilly, K.J., 1983. The effect of discontinuity persistence on rock slope stability. International Journal of Rock Mechanics and Mining Science \& Geomechanics 20 (5), 227236. doi:10.1016/0148-9062(83)90003-7.

Fell, R., Corominas, J., Bonnard, C., Cascini, L., Leroi, E., Savage, W.Z., 2008. Guidelines for landslide susceptibility, hazard and risk zoning for land use planning. Engineering Geology 102, 85 -98.

Fell, R., MacGregor, P., Stapledon, D., Bell, G., 2012. Geotechnical Engineering of Dams. Taylor \& Francis, New York. 
Fookes, P.G., Gourley, C.S., Ohikere, C., 1988. Rock weathering in engineering time. Quarterly Journal of Engineering Geology 21, 3357.

Frattini, P., Crosta, G., Carrara, A., 2010. Techniques for evaluating the performance of landslide susceptibility models. Engineering Geology $111,62-72$.

Geertsema, A.J., 2003. The shear strength of rock joints with special reference to dam foundations. University of Pretoria, Pretoria, South Africa

GCO, 1990. Foundation Properties of Marble and Other Rocks in the Yuen Long-Tuen Mun Area 2/90. Geotechnical Control Office, Civil Engineering Services Department, Hong Kong. p. 112

Ghazvinian, A., Nikudel, M.R., Sarfarazi, V., 2007. Effect of rock bridge continuity and area on shear behavior of joints. In: Ribeiro e Sousa L, Olalla C, Grossmann NF (Eds), 11th Congress of the International Society for Rock Mechanics: The second half century of rock mechanics, Lisbon, Portugal, 9-13 July 2007. 1, Taylor \& Francis/Balkema, Leiden, pp 247-250.

Ghazvinian, A. H., Taghichian, A., Hashemi, M., Mar'ashi, S. A., 2010. The shear behavior of bedding planes of weakness between two different rock types with high strength difference. Rock Mechanics and Rock Engineering, 43 (1), 69-87. doi: 10.1007/s00603-009-0030-8

Ghiasi, V., Omar, H. \& Huat, B.K., 2009. A Study of the Weathering of the Seremban Granite. The Electronic Journal of Geotechnical Engineering (EJGE) 14 (Bund D), 9 pp.

Glade, T., Crozier, M. J., 2005. The nature of landslide hazard and impact. In: Glade, T., Anderson, M. G., Crozier, M.J., (eds). Landslide Hazard and Risk, Wiley, London, 43 - 74.

Goodman, R.E., 1989. Introduction to rock mechanics. Second Edition. Wiley \& Sons, New York.

Goodman, R.E., Kieffer, D.S., 2000. Behavior of Rock in Slopes. Journal of Geotechnical and Geoenvironmental Engineering 126 (8), 675-684. doi: 10.1061/(asce) 1090-0241 (2000) 126: 8 (675).

Gue, S.S., Tan, Y.C., 2006. Landslides: Abuses of the prescriptive method. In: Proceeding of the International Conferences on Slopes, Malaysia, $13 \mathrm{pp}$.

Gupta, A.S., Rao, K.S., 2000. Weathering effects on the strength and deformational behavior of crystalline rock under uniaxial compression state. Engineering Geology 56, 257-274.

Gupta A.S., Rao, K.S., 2001. Weathering indices and their applicability for crystalline rocks. Bulletin of Engineering Geology and the Environment 60 (3), 201-221. doi:10.1007/s100640100113

Gurocak, Z., Kilic, R., 2005. Effect of weathering on geomechanical properties of the Miocene basalts in Malatya, Eastern Turkey. Bulletin of 
Engineering Geology and the Environment 6, 373-381. doi: 10.1007/s10064-005-0005-2.

Gutiérrez, M., 2005. Climatic Geomorphology. Series: Development in Earth Surface Processes 8, $762 \mathrm{pp}$.

Guzzetti, F., Carrara, A., Cardinali, M., Reichenbach, P., 1999. Landslide hazard evaluation: a review of current techniques and their application in a multi-scale study, Central Italy. Geomorphology 31, 181-216.

Guzetti, F., Reichenbach, P., Ardizzone, F., Cardinalli, M., Galli, M., 2006. Estimating the quality of landslides susceptibility models. Geomorphology 81, $166-184$.

Guzetti, F., Mondini, A. C., Cardinali, M., Fiorucci, F., Santangelo, M., Chang, K-T., 2012. Landslide inventory maps: New tools for an old problem. Earth-Science reviews 112, 42 - 66.

Hachinohe, S., Hiraki, N., Suzuki, T., 1999. Rates of weathering and temporal changes in strength of bedrock of marine terraces in Boso Peninsula, Japan. Engineering Geology 70, 231-237.

Hack, H.R.G.K. 2011. Discontinuous Rock Mechanics : Lecture Notes. University Twente-ITC, The Netherlands.

Hack, H.R.G.K., 1998. Slope Stability Probability Classification. Second Edition PhD Thesis. University of Technology Delft/International Institute for Aerospace Survey and Earth Sciences, ITC, Delft, Enschede, The Netherlands.

Hack, H.R.G.K., 2006. Discontinuous Rock Mechanics. Lecture Notes. Version 5 Edition. ITC, Enschede, The Netherlands, 233 pp.

Hack, H.R.G.K., Price, D.G., 1995. Determination of discontinuity friction by rock mass classification. In: Fujii $T$ (Ed), 8th International Congress on Rock Mechanics, Tokyo, 25-29 September 1995. 3, Balkema, Rotterdam, pp 23-27.

Hack, H.R.G.K., Price, D.G., 1997. Quantification of weathering. In: Marinos PG, Koukis GC, Tsiambaos GC and Stournaras GC (Eds), Engineering Geology and the Environment, Proceedings of an International Symposium of the IAEG, Athens, Greece, 23-27 June 1997, Taylor and Francis Balkema, Rotterdam, The Netherlands, pp.145-150. ISBN: 90-5410-877-0

Hack, H.R.G.K., Huisman, M., 2002. Estimating the intact rock strength of a rock mass by simple means. In: van Rooy JL and Jermy CA (Eds), Engineering geology for developing countries-Proceedings of $9^{\text {th }}$ Congress of the International Association for Engineering Geology and the Environment: South African Institute of Engineering Geologist (SAIEG), Durban South Africa, pp.1971-1977.

Hack, H.R.G.K., Price, D.G., Rengers, N., 2003. A new approach to rock stability - a probability classification (SSPC). Bulletin Engineering 
Geology and the Environment 62, 167-184 and erratum p. 185. doi: $10.1007 / \mathrm{s} 10064-002-0155-4$

Hammah, R.E., Curran, J.H., 2009. It is batter to be approximately right than precisely wrong: why simple models work in mining geomechanics. In: Proceeding $43^{\text {rd }}$ US Rock Mechanics Symposium and $4^{\text {th }}$ U.S. Canada Rock Mechanic Symposium. Asheville, NC, USA. 8pp

Hencher, S.R., Knipe, R.J., 2007. Developments of rock joints with time and consequences for engineering. In: Olalla C, Grossmann N, Ribeiro e Sousa L (Eds), The Second Half Century of Rock Mechanics; Proceedings of the 11th congress of the International Society for Rock Mechanics, Lisbon, 9-13 July 2007. 1, Taylor \& Francis, London, pp 223-226.

Hencher, S.R., Richards, L.R., 1989. Laboratory direct shear testing of rock discontinuities. Ground Engineering, 22 (2), 24-31.

Hencher, S.R., 2008. Eurocodes; The 'new' British and European standard guidance on rock description. Ground Engineering, 41 (7), 17-21.

Hencher, S. R,. McNicholl, D. P., 1995. Engineering in weathered rock. Quarterly Journal of Engineering Geology and Hydrogeology, 28 (3), 253-266. doi: 10.1144/gsl.qjegh.1995.028.p3.04

Hencher., S.R., 2013. Characterizing discontinuities in naturally fractured outcrop analogues and rock core: the need to consider fracture development over geological time. In: Spence, G.H., Redfern, J., Aguilera, R., Bevan, T.G., Cosgrove, J.W., Couples, G.D., \& Daniel, J.M. (Eds). Advances in the Study of Fractured reservoirs. Geological Society of London, Special Publications 374. doi:10.1144/sp374.15.

Heimsath, A.M., Chappell, J., Dietrich, W.E., Nishiizumi, K., Finkel, R.C., 2000. Soil production on a retreating escarpment in Southern Australia. Journal of Geology 28 (19), 787 - 790.

Hack, R., Price, D., Rengers, N., 2003. A new approach to rock stability - a probability classification (SSPC). Bulletin of Engineering Geology and the Environment 62, 167 - 184.

Hervas, J., Bobrowsky, P., 2009. Mapping: Inventories, Susceptibility, Hazard and Risk. In: Sassa, K. and Canutti, P. (Eds). Landslides - Disaster Risk Reduction. Springer, Berlin. pp 321 - 349.

Hinsinger, P., Fernandes Barros, O.N., Benedetti, M.F., Noack, Y., Callot, G., 2001. Plant induced weathering of a basaltic rock: experimental evidence. Geochimica et Cosmochiica Acta 65, 137-152.

Hoek, E. \& Bray, J. 1981. Rock slope engineering. Revised 3rd Edition. Institution of Mining and Metallurgy, London.

Hoek, E., Brown, E.T., 1997. Practical estimates of rock mass strength. International Journal of Rock Mechanics and Mining Science \& Geomechanics Abstracts 34 (8), 1165 - 1186. doi:10.1016/S13651609(97)80069-X. 
Hosmer, D.W., Lameshow, S., 2000. Applied logistic regression. Second Edition, John Wiley and Sons. 375 pp.

Hudson, J.A., Priest, S.D., 1979. Discontinuities and rock mass geometry. International Journal of Rock Mechanics and Mining Science \& Geomechanics 16 (6), 339-362. doi:10.1016/0148-9062(79)900019.

Hudson, J.J., Harrison, J.P., 1997. Engineering Rock Mechanics: an introduction to principles. Pergamon, 444pp.

Huisman, M., Hack, H.R.G.K., 2002. Quantifying slope stability decrease in engineering lifetimes using boothstrap percentiles. In: Dinis da Gama C and Ribeiro e Sousa L (Eds), Proceedings Eurock 2002-I.S.R.M. International Symposium on Rock Engineering for Mountainous Regions, Madeira, Portugal: Publ. Socciedade Portuguesa de Geotecnia, Portugal, 197-204.

Huisman, M., 2006. Assessment of rock mass decay in artificial slopes. PhD Thesis, ITC, Enschede.

Huisman, M., Hack, H.R.G.K., Nieuwenhuis, J.D., 2004. Observed rock mass degradation and resulting slope instability. In: Schubert W (Editions) EUROCK 2004 \& 53rd Geomechanics colloquy: Rock engineering and practice, Salzburg, 7-9 October 2004. Austrian Society for Geomechanics/VGE, Essen, pp 449-452.

Huisman, M., Hack. H.R.G.K., Nieuwenhuis, J.D., 2006. Predicting rock mass decay in engineering lifetimes: the influence of slope aspect and climate. Environmental and Engineering Geoscience 12(1), 39-51. doi: $10.2113 / 12.1 .39$

Hutchison, C.S., 2005. Geology of North-West Borneo; Sarawak, Brunei and Sabah. Elsevier, Amsterdam.

Indraratna, B., Welideniya, H.S., Brown, E.T., 2005. A shear strength model for idealised infilled joints under constant normal stiffness. Géotechnique 55, 215-226. doi:10.1680/geot.2005.55.3.215.

Indraratna, B., Oliveira, D.A.F., Brown, E.T., 2010. A shear-displacement criterion for soil-infilled rock discontinuities. Géotechnique 60, 623633. doi:10.1680/geot.8.P.094.

Indraratna, B., Premadasa, W.N., Oliveira, D.A.F., Welideniya, H.S., 2011. Development of sediment-infilled rock joint models and implications on rockmass stability. In: Khalili, N. \& Oeser, M. (eds) Computer methods for geomechanics : frontiers and new applications ; Proceedings of the 13th International Conference of the International Association for Computer Methods and Advances in Geomechanics (IACMAG), Melbourne, Australia, 9-11 May 2011. University of New South Wales, Centre for Infrastructure Engineering and Safety, Sydney, 290-295.

Inkpen, R.J., Jackson, J., 2000. Contrasting weathering rates in coastal, urban and rural areas in southern Britain: preliminary investigations 
using gravestones. Earth Surface Processes and Landforms 25, 229 238.

Irfan, T.Y., Dearman, W.R., 1978. Engineering petrography of weathered granite. Quarterly Journal of Engineering Geology 11, 233 - 244.

Ismail, A.R., Sanudin, T., Baba, M., Shariff, A.K.O., 2009. Lithological unit thickness approach for determining intact rock strength (IRS) of slope forming rock material of Crocker Formation. Borneo Science 25, 23-32.

ISO 14689-1:2003., 2003. Geotechnical investigation and testing. Identification and classification of rock. Part 1: Identification and description. 1st edition. International Organization for Standardization, Geneva, Switzerland.

ISRM., 1981. Basic Geotechnical Description of Rock Masses. International Journal of Rock Mechanics and Mining Science \& Geomechanics 18, 85-110.

Jade, S., Sarkar, S., 1993. Statistical model for slope instability classifications. Engineering Geology 36, 71 - 98.

Jaiswal, P., van Westen, C,J., Jetten, V., 2010. Quantitative landslide hazard assessment along a transportation corridor in southern India. Engineering Geology 116, 236 - 250.

Jaiswal, P., 2011. Landslide risk quantification along transportation corridors based on historical information. PhD Thesis. University of Twente, Enschede, 243 pp.

Jamaludin, S., Hussien, A.N., 2006. Landslide hazard and risk assessment: The Malaysian experience. 10th IAEG International Congress Nottingham United Kingdom. IAEG Paper No. 455, 10 pp.

Jamaludin, S., Huat, B.B.K., Omar, H., 2006a. Evaluation of Slope Assessment System for Predicting Landslides of Cut Slopes in Granitic and Metasediment Formations. American Journal of Environmental Sciences 2 (4), $135-141$.

Jamaludin, S., Huat, B.B.K., Omar, H., 2006b. Evaluation and Development of Cut-slope Assessment System for Peninsular Malaysia in Predicting Landslide in Granitic Formation. Jurnal Teknologi 44 (B), 31 - 46.

Jamaludin, S., Hussien, A.N., 2006c. Human factors and slope failures in Malaysia. Bulletin Geological Society of Malaysia 52, 75 - 84.

Jeong, U., Yoon, W., Choi, J., Kim, J., 2005. Influence of weathering depth and fracture intensity to cut slope movement. Geoscience Journal 9 (1), 47-52. doi:10.1007/BF02910553.

Karami, A., Stead, D., 2008. Asperity Degradation and Damage in the Direct Shear Test: A Hybrid FEM/DEM Approach. Rock Mechanics and Rock Engineering, 41 (2), 229-266. doi: 10.1007/s00603-007-0139-6.

Katongo, C., 2005. Ground conditions and support systems at 1 shaft, Konkola mine, Chililabombwe, Zambia. The Third Southern African Conference on Base Metals: 'Southern Africa's response to changing 
global base metals market dynamics'. The South African Institute of Mining and Metallurgy, Kitwe, Zambia, pp. 253-280.

Kim, B.H., Cai, M., Kaiser, P.K., 2007. Rock Mass strength with non-persistent joints. In: Eberhardt E, Stead D, Morrison T (Eds) Rock Mechanics: Meeting Society's Challenges and Demands; Proceeding of the 1st Canada-US Rock Mechanics Symposium, Vancouver, Canada, 27-31 May 2007. Taylor \& Francis, New York, pp 241-248.

Komoo, I., 1985. Engineering properties of weathered rock profile in Peninsula Malaysia In: 8th Southeast Asian Geotechnical Conference, Kuala Lumpur, 11-15 March 1985. pp 3.81-86.

Komoo, I., Aziz, S., Sian, L.C., 2011. Incorporating the Hyogo Framework for Action into landslide disaster risk reduction in Malaysia. Bulletin Geological Society of Malaysia 57, 7 - 11.

Kottek, M., Grieser, J., Beck, C., Rudolf, B., Rubel, F., 2006. World Map of the Köppen-Geiger climate classification updated. Meteorologische Zeitschrift 15 (3), 259-263. doi:10.1127/0941-2948/2006/0130.

Kulatilake, P., Um, J., Panda, B., Nghiem, N., 1999. Development of New Peak Shear-Strength Criterion for Anisotropic Rock Joints. Journal of Engineering Mechanics 125 (9), 1010-1017. doi:10.1061/(ASCE)0733-9399(1999)125:9(1010).

Kulatilake, P.H.S.W., Shou, G., Huang, T.H., Morgan, R.M., 1995. New peak shear strength criteria for anisotropic rock joints. International Journal of Rock Mechanics and Mining Sciences \& Geomechanics Abstracts, 32 (7), 673-697. doi: 10.1016/0148-9062(95)00022-9

Laubscher, D.H., 1990. A geomechanics classification system for rating of rock mass in mine design. Journal of the South African Institute of Mining and Metallurgy, 90 (10), 257-273.

Lee, S.-G., Hencher, S.R., 2009. The repeated failure of a cut-slope despite continuous reassessment and remedial works. Engineering Geology, 107 (1-2), 16-41. doi: 10.1016/j.enggeo. 2009.03.011

Lee, C.H., Lee, M.S., Suh, M., Choi, S.W., 2005. Weathering and deterioration of rock properties of the Dabotap pagoda (World Cultural Heritage), Republic of Korea. Environmental Geology 47, 547 - 557.

Lee, S., Talib, A.T., 2005. Probabilistic landslide susceptibility and factor effect analysis. Environmental Geology 47, 982 - 990.

Lemy, F., Hadjigeorgiou, J., 2003. Discontinuity trace map construction using photographs of rock exposures. International Journal of Rock Mechanics and Mining Science \& Geomechanics 40 (6), 903-917. doi:10.1016/S1365-1609(03)00069-8

Liesegang, R.E., 1896. Ueber einige Eigenschaften von Gallerten. Naturwissenschaftliche Wochenschrift, 11 (30), 353-362.

Lin, M.L., Tung, C.C., 2003. A GIS-based potential analysis of the landslides induced by the Chi-Chi earthquake. Engineering Geology 71, 63-77. 
Magliulo, P., Di Lisio, A., Russo, F., Zelano, A., 2008. Geomorphology and landslide susceptibility assessment using GIS and bivariate statistics: a case study in southern Italy. Natural Hazards 47, 411 - 435

Malamud, B.D., Turcotte, D.L., Guzetti, F., Reichenbach, P., 2004. Landslide inventories and their statistical properties. Earth Surface Processes and Landforms 29, 687 - 711.

Marques, E.A.G., Barroso, E.V., Menezes Filho, A.P., Vargas Jr, E.d.A., 2010. Weathering zones on metamorphic rocks from Rio de JaneiroPhysical, mineralogical and geomechanical characterization. Engineering Geology. 111 (1-4). DOI: 10.1016/ j.enggeo. 2009. 11. 001. ISSN: 0013-7952. pp. 1-18.

Mezughi, T.H., Mat Akhir, J., Rafek, A.G., Abdullah, I., 2011a. Landslide susceptibility assessment using frequency ratio model applied to an area along the E - W Highway (Gerik - Jeli). American Journal of Environmental Sciences 7 (1), 43 - 50.

Mezughi, T., Mat Akhir, J., Rafek, A.G., Abdullah, I., 2011b. A multi-class weight of evidence approach for landslide susceptibility mapping applied to an area along the E - W Highway (Gerik - Jeli), Malaysia. The Electronic Journal of Geotechnical Engineering Vol. 16 Bund. O, 1259 - 1273.

McConaughy, D.T., Engelder, T., 2001. Joint initiation in bedded clastic rocks. Journal of Structural Geology 23 (2-3), 203-221. doi:10.1016/S0191-8141(00)00091-2.

McKibben, M.A., Barnes, H.L., 1986. Oxidation of pyrite in low temperature acidic solutions: Rate laws and surface textures. Geochimica et Cosmochimica Acta, 50 (7), 1509-1520. doi: 10.1016/00167037(86)90325-x.

McNeill, G.W., 1999. Variations in the weathering rate of Scottish gravestones as an environmental signature of atmospheric pollution. Environmental Geochemistry and Health 21(4), 365-370.

Miscevic, P., Vlastelica, G., 2014. Impact of weathering on slope stability in soft rock mass. Journal of Rock Mechanics and Geotechnical Engineering 6, $240-250$.

ML Geoscience Services., 2008. Soil Investigation for the proposed condominium development on CL015000599 at Jalan Bukit Bendera, Kota Kinabalu, Sabah, Malaysia. Unpublished Geotechnical Report.

MMD., 2013. General Climate of Malaysia, Malaysian Meteorological Department, Ministry of Science, Technology and Innovation. http://www.met.gov.my/index.php?lang=english. Accessed 9 June 2013.

Mohamad, E.T., Isa, M.F.M., Amin, M.F.M., Komoo, I., Gofar, N., Saad, R., 2011. Effect of moisture content on the strength of various weathering grades of granite. The Electronic Journal of Geotechnical Engineering (EJGE) 16 (Bund H), 863 - 886. 
Mohamad, E.T., Komoo, I., Kassim, K.S., Gofar, N., 2008. Influence of moisture content on the strength of weathered sandstone. Malaysian Journal of Civil Engineering 20 (1), 137-144.

Mohamed, Z., Rafek, A.G., Komoo, I., 2007. Characterization and classification of the physical deterioration of Tropically weathered Kenny Hill rock for civil works. The Electronic Journal of Geotechnical Engineering (EJGE) 12 (Bund A), 16pp.

Nichiyama, K., Matsukura, Y., 2006. Weathering rates and mechanisms causing changes in rock properties of sandstone. In: Proceeding $10^{\text {th }}$ IAEG International Congress, Nottingham UK, Paper No. 278, 6pp.

Nicholson, R.V., Gillham, R.W., Reardon, E.J., 1988. Pyrite oxidation in carbonate-buffered solution: 1. Experimental kinetics. Geochimica et Cosmochimica Acta, 52 (5), 1077-1085. doi: 10.1016/00167037(88)90262-1.

Nicholson, D.T., 2004. Hazard assessment for progressive, weathering-related breakdown of excavated rockslopes. Quarterly Journal of Engineering Geology and Hydrogeology 37, 327-346.

Niton XL3t, 2013. Thermo Scientific Niton XL3t XRF Analyzer. Thermo Scientific Portable Analytical Instruments Inc., Tewksbury, MA, USA. http://www.niton.com/en/. Accessed 25 June 2013.

Ollier, C.D., 1969. Weathering. Oliver and Boyd. 304 pp.

Olvmo, M., 2010. Review of denudation processes and quantification of weathering and erosion rates at a 0.1 to $1 \mathrm{Ma}$ time scale. Technical Report TR-09-18. Svensk Kärnbränslehantering AB; Swedish Nuclear Fuel and Waste Management Co, Stockholm, Sweden, p. 56.

Orhan, M., Işık, N.S., Topal, T., Özer, M., 2006. Effect of weathering on the geomechanical properties of andesite, Ankara - Turkey. Environmental Geology 50 (1), 85-100. doi:10.1007/s00254-0060189-1.

Palmstrøm, A., 1975. Characterization of degree of jointing and rock mass quality. Ing.A.B. Berdal A/S, Oslo, Norway.

Papaliangas, T., Lumsden, A.C., Manolopoulou, S., Hencher, S.R., 1990. Shear strength of modeled filled rock joints. In: Barton, N. R. \& Stephansson, O. (eds) Proceeding of the International Symposium on Rock Joints, Loen, Norway, 4-6 June 1990. Balkema, Rotterdam, 275-282.

Papaliangas, T., Hencher, S.R., Lumsden, A.C., Manolopoulou, S., 1993. The effect of frictional fill thickness on the shear strength of rock discontinuities. International Journal of Rock Mechanics and Mining Science \& Geomechanics 30 (2), 81-91. doi:10.1016/01489062(93)90702-F.

Patton, F.D., 1966. Multiple Modes of Shear Failure In Rock. In: Rocha M (Eds) 1st International Society for Rock Mechanics (ISRM) Congress, Lisbon, Portugal, 25 September-1 October 1966. International 
Society for Rock Mechanics/LNEC (National Laboratory for Civil Engineering), Lisbon, Portugal, pp 509-513.

Pauzi, N.I.M., Omar, H., Bujang, K.H., Misran, H., Yusoff, Z.M., 2011. Microcrack pattern propagations and rock quality designation of Batu Caves Limestone. The Electronic Journal of Geotechnical Engineering (EJGE) 16 (Bund E), 591 - 604.

Pereira, J.P., 1990. Shear strength of filled discontinuities. In: Barton N, Stephansson $\mathrm{O}$ (Eds), Proceeding of the International Symposium on Rock Joints, Loen, Norway, 4-6 June 1990. Balkema, Rotterdam, pp 283-287.

Phien-wej, N., Shrestha, U.B., Rantucci, G., 1990. Effect of infill thickness on shear behavior of rock joints. In: Barton N, Stephansson O (Eds), Proceeding of the International Symposium on Rock Joints, Loen, Norway, 4-6 June 1990. Balkema, Rotterdam, pp 289-294.

Pickles, A., 2005. Rock mass classification for pile foundations. In: The Characterization of Rock Masses for Engineering Purposes, City University, Hong Kong, 25 June 2005. The Geological Society, Hong Kong Regional Group, Hong Kong, p. 36 slides.

Pollard, D.D., Aydin, A., 1988. Progress in understanding jointing over the past century. Geological Society of America Bulletin 100 (8), 11811204.

Pourghasemi, H.R., Pradhan, B., Gokceoglu, C., Moezzi, K.D., 2012. In: B. Pradhan and M. Buchroithner (Eds), Terrigenous Mass Movements: Detection, Modelling, Early Warning and Mitigation Using Geoinformation Technology, Springer Science \& Business Media. $406 p p$.

Pradhan, B., Lee, S., 2008. Utilization of optical remote sensing data and GIS tools for regional landslide hazard analysis by using an artificial neural network model at Selangor, Malaysia. Earth Science Front 14 (6), 143-152.

Pradhan, B., Lee, S., 2010a. Delineation of landslide hazard areas on Penang Island, Malaysia, by using frequency ratio, logistic regression, and artificial neural network models. Environ Earth Sciences 60, 10371054.

Pradhan, B., Lee, S., 2010b. Regional landslide susceptibility analysis using back-propagation neural network model at Cameron Highland, Malaysia. Landslides 7, 13 - 30.

Pradhan, B., Sezer, E.A., Gokceoglu, C., Buchroithner, M.F., 2010c. Landslide susceptibility mapping by neuro-fuzzy approach in a landslide-prone area (Cameron Highlands, Malaysia). IEEE Transactions on Geoscience and Remote Sensing 48, 4164-4177.

Price, D.G., 1995. Weathering and weathering processes. Quarterly Journal of Engineering Geology 28, 243-252. 
Price, D.G., De Freitas, M. H., Hack, H. R. G. K., Higginbottom, I. E., Knill, J. L., Maurenbrecher, M., 2009. In: De Freitas, M. H. (Eds.) Engineering Geology - Principles and Practice. Springer-Verlag, Berlin, Heidelberg. 450pp. doi:10.1007/978-3-540-68626-2.

Price, N.J., 1990. Fault and Joint development in brittle and semi-brittle rock. Pergamon Press, Oxford, UK.

Priest, S.D., 2004. Determination of Discontinuity Size Distributions from Scanline Data. Rock Mechanic and Rock Engineering 37 (5), 347-368. doi:10.1007/s00603-004-0035-2.

Priest, S.D., Hudson, J.A., 1976. Discontinuity spacings in rock. International Journal of Rock Mechanics and Mining Science \& Geomechanics 13 (5), 135-148. doi:10.1016/0148-9062(76)90818-4.

Priest, S.D., Hudson, J.A., 1981. Estimation of discontinuity spacing and trace length using scanline surveys. International Journal of Rock Mechanics and Mining Science \& Geomechanics 18 (3), 183-197. doi: 10.1016/0148-9062(81)90973-6.

Public Works Department, Malaysia (PWD)., 2009. National Slope Master Plan 2009 - 2023. Jabatan Kerja Raya, Malaysia. Kuala Lumpur.

Public Works Department Malaysia (PWD)., 2010. Guidelines for Slope Design. Slope Engineering Branch, Public Works Department, Malaysia. 31pp.

Qasim, S., Harahap, I.S.H., Osman, S.B.S., 2013. Causal Factors of Malaysian Landslides: A Narrative Study. Research Journal of Applied Science, Engineering and Technology 5 (7), 2303 - 2308.

Raiswell, R., Canfield, D. E., 1998. Sources of iron for pyrite formation in marine sediments. American Journal of Science, 298 (3), 219-245. doi: 10.2475/ajs.298.3.219.

Raiswell, R., Canfield, D.E., Berner, R.A., 1994. A comparison of iron extraction methods for the determination of degree of pyritisation and the recognition of iron-limited pyrite formation. Chemical Geology, 111 (1-4), 101-110. doi: 10.1016/0009-2541(94)90084-1.

Raj, J.K., 1985. Characterization of the weathering profile developed over a porphyritic biotite granite in Peninsula Malaysia. Bulletin International Association of Engineering Geology 32, 121-129.

Rawnsley, K.D., Hencher, S.R., Lumsden, A.C., 1990. Joint origin as a predictive tool for the estimation of geotechnical properties. In: Barton N, Stephansson O (Eds), Proceeding of the International Symposium on Rock Joints, Loen, Norway, 4-6 June 1990. Balkema, Rotterdam, pp 91-96.

Razak, K.A., Santangelo, M., van Westen, C.J., Straatsma, M.W., de Jong, S.M., 2013. Generating an optimal DTM from airborne laser scanning data for landslide mapping in a tropical forest environment. Geomorphology 190, 112 - 125. 
Razak, K.A., 2014. Airborne laser scanning For forested landslides investigation In temperate and tropical environments. PhD Thesis. University of Twente/University of Utrecth, Enschede, 184 pp.

Reissmüller, M., 1997. In: Eberhardt, E., Thuro, K., Luginbuehl, 2005. Slope instability mechanisms in dipping interbedded conglomerates and weathered marls: the 1999 Rufi landslide, Switzerland. Engineering Geology. 77 (1-2). DOI: 10.1016/j.enggeo.2004.08.004. pp. 35-56.

Rimstidt, J.D., Vaughan, D.J., 2003. Pyrite oxidation: a state-of-the-art assessment of the reaction mechanism. Geochimica et Cosmochimica Acta, 67 (5), 873-880. doi: 10.1016/s0016-7037(02)01165-1.

Rodeano, R., Tahir, S., Omang, S.A.K.S., 2006. Engineering Geology of the Kota Kinabalu Area, Sabah, Malaysia. Bulletin of the Geological Society of Malaysia 52, $17-25$.

Rodeano, R., Jamaluddin, T.A., Talip, M.A., 2011a. Application of GIS in landslide risk assessment (LRA): A case study of the Kota Kinabalu area, Sabah, Malaysia. Bulletin of the Geological Society of Malaysia $57,69-83$.

Rodeano, R., Jamaluddin, T.A., Talip, M.A., Hassan , S., 2011b. Landslide Hazard Factors (LHF) by Community perception survey in Kota Kinabalu, Sabah. Borneo Science 29: 32 - 45.

Rodeano, R., Jamaluddin, T.A., Talip, M.A., 2012a. Integration of GIS using Geostatistical Interpolation Techniques (Kriging) (GEOSTAINT-K) in Deterministic Models for Landslide Susceptibility Analysis (LSA) at Kota Kinabalu, Sabah, Malaysia. Journal of Geography and Geology, Vol 4 (1), 18 - 32.

Rodeano, R., Jamaluddin, T.A., Talip, M.A., 2012b. Intergration of GIS in Deterministic Model (Infinite Slope) (DESSISM) for Landslide Susceptibility Analysis (LSA) at Kota Kinabalu area, Sabah, Malaysia. Proceeding of National Geosciences Conference 2012, Sarawak Malaysia. 14 pp.

Rodeano, R., Jamaluddin, T.A., Talip, M.A., 2012c. Landslide Susceptibility Mapping (LSM) at Kota Kinabalu, Sabah Malaysia using Factor Analysis Model (FAM). Journal of Advanced Science and Engineering Research 2, 80 - 103.

Rodeano, R., Sanudin, T., Abd Kadir, S.O., 2006. Engineering Geology of the Kota Kinabalu Area, Sabah, Malaysia. Bulletin Geological Society of Malaysia 52, 17-25.

Ruiz-Agudo, E., Putnis, C.V., Rodríguez-Navarro, Y.C., 2007. Role of chemical weathering in salt decay of ornamental stone. In: Proc. MACLA 7, XXVII Reunión De La Sociedad Española De Mineralogía. 11-14 September 2007. Jaén, Spain, pp. 29 - 29.

Ruxton, B.P., 1968. Measures of the degree of chemical weathering of rocks. Journal of Geology 6, 518-527. 
Sasaki, K., Tsunekawa, M., Ohtsuka, T., Konno, H., 1998. The role of sulfuroxidizing bacteria Thiobacillus thiooxidans in pyrite weathering. Colloids and Surfaces A: Physicochemical and Engineering Aspects, 133 (3), 269-278. doi: 10.1016/s0927-7757(97)00200-8.

Sancho, C., Fort, R., Belmonte, A., 2003. Weathering rates of historic sandstone structures in semiarid environments (Ebro basin, NE Spain). Catena 53 (1), 53-64.

Saunders, M.K., Fookes, P.G., 1970. A review of the relationship of rock weathering and climate and its significance to foundation engineering. Engineering Geology 4, 289 - 325.

Schultz, R.A., Fossen, H., 2008. Terminology for structural discontinuities. American Association of Petroleum Geologists Bulletin 92 (7), 853 867. doi:10.1306/02200807065.

Scheel, A., 2009. Robustness of Liesegang patterns. Nonlinearity, 22 (2), 457-483. doi: 10.1088/0951-7715/22/2/012.

Sebastián, E., Cultrone, G., Benavente, D., Fernandez, L.L., Elert, K., Rodriguez-Navarro, C., 2008. Swelling damage in clay-rich sandstones used in the church of San Mateo in Tarifa (Spain). Journal of Cultural Heritage 9, 66-76.

Seidel, JP., Haberfield, C.M., 1995. Towards an understanding of joint roughness. Rock Mechanics Rock Engineering 28 (2), 69-92. doi:10.1007/BF01020062.

Selby, M.J., 1993. Hillslope Materials and Processes, 2nd Edition. Oxford University Press, Oxford, UK, 415 pp.

Semhi, K., Amiotte Suchet, P., Clauer, N., Probst, J.L., 2000. Impact of nitrogen fertilizers on the natural weathering erosion processes and the fluvial transport in the Garonne basin. Applied Geochemistry 15, $865-878$.

Sen, Z., Kazi, A., 1984. Discontinuity spacing and RQD estimates from finite length scanlines. International Journal of Rock Mechanics and Mining Science \& Geomechanics 21 (4), 203-212. doi:10.1016/01489062(84)90797-6.

Siedel, H., Pfefferkorn, S., v. Plehwe-Leisen, E., Leisen, H., 2010. Sandstone weathering in tropical climate: Results of low-destructive investigations at the temple of Angkor Wat, Cambodia. Engineering Geology 115, 182 - 192 .

Sidle, R.C., Ochiai, H., 2006. Landslides: processes, prediction, and land use. Water Resources Monograph 18, American Geophysical Union, Washington, DC, $312 \mathrm{p}$.

Sidle, R.C., Ziegler, A.D., Negishi, J.N., Nik, A.R., Siew R, Turkelboom, F., 2006. Erosion processes in steep terrain - Truths, myths, and uncertainties related to forest management in Southest Asia. Forest Ecology and Management 224, 199-225. 
Singh, H., Huat, B.B.K., Jamaludin, S., 2008. Slope Assessment System: A Review and Evaluation of Current Techniques Used for Cut Slopes in the Mountainous Terrain of West Malaysia. The Electronic Journal of Geotechnical Engineering (EJGE) Vol. 13 Bundle E, 24 pp.

Slob, S., 2010. Automated rock mass characterisation using 3-D terrestrial laser scanning. University of Delft; ITC, Delft/Enschede, The Netherlands.

Soeters, R., van Westen, C. J., 1996. Slope instability recognition analysis and zonation. In: Turner, K. T., Schuster, R. L., (eds). Landslides: investigation and mitigation. Transportation Research Board, National research Council, Special Report no. 247, Washington D.C. 129 177.

Snee, C., 2008. Engineering Geology and cavern design for New York City. In: Roach, M.F., Kritzer, M.R., Ofiara, D. \& Townsend, B.F. (Eds) 9th North American Tunnelling. NAT 2008, San Francisco, 8-11 June 2008. Society for Mining, Metallurgy \& Exploration, Littleton, CO, USA. ISBN: 9780873352635, pp. 364-372.

Stephens, E.A., 1956. The geology and mineral resources of the Kota Belud and Kudat Area, North Borneo, Memoir 5. Geological Survey Department, British Territories in Borneo. Kuching, Sarawak : Printed at the Govt. Print. Off., E.N. Brown, acting govt. printer, Sarawak.

State Library \& IT Centre-Geotechnical Report, 1997. Geotechnical Report for Proposed Sabah State Library Headquarters and Information Technology Centre, Kota Kinabalu, Sabah. STL Geotechnical Company, Kota Kinabalu, Sabah, Malaysia 36.

STL Geotechnical Engineering., 1997. Geotechnical report for proposed Sabah State Library Headquarters and Information Technology Centre, Kota Kinabalu, Sabah. Unpublished Geotechnical Report.

Storemyr, P., 2011. Cleopatra's Needle: Egyptian concern about the NYC obelisk. Per Storemyr Archaeology \& Conservation. http://perstoremyr.net/2011/01/10/cleopatras-needle-egyptian-concern-aboutthe-nyc-obelisk/.

Suzen, M.L., Doyuran, V., 2004. Data driven bivariate landslide susceptibility assessment using geographical information systems: a method and application to Asarsuyu catchment, Turkey. Engineering Geology 71, $303-321$.

Tating, F.F. 1997. Assessment of Quarry Site at Lease No. 025310141 Kampung Lengan Papar. Rep. no GSJL 017/1088/97. Geological Survey Department, Kota Kinabalu, Malaysia.

Tating, F., Hack, R., Jetten, V., 2013a. Engineering aspects and time effects of rapid deterioration of sandstone in the tropical environment of Sabah, Malaysia. Engineering Geology 159, 20-30. doi:10.1016/j.enggeo. 2013.03.009. 
Tating, F., Hack, R., Jetten, V., 2013b. Weathering and deterioration as quantitative factors in slope design in humid tropical areas: case study Northern Kota Kinabalu, Sabah, Malaysia. Ingeokring Newsletter, Engineering in Exotic Environment, Summer 2013, 22 28.

Tating, F., Hack, R., Jetten, V., 2013c. Quantification of rock mass deterioration processes for cut slope design in humid tropical areas case study Northern Kota Kinabalu, Sabah, Malaysia. In: Proceeding National Geoscience Conference, Ipoh, 8 - 9 June 2013, Geological Society of Malaysia, $102-104$.

Tating, F.F., Hack, R. \& Jetten, V. 2015a. Weathering effects on discontinuity properties in sandstone in a tropical environment: Case Study at Kota Kinabalu, Sabah Malaysia. Bulletin of Engineering Geology and the Environment. doi: 10.1007/s10064-014-0625-5.

Tating, F.F., Hack, R. \& Jetten, V. 2015b. Influence of weathering-induced iron precipitation on properties of sandstone in a tropical environment. Submitted to Quarterly Journal of Engineering Geology and Hydrology, 17p.

Tating, F.F., Hack, H.R.G.K. \& Jetten, V., 2015c. Landslide susceptibility assessment using information value statistical method: a case study at northern Kota Kinabalu, Sabah Malaysia. The Malaysian Journal of Remote sensing and GIS. Vol. 4 (2). p. 18

Tating, F.F., 2003. The geology and landslide in the northern Kota Kinabalu, Sabah Malaysia. MSc Thesis. Kumamoto University . Kurokami Kumamoto, Japan (Unpublished).

Thiery, Y., Malet, J.-P., Sterlacchini,S., Puissant, A., Maquaire, O., 2007. Landslide susceptibility assessment by bivariate methods at large scales: Application to a complex mountainous environment. Geomorphology 92, $38-59$.

Tongkul, F., 1989. Weak zones in the Kota Kinabalu Area, Sabah, East Malaysia. Sabah Society Journal, Vol IX (1): $11 \mathrm{pp}$.

Tongkul, F., 1987. Sedimentology and structure of the Crocker Formation in the Kota Kinabalu area, Sabah, East Malaysia. University of London, UK. Unpublished Phd. Thesis. 318 pp.

Tongkul, F., 1990. Structural style and tectonics of Western and Northern Sabah. Bulletin of Geological Society of Malaysia 27, 227 - 239.

Taylor, H.W., 1980. A geomechanics classification applied to mining problems in the Shabanie and King Chrysotile asbestos mines, Rhodesia. M.P., University of Rhodesia, Harare, Zimbabwe.

Tay, L.T., Sh. Alkhasawneh, M., Lateh, H., Md. Hossain, K., Kamil, A.A., 2014. Landslide hazard mapping of Penang Island using Poisson Distribution with dominant factors. Journal of Civil Engineering Research 4 (3A), 72 -77. doi: 10.5923/c.jce.201402.12. 
Tuğrul, A., 2004. The effect of weathering on pore geometry and compressive strength of selected rock types from Turkey. Engineering Geology 75 (3-4), 215-227. doi:10.1016/ j.enggeo. 2004. 05. 008.

Ulusay, R., Hudson, J.A. (Eds)., 2007. The Blue Book; The Complete ISRM Suggested Methods for Rock Characterization, Testing and Monitoring: 1974-2006. Commission on Testing Methods ISRM. International Society for Rock Mechanics (ISRM), Turkish National Group, Ankara, Turkey.

Ündül, Ö., Tuğrul, A., 2011. The Influence of Weathering on the Engineering Properties of Dunites. Rock Mechanics Rock Engineering 45 (2), 225239. doi:10.1007/s00603-011-0174-1.

Van Hattum, M.W.A., Hall, R., Nichols, G. J., 2003. Provenance of Northern Borneo Sediments. In: 29th IPA Annual Convention Proceedings, Jakarta, 14-16 October 2003. 1, Indonesian Petroleum Association (IPA), Jakarta, 305-319.

van Westen, C.J., 1997. Statistical landslide hazard analysis. ILWIS 2.1 for Windows application guide. ITC Publication, Enschede, The Netherlands, pp 73-84.

van Westen, C.J., Rengers, N., Soeters, R., 2003. Use of Geomorphological Information in Indirect Landslide Susceptibility Assessment. Natural Hazards 30, 399 - 419.

van Westen, C.J., Castellos, E., Kuriakose, S.L., 2008. Spatial data for landslide susceptibility, hazard, and vulnerability assessment: An overview. Engineering Geology 102, $112-131$.

Varnes, D.J., 1978. Slope movement: types and process. In: Schuster, R.L., and Krizek, R.J., (Eds). Landslides: Analysis and Control. Transportation Research Board, National research Council, Special Report no. 176, Washington D.C., $11-33$.

Viles, H.A., 2013. Linking weathering and rock slope instability: non-linear perspectives. Earth surface processes and landforms 38, $62-70$.

Vincente Silvestre, M.V., Ribeiro e Sousa, L., Hack, R., 2002. Laboratory study of geometrical and hydro-mechanical characteristics of discontinuities. ISRM News Journal, 7 (2), 9-15, 23.

von Routte, J., Papritz, A., Lehman, P., Rickli, C., Or, D., 2011. Spatial statistical modeling of shallow landslides-Validating predictions for different landslide inventories and rainfall events. Geomorphology 133, $11-22$.

Warke, P.A., McKinley, J., Smith, B.J., 2006. Weathering of building stone: approaches to assessment, prediction and modeling. In: S.K. Kourkoulis (Ed) Fracture and Failure of Natural Building Stones. Springer, pp. $313-327$.

Wells, T., Hancock, G., Fryer, J., 2008. Weathering rates of sandstone in a semi-arid environment (Hunter Valley, Australia). Environmental Geology 54(5), 1047-1057. 
Wieczorek, G.F., 1984. Preparing a detailed landslide-inventori map for hazard evaluation and reduction. Bulletin Association Engineering Geologists 21 (3), 337 - 342.

White, A.F., Blum, E.A., 1995. Effects of climate on chemical weathering in watersheds. Geochimica et Cosmochimica Acta 59 (9), 1729 -1747.

White, A.F., Blum, A.E., Schulz, M.S., Vivit, D.V., Stonestrom, D.A., Larsen, M., Murphy, S.F., Eberl, D., 1998. Chemical weathering in a tropical watershed, Luquillo Mountains, Puerto Rico: I. long-term versus short-term weathering fluxes. Geochimica et Cosmochiica Acta 62, 209-226.

Williams, R.B.G., Robinson, D.A., 2000. Effects of aspect on weathering: anomalous behaviour of sandstone gravestones in southeast England. Earth Surface Processes and Landforms 25, 135 - 144.

Wyllie, D.C., Mah, C.W., 2004. Rock slope engineering: civil and mining (based on Rock Slope Engineering by Hoek, E. and Bray, J.). 4th Edition. Spon Press, London; New York.

Winkler, E.M., 1980. Historical Implications in the complexity of destructive salt weathering: Cleopatra's Needle, New York. Bulletin of the Association for Preservation Technology XII (2), 94 -102.

Wong, H.N., Ho, K.K.S., Pun, W.K., Pang P.L.R., 1998. Observations from some landslide studies in Hong Kong. In: Li, K.S., Kay, J.N. \& Ho, K.K.S. (Eds), Slope Engineering In Hong Kong; Annual Seminar Geotechnical Division, Hong Kong Institution of Engineers. Balkema, Rotterdam, pp. 277-286.

WP/WLI (International Geotechnical Societies' UNESCO Working Party for World Landslide Inventory)., 1990. A suggested method for reporting a landslide. Bulletin International Association of Engineering Geology $41,5-12$.

Yalcin, A., 2008. GIS-based landslide susceptibility mapping using analytical hierarchy process and bivariate statistics in Ardesen (Turkey): comparisons of results and confirmations. Catena 72, $1-12$.

Yang, Z. Y., Di, C. C., Yen, K. C., 2001. The effect of asperity order on the roughness of rock joints. International Journal of Rock Mechanics and Mining Sciences, 38 (5), 745-752. doi: 10.1016/S13651609(01)00032-6.

Yin, K. J., Yan, T. Z., 1988. Statistical prediction model for slope instability of metamorphosed rocks. In: Bonnard C (ed.), Landslides. Proceedings of the 5th International Symposium on Landslides, Balkema, Rotterdam, 1269 - 1272.

Yokota, S., Iwamatsu, A., 1999. Weathering distribution in a steep slope of soft pyroclastic rocks as an indicator of slope instability. Engineering Geology 55, 57-68.

Zauyah, S., Stoops, G., 1990. A study of the ferrallitic weathering of an amphibole schist in Peninsula Malaysia. Pertanika 13(1), 85 - 93. 
Zare, M., Kakaie, R., Torabi, S.R., Jalali, S.M.E., 2008. A new empirical criterion for prediction of the shear strength of natural infilled rock joints under constant normal load (CNL) conditions. In: Majdi, A. (ed) 5th Asian Rock Mechanics Symposium; (ARMS5); ISRM International Symposium 2008, Tehran, Iran, 24-26 November 2008. Curran Associates, Inc., Red Hook, NY, USA, 543-550.

Zezere, J.L., 2002. Landslide susceptibility assessment considering landslide typology. A case study in the area north of Lisbon (Portugal). Natural Hazards and Earth Sciences 2, 73 - 82. 


\section{Summary}

Deterioration processes, especially weathering is one of the main causes for slope failures in man-made slopes. Weathering will progressively affect the durability of man-made slopes by decreasing intact rock strength, increasing the number of discontinuities, formation of new discontinuities in intact rock, and decreasing shear and tensile strength along discontinuities. These effects are complemented by the effect from stress relief, as a result of overburden removal or slope excavation.

Weathering and stress relief effects are often prominently present along transportation corridors shown by failures of slopes before the intended "engineering lifetime" ends. Even though, the importance of different weathering grades at the time of excavation has been acknowledged and taken into consideration in slope design criteria, future degradation of slope material has not been anticipated and is often neglected. The reasons are related to the question "how to incorporate future deterioration (particularly weathering and slope relief) into slope design". In order to answer this question there is a need to establish the relationship between deterioration processes and exposure time, which is related particularly to the weathering intensity rate. Subsequently, quantitative factors for weathering to incorporate in slope design have to be developed including weathering effects such as the development of new discontinuities and the change in condition of discontinuities. Based on the relationship between deterioration processes and exposure time information; man-made slope excavation time can be used as a parameter map in the development of landslide susceptibility maps.

This research presents the result of the above-mentioned development of quantitative factors for a particular type of rock (thick - very thick bedded Sandstone unit) in a humid tropical environment at northern Kota Kinabalu, Sabah Malaysia. Characterization of the rock units is based on an extensive and detailed field observations coupled with simple physical testing on intact rock strength in the field. The rock mass was delineated into several geotechnical units based on bedding thickness, lithological type, bedding thickness ratio between different lithology, and weathering grade. Weathering grades of rock masses are determined visually based on BS 5930 (1981). Characterization per geotechnical unit is done in quantified values based on Slope Stability Probability Classification (SSPC) methodology (Hack et al., 2003). Intact rock strength is estimated by using "simple means" method, which used hand and geological hammer based on IRS estimation procedure in BS 5930 (1981) and BS 5930 (1999). 
The quantitative factors development is based on the changes of intact rock strength (IRS) value per unit time within the engineering time due to weathering processes, which is also defined as "weathering intensity rate". The intact rock strength value changes with exposure time decreases nonlinearly with time and is best portrayed by a logarithmic function:

$$
I R S_{t}=105+34 \log (1+t)
$$

$I R S_{t}$ (in $\mathrm{MPa}$ ) is the intact rock strength of sandstone at the time $(t), t$ is the time since exposure in years. The constant 105 is the initial value of the IRS in MPa of the fresh SST unit at the time of excavation (i.e. at exposure time 0 year), and 34 is the apparent reduction rate in $\mathrm{MPa} /(\log$ [year]) for the SST unit.

Similarly, the prediction of future geotechnical parameters (i.e. cohesion and friction angle) can be done by utilizing an empirical equation for rock mass stability such as in Slope Stability Probability Classification (SSPC) method. Thus, better man-made slope design can be done by anticipating the reduction of the geotechnical parameters at particular lifetime.

To evaluate the effect of weathering on the development of discontinuities in rock, the rock mass discontinuity geometry (orientation, spacing, and persistence) and condition of discontinuity (roughness and infill) are organized based on weathering grade and analysed using the Fisher distribution in Dips software. It is found that three dominant sets of discontinuities have been developed in the SST unit due to the weathering processes, the spacing for all discontinuity sets decreases with increase in weathering grade due to the formation of new discontinuities from integral/incipient discontinuities into mechanical discontinuities, and the development of new discontinuities is sequential based on the weathering grade.

In tropical areas, chemical weathering processes (such as oxidation and dissolution) will result in the formation of oxidation and dissolved zones. These zones can be distinguished based on their visual appearance (colour) and presence of iron precipitation. The oxidation zone is brownish to brownish-grey in colour and iron precipitation is very common, whereas the dissolved zone is greyish in colour and iron precipitation is less. Three types of iron precipitation can be distinguished i.e. stained, coated, and cemented. These precipitation types exert different influences on the stability of manmade slopes as demonstrated by the results of rock discontinuity shear strength analyses using empirical equations, i.e. the inter-block shear strength (Barton et al., 1974) and the sliding criterion (Hack \& Price, 1995). Cemented discontinuities have a larger shear strength than the coated and 
stained discontinuities for the same weathering grade. Analyses of samples using a portable X-ray fluorescence (XRF) instrument for the three types of iron precipitation show that the total iron content for stained, coated, and cemented discontinuities are $2 \%, 5 \%$ and $20 \%$ respectively. The iron content also controls the characteristics and properties of condition of discontinuities (roughness, infill, and resulting shear strength).

The influence of exposure time factor (road excavation time) is related to the deterioration of man-made slopes in time. Slopes along older roads are likely more prone to slope failure compared to slopes along newer roads. This factor has been reclassified into two subclasses; road constructed more than 30 years and road constructed less than 30 years ago. It was used as one of the parameters in the assessment of a Landslide Susceptibility Map (LSM) of the northern Kota Kinabalu area. The result shows that landslides mostly occurred along older roads, thus confirming the hypothesis. However, slope stability or landslides along roads may be also governed by the local discontinuity conditions and the existing mitigation measures applied at some parts of the roads. The current scale of investigation is not be able to capture the contribution of these factors, thus site investigation is essential to verify their stability. The analyses also revealed that the important landslide causal parameters are the anthropogenic and topographical parameters, whereas the least important are the geological and hydrological parameters. The LSM of the northern Kota Kinabalu area is developed based on the combination of anthropogenic, topographical, and hydrological parameters with an AUC value of about 0.8108 . It can be used as basic tool to anticipate and evaluate possible landslide related hazards at the early phase of regional development planning.

In conclusion, the research shows that the deterioration of intact rock properties such as the intact rock strength (IRS), and time relations between weathering and deterioration allow prediction of the reduction of properties at the end of the "serviceable lifetime" of the slopes made in the SST unit. This is likely also applicable to man-made slopes in other humid tropical areas, where similar weathering processes are the main reason for degradation of the rock mass after excavation. The methodology used in this research to establish a weathering-time relation is likely applicable to areas in other climates as well. Furthermore, the research also shows that the effects of weathering processes on rock mass properties can be predicted in terms of an increase in the number of discontinuity sets as well as an increase of the number of discontinuities in each set and the future pattern and spacing of discontinuities from studies of the integral or incipient discontinuities present. The presence or absence of secondary iron minerals also has a marked influence on the properties of the rock mass and discontinuities. Susceptibility to weathering of sandstone with iron-precipitated 
discontinuities is reduced. Therefore, in stability analyses the presences of weathering zones should probably be added to the description of the "condition of discontinuity" in rock mass descriptions and classification systems. 


\section{Samenvatting}

Verwering is een van de hoofdredenen voor instabiliteit in uitgegraven hellingen. Verwering zorgt voor een voortschrijdend verval van het materiaal dat de helling vormt door vermindering van de intacte gesteente sterkte, toenemend aantal breuken, en afnemende schuif- en treksterkte langs en over de breuken. Deze processen worden versterkt door het optreden van druk ontspanning door verwijdering van bovenliggende grond en het uitgraven van de helling.

De gevolgen van verwering en drukontlasting zijn vaak duidelijk zichtbaar langs wegen en andere transport corridors in de vorm van helling instabiliteit voordat het zogenaamde eind van het ontwerp levensduur van de weg is bereikt. Meestal wordt wel onderkent dat de graad van verwering tijdens het maken van de helling in de berekening van de helling stabiliteit voor het ontwerp moet worden opgenomen, echter vaak wordt geen rekening gehouden met verdergaande verwering tijdens de gehele bestaan van de helling. De reden hiervoor is meestal dat er geen of weinig bekend is over hoe de verwering over tijd kwantitatief de gesteente eigenschappen beïnvloed. Daarom is het noodzakelijk dat onderzoek wordt gedaan om relaties te vinden die de vermindering over tijd geven van gesteente massa eigenschappen. Dergelijke relaties kunnen behalve in het ontwerp van hellingen ook worden gebruikt in zogenaamde "landslide susceptibility maps". Dit zijn kaarten die de waarschijnlijkheid van het voorkomen van instabiliteit in hellingen aangeven.

Het onderzoek beschreven in deze thesis betreft het ontwikkelen van kwantitatieve factoren voor het voorspellen van de strekte afname van gesteente eigenschappen over tijd voor de "thick to very thick bedded Sandstone unit" in het tropisch-vochtige klimaat in het Noorden van Kota Kinabalu, Sabah, Malaysia. Karakterisering van de gesteentes is gedaan in uitgebreide en gedetailleerde beschrijvingen van de gesteentes in het veld gecomplementeerd met simpele fysische, mechanische, en chemische tests. De gesteente massa's zijn eerst ingedeeld in units gebaseerd op bedding dikte, lithologie, bedding ratio van verschillende afwisselende lithologieën, en graad van verwering. Graad van verwering is visueel bepaald gebaseerd op BS5930 (1981). Karakteristieken van de gesteentes zijn kwantitatief bepaald per geotechnische unit gebaseerd op de Slope Stability Probability Classification (SSPC) methodologie (Hack et al, 2003). Intact gesteente sterkte is geschat met gebruik van de zogenaamde "simple means" methodologie, welke gebaseerd is op het gebruik van hand en geologische hamer voor schatting van de IRS (BS 5930, 1981, 1999). 
Het ontwikkelen van de kwantitatieve relaties met tijd is gebaseerd op de intacte gesteente sterkte (IRS) per tijdsunit. De gevonden relatie is:

$$
I R S_{t}=105+34 \log (1+t)
$$

$I R S_{t}$ (in $\mathrm{MPa}$ ) is de intacte gesteente sterkte op tijd $(t), t$ is de tijd sinds uitgraven in jaar. De constant 105 is de initiële waarde van de IRS in MPa van de onverweerde SST unit op het tijdstip van uitgraven, en de constant 34 is de zogenaamde "apparent reduction rate" in $\mathrm{MPa} /(\log$ [jaar]). Vergelijkbare relaties kunnen ontwikkeld worden voor andere gesteente massa parameters, zoals cohesie en frictie uit het SSPC systeem. In dit onderzoek zijn verder bestudeerd de ontwikkeling van nieuwe breuken door verwering en hoe die zich verhouden met de al bestaande breuken in het gesteente.

In tropische gebieden speelt chemische verwering een belangrijke rol zoals door de ontwikkeling van oxidatie en oplossingszones in het gesteente. De chemische processen in deze zones kunnen een bijdrage leveren aan de verzwakking van het gesteente door oplossing, echter ook door precipitatie in breuken en intact gesteente een versterking van het gesteente geven. Deze zones hoeven en zijn vaak niet gelijk aan de verwering units. In het ontwerp van hellingen dient hiermee rekenen te worden gehouden.

De invloed van tijd na uitgraving in de toekomstige stabiliteit van hellingen is een belangrijke factor in het maken van "landslide susceptibility maps". Dit is aangetoond door tijdsfactoren te gebruiken in het maken van deze kaarten. Deze laten zien dat veel helling instabiliteit voorkomt langs oudere wegen indien geen onderhoud is gepleegd en dat er een relatie is tussen de hoeveelheid hellingsinstabiliteit en de ouderdom van de hellingen. Dit bevestigd de hypothesis dat tijdsfactoren noodzakelijke zijn en gebruikt dienen te worden in "landslide susceptibility maps".

Concluderend kan worden gesteld dat tijdsafhankelijk verval van gesteente eigenschappen een belangrijke factor is in het ontwerpen van stabiele hellingen. Daarbij moet worden aangetekend dat het simpelweg aannemen dat altijd een verzwakking optreedt niet juist hoeft te zijn, maar dat chemische processen ook een versterking van de gesteente massa kunnen bewerkstelligen. Het onderzoek is gelimiteerd tot tropisch-vochtige condities en tot zandsteen zoals deze voorkomt in Kota Kinabalu. Echter het is waarschijnlijk dat de gevonden resultaten ook toepasbaar zijn op zandstenen in andere tropische regio's, en misschien ook op andere gesteentes. 


\section{Curriculum vitae}

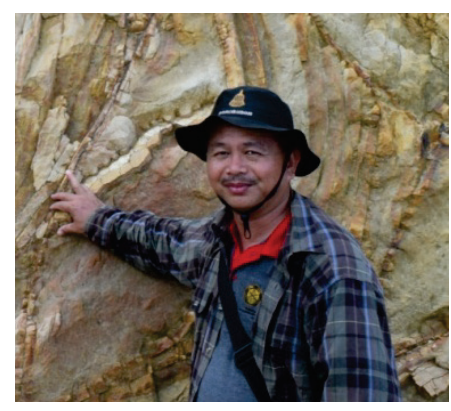

Frederick Francis Tating was born on the 7th of February 1969 in Kampung Papar Tambunan, Sabah Malaysia. He started his early education (primary level) at St. Theresa Primary School in Tambunan in 1975 and continued his secondary education at St. Martin Secondary School, also in Tambunan until 1983. He continued his secondary education at the first Sabah Boarding School (Sabah Fully Residential School) in Kota Kinabalu, Sabah until 1985 and Pre-university (Matriculation Class) at the same school in 1986. He did his tertiary education at National University of Malaysia (UKM), with a major in Earth Science in 1987 and graduated in 1991. After graduation, he joined the Minerals and Geoscience Department of Malaysia (formerly Geological Survey of Malaysia) as a temporary junior geologist and confirmed to the post in 1994. He was assigned to carry out hydrogeology and engineering geology investigations throughout the state of Sabah.

In 2001, he was awarded a Japanese government scholarship (MONBUSHO) to pursue a Master Degree study in Kumamoto, University Japan and successfully completed this study in 2003. His thesis is regarding the relationship between landslide incidents and rock types in Kota Kinabalu. From 2003 to 2008, he undertook several geological projects such as geological terrain mapping, landslide investigations, and reviewing geological and geotechnical reports pertaining various infrastructure development. In 2008, he was awarded a Malaysian government scholarship to pursue a PhD study at Faculty of Geo-information Science and Earth Observation (ITC), University of Twente in the Netherlands. His research focussed on the degradation of sandstone in tropical areas and the study was carried out at Kota Kinabalu Sabah. The outcome of the research was documented in this thesis. At present, he is still working in the Minerals and Geoscience Department of Malaysia as a principal geologist dealing with engineering and environmental geology. 


\section{Publication related to this research}

Tating, F.F., Hack, H.R.G.K. \& Jetten, V., 2015. Influence of weatheringinduced iron precipitation on properties of sandstone in a tropical environment. Quarterly Journal of Engineering Geology and Hydrogeology. ISSN: 1470-9236. p. 17. (submitted and accepted for publication pending modifications)

Tating, F., Hack, R. \& Jetten, V., 2015. Weathering effects on discontinuity properties in sandstone in a tropical environment: case study at Kota Kinabalu, Sabah Malaysia. Bulletin of Engineering Geology and the Environment. 74 (2). DOI: 10.1007/s10064-014-0625-5. ISSN: 1435-9529. pp. 427-441.

Tating, F.F., Hack, H.R.G.K. \& Jetten, V., 2015. Landslide susceptibility assessment using information value statistical method: a case study at northern Kota Kinabalu, Sabah Malaysia. The Malaysian Journal of Remote sensing and GIS. Vol. 4 (2). p. 18.

Tating, F.F., Hack, H.R.G.K. \& Jetten, V., 2014. Quantification of weathering process in thick bedded sandstone of the Crocker Formation in Kota Kinabalu, Sabah. In: Minerals and Geoscience Conference, Hotel Aseania, Langkawi Island, Malaysia, 17-20 June 2014. Minerals and Geoscience Department, Malaysia. 159, pp. 21-28.

Tating, F.F., Hack, H.R.G.K. \& Jetten, V., 2013. Engineering aspects and time effects of rapid deterioration of sandstone in the tropical environment of Sabah, Malaysia. Engineering Geology. 159. DOI: 10.1016/j.enggeo.2013.03.009. ISSN: 0013-7952. pp. 20-30.

Tating, F.F., Hack, H.R.G.K. \& Jetten, V., 2013. Weathering and deterioration as quantitative factors in slope design in humid tropical areas; Case study Northern Kota Kinabalu, Sabah, Malaysia. Ingeokring Newsletter. 33. ISSN: 1384-1351. pp. 22-28.

Tating, F.F., Hack, H.R.G.K. \& Jetten, V., 2013. Quantification of rock mass deterioration process for cut slope design in humid tropical areas; Case study Northern Kota Kinabalu, Sabah Malaysia. In: Taib, N.I. (Ed.) National Geoscience Conference 2013 of the Geological Society of Malaysia (NGC2013); Geoscience for environmental wellbeing, Ipoh, Malaysia, 8-9 June 2013. Geological Society of Malaysia, Kuala Lumpur, Malaysia. paper B24, pp. 102-104. 


\section{ITC Dissertation List}

http://www.itc.nl/research/phd/phd graduates.aspx 